\title{
Avaliação automática de Exames de Proficiência em Inglês
}

\author{
Valéria Tomas de Aquino
}

\author{
Orientadora:
}

\section{Profa. Dra. Sandra Maria Aluisio}

\begin{abstract}
Dissertação apresentada ao Institulo de Ciências Matematicas e de Computação ICMC-USP. como parte dos requisitos para obtenção do tilulo de Mestre em Ciências de Computação e Matemática Computacional.
\end{abstract}

\author{
USP - São Carlos \\ Novembro/2001
}

Data da Defesa:

$14 / 11 / 2001$

Visto do Orientador fand thane ficus. 
As boncäos que Dens tem proporrionado em minha vida. aos mens querido pais pelo amor incondicional. aos amigos que acreditaram em mim. 


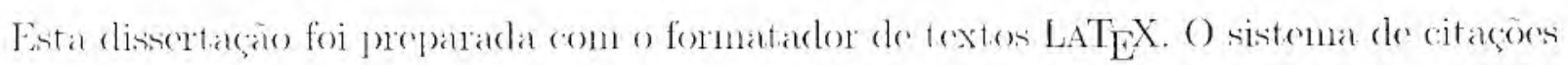

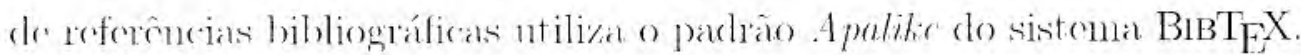

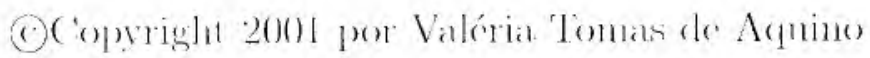

Torlos os Direitos Reservados 


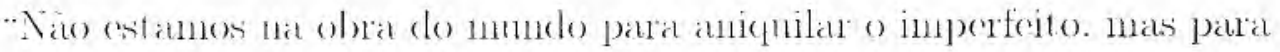

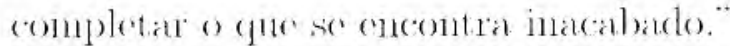




\section{Agradecimentos}

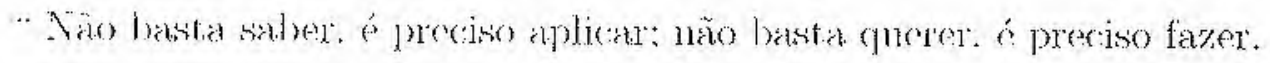
(Gionthe. 1749-1832)."

If

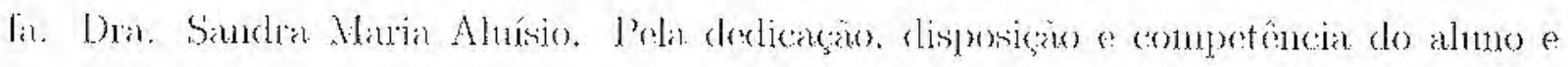

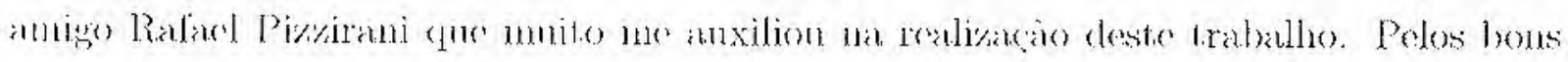

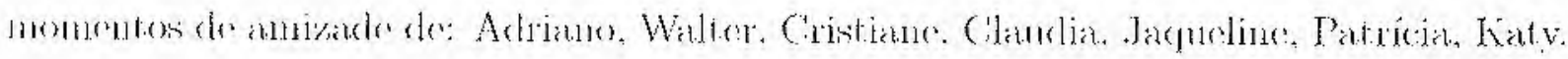

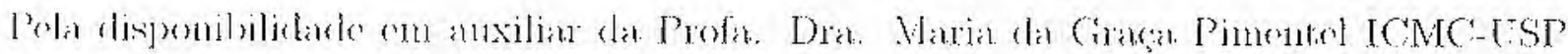

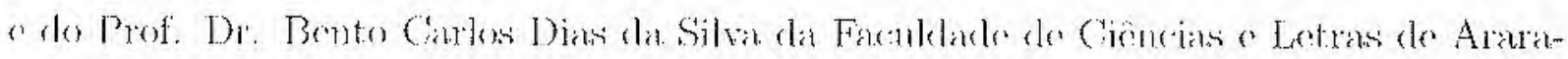

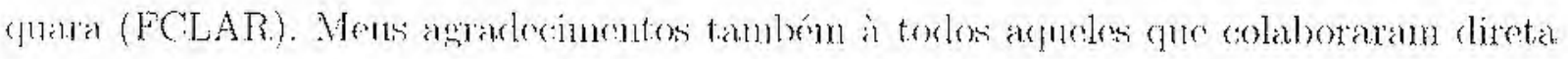

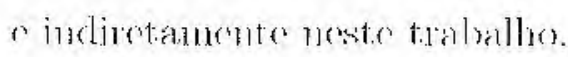




\section{Resumo}

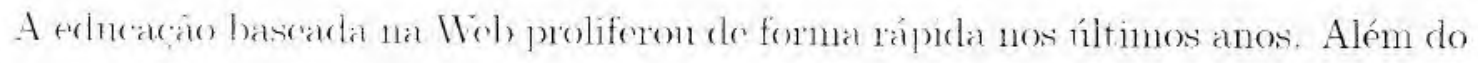

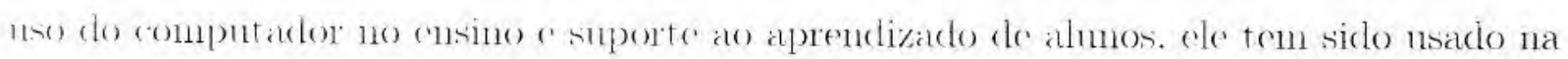
construçăo e aduninistração de testes. A participacăo do computador 110 processo de avaliaçäo pode se dar através de métodos convencionais informatizados on métodos alternativos. A principal característica dos métodos convencionais ó classificar se o conlecimento

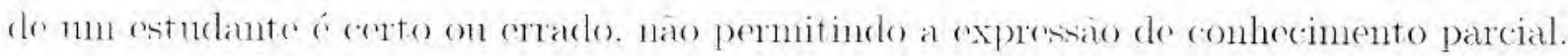

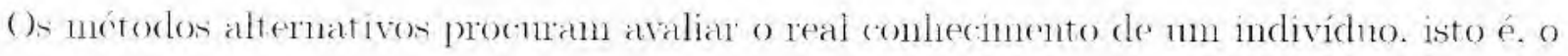
(puanto ele sabe e mulitats vezes sao bascados em teorias estatísticas bastante elaboradas que levam enu conta o fator de adivinhagäo (chute). o nível de dificuldade e o índice de

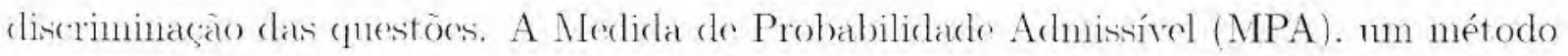
alternativo mais simples. (onsiste na tentativa de quantifican o conherimento parcial de 1311 estudante pon meio de associaçoes muméricas ponderadas a cacla opção de resposta

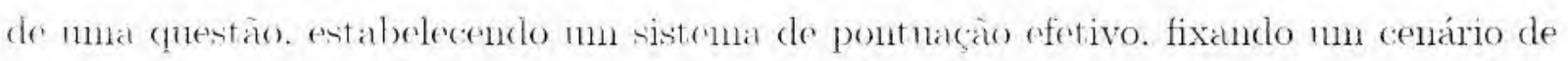
recompensas o penalidades associadiss a cada alternativa. Lina ontra (puestäo relacionada

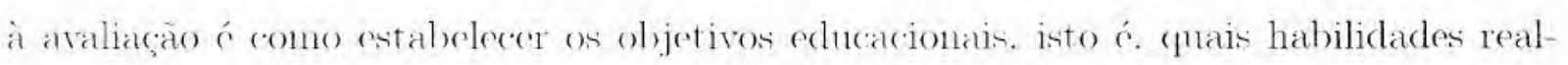

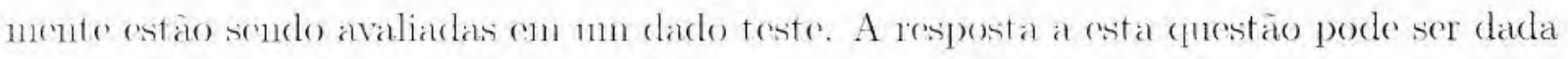
pela Taxomomia de Objetivos Erlucarcionais de Bloome. Este projete de mestrado propôs

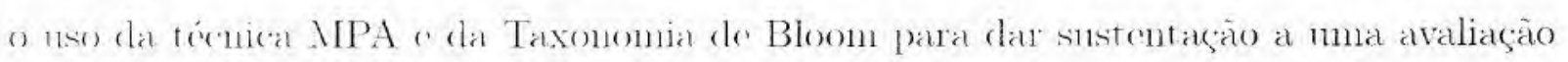
informatizada " de mollow qualidade no crnário do exanue do proficiencia em inglés paJa crusos de mestrado. Un sistema de avaliação haseado na Web. chamado CAPTEAP (Computer Assisted Proficiency Test of English for Academic Purposes). que ntiliza telas

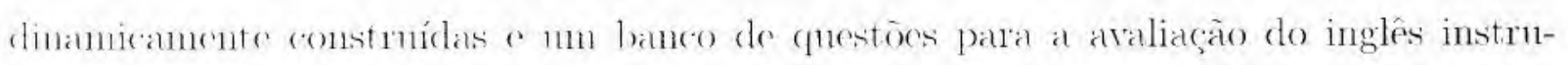

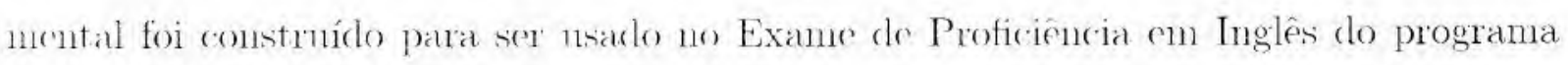
de nestrado do ICMC-LSP. Cun sistena construído nester moldes podo ser aplicado em

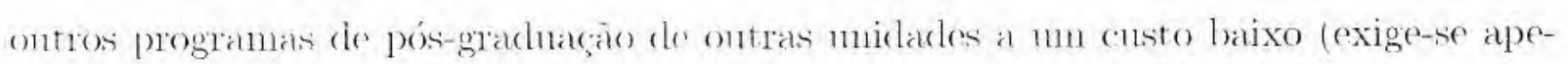
uats una sala com microcomputadoren individuais) "se acrescido cla avaliaçào de ontras labbiliclades pode tambóm ser aplicato a programas do domtorado. 


\section{Abstract}

Over the hast yoars. Web-based education has bernextended to develop and administer tests. The assessment process by computers in conventional methods classify the stmelent knowledge as right or wrong and nusually does not allow for evaluation of partial knowleclge. Alternative nethods try to cvaluate the real knowledge of a student and are generally based on enmplex stat istical theories nsing sereral paraneters such as gnessing. ferel of difficulty and discrimination power of a question. Admissible Probability Measure

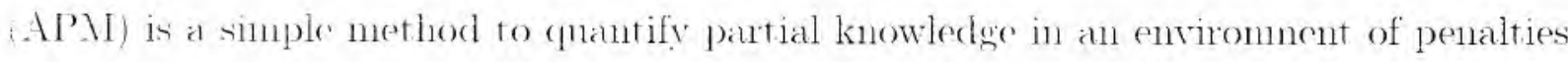
and rewards. Another inportant issise related to assessinent is to determine which abilities are being measured in a test. The Bloom 's Taxomony of Educational Objectives an provide an answer to this question. This project has made use of APM and Bloom s Taxomomy to develop a computer assisted English Proficioncy Tests targeted at MSc. stmelents. The web-based srstem is named CAPTEAP (Computer Assisted Proficiency Test of English for Academie. Purposes). and uses dynamie pages and a database of ins1rmuental English questions. The system hats been applied in proficiency tests for the ISTe progran at ICMC-ISP. Althongh built for this particular program the system is sullieinenty generie to be used in oflece master prograns and may be extended also for PhD programs. 


\section{Sumário}

1 Introdução 1

2 Avaliação Acadêmica - Aspectos Gerais 6

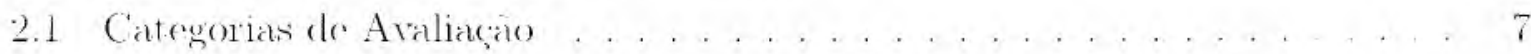

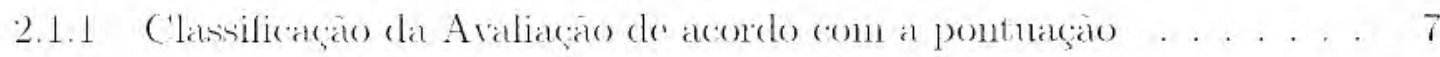

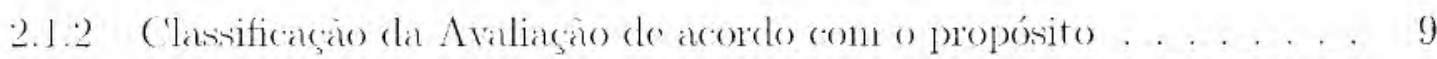

3 Testes Objetivos Informatizados 14

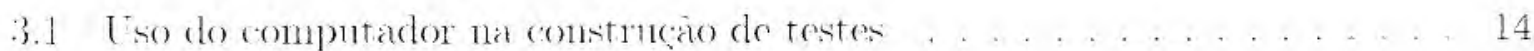

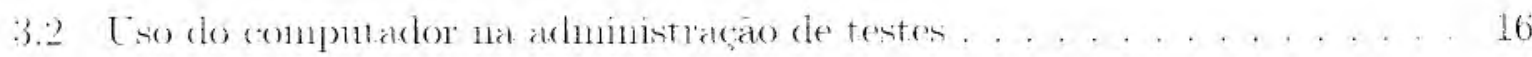

3.2 .1 Caracteristicas do teste . . . . . . . . . . . . . 17

3.2 .2 Inplementacaro do teste . . . . . . . . . . . . . 20

3.3 Estruturat das (Questoes . . . . . . . . . . . . . . . . . 25

3.1 Tipes de fluestions . . . . . . . . . . . . . . . . . . . . . . 26

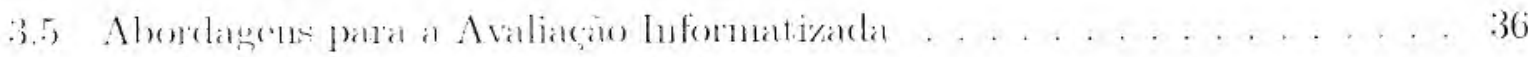

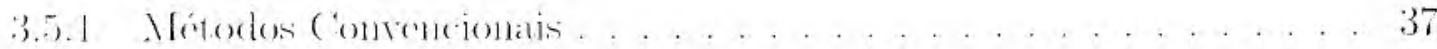

3.5 .2 . Yétodos Altematives. . . . . . . . . . . . . . . . . . . . 38

4 Taxonomia de Objetivos Educacionais de Bloom 45

5 Testes bascados na Medida de Probabilidade Admissível (MPA) 56

5.1 Crianaro do método MPA . . . . . . . . . . . . . . . . . . 56

5.2 MPA adaptada por Bruno (1986). (1987). (1988) . . . . . . . 62

5.2.1 MPA con escala modificada-Confidenere Weighted-Adnissible Pro-

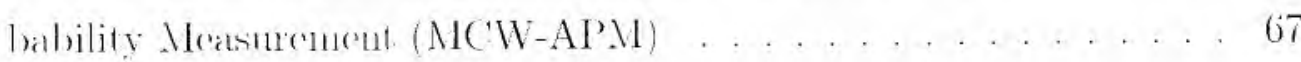




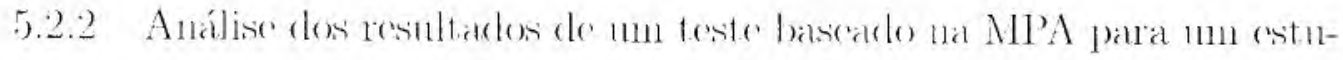
dante considerade frateo . . . . . . . . . . . . . . . . 68

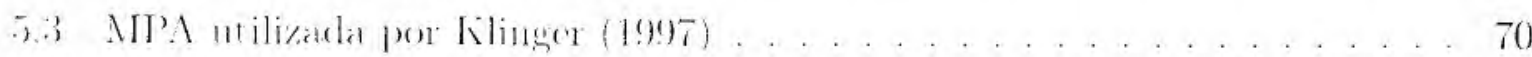

6 Avaliação dos Exames de Proficiência em Inglês Desenvolvidos $\quad 72$

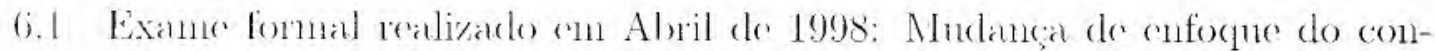

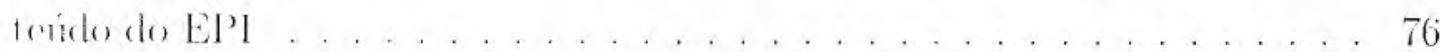

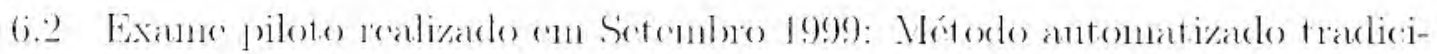

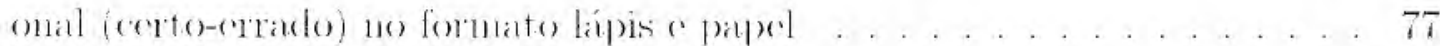

6.3 Exame formal realizato em Setombo 2000) MPA uo formato lápis o papel so

6.1 Exame piloto realizado sm Fovereiro 200)1: Exame Online . . . . . . . . . 82

6.5 Exame formal realizado (an Abril 2001: М.PA no Fxanne oficial online . . 84

6.6 Avaliacão da Tarabilidade do Exame realizado em Abril de 2001 . . . . . 92

6.6 .1 Planejanento . . . . . . . . . . . . . . . . . . . . . . .

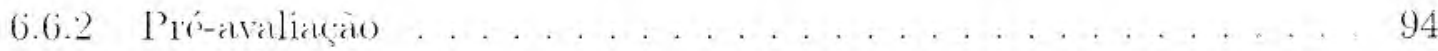

6.6 .3 Avaliatas . . . . . . . . . . . . . . . . 94

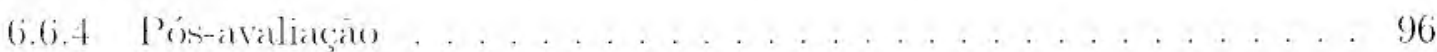

7 Um sistema baseado na Web para o EPI para o mestrado do ICMC-USP106

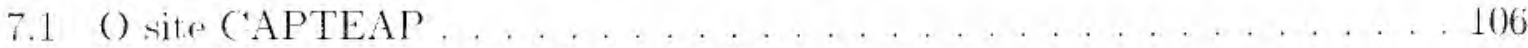

7.2 Arepuitertura do sistenta . . . . . . . . . . . . . . . . . 114

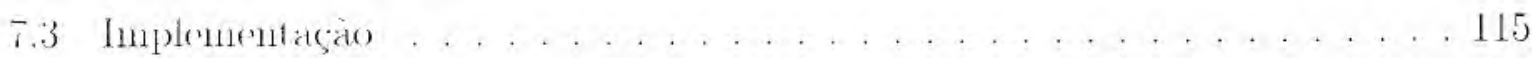

8 Conclusão 121

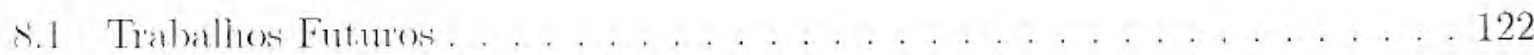

Referências Bibliográficas $\quad 124$

$\begin{array}{ll}\text { A Exame formal realizado em Abril de } 1998 & 127\end{array}$

B Exame piloto realizado em Setembro de 1999

C Exame formal realizado em setembro de 2000 
D Exame piloto realizado en Fevereiro de 2001

E Exame formal realizado em Abril de 2001 


\section{Lista de Figuras}

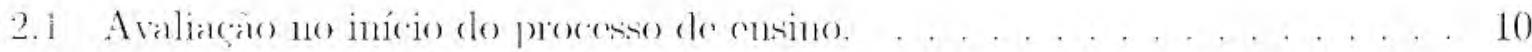

2.2 Avaliagat Fomutiva . . . . . . . . . . . . . . . . . 10

2.3 Avaliacaio Final. . . . . . . . . . . . . . . . . . . . . . . 11

2.4 Avalialçoo Contintada . . . . . . . . . . . . . . . . 12

3.1 Template para as questoos solne triângules (Alessid and Trollip. 1991). . . 15

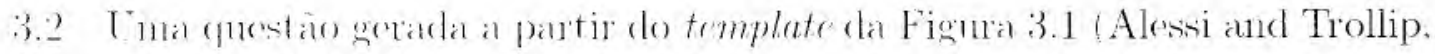
19911.

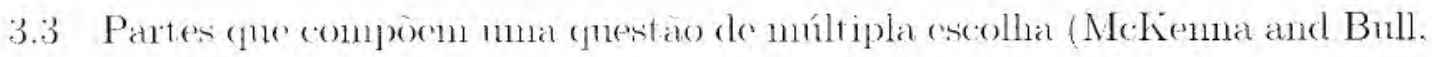
1999).

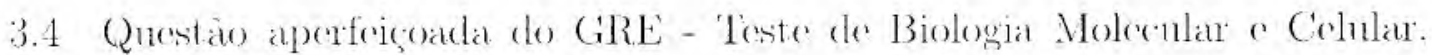
1997-1999) (Mckinnat and Bull. 19999).

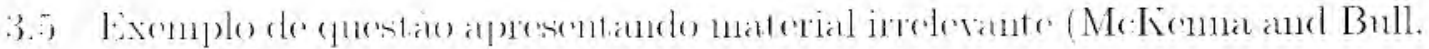
$19999)$

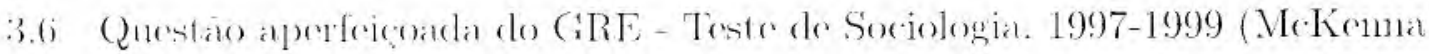
ancl Bull. 19999).

3.7 Questäo aperfeicoarla do GRE - Teste do Sorciologia, 1997-1999 (Mc.Kenna and Bill. 19999).

3.8 (Questoes do live de teste GRE Sociologia. 1997-1999 (MeKema and Bull. $1999)$

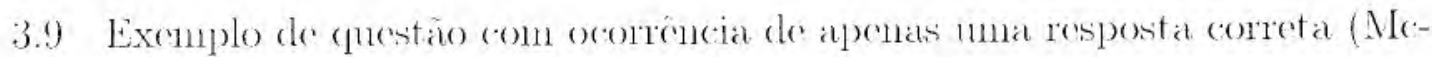
lien1ta and Bull. 1999) . . . . . . . . . . . . . . . . . 32

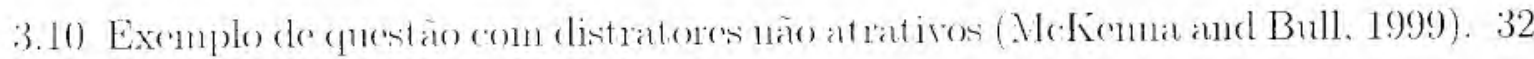




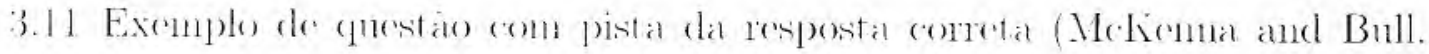
$19999)$.

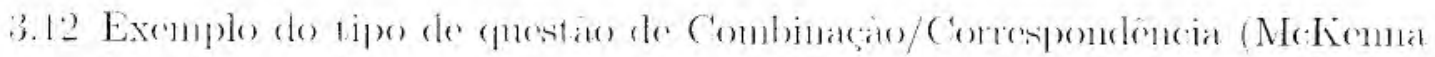

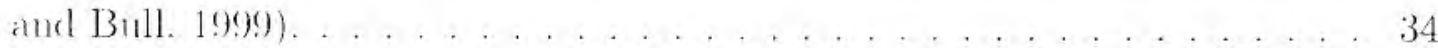

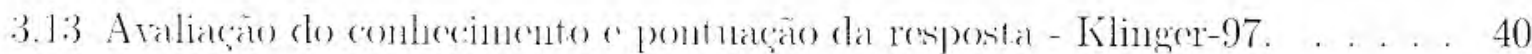

3.14 Serão o estrutura do GRE CAT . . . . . . . . . . . . . 43

3.15) Estabolerendo a ponthacão no GRE CAT . . . . . . . . . . . . 44

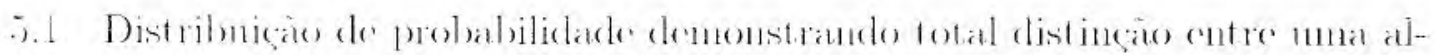

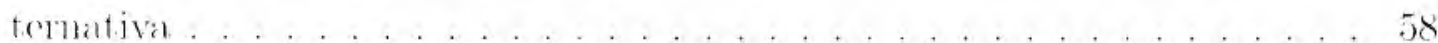

5.2 Distribuicão de probabilidade quando năo se distingue entue duas alternativas 58

5.3 Distribuicão de probabilidade guando não se distingue entre tress alternativas 59

5.4 Trmos utilizados para caracterizar as regiones dos espacos de probabilidades (Shmerol d Brown. 1974).

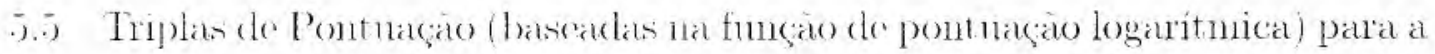

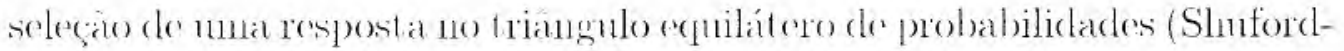
74).

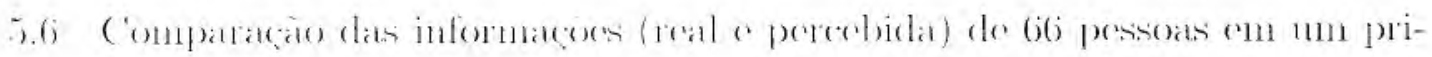

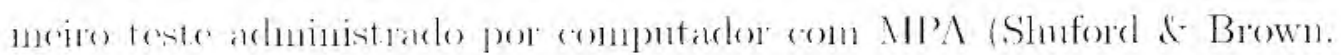
1974

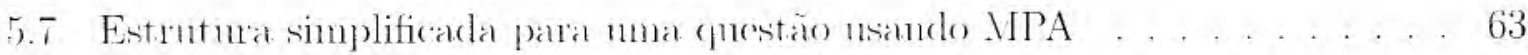

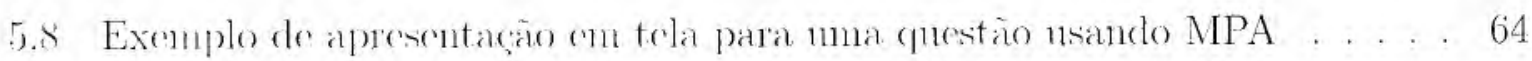

5.9) Análise de restultados - Brnuo-86 . . . . . . . . . . . . . . 65

5.10 Análise de resultados - Brnuro-\&6 . . . . . . . . . . . . . . . 66

5.11 Análise de resultados - Klinger-97 . . . . . . . . . . . . . . 71

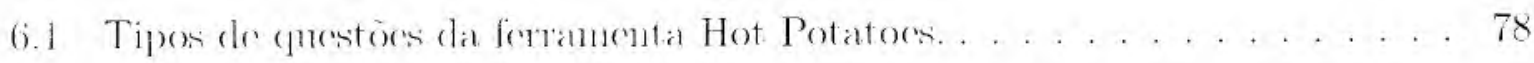

(6.2 Apresentación dos resultados inclividuais do rxame do Setembro de 2000 . . 81

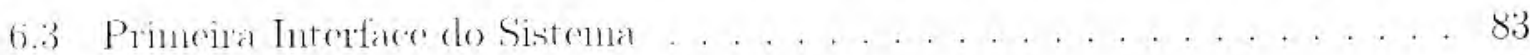

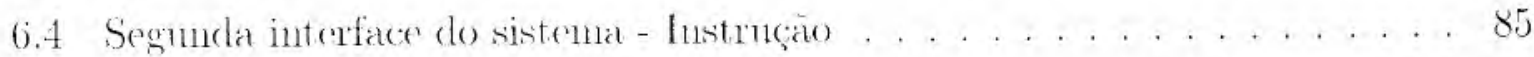

6.5 Segunda interface do sistenla - Explicaçao . . . . . . . . . . 85

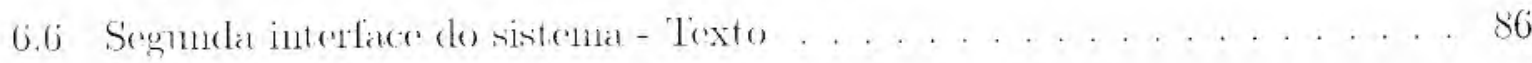




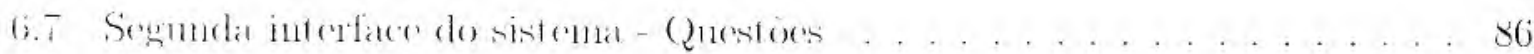

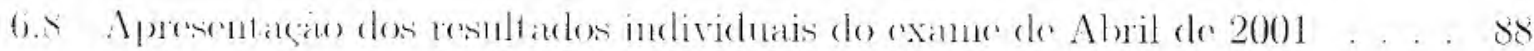

(j.9) Interfaner de Cadastro. . . . . . . . . . . . . . . . . . . . . . . . . .

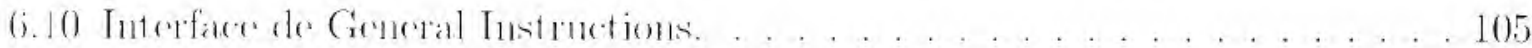

(i.]) Interface de Contonts. . . . . . . . . . . . . . . . . 105

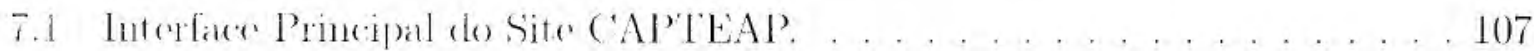

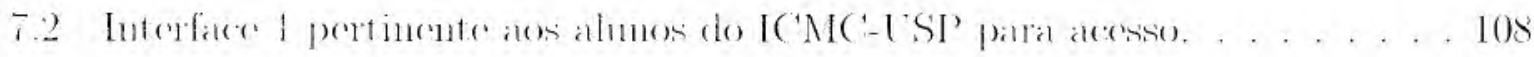

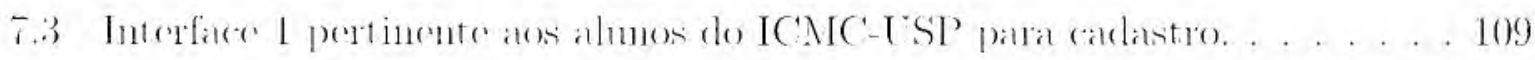

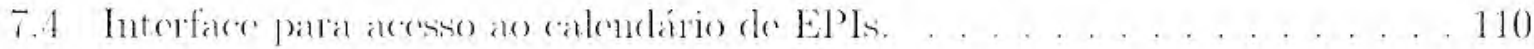

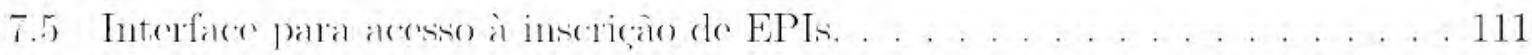

7.6 Interface para acesiso alo galbarito de EPIs. . . . . . . . . . . . . 112

i. Interface para acesso ans resultados de EPIs. . . . . . . . . . . . 113

T.8 Interface para alecsiso a pratica de EPIs. . . . . . . . . . . . . . 114

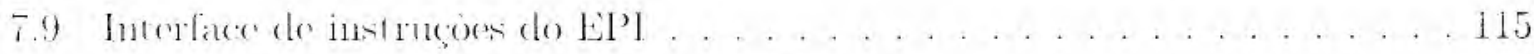

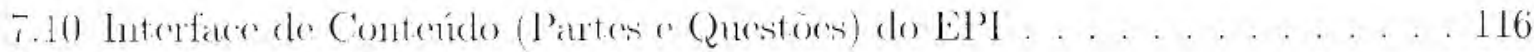

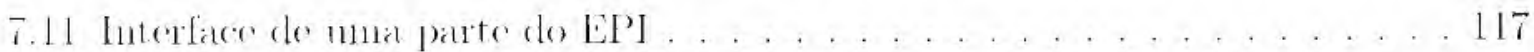

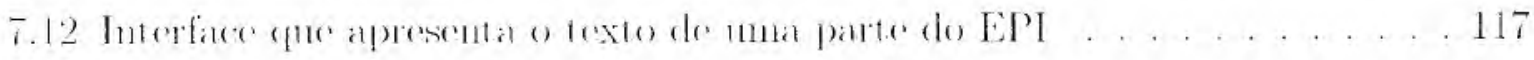

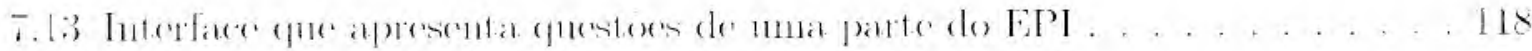

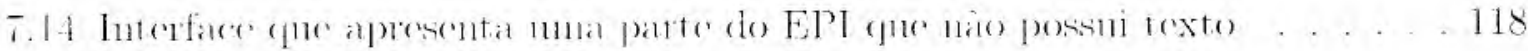

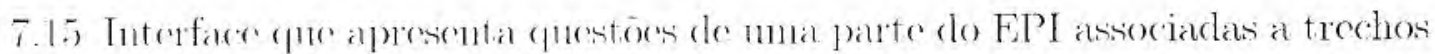
detextos . . . . . . . . . . . . . . . . . . . . . . 119

7.16 Interfacen do priblico geral . . . . . . . . . . . . . . . . . . . . . 119

7.17 Diagrana de blocos geral dos principais módulos do El’ . . . . . . . 120

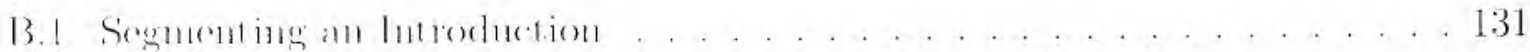

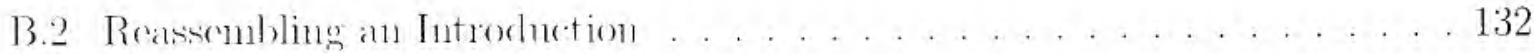

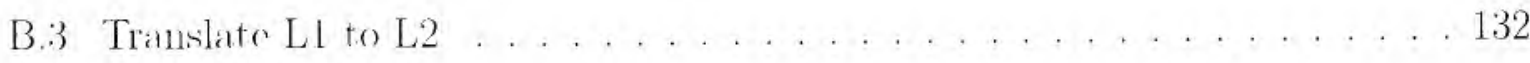

B.4 Translate L2 to L1 . . . . . . . . . . . . . . . . 133

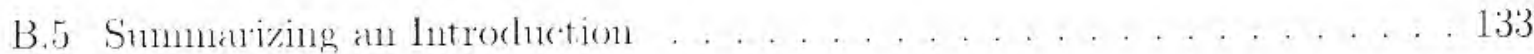

B.6 Strategies to write Stages . . . . . . . . . . . . . . . . . . . . . . .

13.7 Roformulating Text . . . . . . . . . . . . . . . . 134 


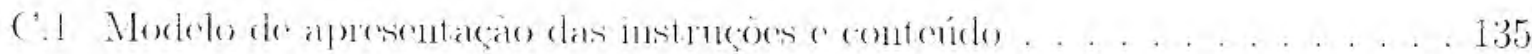

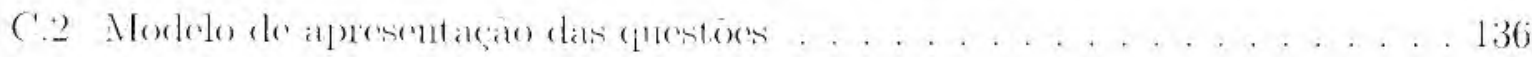

D. I Modelo de apresentacaio das questoes . . . . . . . . . . . . 146

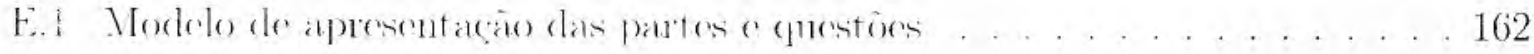




\section{Lista de Tabelas}

3.1 Exemplo do tipo de (puestao de Completar Múltiplo (Miller-98). . . . . . 33

3.2 Exemplo de (puestào do tipo Análise de Redacăo/Cansa-Efeito (Miller-98). . 35

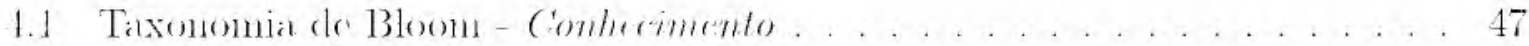

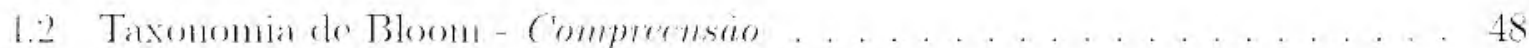

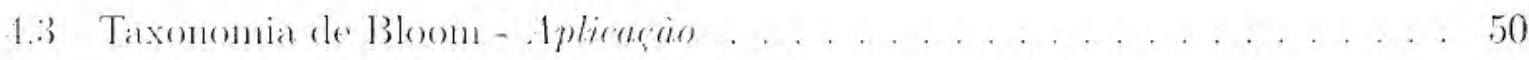

4.4 Taxonomia de Bloom - Análise . . . . . . . . . . . . . . . . 51

4.5 Taxonomia de Bloom - Síntese . . . . . . . . . . . . . . . 52

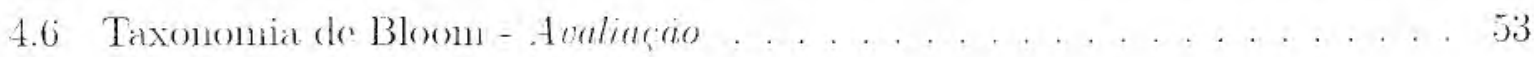

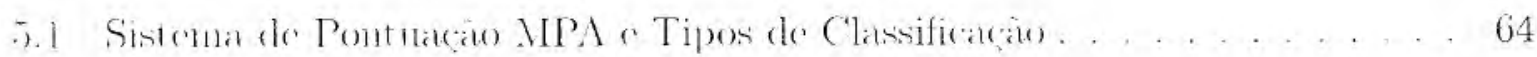

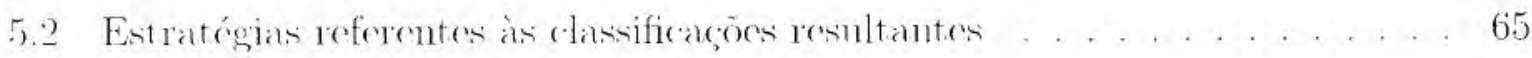

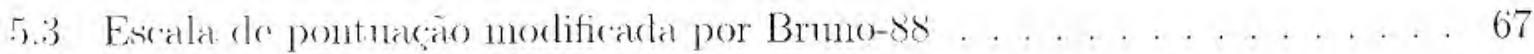

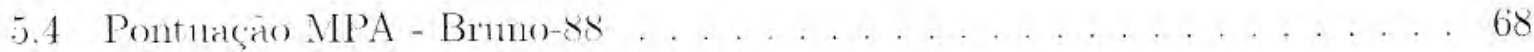

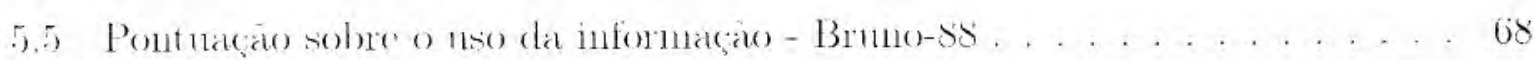

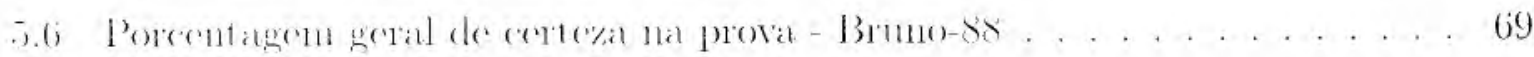

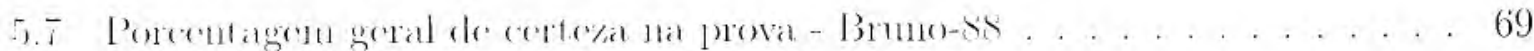

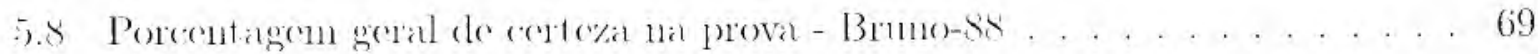

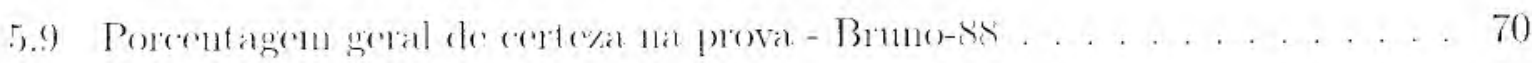

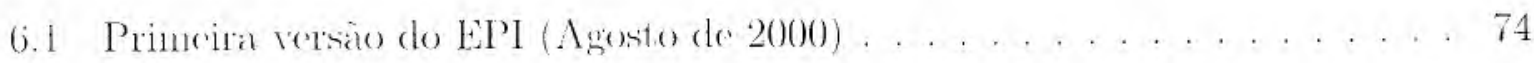

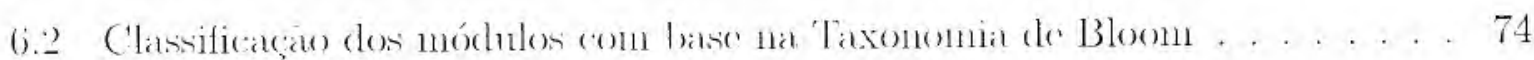

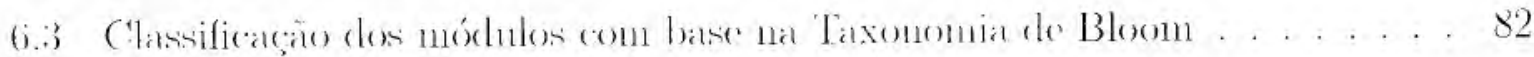

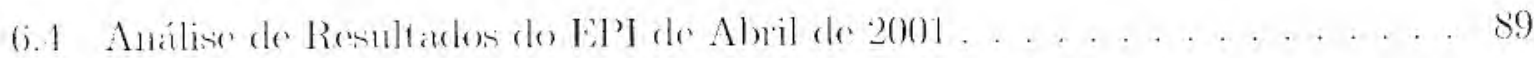

(6.5) Versaio atnal do conterído do EPT . . . . . . . . . . . . . . . . 91 


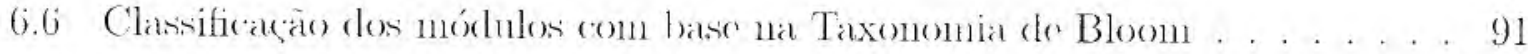

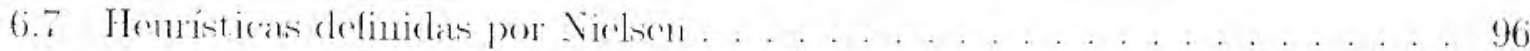

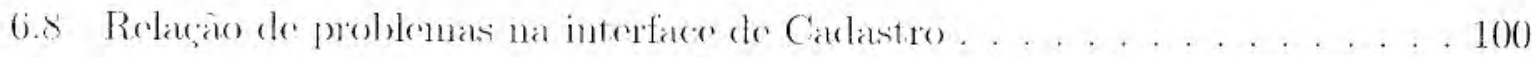

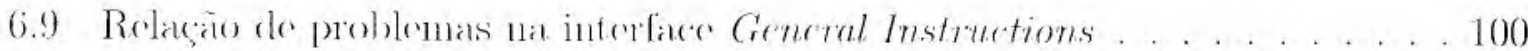

6.10 Rerlacãos de problemas na interfanes Contents . . . . . . . . . . . . 101

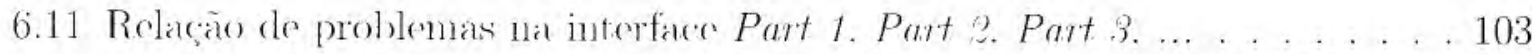

6.12 Relacão de problemas na interface Resultados . . . . . . . . . . . . 104 


\section{Capítulo 1}

\section{Introdução}

Inscrialat 110 contexto da arte de ensinar. a avaliaçào assime importantes e definitivos papéis. Dependendo do propósito. a avaliação pode nectir a qualidade on o potencial

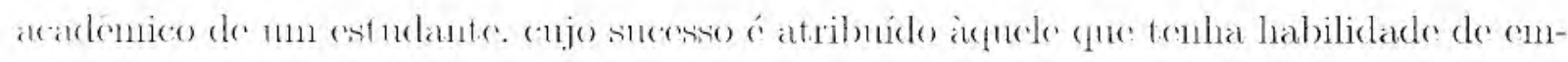

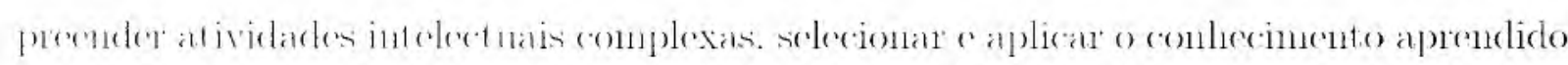

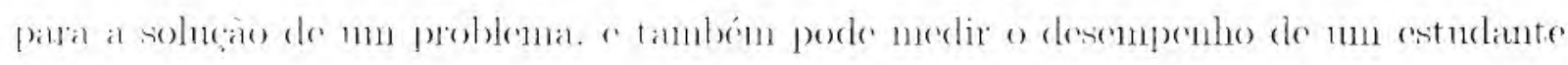

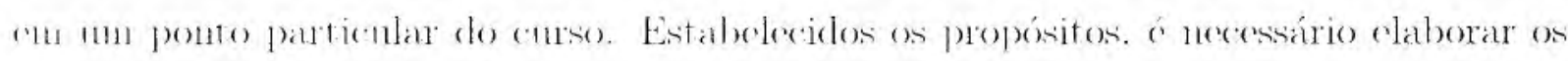
testers/exames que possibilitem atingi-los.

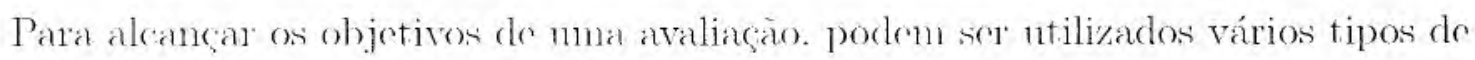
restes (Miller et al. 1998). Testes de avaliação djagnóstica. por exemplo. tôm a função do insercäo do estudanites en programas do ensino adequados. São empregados antes do

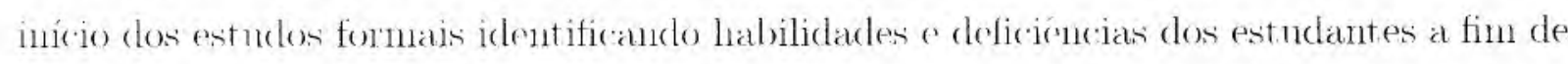

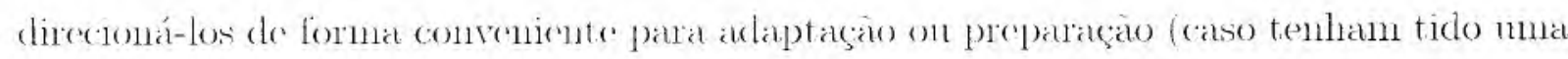

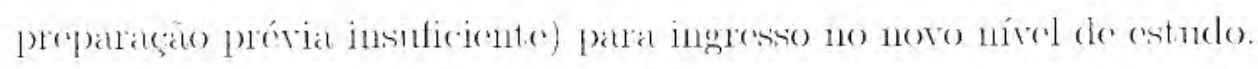

Exrréreios. trabathose testes progressivos dados ano estudante ao longo do ano on

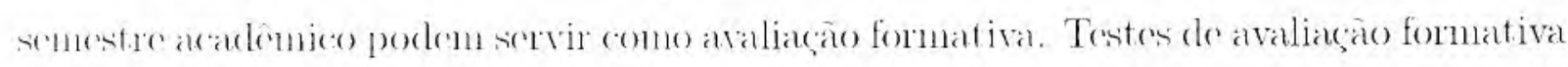

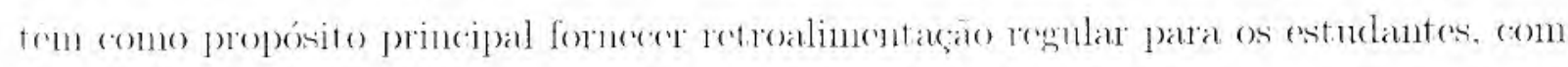

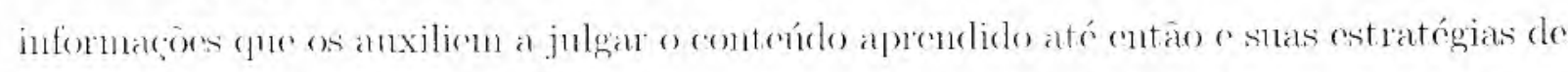

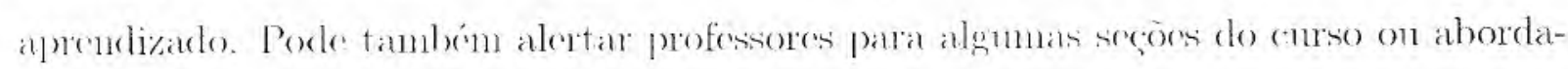
gens de ensino ('nu gue os estuddantes estrejam tendo dificuldades, e. portanto, para as quais será neressírio maior atenção. Já no asso de num teste de avaliação final. o propósito prin- 


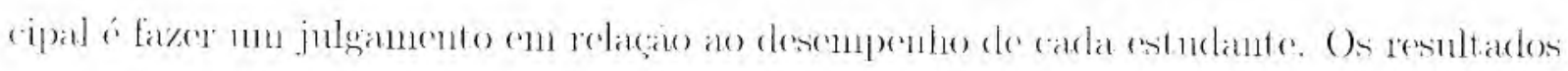

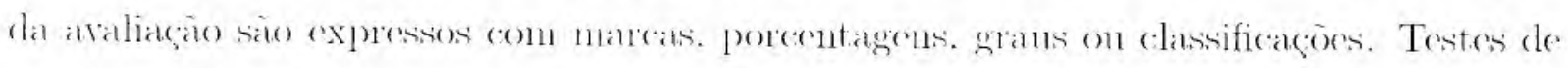

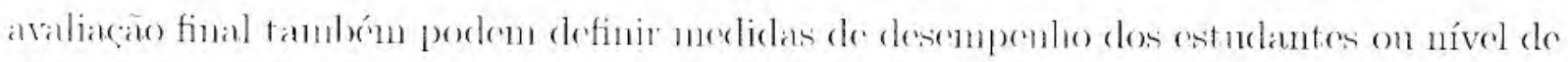

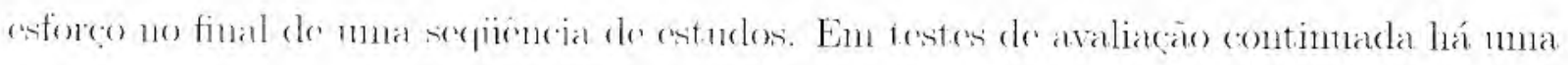

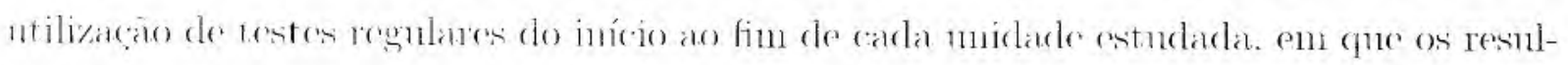
tados de cacta passos do traballo contribuem para o resultado final. Testes de avaliacão

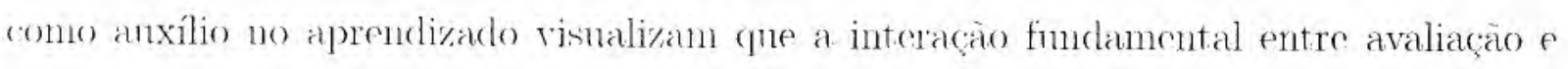
aprendizado ocorre quando existe mua mistma entre tarofas que sào cladas ao estudante. testanclo seris progressos on esforecos e objetivos de proganats de enisino.

() compratador pode participar da construçio o administraçà dos testes. porém.

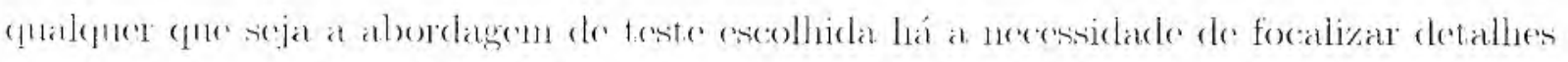

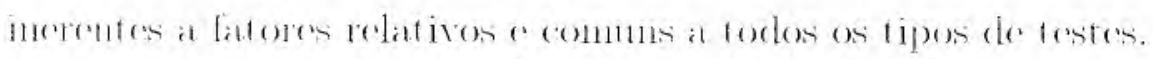

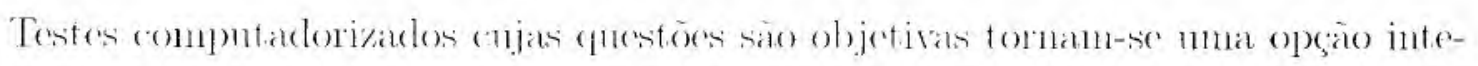

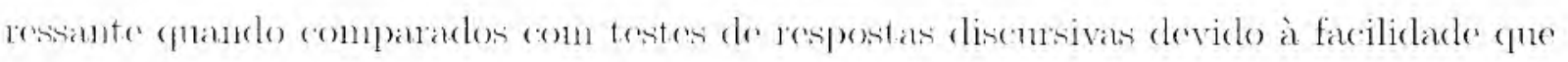

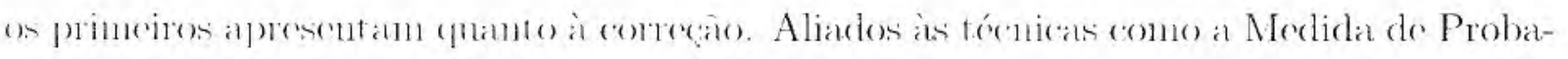
bilirlarde Admissírel (MPA) (Shuford d. Brown (1974). Brmo (1986). Klinger (1997)) que

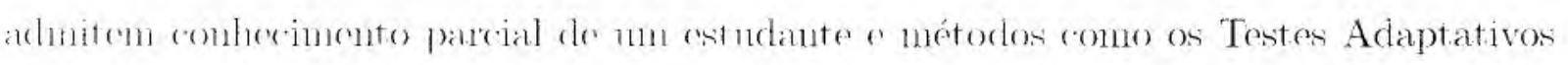

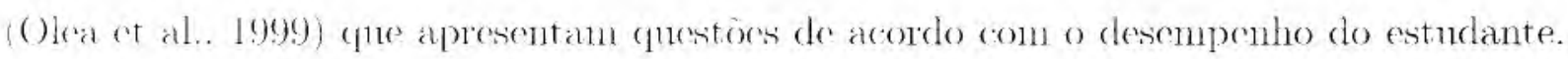
os testes possibilitam estabelecer avaliacoes efetivas das proficiencias dos estudantes.

O) dominio escolledo para este mestrado foi o exame de proficiencia em inglês

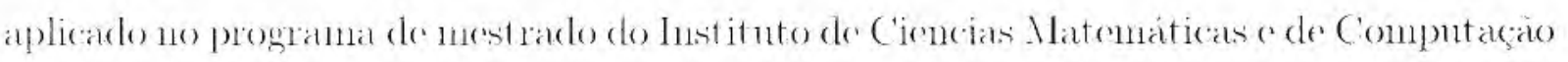

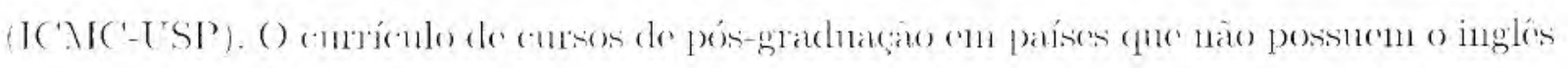

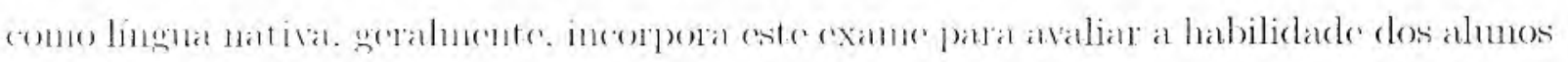

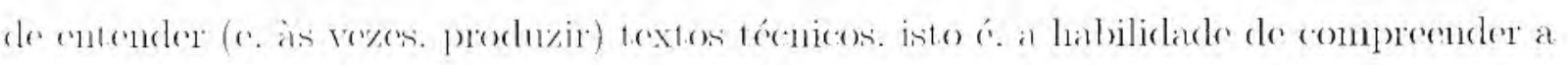

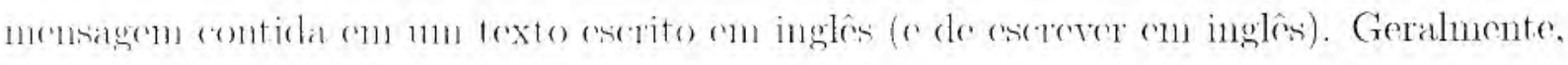

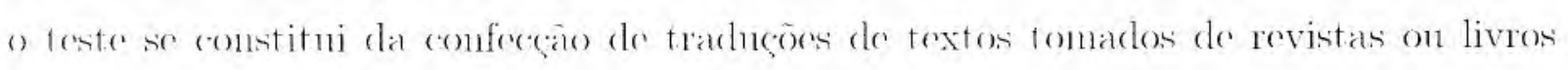
récuicos escritos on inglos (T.2) paya a língua nativa (portuguess, por exemplo) (L1). e. cun casos raros. da producào da versaio an inglês de ontro texto de literatura técnica. originatiannente. 1ha língua nativa. Até o início de 2000) os estudantes que ingressavam no

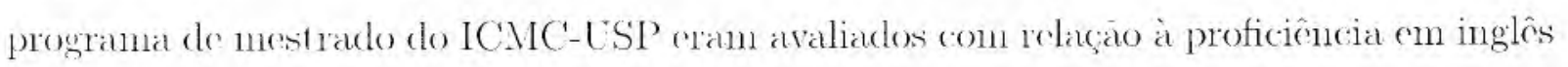




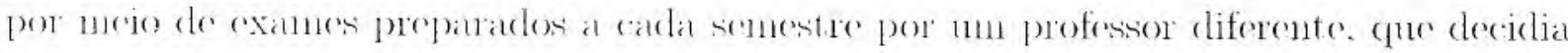

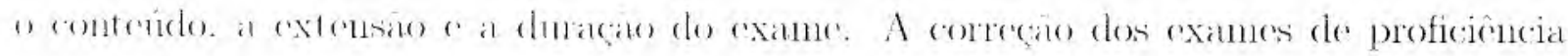

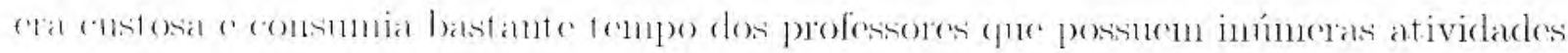

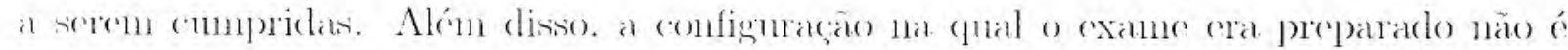
irleal. pois exames distiutos podem ser fortenente näe miformes devide à rotatividade do professores responsáreis. o também à subjetividade inerente à construção e correção lumanas. Exannes que reguerem somente traducoes de l2 para L1. e vice-versa. são anda mais problemaiticos e linitantes. pois só avalianu numa competencia do estndante: a comprecusato do texto. A "conscioncia do genfero". isto a a consciencia da estrutura e

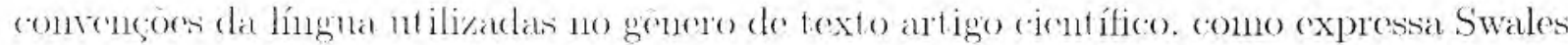

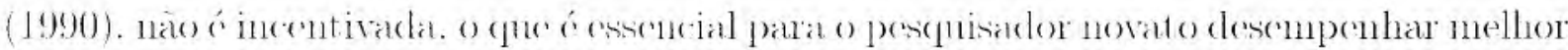

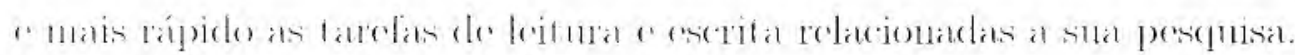

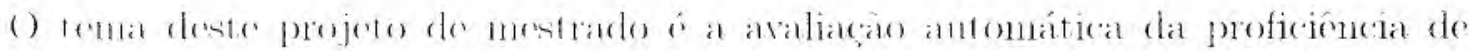

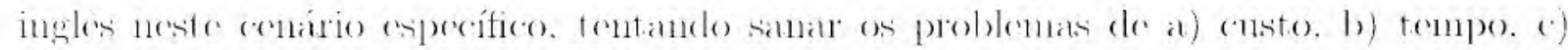

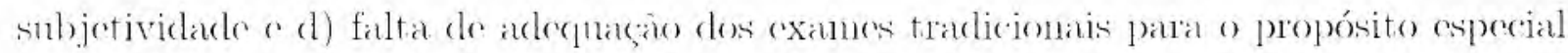
de se avaliar mon estudaute que deve possinir 1110 bom desompenho na leitura do ingless

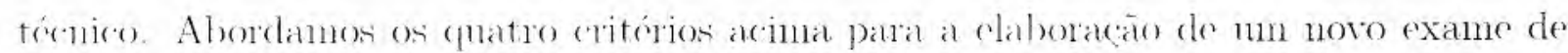

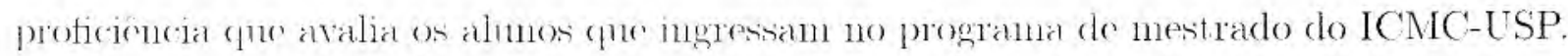

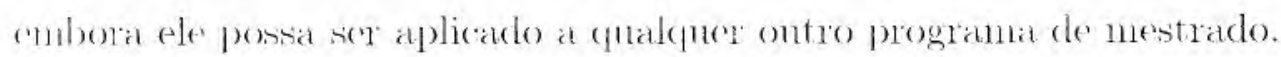

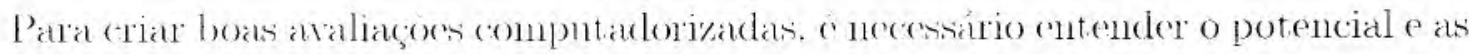

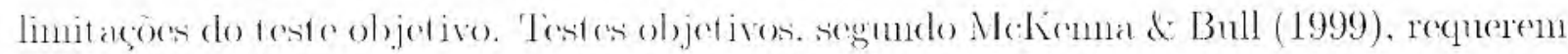

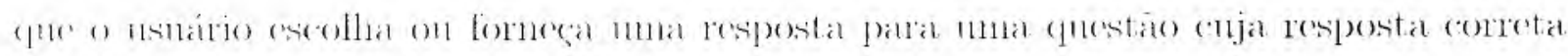

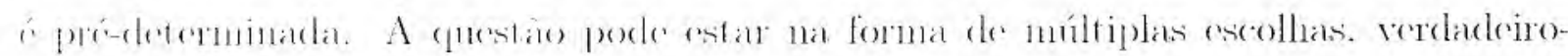

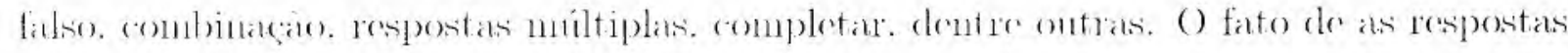

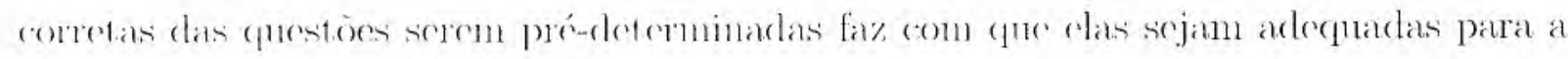

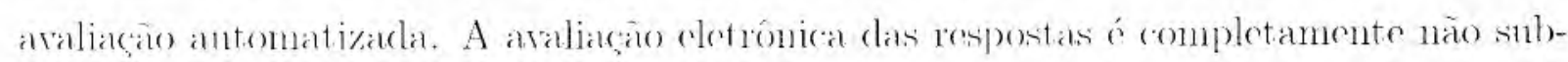

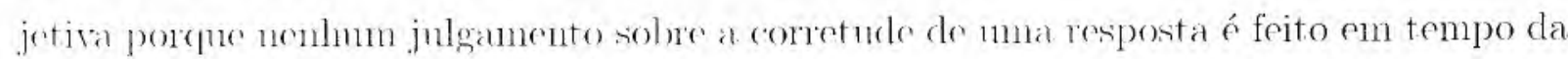
avaliacăo. As vantagens de testes objotivos são as segunutes: permitem professores avalia-

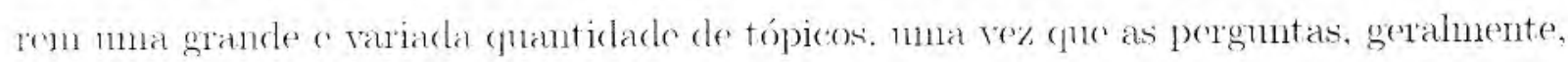

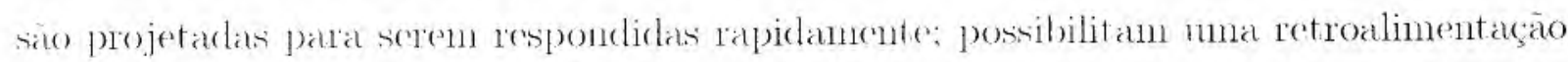




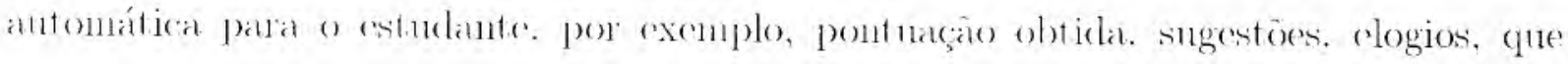

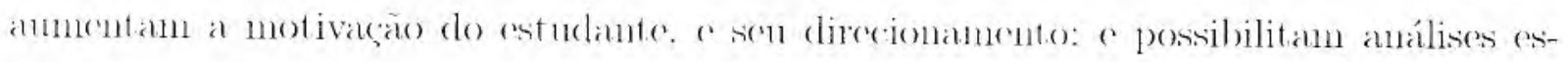

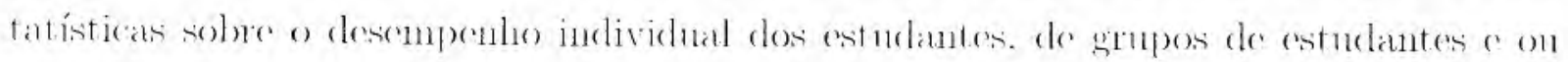
pergintats.

Darta a experiencia da orientadora deste projete de mestrado na construçăo de

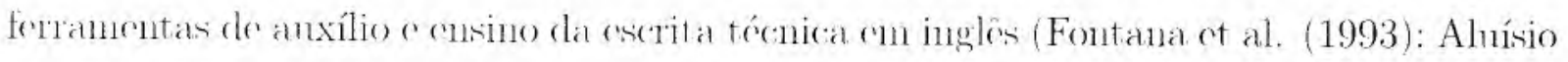
\& Oliveira (1995): Aluísio \& ()liveira (1996); Alú́sio \& Gantenbein (1997): Aluísio et al.

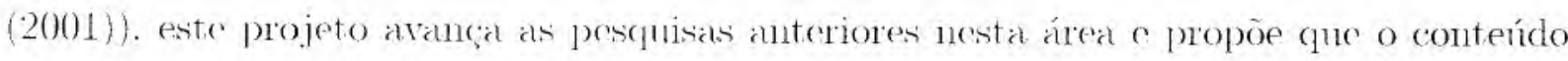
dos examen de proficioncia rm ingles para adnissaco no mestrado do ICMC-USP seja

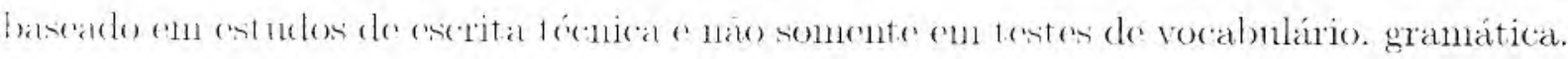

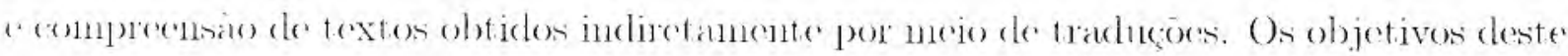

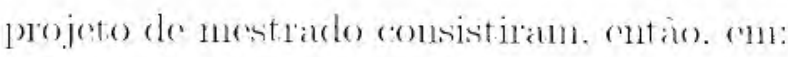

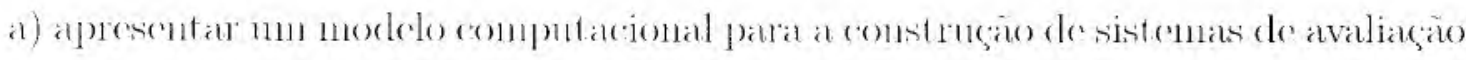

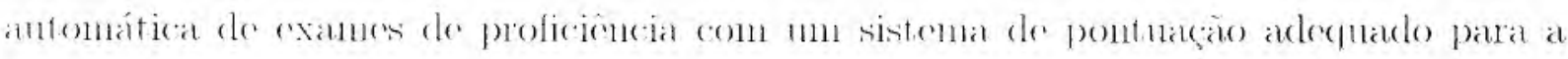

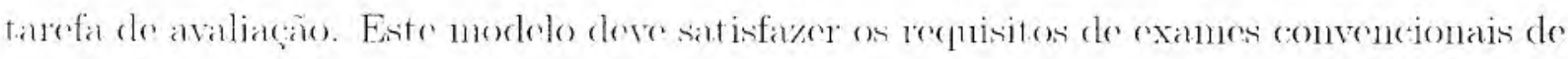

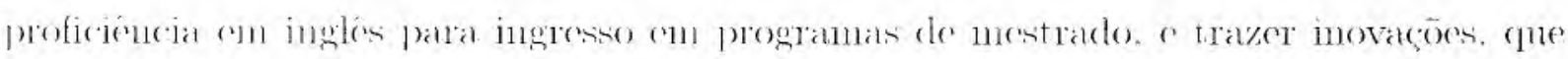
anxilieno o estuclante a desenvolver $u n$ escenema para o discuuso academico. tornando a tarefa de leitura de textos científicos mais eficiente:

b) desenvolver protótipos para avaliar antomaticannente exames com objetivos de

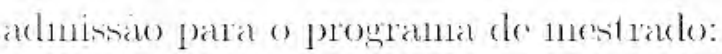

c) avaliar os protótipos no IC MC-LSP. em Sío Carlos. no programb de mestrado

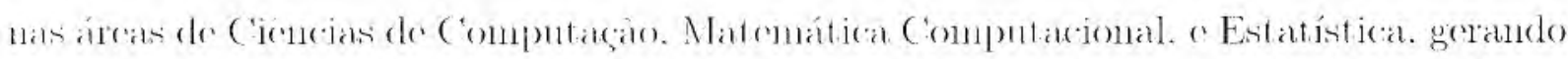
1111 sistenua rolusto:

(1) inplantar o sistona na estrutura organizacional do Programa de Mestrado do ICMC-LTSP.

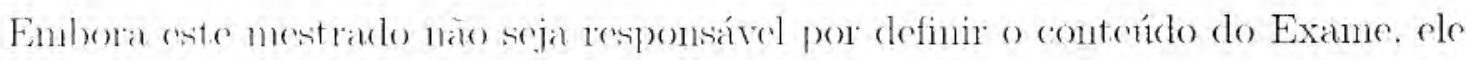
colaboron para o refinamento do exame proposto pela orientadora.

() presente traballuo está organizado da seguinte maneira. () Capítulo 2 descreve

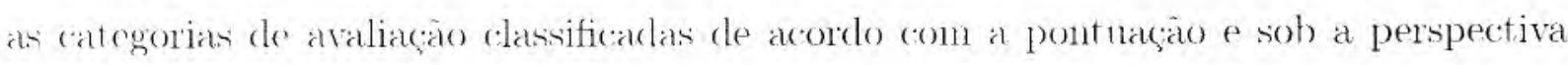
(lo propósito. () Capítulo 3 dencereve testes objetivos cufocando a stla construção e os 


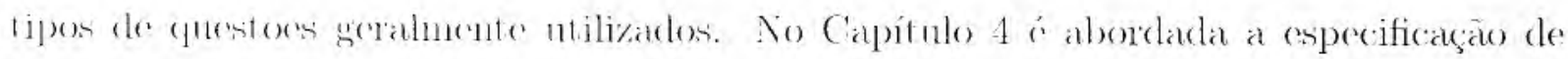

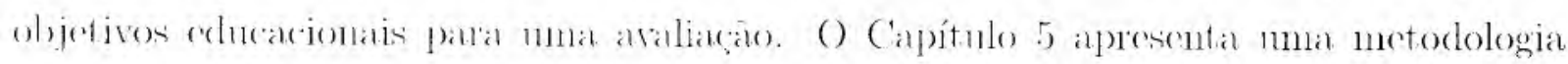

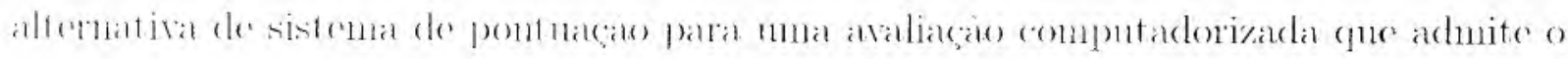

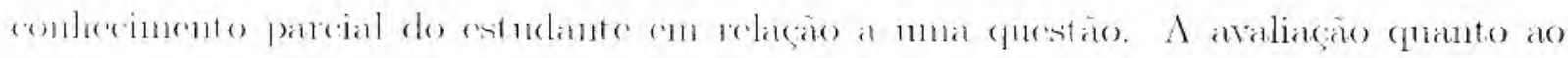

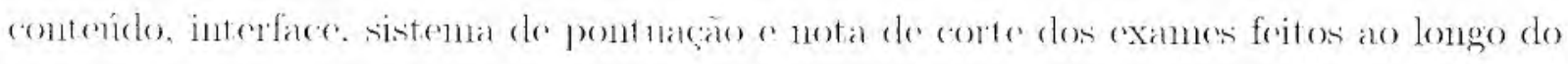
trabalho desenvolvido é apresentada no Capítulo 6. A descyicano do website gerado a partir do trabalho desenvolvido é descrito no Capítulo 7. Säo apresentados cinco apendices com exemplos de Exames de Proficiéncia elaborados durante o traballo. 


\section{Capítulo 2}

\section{Avaliação Acadêmica - Aspectos Gerais}

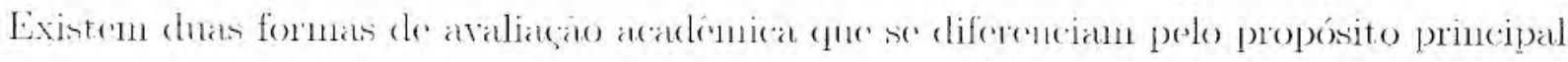

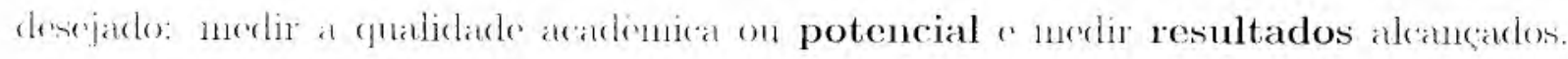

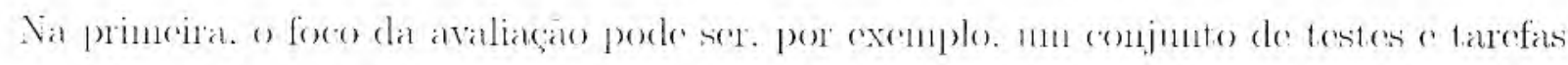

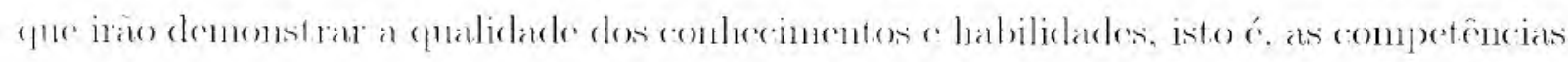

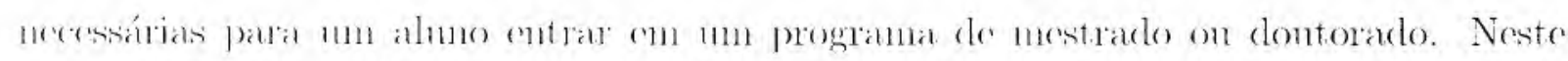

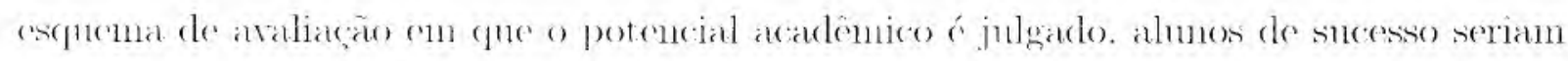

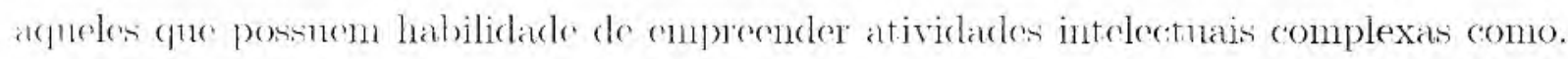
por exemplo. selecionar conherimente relevante de conterielos e habilidades aprendidos (oplicar este conllecimento para at solucão de algum problema. Os alunos deveriam fambrin ser (apazes do analisar " sintetizar a informaçà recuperada com ontros dados

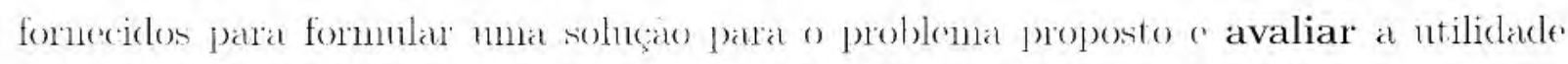

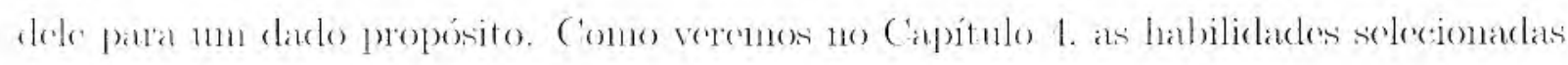

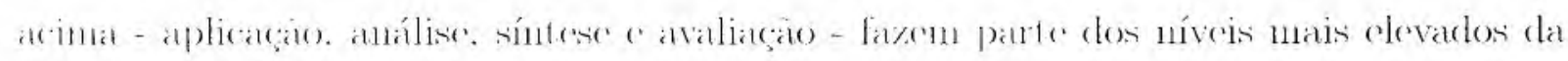

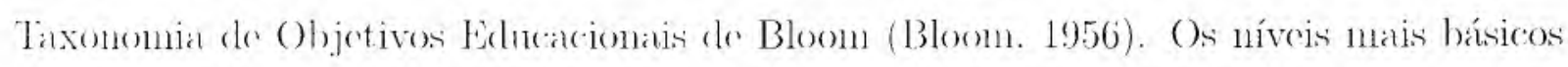

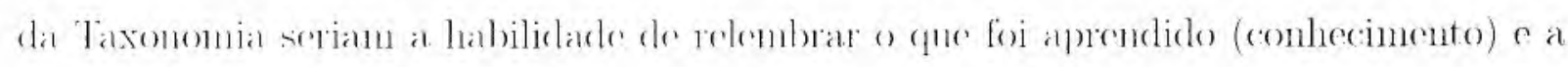

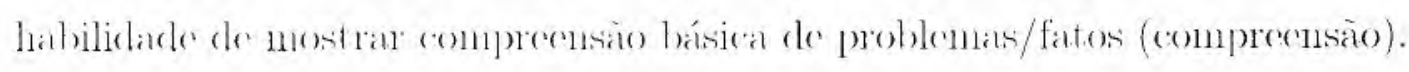

A seguncla forma de avaliacouo (une possui o popóxito principal de medir resulta-

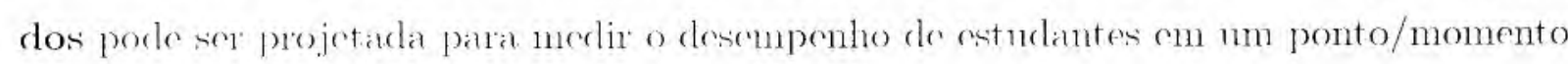




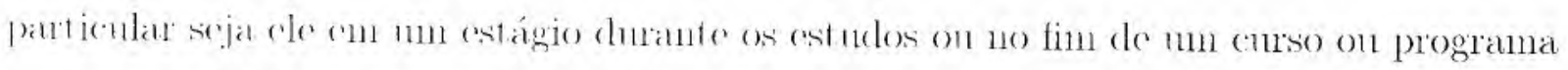

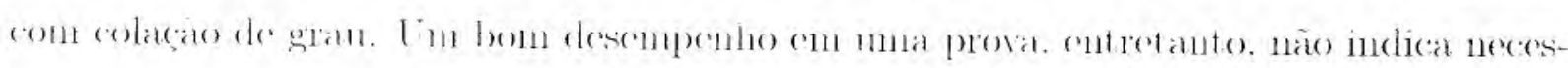

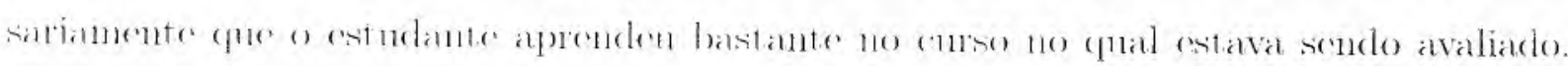

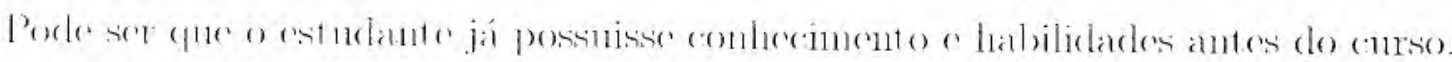

() noxo Exanne de Proficieneia rne Ingles (EPI) do ICMC-USP. que avalia habilidades necessárias para $110 \mathrm{n}$ estudante realizar o mestrado com snecsiso. se enequadra no primeiro dos doin propósitos descritos acima. Fle ocorre cun dluas épocas do ano abrile setembro). A pós-graduaça aconsella cure ele seja realizaro logo no início do programa de mestrado de forma que os almos postam conhecer o que se espera deles, com respeito a

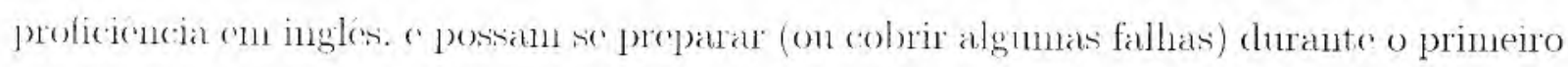

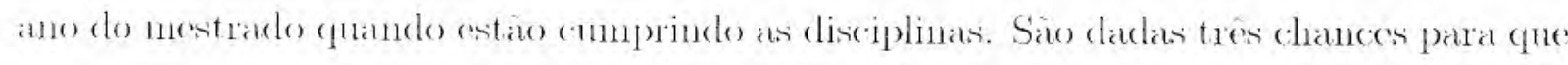

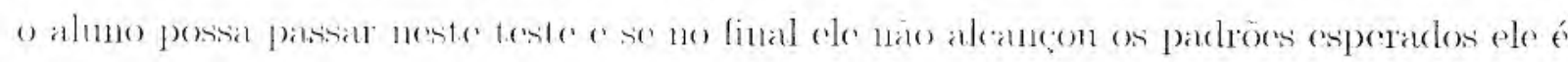
destigarlo do progranat de mestrarle.

\subsection{Categorias de Avaliação}

Alguns antores dassifican avaliaçào cle acordo com numa pontuaçăo pró-estabelecida versus

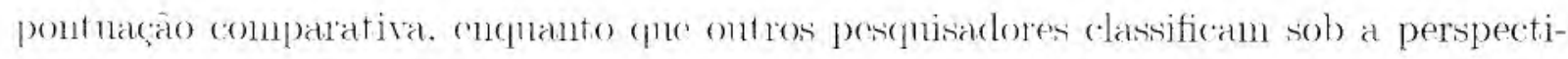

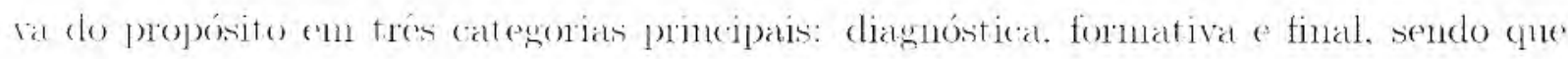

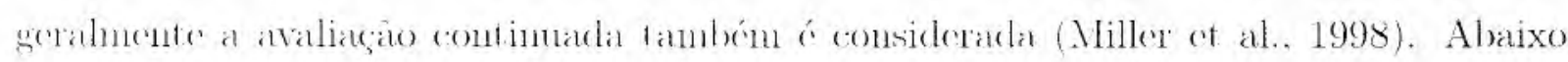

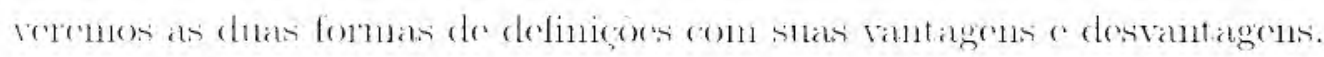

\subsubsection{Classificação da Avaliação de acordo com a pontuação}

\section{Avaliação com pontuação (nota de corte) pré-estabelecida (Criterion Refe- renced Measurement - CRM)}

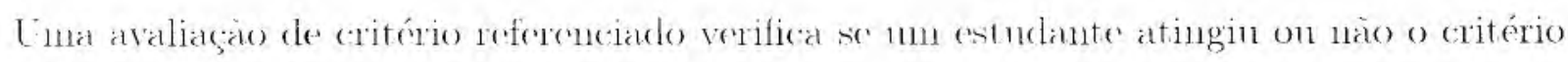

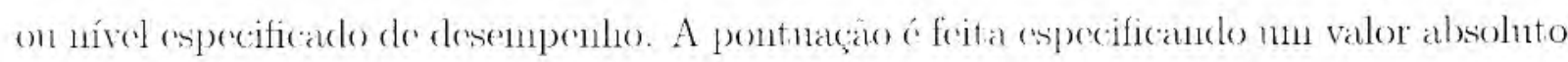

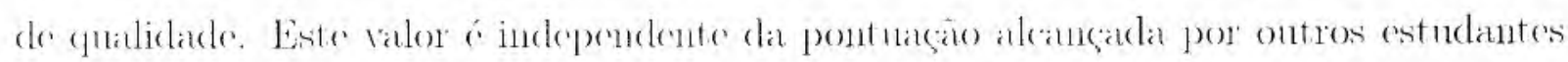

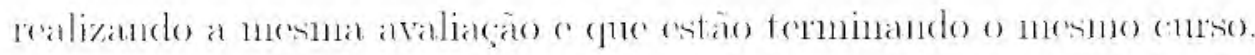

Fotat avaliaça cóntil para: 
a) Avaliar programas de aprendizado persomalizarlos:

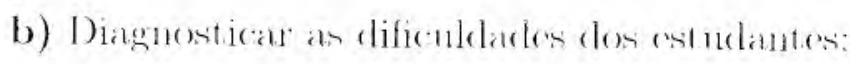

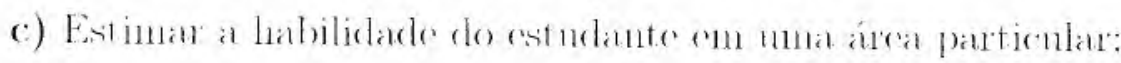

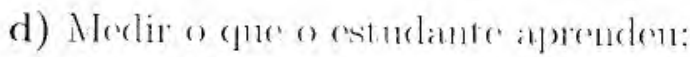

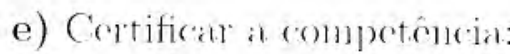

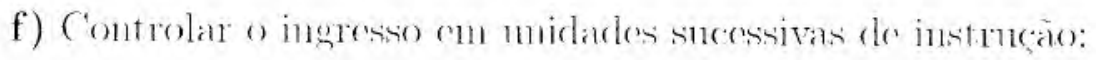

g) Sempre que o domínio de $m$ ansimuto on habiliclade é a preocupacào principal.

Porém existem as segnuntes críticas com relação a osse tipo de avaliação:

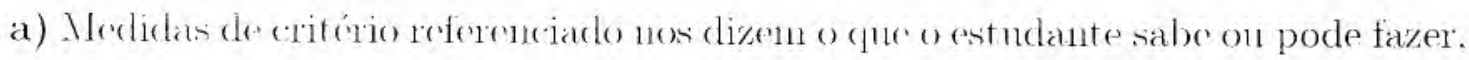

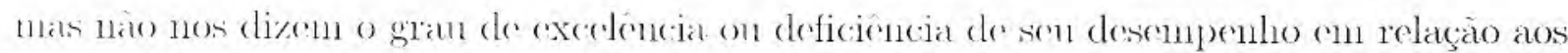
ontros cestudantes. Estes concritos hab relativos. por isso. mediclas de critério referenciado

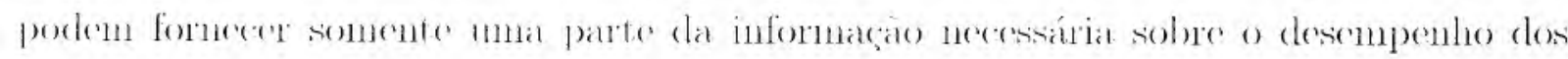
(estimlintits:

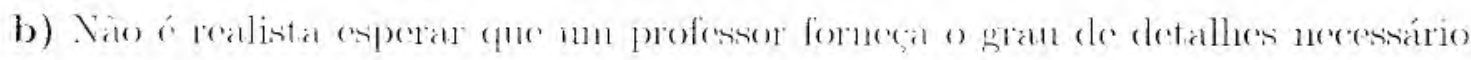

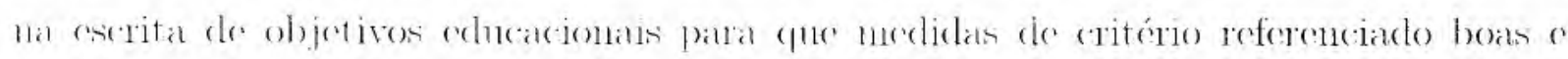
confiáveis postam ser obticlas:

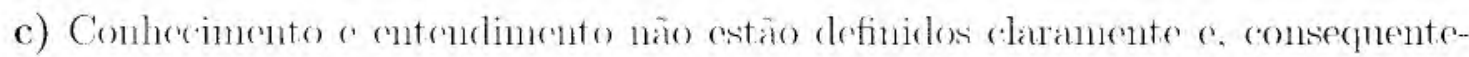

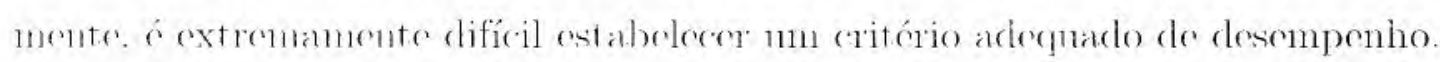

\section{A valiaçāo com pontuação (nota de corte) comparativa (Norm Referenced Me- asurement - NRM)}

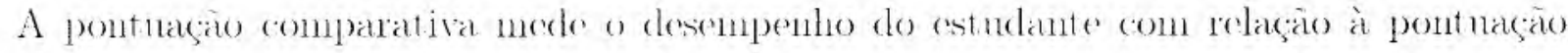

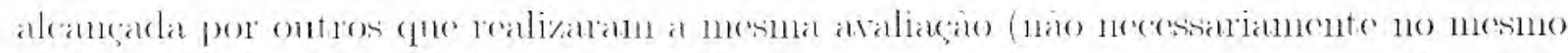
(1)111])

Esia araliacaro ó itil para:

a) Clasilicar estudantes:

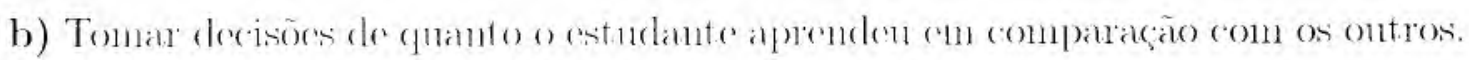

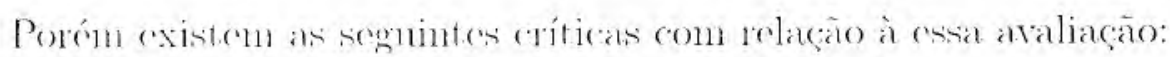

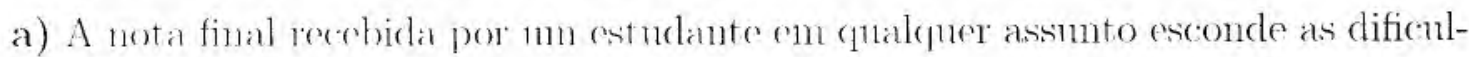

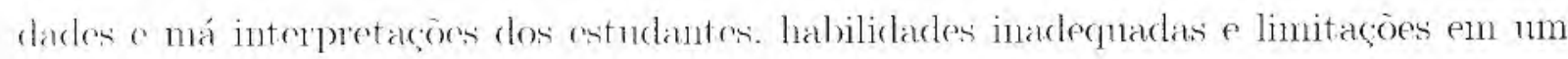


diato assiunto:

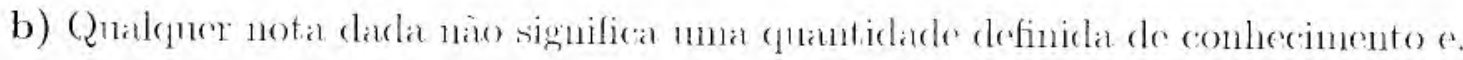

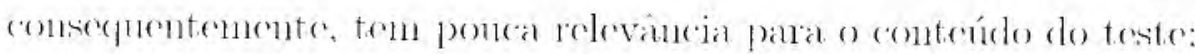

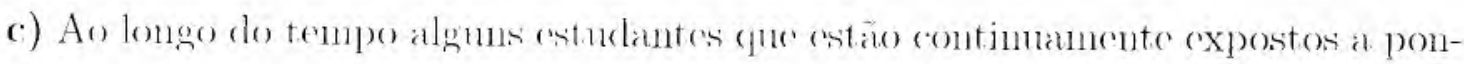

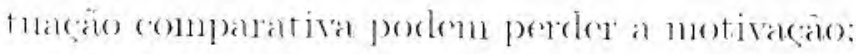

d) O uso de medidas de pontuaciono comparativa esconde o fato de que alguis aursos sào nuito bons cuquanto que ontros podem ser muito mins. on que professores ataledecen padroes rliferentes.

\subsubsection{Classificação da Avaliação de acordo com o propósito}

\section{Avaliação Diagnóstica}

Os testes do diagenóstico tôn como principal funçào posicionar adecluadamente os estudantes nos programas de ensino (pue serão disponibilizados. Por essia razão, esses testes são enupregados nos estágios iniciais do processo de ensino. preferencialmente antes do início dos estudos como nostra a Fignra 2.1. Dessa forma. o potencial (on a deficiência de cada

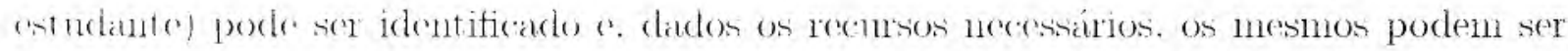

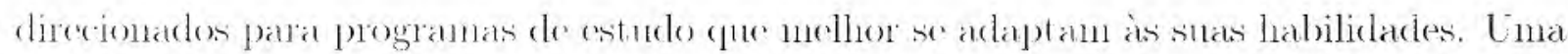

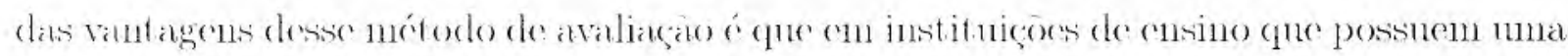

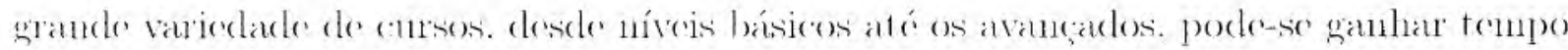

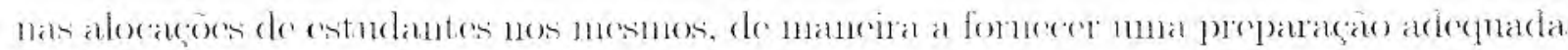

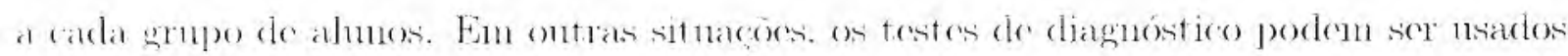

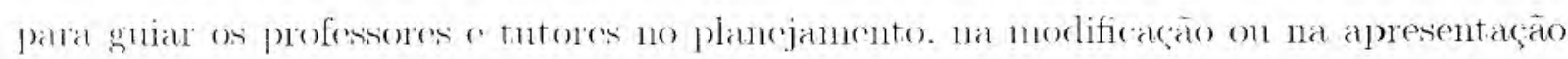
do conterído do enrso que melhor se arlapta à necessidlade dos estudantes. Assim. os testes de diagnóstion săo apresentados na forma de pequenos testes. freqü̈entemente de múltipla

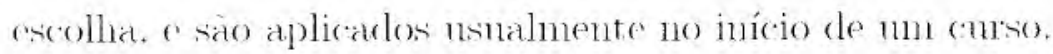

\section{A valiação Formativa}

Exrrécios, tarefas e testes progressives daulos aos estudantes durante nun ano on semestre

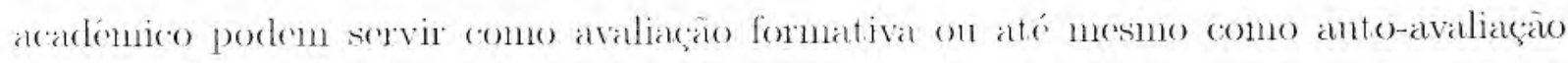
(Figura 2.2). A principal proposta da avaliaço formativa á fornecer mana retroalimentaçón 


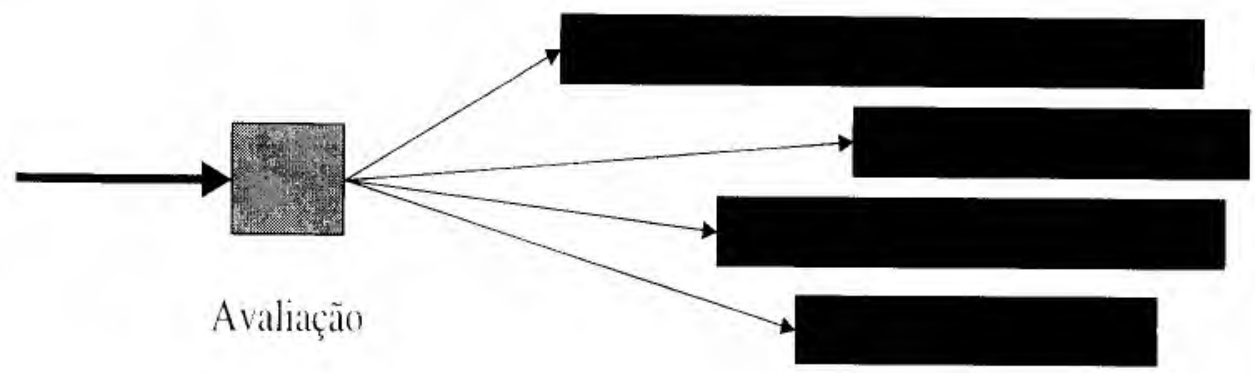

Programas de ensino

Figura 2.1: Avalialgion no início do procenso de ensino.

regular ans almuss. estimulando sern apremelizado e dando informacoes que os ajudam a julgar suas estratégias inclividuais de estudo. Ela também serve para alertar os professores

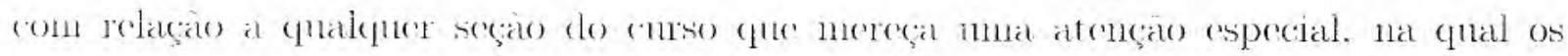

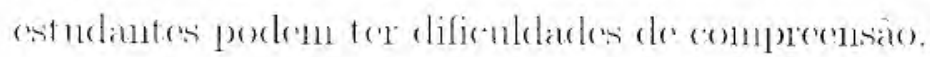

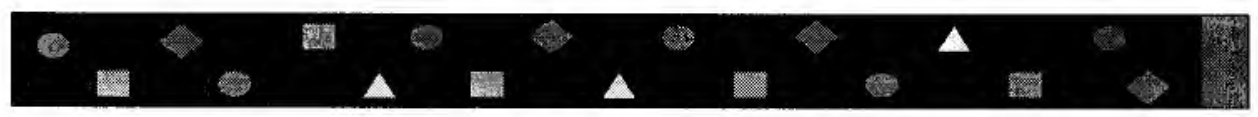

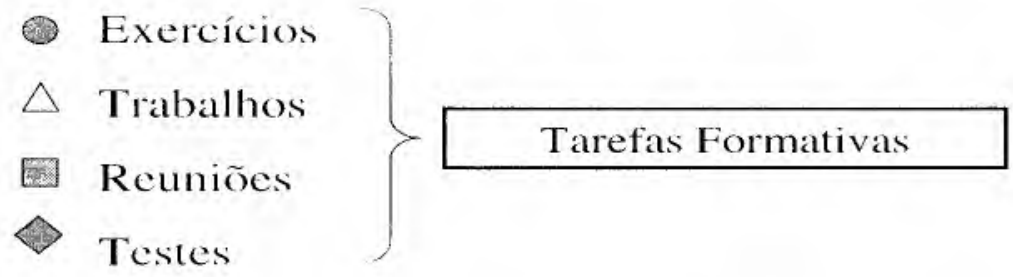

Fignua 2.2: Avaliacian Formativa.

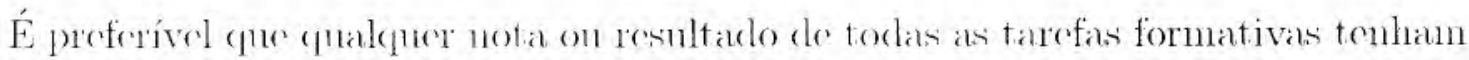

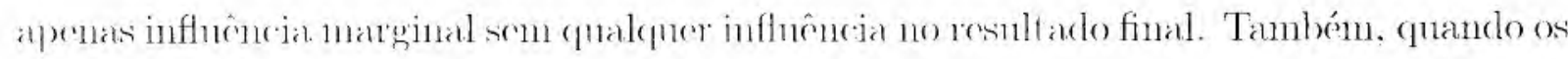

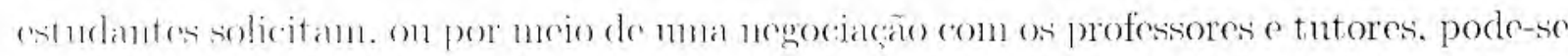
considerar os resultados das tarefiss formativas no resultado final. Há trôs importantes princípios gue devem ser observados:

1. ()s cstudantes devem ser informados quanto alos resultados logo que possível. A propestar da retroalimentaçấ f́ fornecer ans estudautes informagoes sobre sen nível

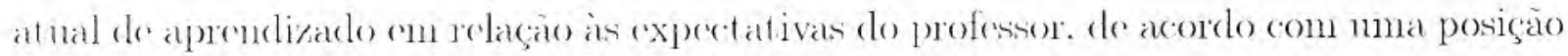


particular do estágio do conso:

2. () professores derem farer crílicas o comentários comstrutivos ans estudantes.

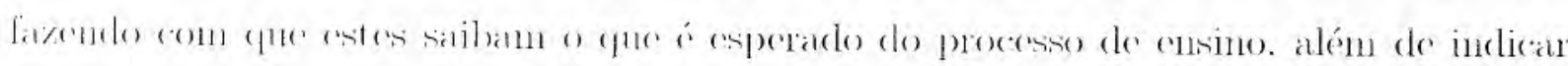

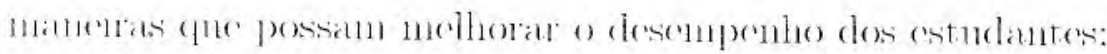

3. Os modelos de tamefis a trabalhos que teulanm bom desempenho devem ser formecidos ans alumos de maneira gue a hoa prática possal ser disseminada.

Portanto. o noso regular o adecpuado da avaliacaio fornativa a um importante com-

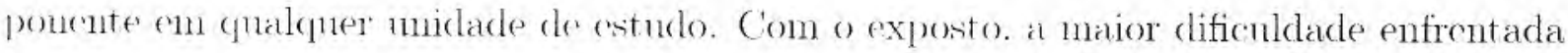
polos professores á julgar qual ó a melluor combinaçầo clas técnicas de ensino. de estudo e

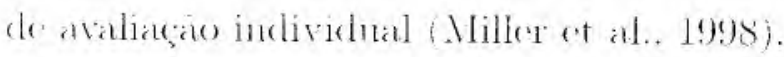

\section{Avaliação Final}

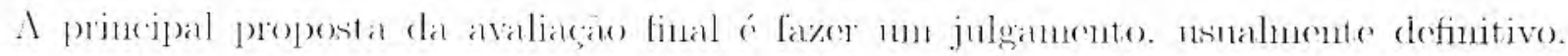

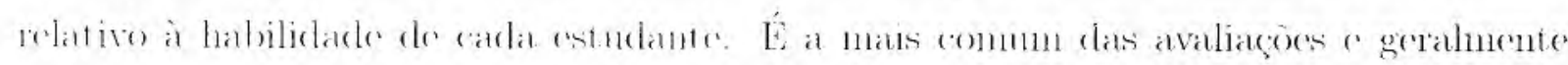

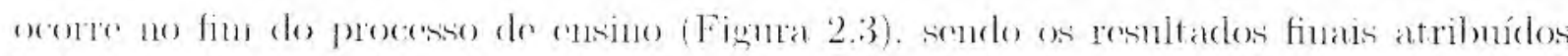
la forma de pontuagion (por exemplo: 7 pontos de 10). porcentagens (por exemplo: 65\%

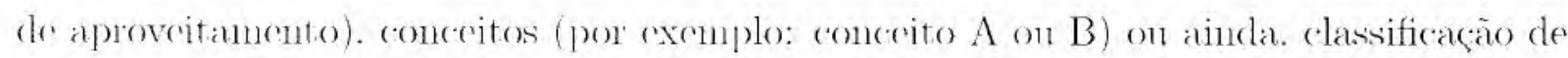
gran (por exemplo: (pualificado parar entrar 11111 gran superior de estudo).

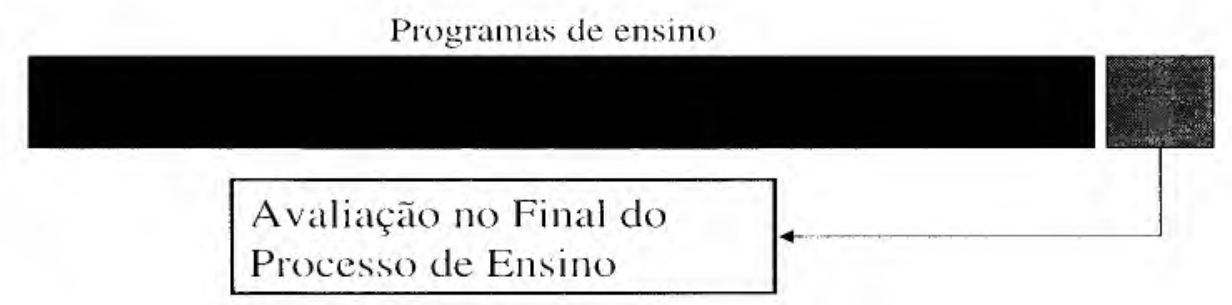

Figura 2.3: Avaliagaio Final.

A avaliascaso final pode também ser definida como nun instrunento de medida de

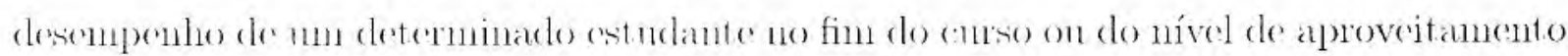

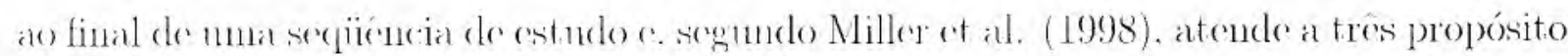

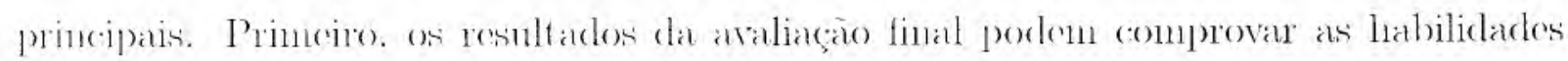

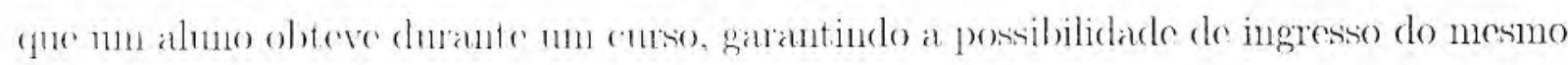




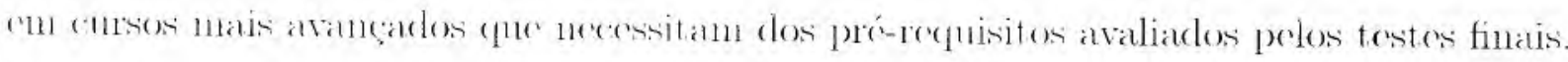

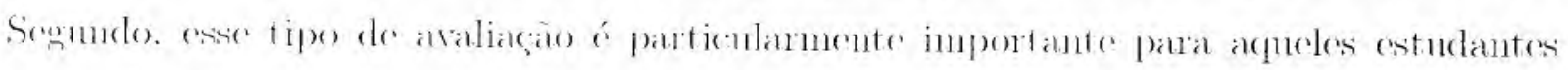

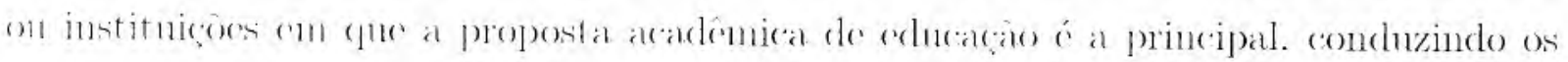

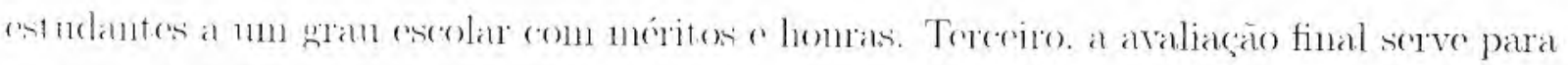

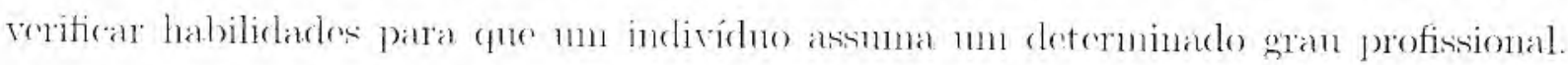

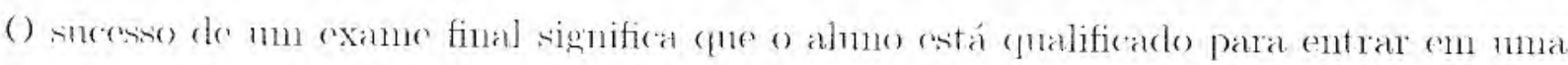
determinada profissáo.

\section{Avaliação Continuada}

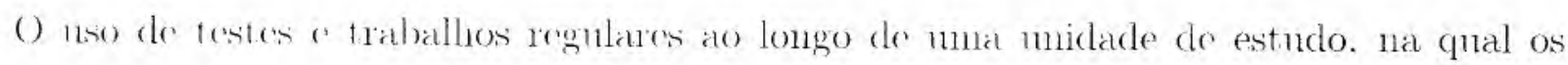

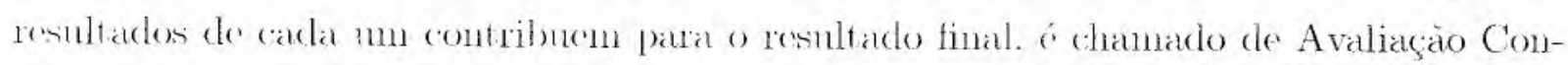

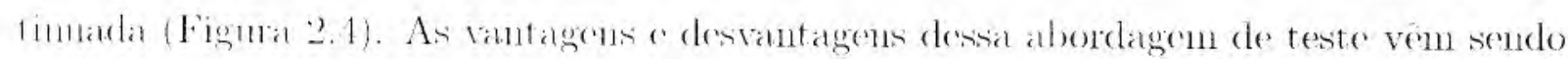

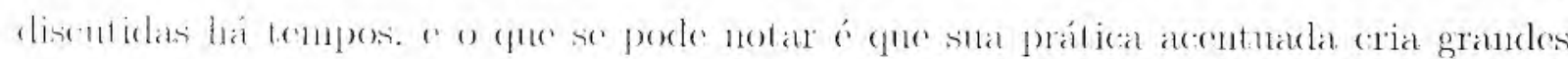
dificuldarles para estudantes o profensones.

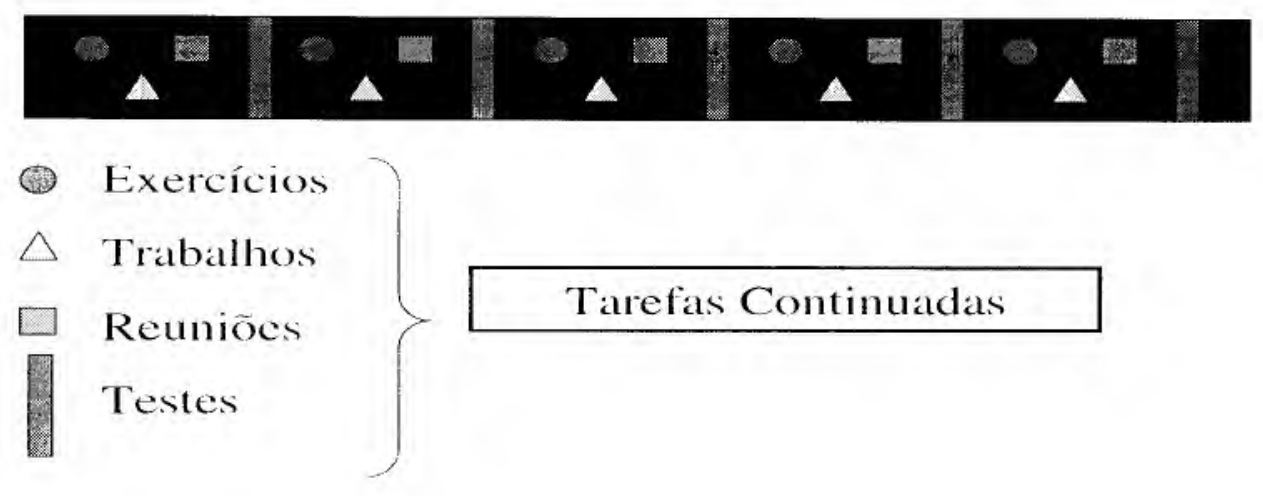

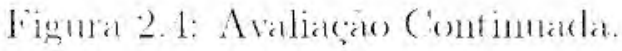

Tha das principais dificuldarles oncontradas na aplicação da avaliação continuada aparece quando silas tarefas comstantes se sobrepoem às atividades de ensino, sobrecarregando os estudautes a tal ponto (que naro possuenu mais tempo para estudar o assunto tratado en profiundielade (devido à ocomencia constante de testess). ben como limitando

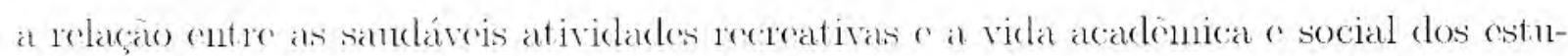

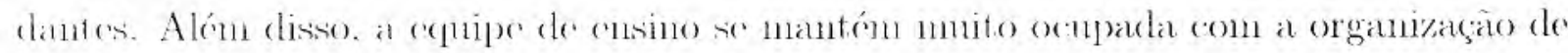

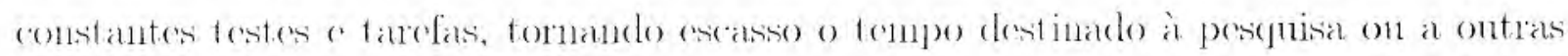




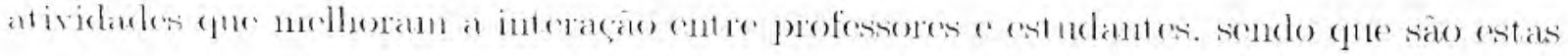

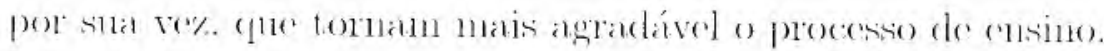




\section{Capítulo 3}

\section{Testes Objetivos Informatizados}

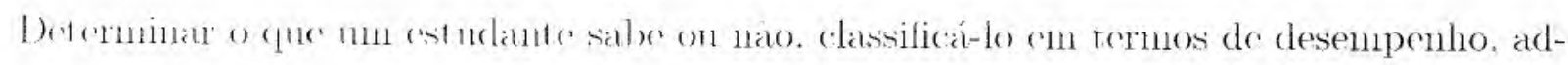

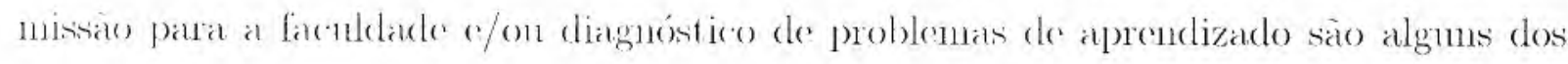
vários propósitos para os quais mula avaliagãos a ut ilizada. Independentemente da for-

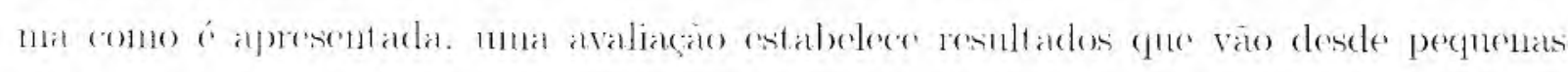
(o)

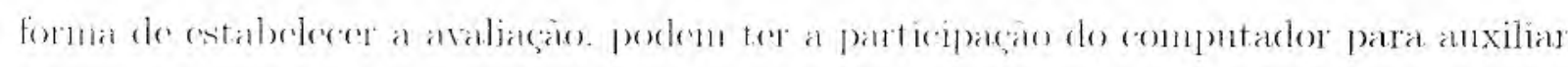

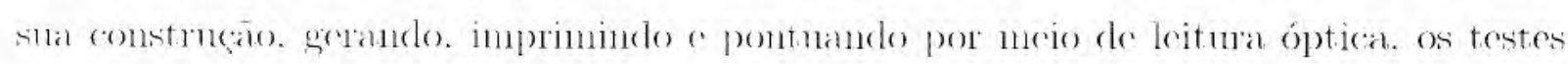

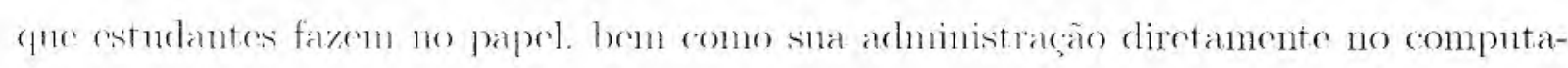
dor. Existenu vantagens o linnitacoés ent anbas as formas de participação do computador.

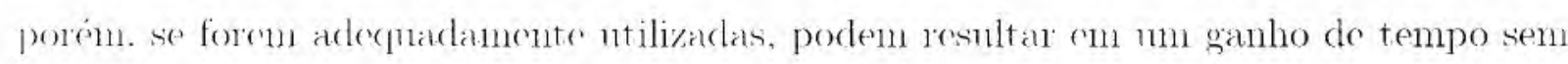

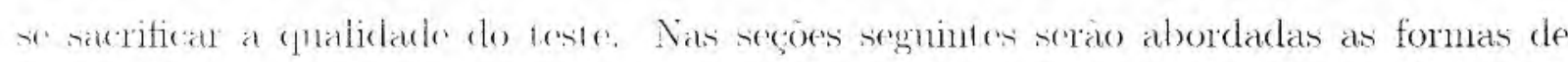

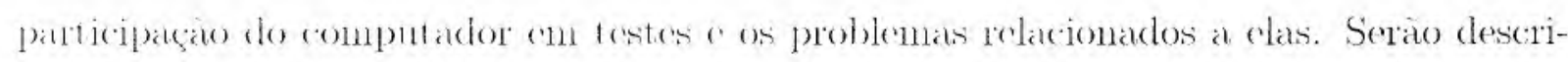

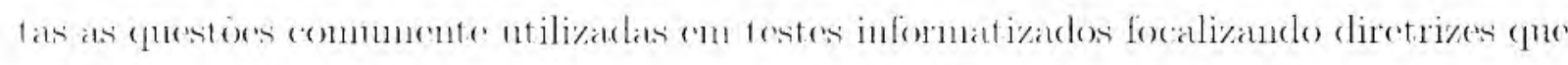
anxiliann suat (onstruçocs.

\subsection{Uso do computador na construção de testes}

Segunde Alessi d- Trollip (1991). os computadores podem anxiliar na construção de testes do várias formas. Uma prineira forma de anxílio é a criação de um banco de questöes. on

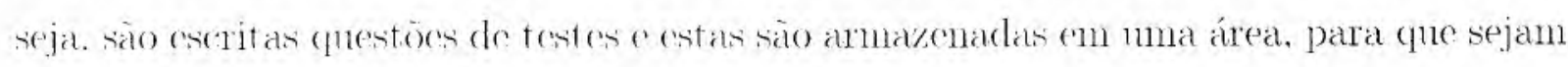




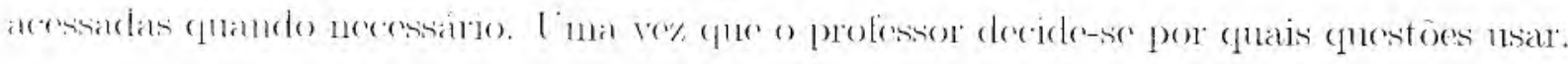

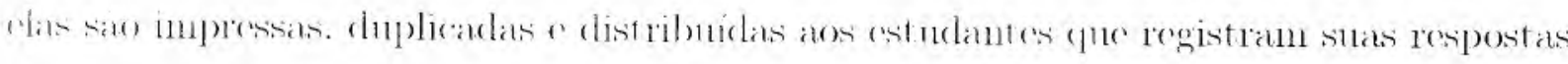

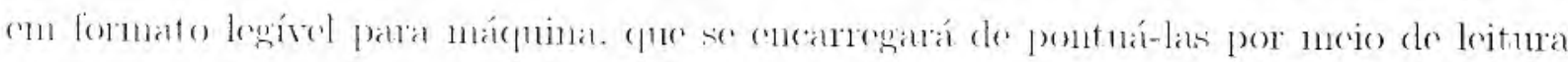
optica.

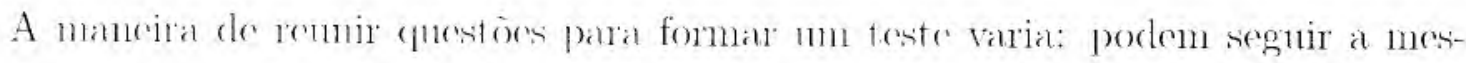

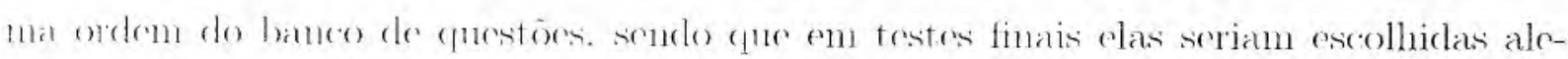
atomianente: podem sere ordenadas alcatoriamente para cada estudante. on seja. perma-

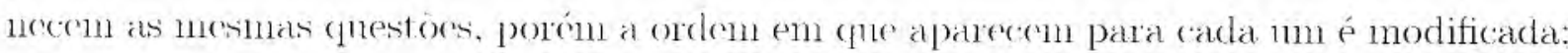
podem ser selecionadas de 110 grande pool. tendo cada estudante 1 min teste composto por questón diferentes.

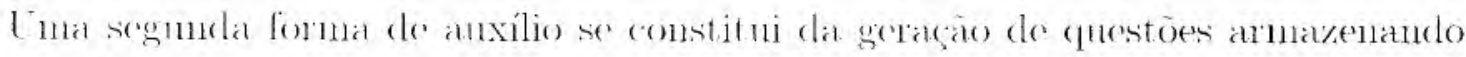

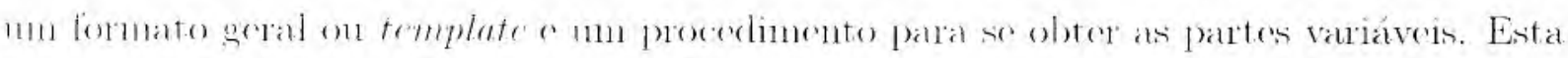

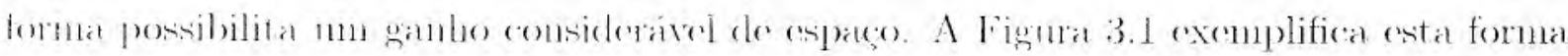

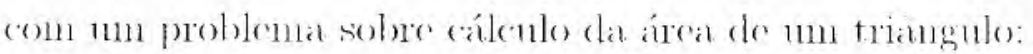

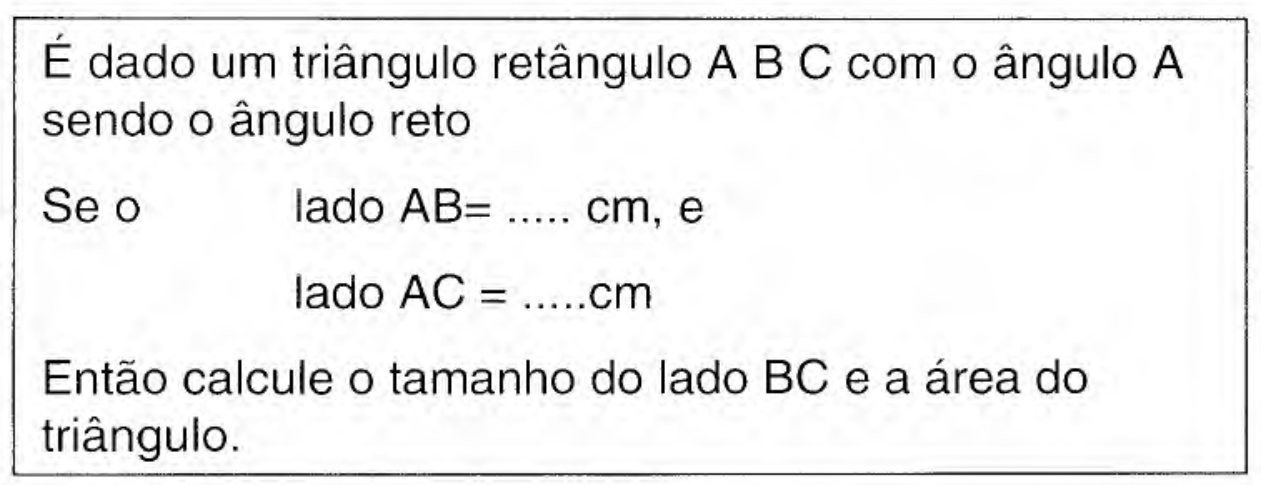

Figura 3.1: Tomplate para as (puestoes sobere triangules (Alessi aud Trollip. 1991).

Assinn. para gerar. por exemplo. I0) questoes a resperito da área de rum triangulo, seria necessírio armazenat apenas $u$ template como o da figina 3.1 e os procedimentos serian os responsáveis por fornecer as partes variáveis apresentadas na Figura 3.2.

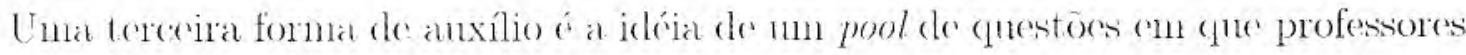

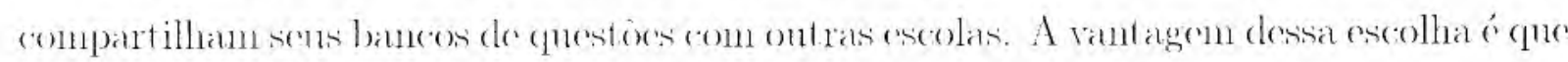

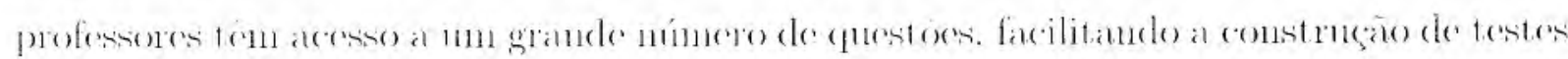

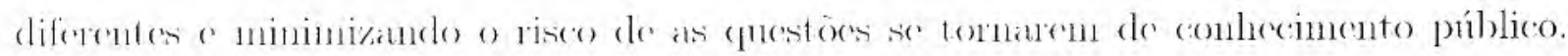




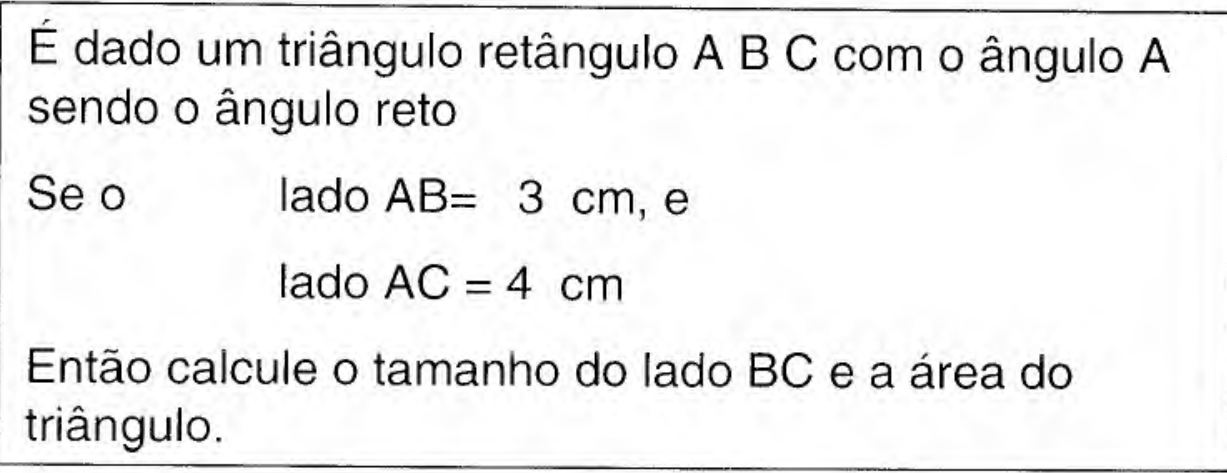

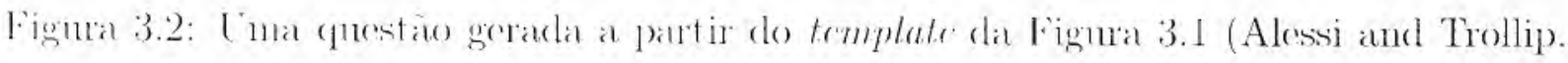
(1991).

Alén disso. a sobrecarga individual dos professoresés á rechuzida. pois a claboraçào de

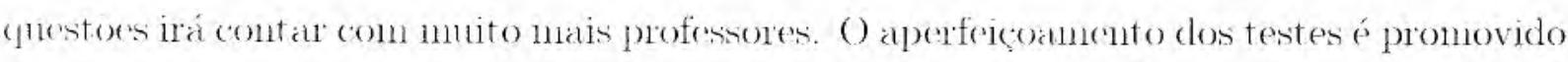

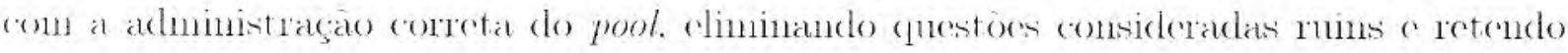

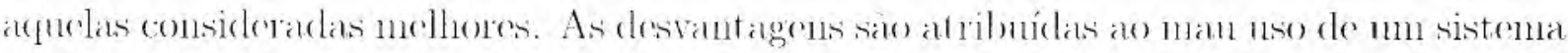

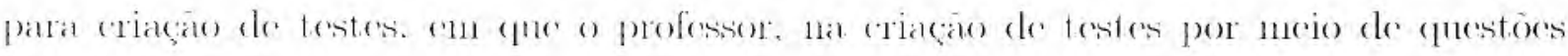

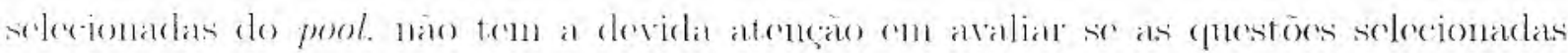

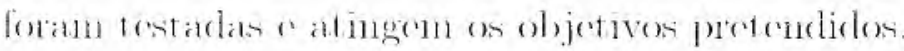

\subsection{Uso do computador na administração de testes}

En testes adninistrados por computador. o estudante atribui as respostas diretamente

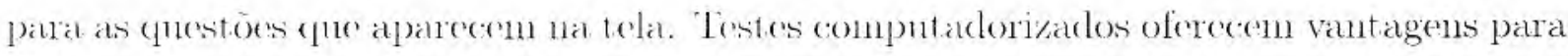

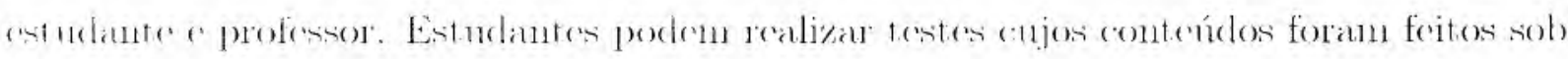

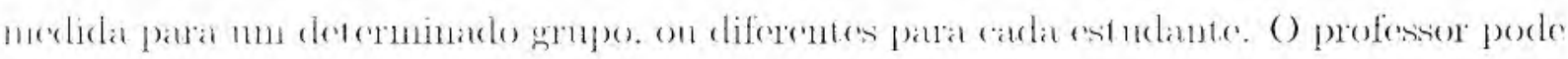

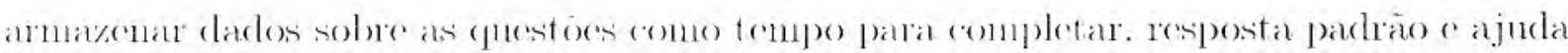

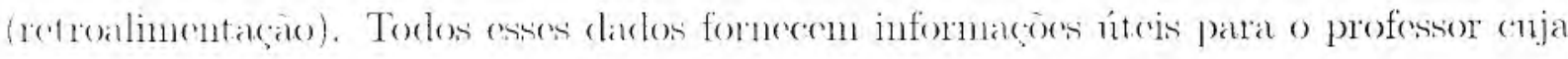

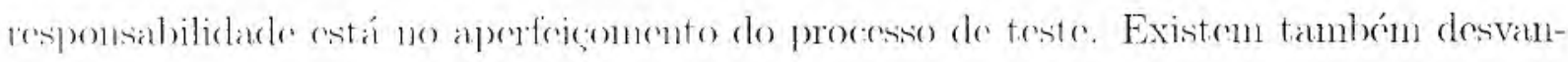

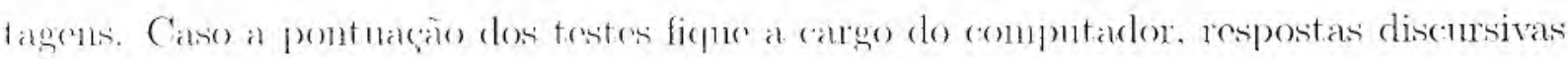

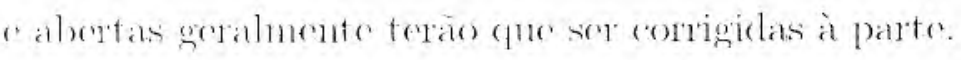

Administrar testes por computador acaba gerando vários problemas logísticos re- 
lacionates a:

- Número de computadores. Há a necessidade do grande quantidade de comprt-

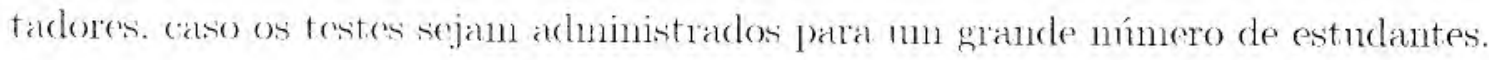

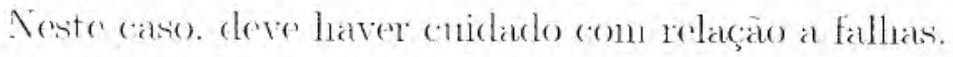

- Horários. Facilidades devenu ser providas para fure os estudantes tenham seris iesters em horários flexíveis coscollhidos por eles.

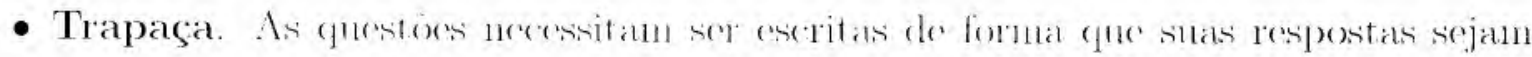

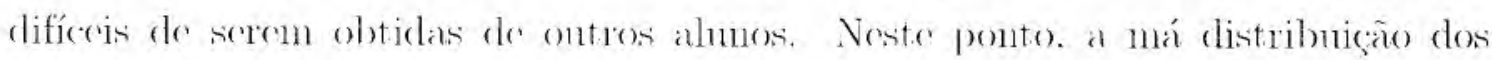
computadores na sala em que o teste será aplicado porle facilitar a prática da cola'.

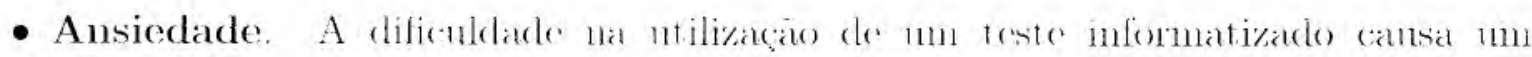

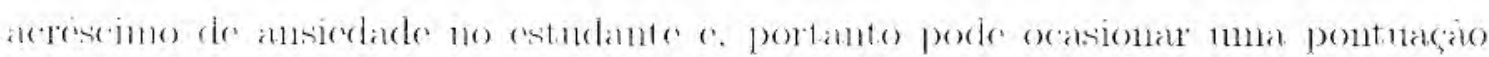

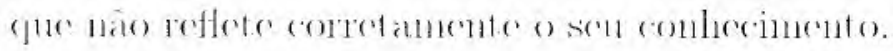

So o teste informatizado for ben projetado, a tendéncia. geralmente, é que os

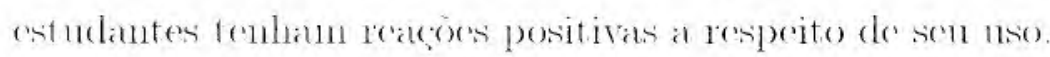

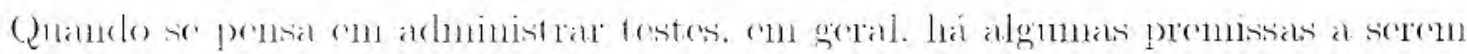

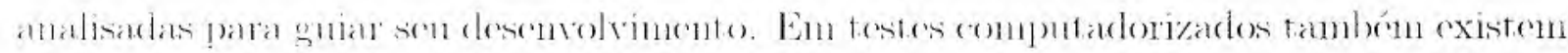

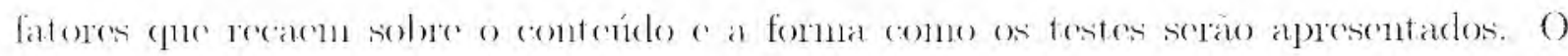

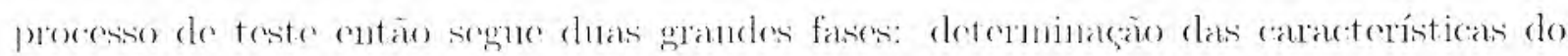

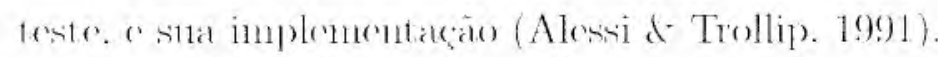

\subsubsection{Características do teste}

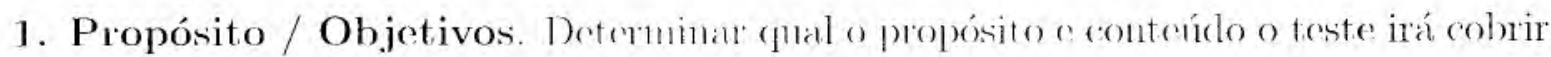

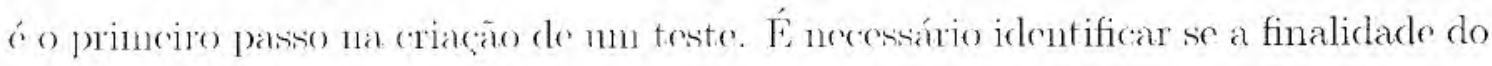
teste ć diagnesticar o nivel de conlereimento do estrudante no sentido de encaminhá-

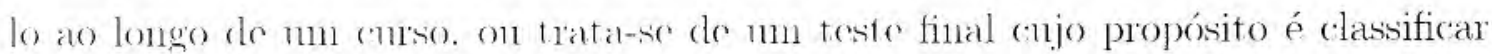

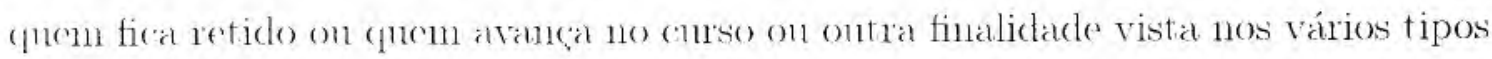
de avaliaço do Capítulo 2. () 1uesuno teste. porén. pode ser usado para propósitos

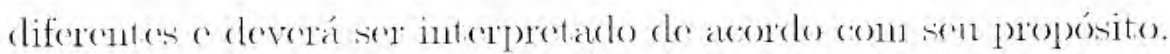




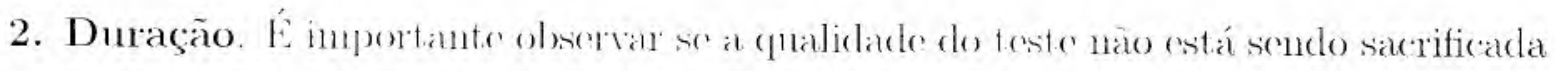

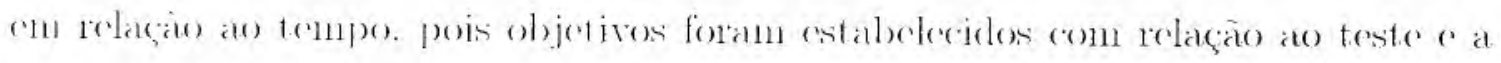

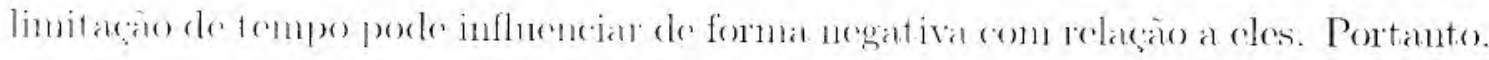

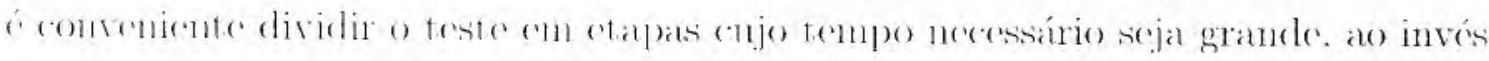

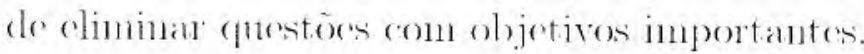

3. Pool de Questões ou Geração de Questões. Nil seguio 3.1. vinnos três maneiras

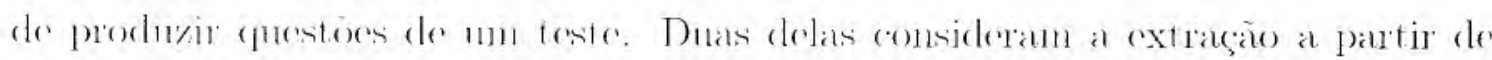

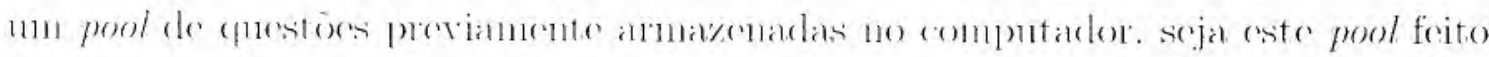

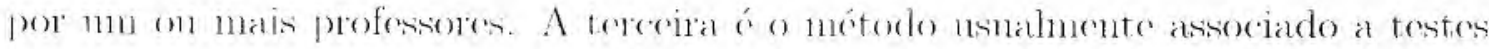

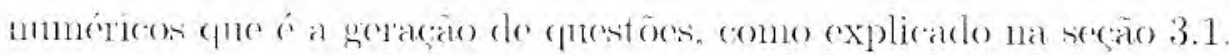

4. Tamanho do Pool de Questões. Grandes pools promitemn muna maior variedade e.

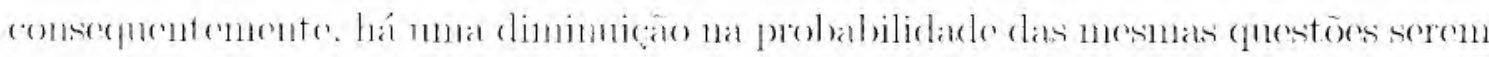

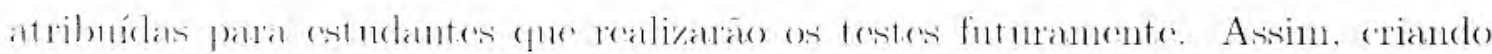

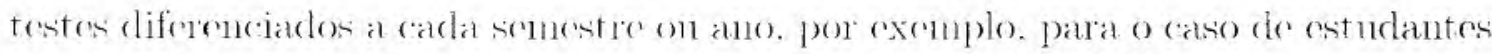

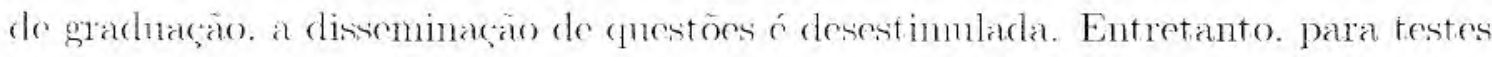
informais, nào existe a neessidarle de um graurle pool.

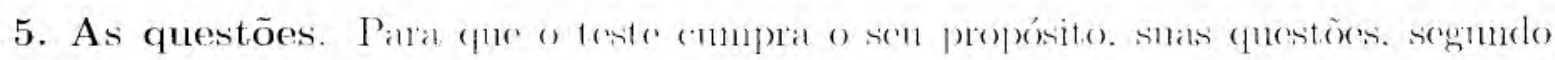

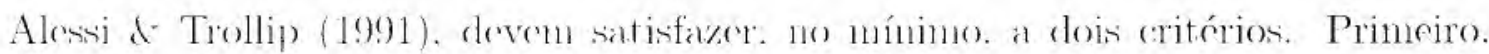

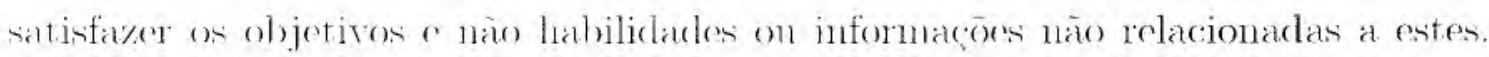
Veste casose a familiaridade com o computador pode ser uma cunestäo crítica pois pessods que se sentem intinidadas podem ter sen desempenho afetado pela ansiedade

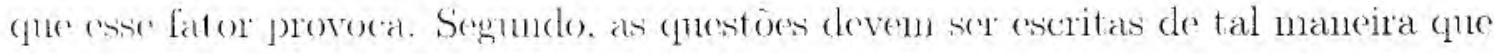

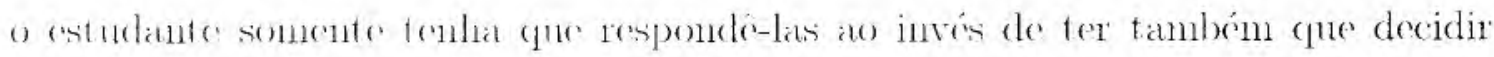

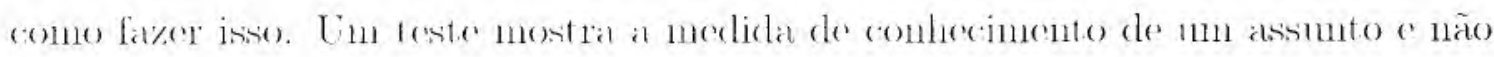

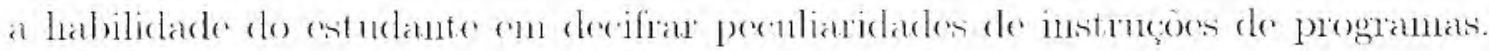

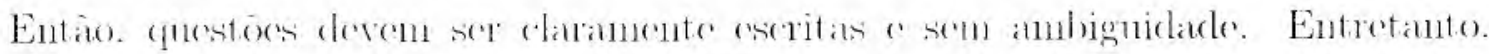

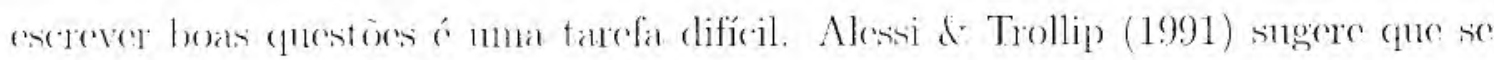

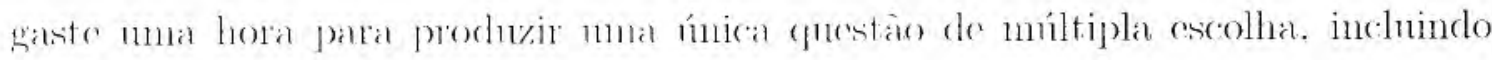
escrever. testar o revisals. 
6. Retroalimentação. Dependendo do tipo de leste a retroalinemagào pode on nào

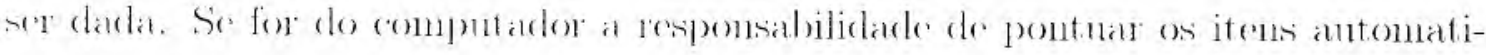

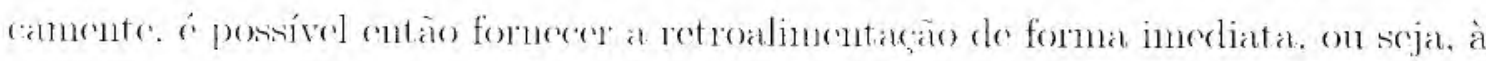

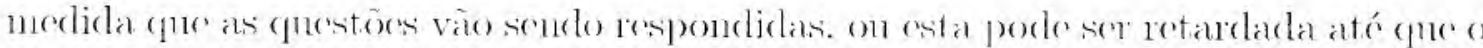

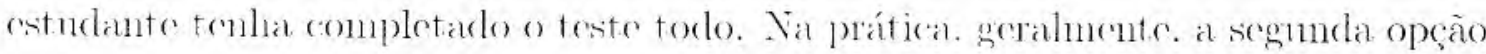

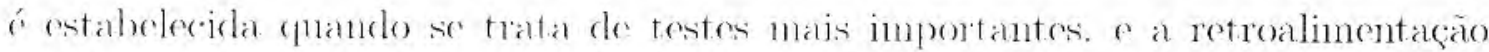
inediata a ntilizada om testen menos formais.

()utras caracteristicas a serem observadas a respeito da retroalimentacão referem-se ao contendo. que pode indicar apends correçầo (o que f́ correto on incorreto) on pode formecer também num explicaräo. Segundo Alessi de Trollip (1991). como o

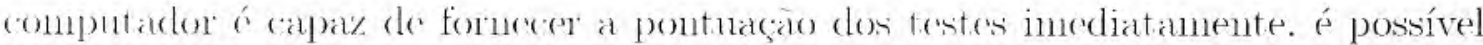

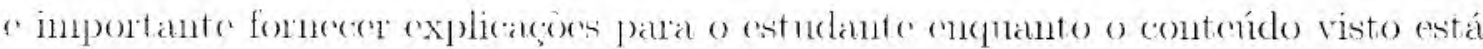
"freseo" nal stlal Hormórla.

7. Pontuação Necessária. Se for apropriado para o propósito do teste. podo existir 1 ma "nota de corte" o esta vai variar segundo a findaliclade do teste. Algmunas vezes. mun teste pode nato ter nota de corte por exemplo. (quando a intenção é localizar

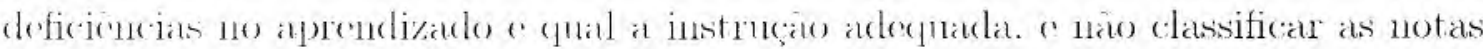
finais dos astuclatulas.

8. Cronometragem. Alessi d Trollip) (1991) argunentann ser contra testes que

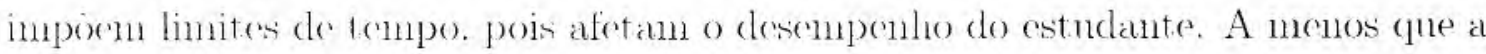

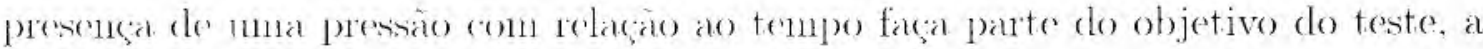

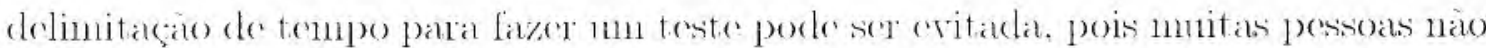

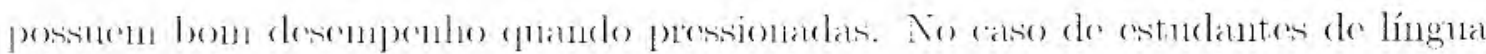

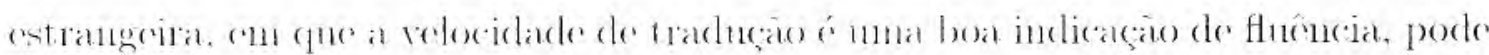
se dizer (que a presisio do tempo far parte do tester.

9. Dados Coletados. Pontuacào, respostas indivicluatis de cada questão, tempo ntili-

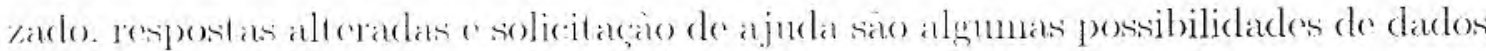

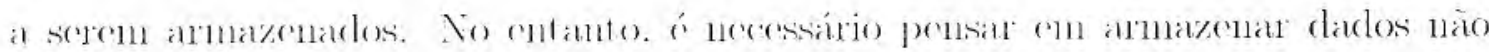

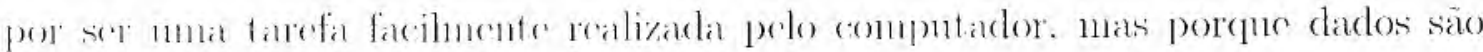




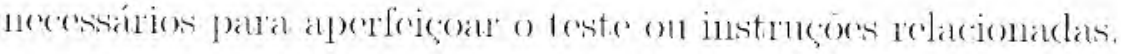

10. Apresentação dos resultados. Resultados podem ser apresentados de duas for-

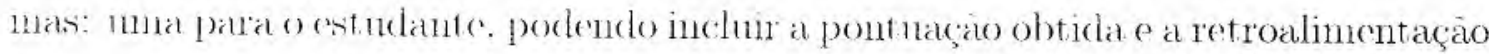
contra para o professor. inchundo normalmente os l'siltados de todos os estndantes (c smonários ustatístions.

\subsubsection{Implementação do teste}

() prontos abordados anterionmente estan relacionados conn as características dos testes.

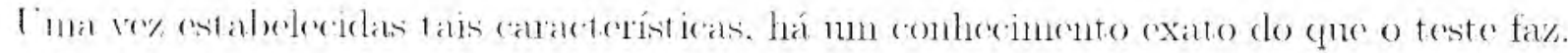

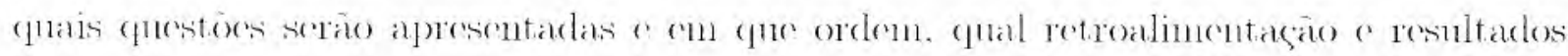

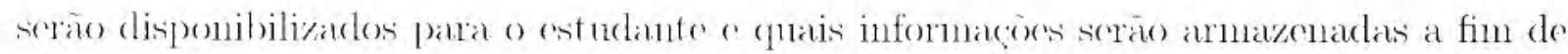

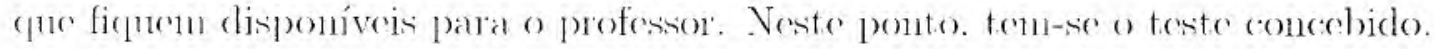

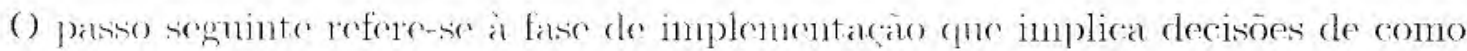
vismalizar o teste na tela. como operá-lo. quais opecoes estarăo disponíveis para professor

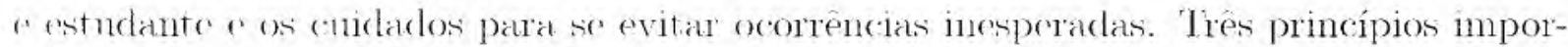
tantes nerecenn ser observados para qualquer tipo de teste a ser projetado:

- Assegurar fácil acesso a informações necessárias. Para que o lisiário tenha acesso fácil às informacôes necessárias. f́ preciso deridir quais são essas informaçôes. () professor. por exenuplo. necessita de acesso fácil aos resultados. enquanto cune os estndantes necessitam de direcionannento de como ó o nliso do sistema de teste on como as questoes necessitan ser respondidas.

- Maximizar o controle do usuário. Maximizar o controle do nsilário (estudante on professor) significa permitir tomada de decisáo sobre o próximo passo a ser feito r quando fazê-lo. on seja. liberelade enu "navegar" no sistema.

- Controlar a segurança e estabelecer redes seguras. Este princípio refercsc aos cuidados a serem observados no intuito de evitar ocorrências acidentais que veruham a danificar informaçoes do sistema de teste. Para isso é necessário investigar quais atifudes o nstánio pode ter crue levem a algum dano frente ao sem desempenho no teste. tentando estabelecer barreinas que evitem tatis at itudes. 


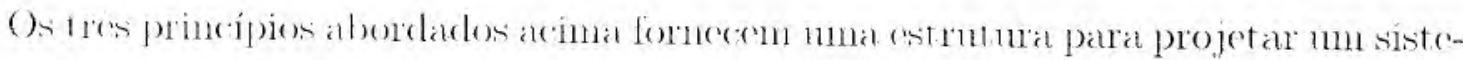

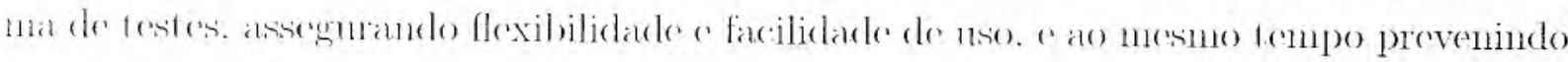

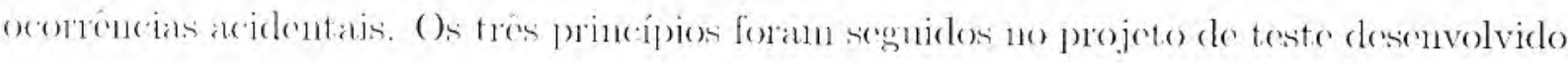
para o Exane de Profieciencia en Ingles do ICMC-LSP (veja Capétulo 7). Basseado nestes

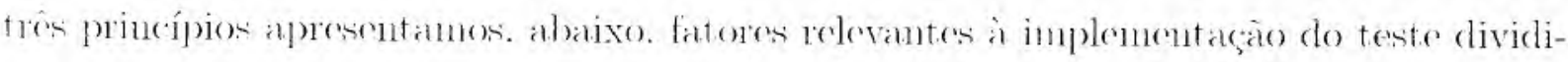

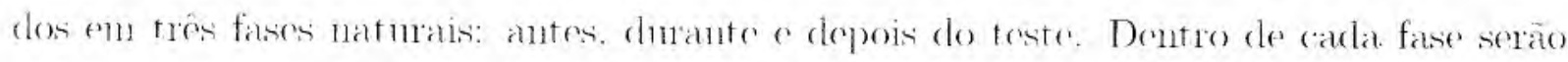
examinados os papéis do professor re do estudante no processo.

\section{Antes do Teste - Papel do Professor}

Este ć o momento em que o professor cstabelece parametros para o teste. Além

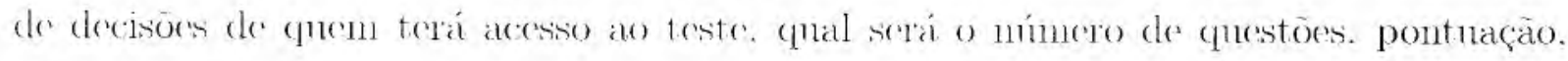

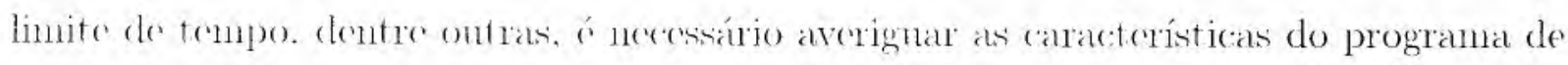

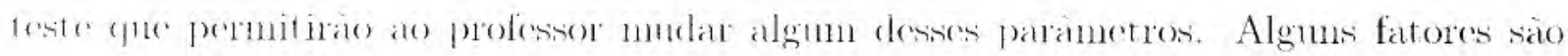
deratharlos abaixo.

- Assegurar fácil acesso a informações necessárias. Asscgurar que o estundante esteja fizendo o feste correto. bem como detcrminar se o teste esteja sendo feito

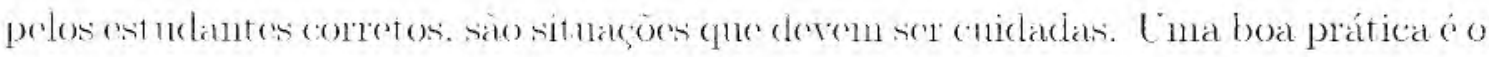
progranna do teste solicitar informacoes a respeito de cueme está acessando e checar

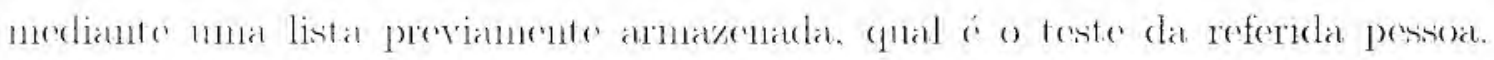

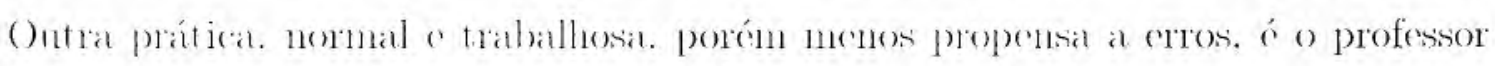

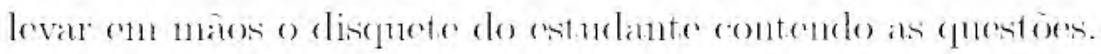

- Maximizar o controle do usuário. Alguns sistemis de teste permitem ao pro-

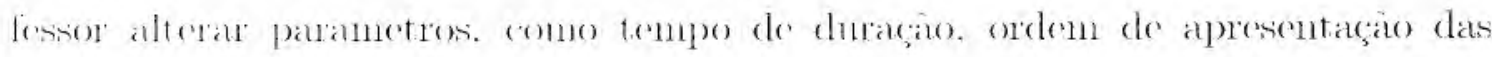

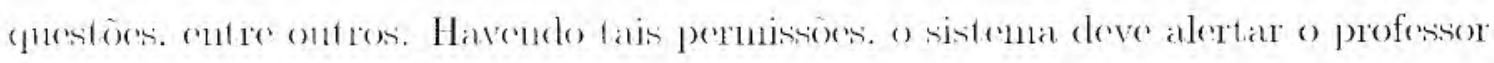

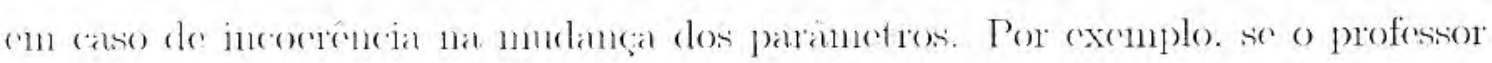

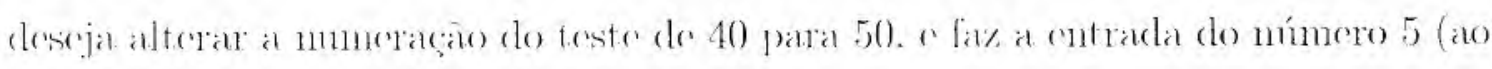

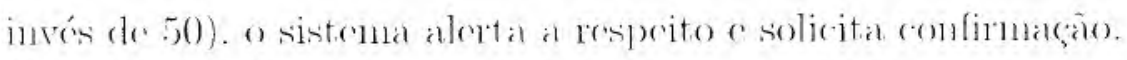

- Controlar a segurança e estabelecer redes seguras. É necrssário (fure o professon avalie o teste antes do o cestudante nutilizitolo. Esta prática assegura que todos

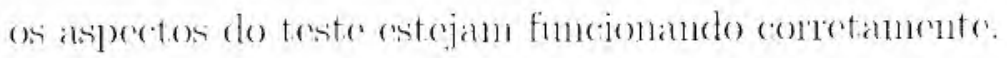




\section{Antes do Teste - Papel do Estudante}

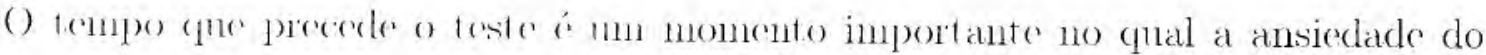

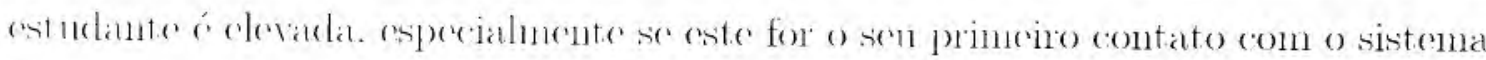

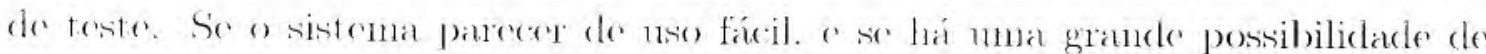

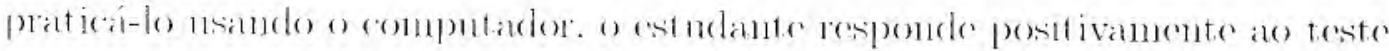

- Assegurar fácil acesso a informações necessárias. Thros tipos de informaçoes

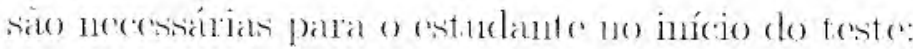

- Solore como ntilizar o computarlor co sistema de teste:

- Solne algunas restricós. (o)mo tempo permitido. (flando a cromometragem será iniclada o recentros permiticlos:

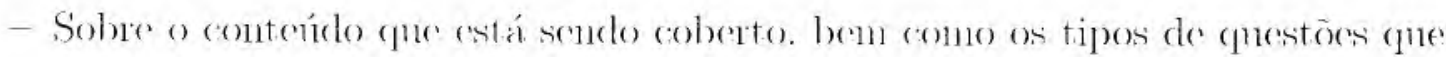
estain no tester e pontuaçäo necessária.

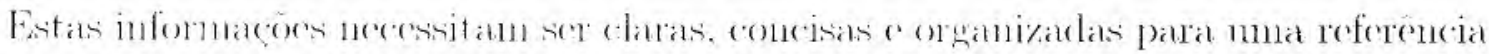

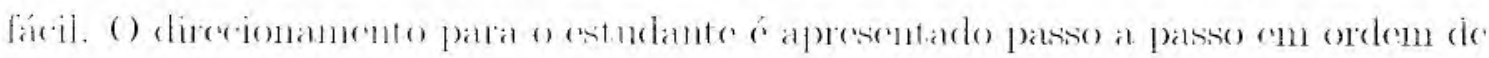
oromencia foralizando as informacoes mais inportantes.

- Maximizar o controle do usuário. A prática perle ser estabelecicla por meio de

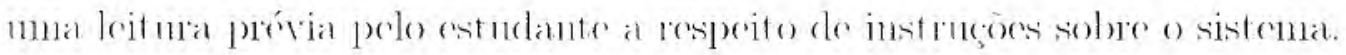

- Controlar a segurança e cstabelecer redes seguras. Sào necessários, ainda.

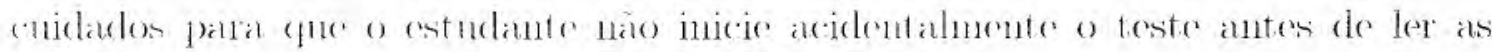

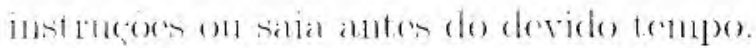

\section{Durante o Teste - Papel do Professor}

Váo lá tauto traballog. duraule o teste a ser feito pelo professor. Esta fase requer consideracoses a resperito de problemas gure aconterent durante a administração do tritie.

- Assegurar fácil acesso a informações necessárias. A trapaça e o término acidental sào chuas sitnaçoes que poden refuerer a intervençào do professor. Na

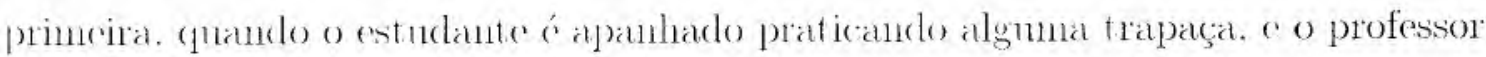




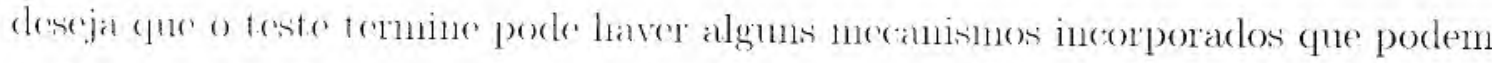

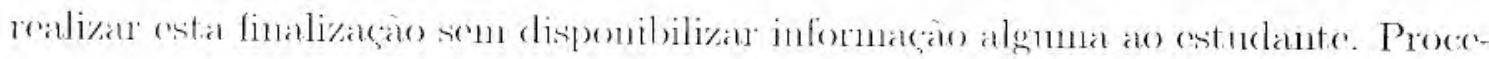

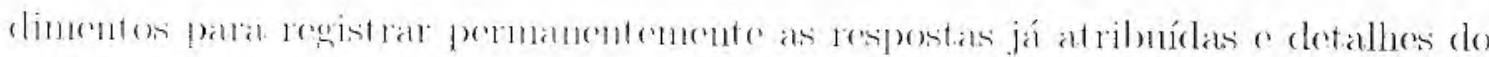

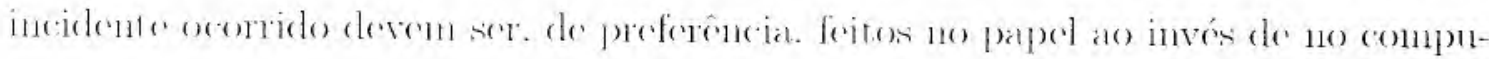
tador.

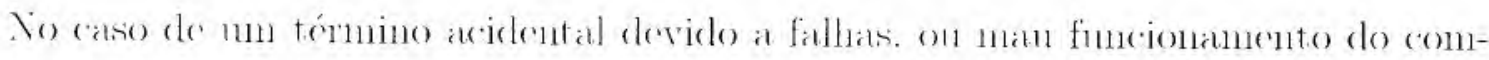

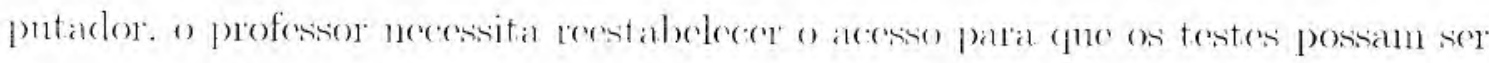

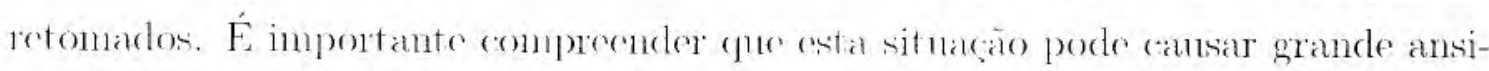
edade so năo mammscada corretamente. A intolerância do 1 mu estudante, que estava ghase finalizando num teste. frente a tal inciclente poder ser aliviada se todas as

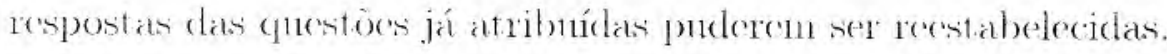

\section{Durante o Teste - Papel do Estudante}

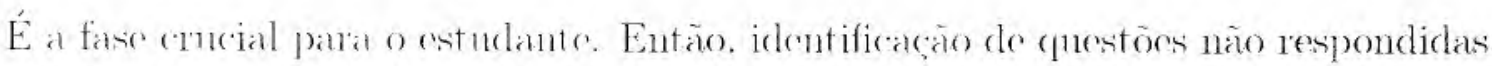
6 tempo restante. (aso seja inposto. sao informacoes que devem estar legivelmente disponíveis e facihnente acessiveis.

- Assegurar fácil acesso a informações necessárias. A flexibiliclade com relaçâo às respostas ć ontro ponto a ser tratado. Pode ser dada a opcrĩo de responder on não quando mua questão é apresentada. Caso não descje respondê-la, o estudante pode rutao navegar por todias as questoes antes de responde-las. Em testes adaptativos.

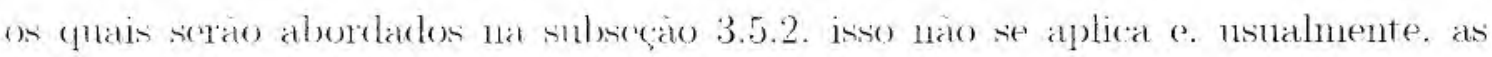

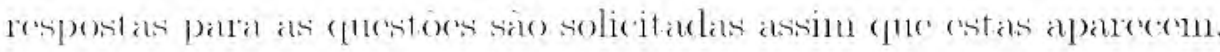

- Maximizar o controle do usuário. Em testes convencionais esta navegaçăo é desejável. () estudante porke descjar. ainda, alteran as respostas enquanto a questäo está na tela. on depois de ontra cunestão ter sido respondida. Permitir que ele possa

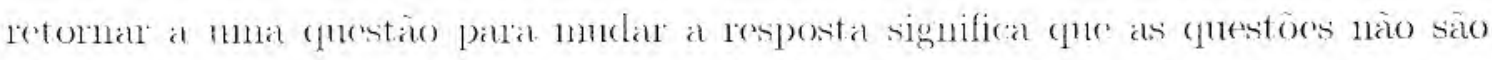

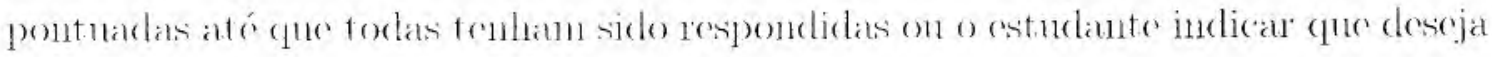
(ontinilli1:

- Controlar a segurança e estabelecer redes seguras. Acidentes conn relação

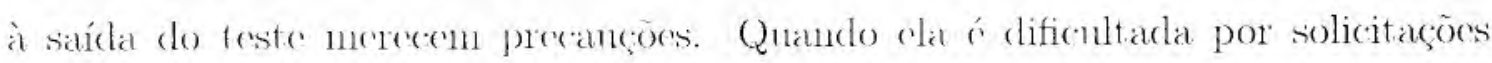




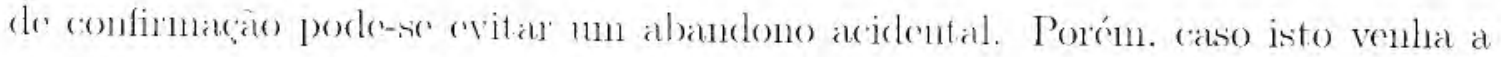

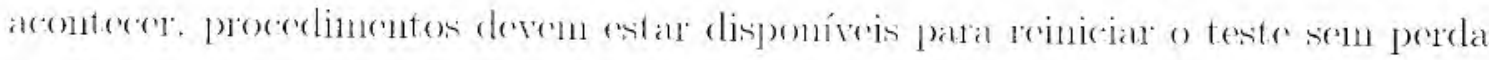

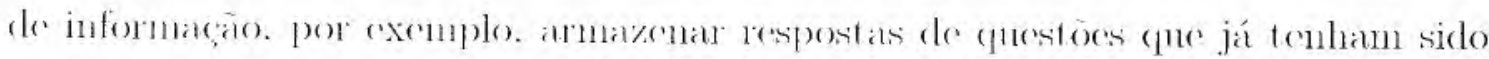

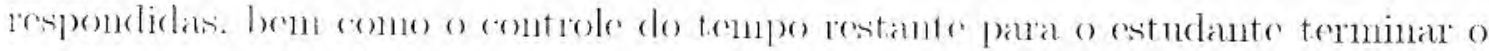

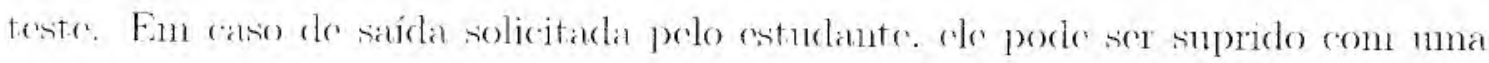
lista de questón que ainda não tenham siclo respondiclas. tomando então. a decisão de retomar as questöes on sair realnuente.

\section{Depois do Teste - Papel do Professor}

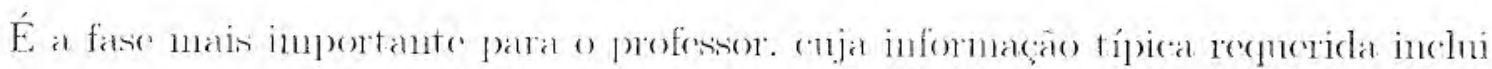

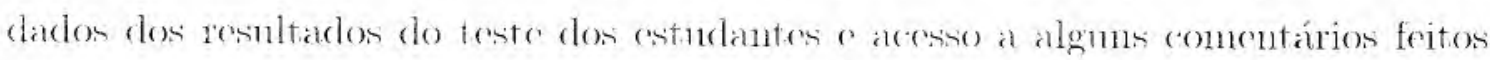
por estess.

- Assegurar fácil acesso a informações necessárias. () acrsso a esses clados deve

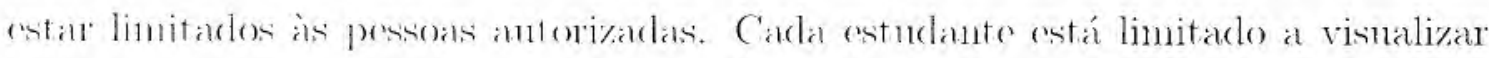

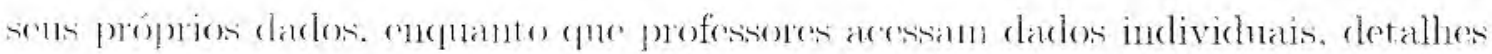
de rada (purstäo on andat mu resumo do desempenthe do grupo.

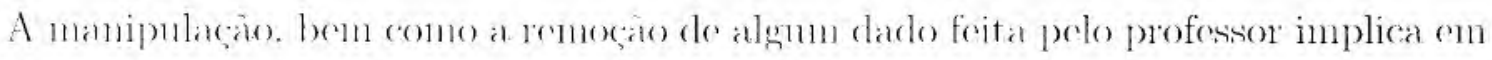

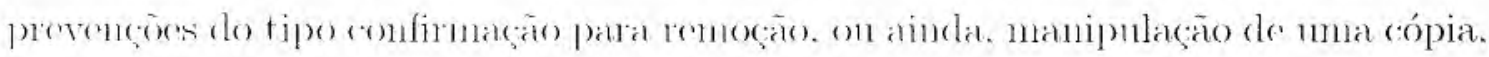
para se manter os dados originais intactos se honver necessidade de alteraçoes para propósitos estatísticos. por exemplo. A saícla dos resultados pode ser via tela on em papel. A segunda opecaro pode ser estabelecida para todos os testes que vão sendo

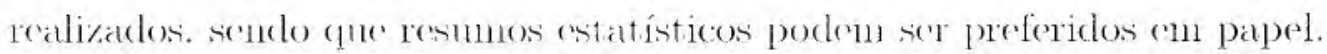

\section{Depois do Teste - Papel do Estudante}

- Assegurar fácil acesso a informações necessárias. ()s resiltados. con retroalinentadăo apropriada. sân as informacoes mais importantes e necessárias ao estudante após emupletan o treste. Muitos tostes tem registro do desempenho do estudante no exante. Eles podent. entän. acessar informaçoes rolativas a si mesmo. 


\subsection{Estrutura das Questões}

As curestón, enu geral. sào compostas pelas segunites partes:

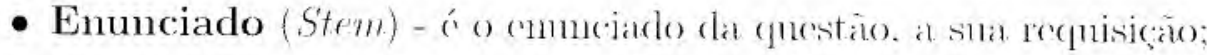

- Opções (Options) - sato as alternativas corretas e incorretas de respostas fornecidas após o crumuciado:

- Chave $(K e y)$ - ć(sao) a(s) resposta(s) correta(s) da lista de opçoes:

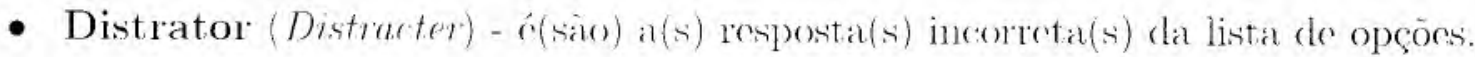

A Figma 3.3 excmplifica estas partes 1.

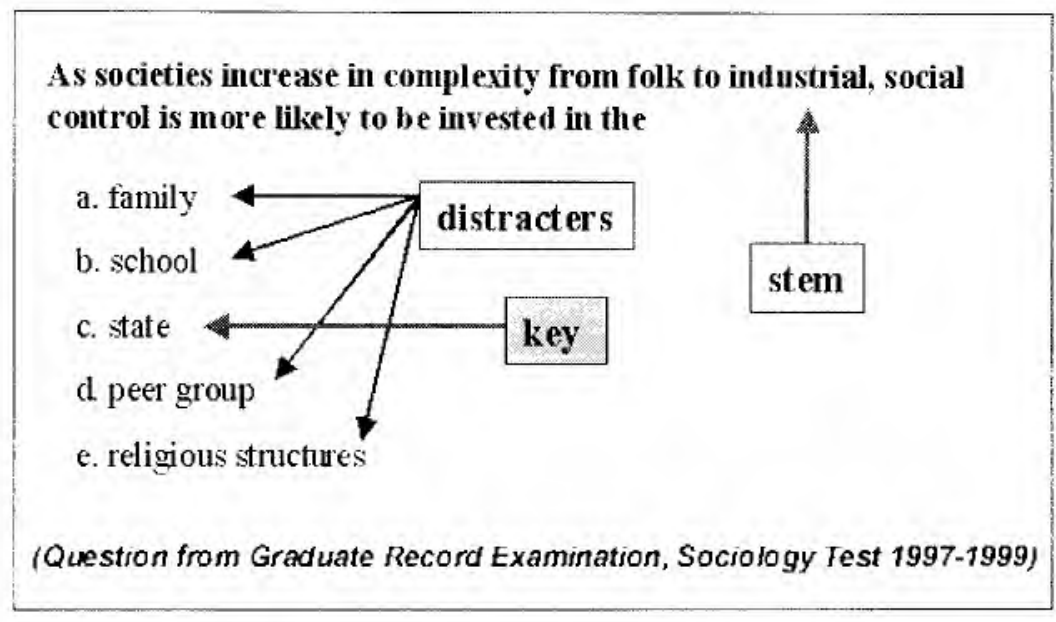

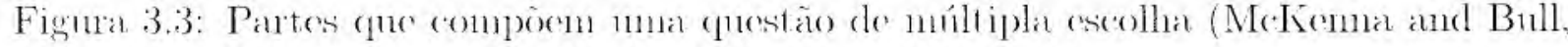
$19999)$

Com base nesta forma geral. sào estabelecidos tipos de questões que podem ser

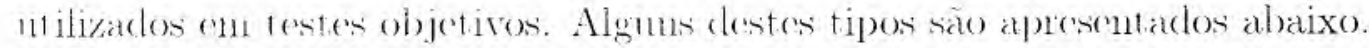

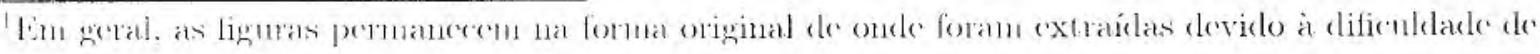

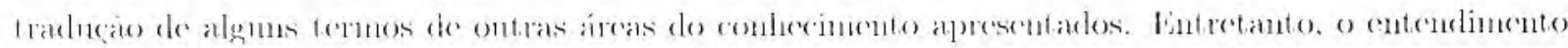
das mesmas nato comprometido.
} 


\subsection{Tipos de questões}

Eina boa maneira de avaliar o conherimento de habilidades intelectnais dos estudantes é por meio das formas tradieionais de avaliação. on seja. respostas discmusivas. Apesar de permitir que o estudante demonstre nunat série destas habilidade's. ela não possui muita

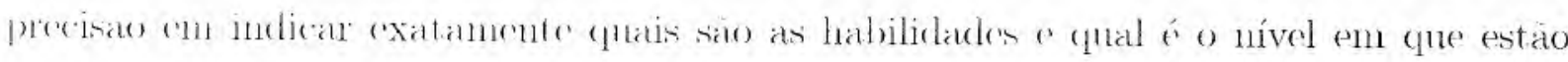
scuclo avaliadas (Brown ot al.. 1999)). Respostas a algumas destas questós podem ser

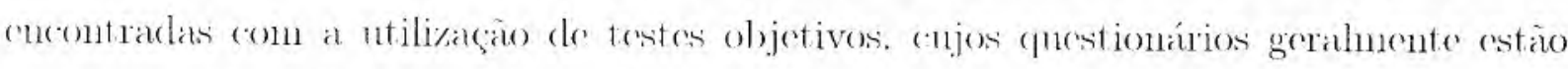

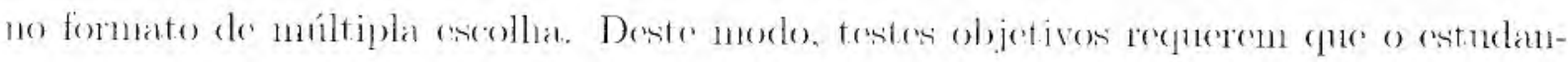

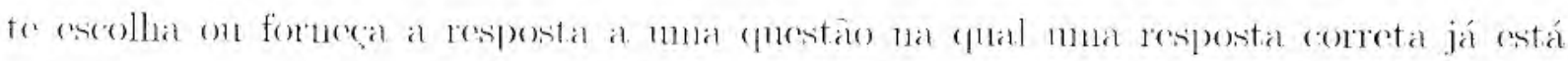

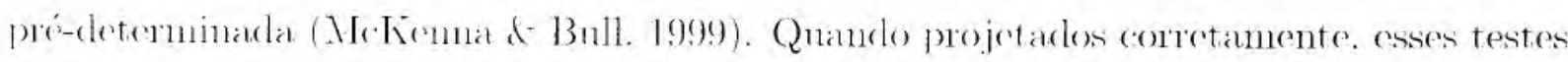

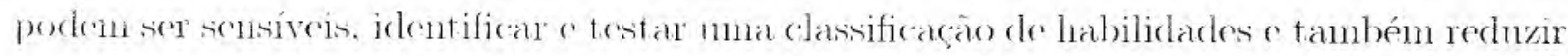
a possibilidade do estudante obter sucesso por acaso e náo como reflexo do sen conhecimento. Brown et al. (1999). fornece guidelines para anxiliar a produçào de questões de testers objetivos eficazes. tais como:

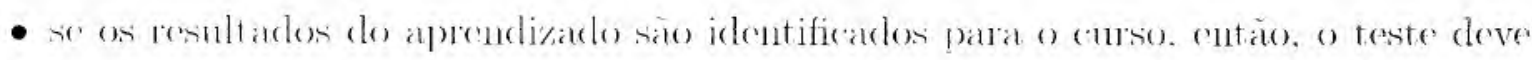
atacioles:

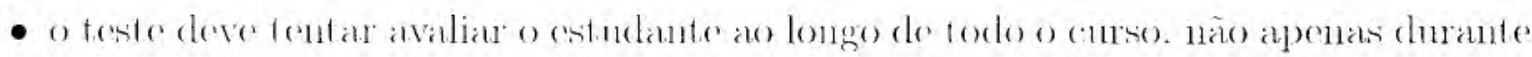
partes dele:

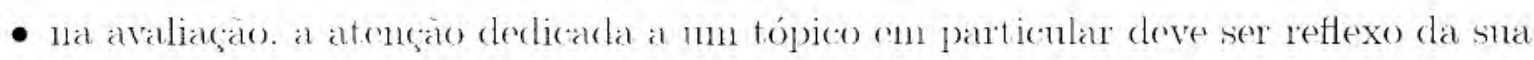

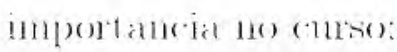

- a avaliaçào deve ser integrada dentro do projeto do curso. e năo tratada à parte e posteriornente.

\section{Múltipla Escolha}

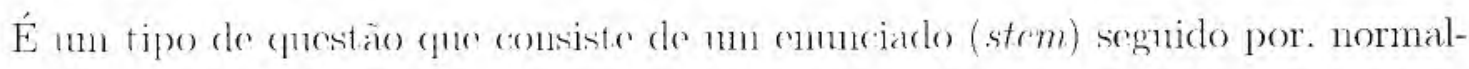

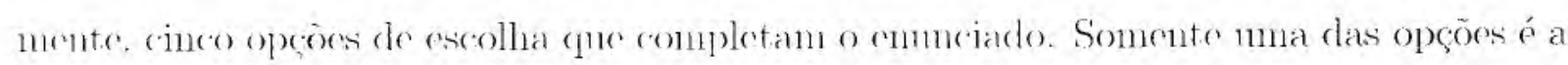
respostal correta. ort a melhor resposta. e o restante são distratores. O papel do estudante é escolleer a partir das opcóes de resposta. aquela que elo considera como correta. Para a 


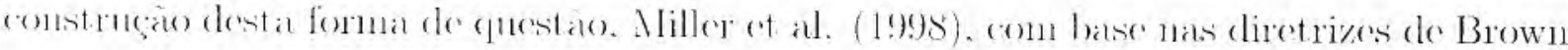

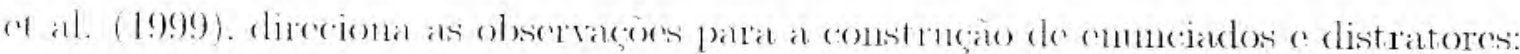

1. Construindo o enunciado:

- Apresentar una afirmação única e definitiva para ser completada ou respondida a partir das várias escolhas.

A Fignara 3.4 mostra 110 excmplo A que o enumciarlo nào é claro o suficiente para identificar o que a duestao está perginutando. Já o exemplo B identifica a questão

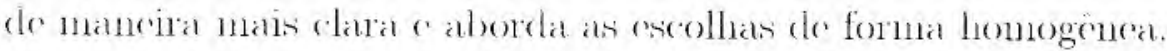

A. Weak question:

\section{Polysaccharides}

a. are made up of thousands of smaller units called monosaccharides

b. are NOT found in the aloe vera leaf

c. are created during photosynthesis

d. can be described by the chemical formula: $\mathrm{CHHOH}$
B. Improved question:

Polysaccharides of the plant cell wall are synthesized mainly in
a. the endoplasmic reticulum
b. the cytosol
c. the plasma membrane
d. the Golgi complex
e. amyloplasts

Figura 3.4: Qunestao aperfeicoacla do GRE - Teste de Biologia Molecular o Celular. 19971999 (Mr hiemat and Bull. 1999).

- Evitar material desnecessário e irrelevante no enunciado.

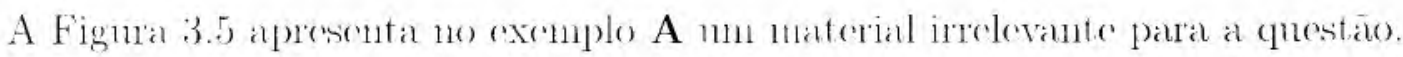

- Utilizar linguagem clara e simples no enunciado.

Linguagens complexas tendem a direcionar o teste mais para a compreensão da Leitura do fure para avaliar se o estudande conhece a matéria. A Figura 3.6 traz um exrmplo desise latos.

- Utilizar negações nos enunciados de forma moderada e de preferência destacá-las.

A Figma 3.7 exemplifica estat observacios. 


\begin{tabular}{|ll|}
\hline A. Weak question: & B. improved question: \\
$\begin{array}{l}\text { Paul Muldoon, an Irish postmodern poet } \\
\text { who uses experimental and playful }\end{array}$ & $\begin{array}{l}\text { Paul Muldoon uses which poetic genre in } \\
\text { "Why Brownlee Left"? }\end{array}$ \\
\begin{tabular}{ll} 
language, uses which poetic genre in & a. sonnet \\
"Why Brownlee Left"? & b. elegy \\
a. sonnet & c. narrative poem \\
b. elegy & d. dramatic monologue \\
c. narrative poem & e. haiku \\
d. dramatic monologue & \\
e. haiku & \\
\hline
\end{tabular}
\end{tabular}

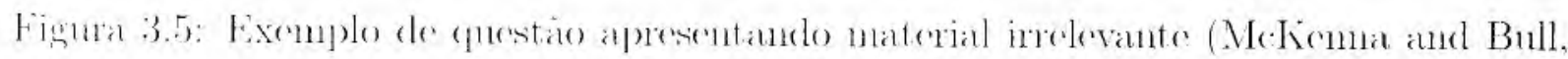
$19999)$.

- Adicionar mais informações no enunciado ao invés de duplicá-las nas opções de resposta.

Esta ocomeneia pode sen vista na Fignim 3.8.

2. Escrevendo distratores:

- Assegurar que dentre as respostas de uma questão tradicional de teste de múltipla escolha haja somente uma resposta correta.

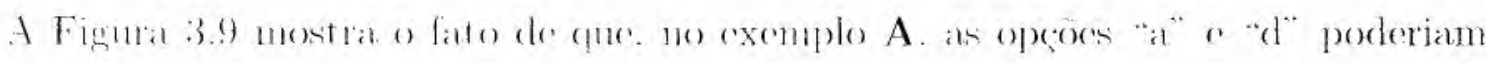

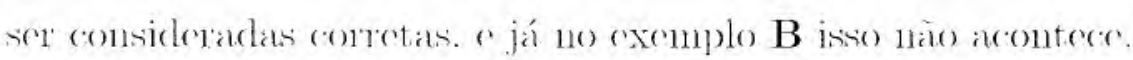

- Usar somente alternativas plausíveis e relevantes nos distratores.

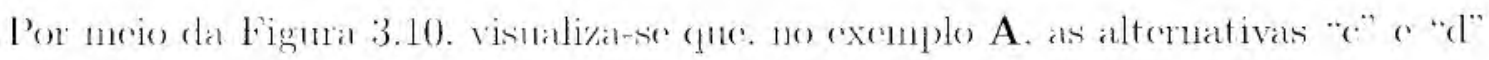
nive sate distratores importantes.

- Evitar pistas das respostas corretas.

Na Figma 3.11. no exemplo A. o nco do artigo "an" sugere a resposta correta, visto (que enu ingles elé ó utilizado antes de palavras que sào iniciadas con vogal. Já na questao B. a finalizaçáo com "a/an" evita clar pistas para a resposta.

- Se possível, evitar escolhas do tipo "Todas as anteriores" e "Nenhuma das anteriores". 


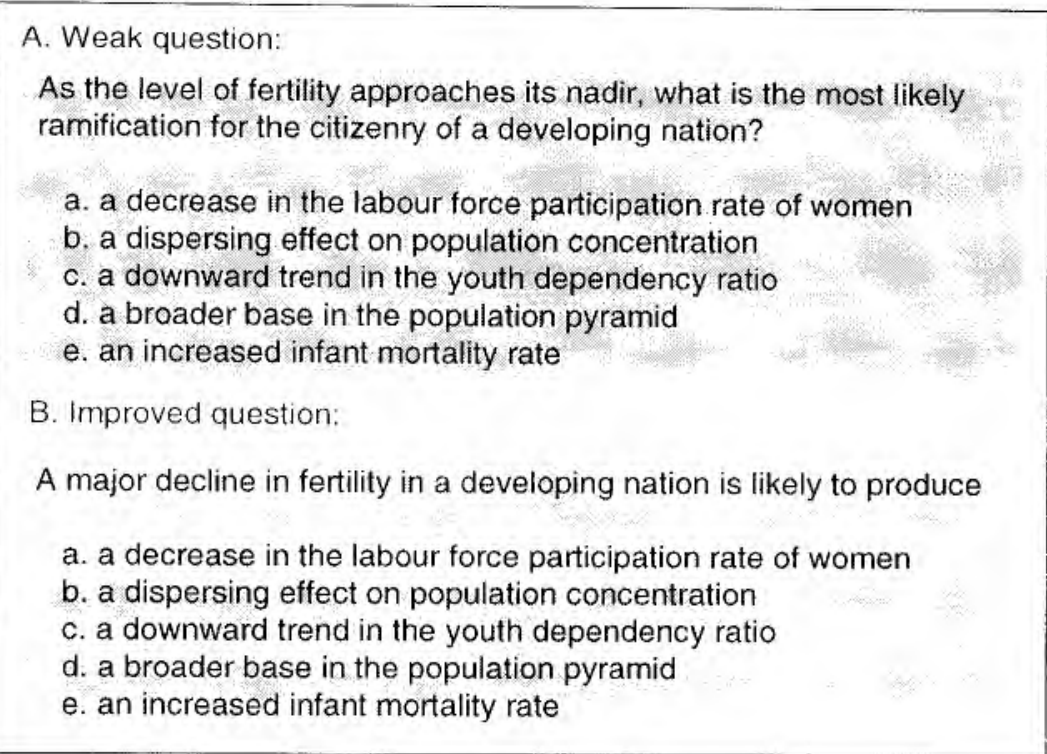

Figmra 3.6: Qnestaio ajerfeicoada do GRE - Teste de Sociologia. 1997-1999 (McKenna and Bull. 19999).

- Obter distratores baseados nos erros comuns dos estudantes ou mal entendidos.

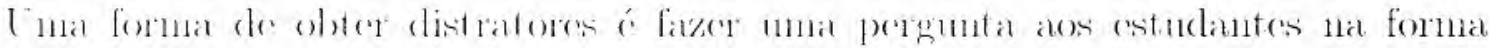

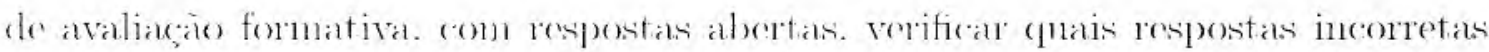

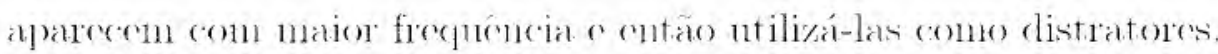

- Não criar distratores que escondam, de maneira confusa, a resposta correta, o que pode ocasionar incerteza em demasia no estudante que conhece a resposta para a questão.

- Fornecer um número suficiente de distratores.

Une grande munero de distratores diminui a probabilidade do estudante escollere a

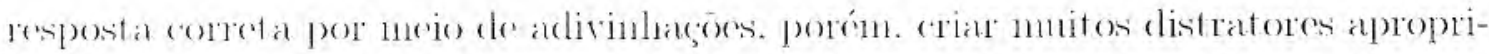

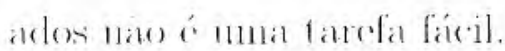

\section{Resposta Múltipla}

É $u m$ tipo especial de questäo de múltipla escollat cujo diferencial está na possibi-

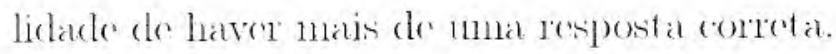




\section{A. Weak question: \\ Which of the following is not a symptom of osteoporosis?}
a. decreased bone density
b. frequent bone fractures
c. raised body temperature
d. lower back pain

B. Improved question:

Which of the following is a symptom of osteoporosis?
a. decreased bone density
b. raised body temperature
c. hair loss
d. painful joints

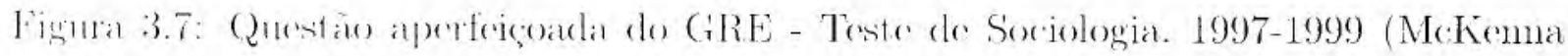
and Bull. 19999).

\section{Verdadeiro/Falso}

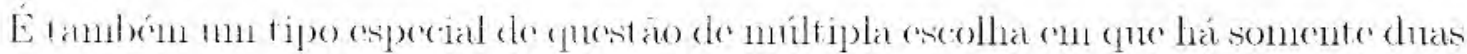

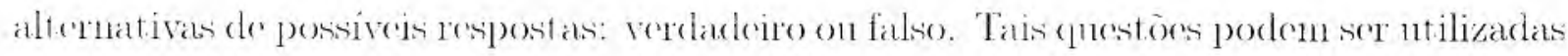
quando se deseja medir a habilidade do estudante ene jelentificar se os emunciados dados

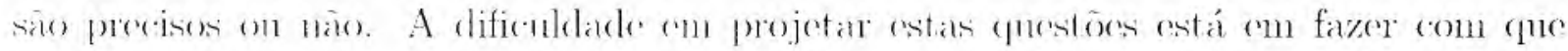

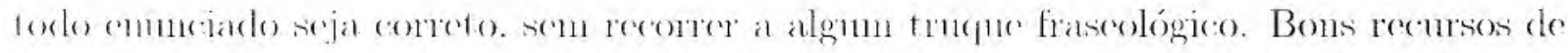

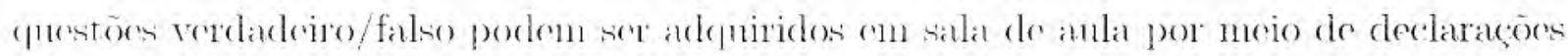
verbatis on escritas por estudantes a respeito de perguntas fritas pelo professor (Miller ot al. 1998). Questoes deste tipo possuem limitacoes tais como:

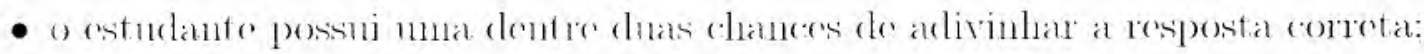

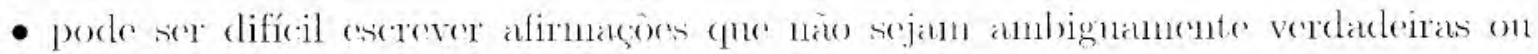
falsals. particularuente parat mu nuaterial eomplexo:

- o formato náo discrimina tão beno o estudante de habilidades diferentes, como fazem ontros tipos do questoes.

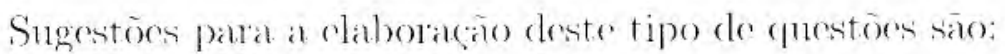

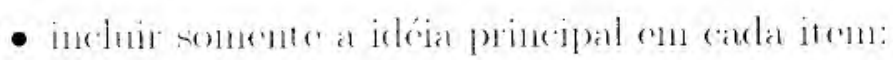

- ntilizar negativas moderadanente. la mesma forma cue em questós de múltipla escollat: 


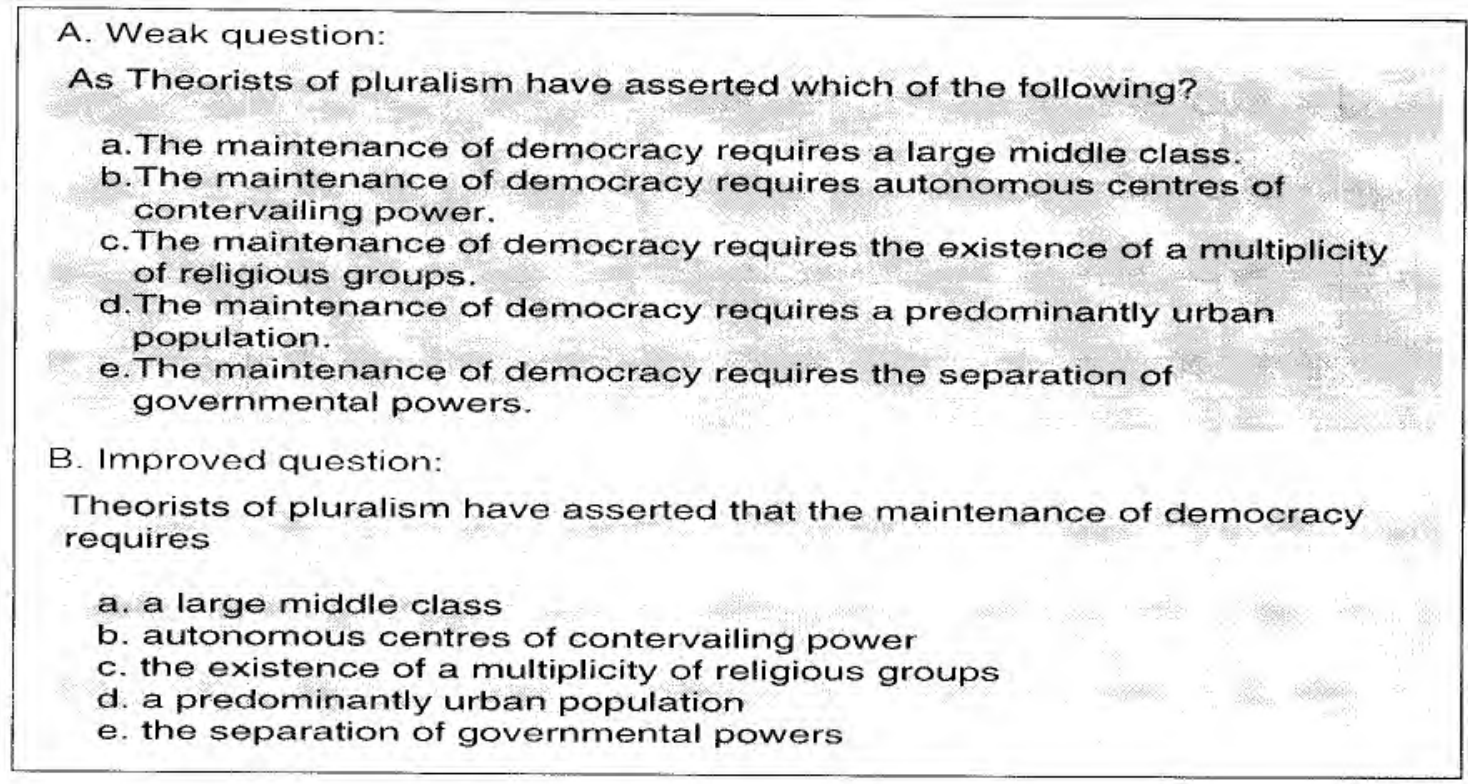

Figura 3.8: Questónes do livro de teste GRE Sociologia. 1997-1999 (MeKema and Bull. $19999)$

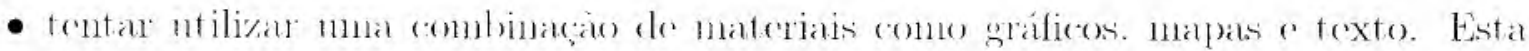
combinaceio permite obter resultades do aprendizato mats avancados:

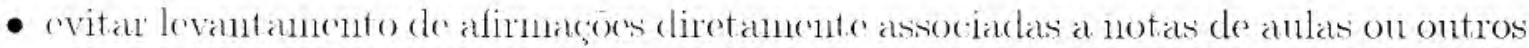
materiatis do (1urso:

- evitar o nese de palavras cque indiguem a resposta correta. Termos absolutos como

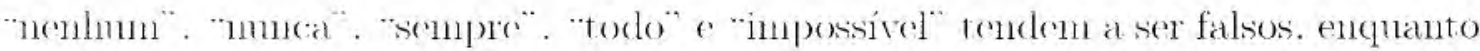

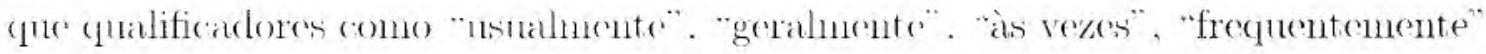
săo provavelnente verdiuleiros.

\section{Completar}

Neste tipo do questào. o cistudante responde à questão completando uma sentemcar de preferencia utilizando mua on duas palavras. Na forma simplificada. a sentença

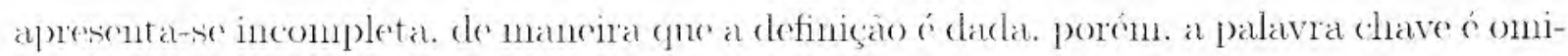
ticla. Há também a possibilidade do ntilizar diagramass on gráficos. nos quais os rótnlos

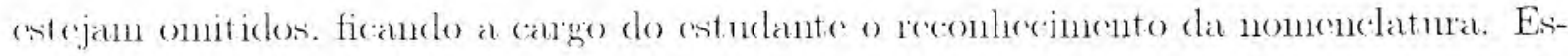

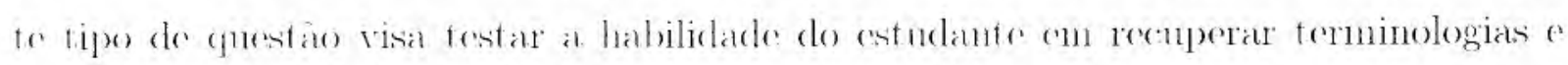




\begin{tabular}{|ll|}
\hline $\begin{array}{l}\text { A. Weak question: } \\
\text { Which of the following texts is considered } \\
\text { to represent the pinnacle of } \\
\text { modernist achievement? }\end{array}$ & $\begin{array}{l}\text { B. Improved question: } \\
\text { Which of the following texts represents } \\
\text { one of the high points of modernist } \\
\text { achievement? }\end{array}$ \\
\begin{tabular}{ll} 
a. The Waste Land & a. The Waste Land \\
b. Middlemarch & b. Middlemarch \\
c. "Ode to a Nightingale" & c. "Ode to a Nightingale" \\
d. Ulysses & d. Ethan Frome \\
e. Ethan Frome & e. "My Last Duchess" \\
\hline
\end{tabular}
\end{tabular}

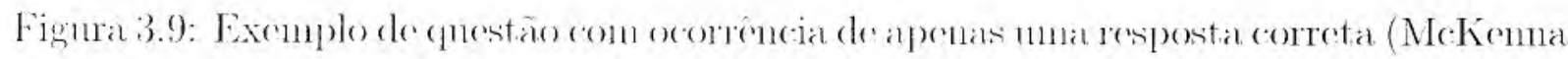
and Bull. 19999).

\begin{tabular}{|ll|}
\hline $\begin{array}{l}\text { A. Weak question: } \\
\text { Dichotic presentation of stimuli and } \\
\text { shadowing are often used in the study of }\end{array}$ & $\begin{array}{l}\text { B. Improved question: } \\
\text { Dichotic presentation of stimuli and } \\
\text { shadowing are often used in the study of }\end{array}$ \\
\begin{tabular}{ll} 
a. brightness constancy & a. brightness constancy \\
b. sensory scaling & b. sensory scaling \\
c. shadow dancing & c. illusions \\
d. cartoons & d. depth perception \\
e. selection attention & e. selection attention \\
\hline
\end{tabular} \\
\hline
\end{tabular}

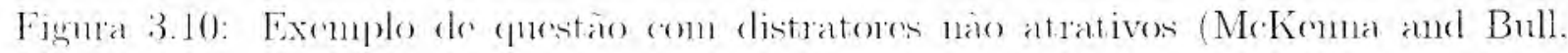
$1999)$.

reconleere o contexto dentro de temas particulares que estato sendo ntilizados.

\section{Completar Múltiplo}

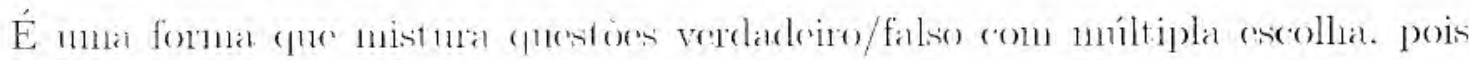

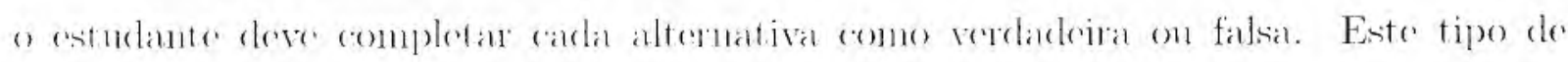

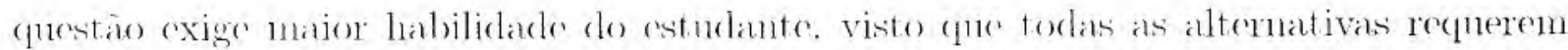

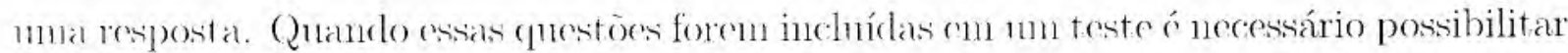

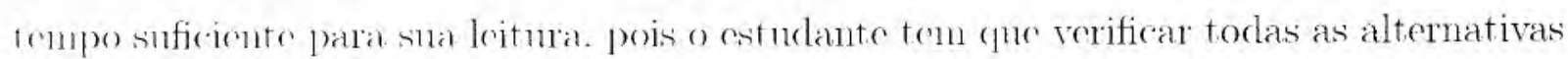
dardas. () exemplo da Tabela 3.1 da Eniversidade de Lancantere por Mathews. 1977 apud (Miller et al. 1998). ilustra este tipo de (puestão: 


\begin{tabular}{|ll|}
\hline $\begin{array}{l}\text { A. Weak question: } \\
\text { A fertile area in the desert in which the } \\
\text { water table reaches the ground surface is } \\
\text { called an }\end{array}$ & $\begin{array}{l}\text { B. Improved question: } \\
\text { A fertile area in the desert in which the } \\
\text { water table reaches the ground surface is } \\
\text { called a/an }\end{array}$ \\
$\begin{array}{ll}\text { a. mirage } & \text { a. mirage } \\
\text { b. oasis } & \text { b. oasis } \\
\text { c. water hole } & \text { c. water hole } \\
\text { d. polder } & \text { d. polder }\end{array}$ \\
\hline
\end{tabular}

Fingua 3.11: Exemple de (questate com pista da resposta corteta (Mr.Koma and Bull. $19999)$.

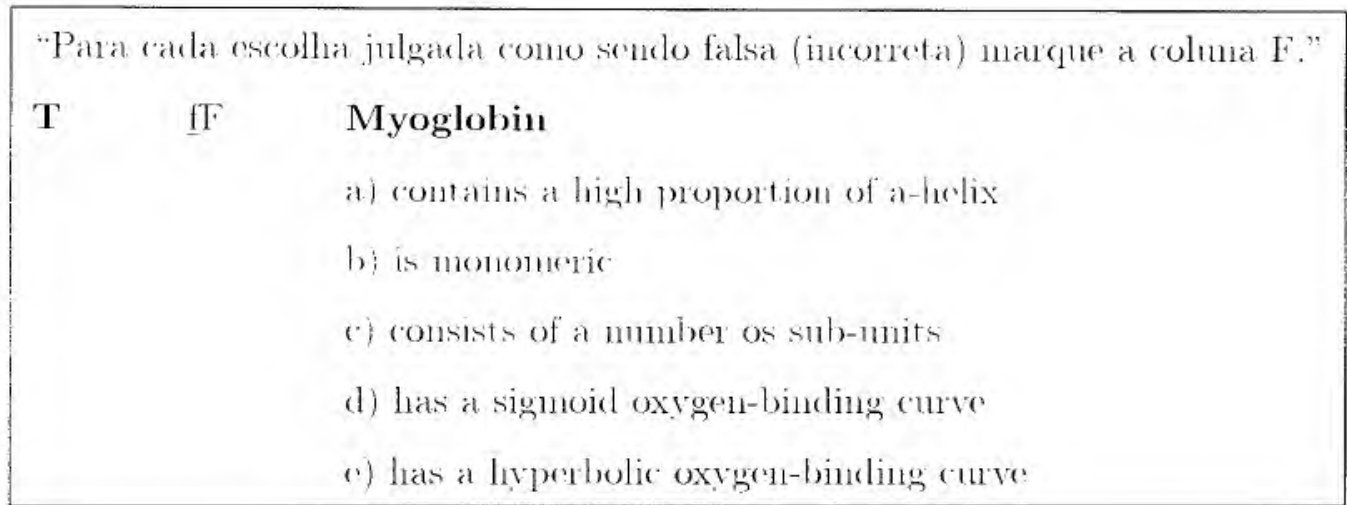

Taleda 3.1: Exomplo do tipo de cunestáo de Complotar Múltiplo (Miller-98).

\section{Combinação/Correspondência}

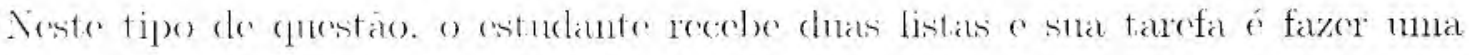

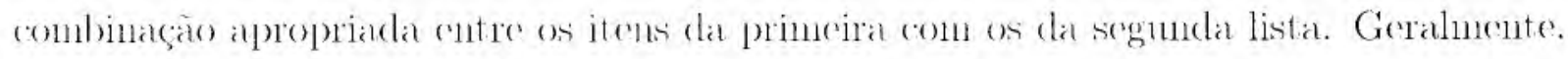

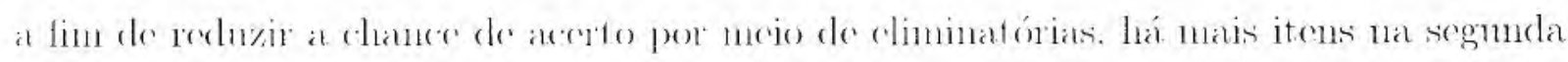

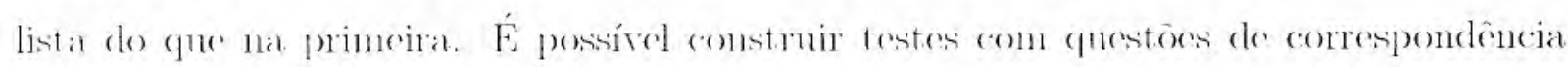
com objetivo de obter medidas de comberimento dos níveris majs elevados da hierarquia de (onherimento. mas ó mais commun curentrá-los com a finalidade de testar a capacidade do estudante enu recouperar o recouleceer un assiunto da matéria. A Figura 3.12 traz $1 \mathrm{~mm}$ excmplo desta forma de questrono.

\section{Análise de Relação/Causa-Efeito}

Neste tipo de questato ó testade o conhecinento concreto do estudante e sua habili-

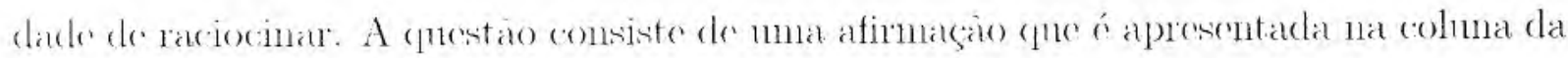


Directions: Column I contains descriptions of geographic characteristics of wind belts. For each statement find the appropriate wind belt in Column II. Record your answer in the appropriate space on the answer sheet. Answers may be used more than once.

\section{Column I}

1. Region of high pressure, calm, and light winds

2. The belt of calm air nearest the equator

3. A wind belt in the northern hemisphere typified by a continual drying wind

4. Most of the United States is found in this belt

\section{Column II}
a. Doldrums
b. Horse latitudes
c. Polar easterlies
d. Prevailing easterlies
e. Prevailing westerlies

Figura 3.12: Excmplo do tipo de (1uestano de Combinatuâ/Correspondência (McKenna and Bull. 1999).

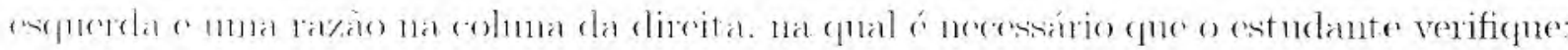

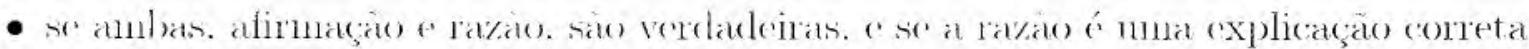
(lat afirmacicio:

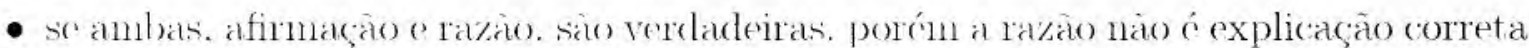
dat afirmatciate:

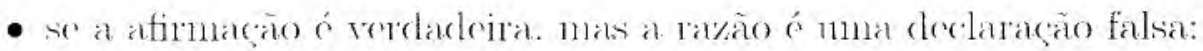

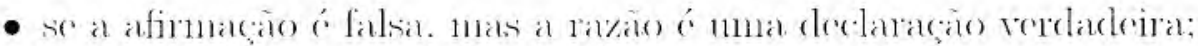

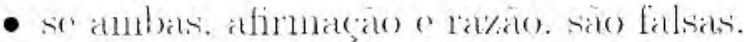

A Tabela 3.2 apresenta unu exemplo deste tipo de curestäo.

A construcăo de bons testes objefivos também recuner a avaliação de cada questão criarla, antes de serem ntilizarlas pelos estudantes. Mathews. 1997 apud (Miller et al.. 1998). propor a segunite rhestimst para ser verificada antes de adninistrar o teste para o estudiante: 


\begin{tabular}{|c|c|c|}
\hline Assertion & & Reason \\
\hline $\begin{array}{l}\text { The blood sitgar level } \\
\text { falle pereipitonsty after } \\
\text { leppatectome }\end{array}$ & Beratise & $\begin{array}{l}\text { The slycogen of the } \\
\text { liver is the principal } \\
\text { semere of blood sugar }\end{array}$ \\
\hline
\end{tabular}

Labela 3.2: Lxemplo de questào do tipo Análise de Rolacáo/Cansa-Efeito (Miller-98).

1. Ler a responder as question:

(a) Existe malis de mona respostat cometal?

(b) Existe algmuar ambigniclade na questaon on na(s) resposta(s)?

(6) A questao porte ser lida e resipondida no tempo determinado para avaliação?

2. Verificar o contureiado:

(a) A questano á importanteo suficiente para que ela seja respondida?

(b) Há infomacào suficiente fornecida no munciado para respondé-la?

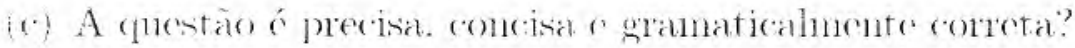

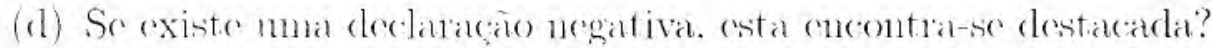

3. Verilion as respostas:

(a) Todas são plansivecis?

(b) A resposta correta contenu alguma pista?

(c) Toclas as respostas possuen o mesmo tamanho?

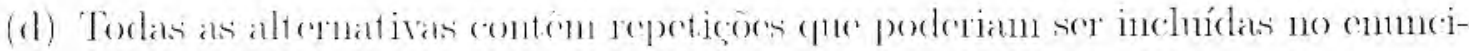

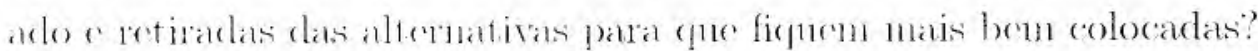

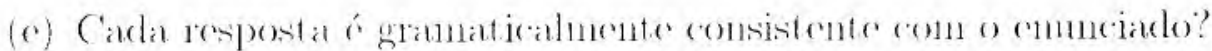

1. Verificar of formato:

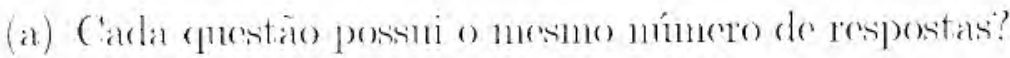




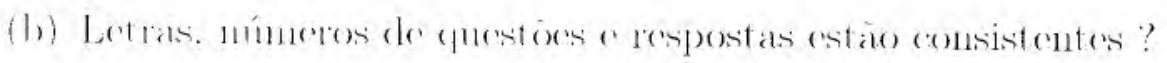

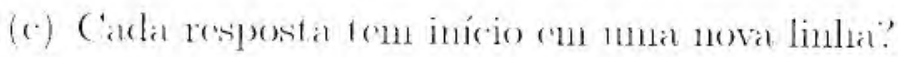

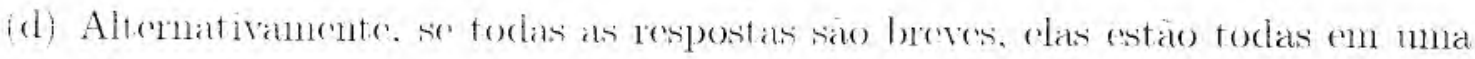
linha:

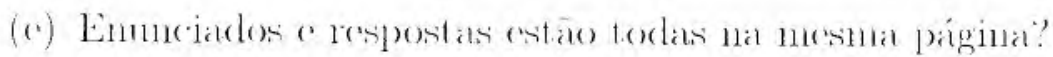

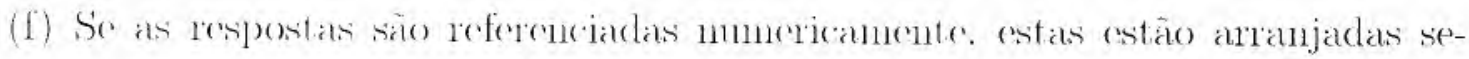

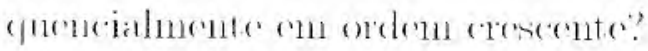

5. Clansificar as questós:

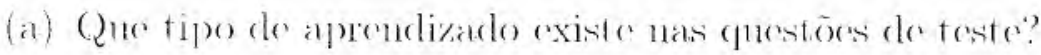

(b) A cure área da matéria pertener o teste?

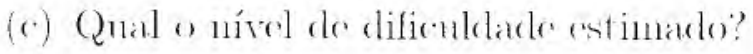

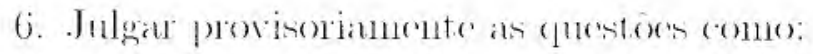

(a) Accitatrel.

(1) Aceitível com algumas peguenas alteracoes.

(c) Reconstrucào das necessiclades principais.

(d) Rejeitável.

\subsection{Abordagens para a Avaliação Informatizada}

Conforme visto mas secoes anteriores. existem diveriats maneinas do computador estar en-

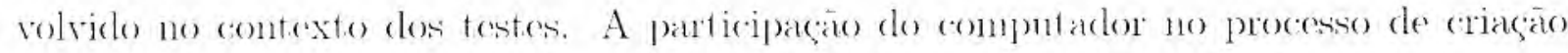

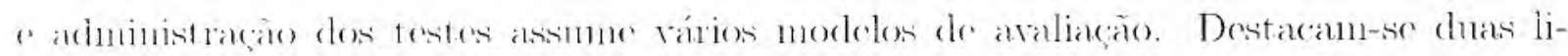

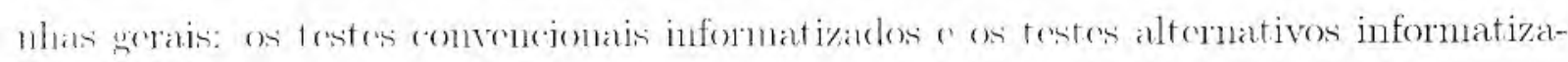

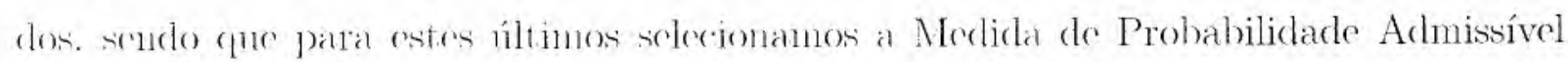
(AIPA)(Shuford \& Brown. 1974). que (mvolve testes de múltipla escolha cujas respostas

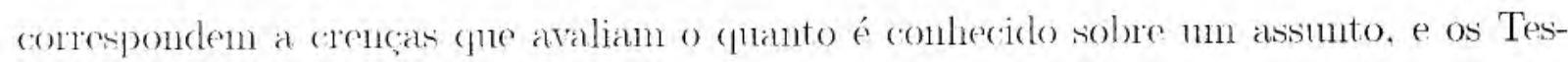
tes Adaptativos (O)lea of al. 1999). (1ue adaptant ats questioes de mu teste ao nível de bathilidade de cada entudante. 


\subsubsection{Métodos Convencionais}

Na abordagem convencional. o número de questoes dos testes ó o mesmo para todos on participantes. fodos os itens tom a mesma difienldade " sào apresentados na mesma

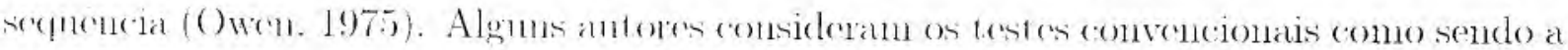

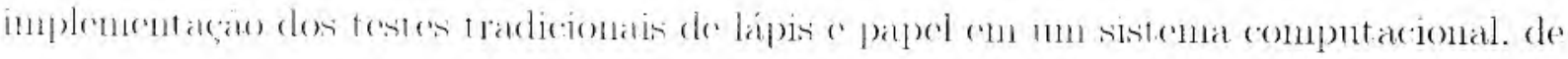

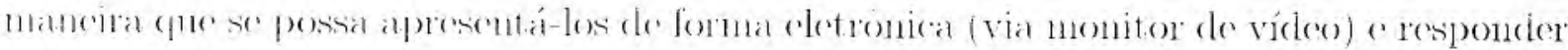

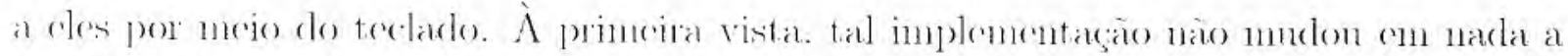

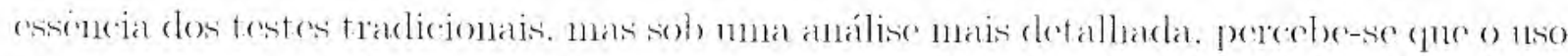

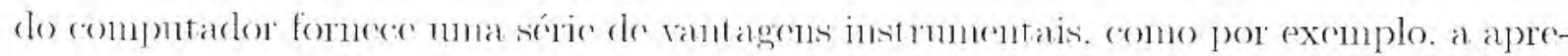

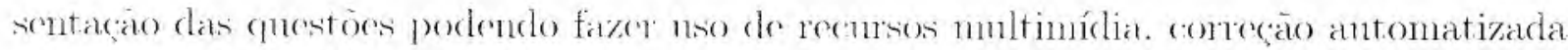
das respostas. possibilidarde de computar cálenlos estatísticos e rapiciez na divulgação dos irsultados.

En linhas gerais. o nso do computador na aplicagao dos testes convencionais permite a ntilizaçare de guestós de teste mais complexas " mais próximas da realidade.

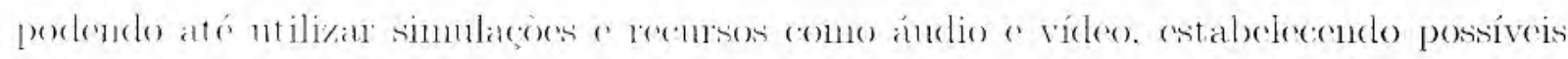

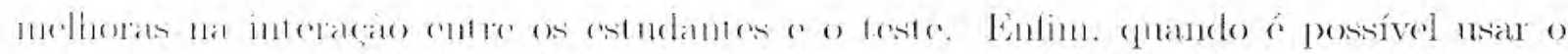

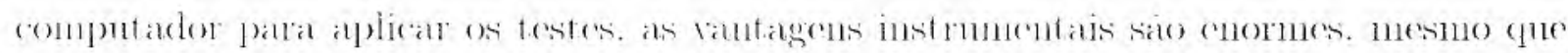

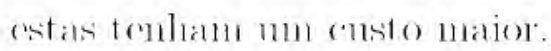

Näo há dúvidas que existem várias vautagenes operacionais no no do computador na aplieacäo de testes tradieionais. Nense cenário. de convivencid entre os testes conven(ionais informatizados e o formato dre lápis e papel. a duivicla que surge é se os resultados de ambas aplicacoes sâ estritamente os nesmos. on se a mudança introduzida pelo nso do computador altera as propriedader psicométricas don trestes.

A priori. tem-se a impressato de que a linica mudanca radical nos testes, dado a sua

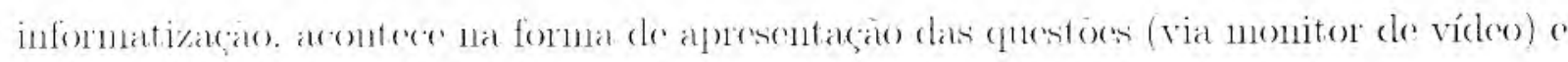

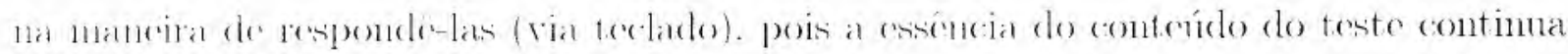

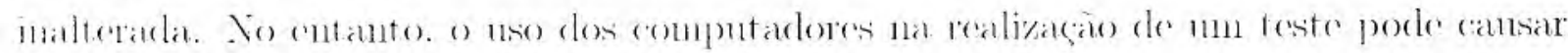
diforentes inpactos nos astudauter.

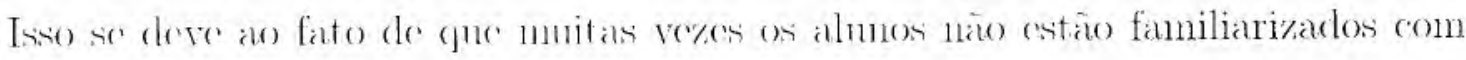

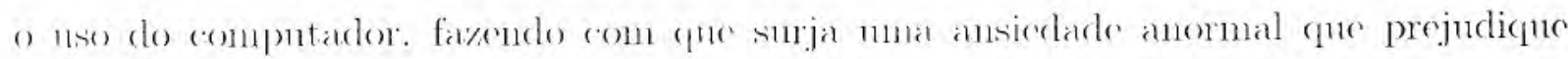




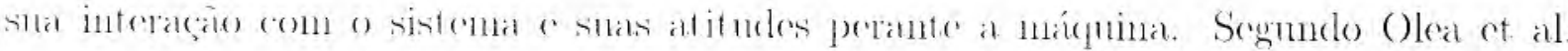

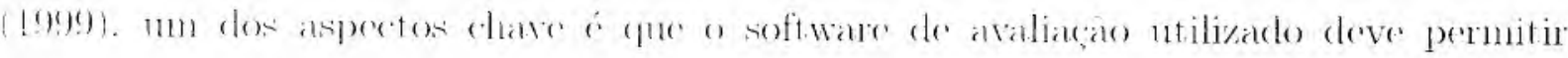

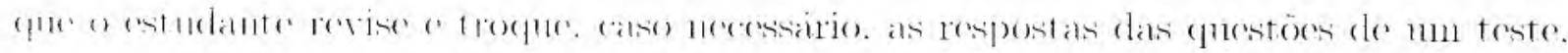

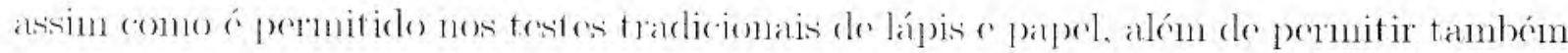

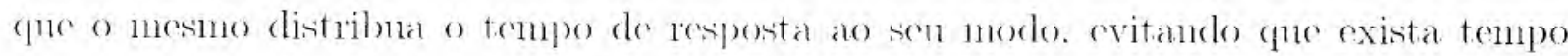

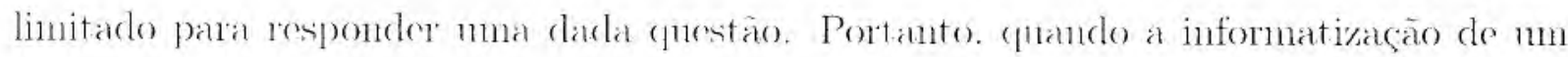

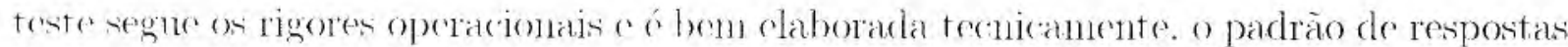

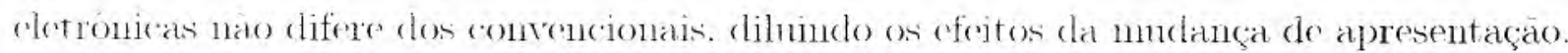

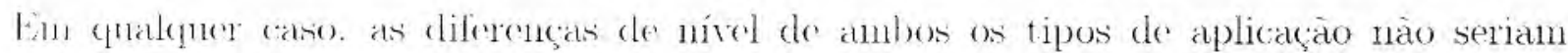
Imu problema insolúvel. poin na atnalidarle existe teconologia eficiente para equiparar as

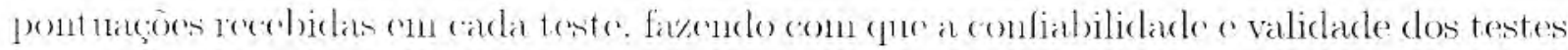
sejantre expivialentes.

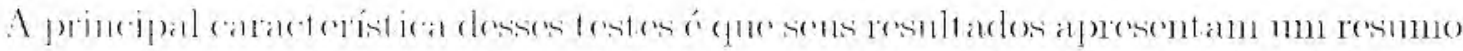

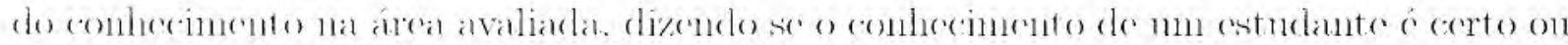

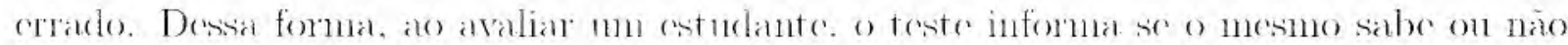

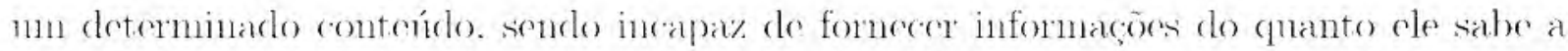
resperito do couteído.

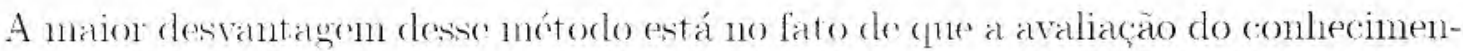
to realizarla per ele a sereranente distorecila e tenelenciosa. pois existe ainda uma grande

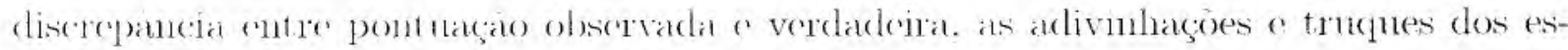

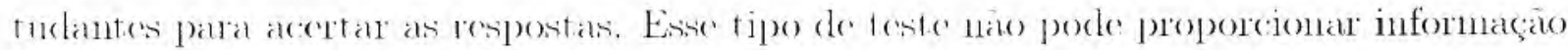

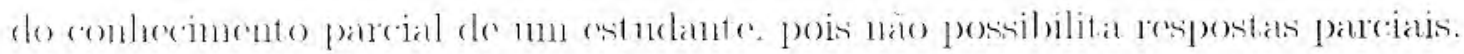

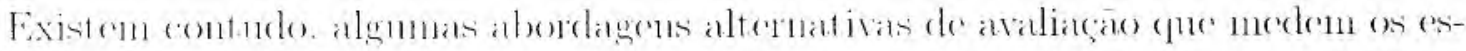

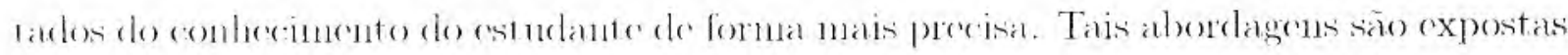
lat proxintat sulnseço.

\subsubsection{Métodos Alternativos}

\section{Testes baseados na Medida de Probabilidade Admissível}

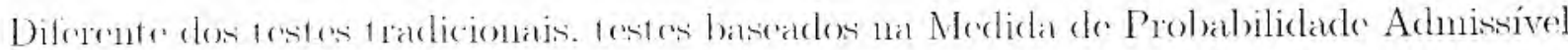
f Shluforel d Brown (1974). Bruno (1986). Brumo (1987). Brino (1988). Klinger (1997)) 


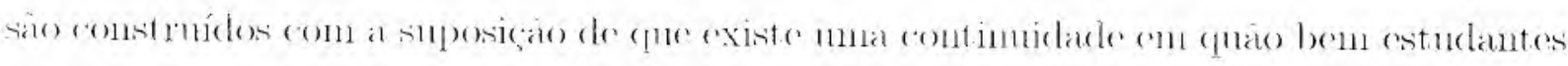

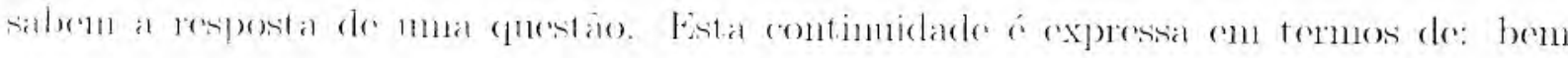

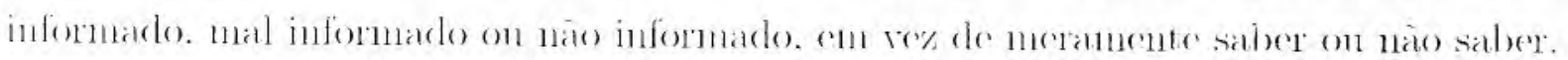

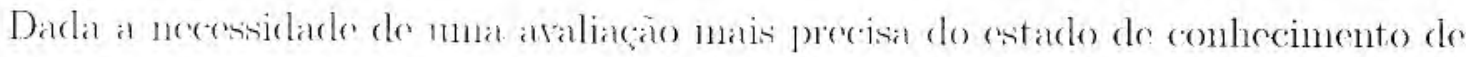
$11 m$ estudanter a Merlida de Probalsilidarle Admissivel proctura valorizar o conhecimento

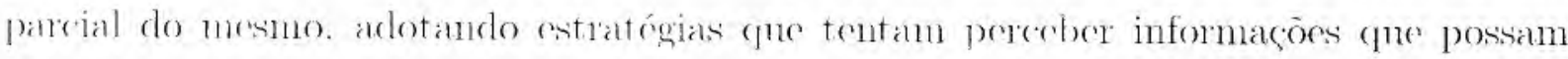

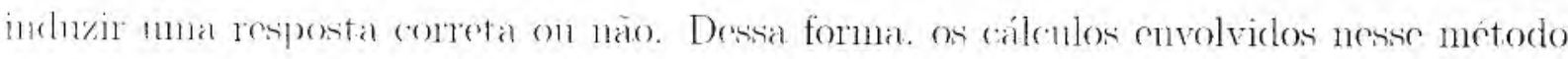
tentann quantificar o conherimento do estuctante de nuodo a ciassificá-io de acordo com o sen nível de informaça real, seja cle lotal on parcial. sendo mas classes divididas em: Totalnente Informado. Quase Informanto. Pareialmente Informado. Desinformado, Mal

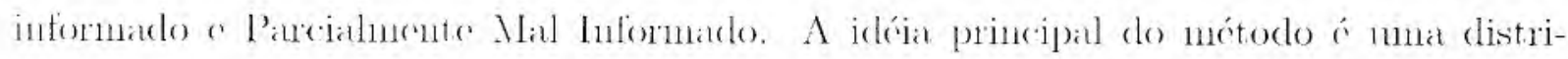

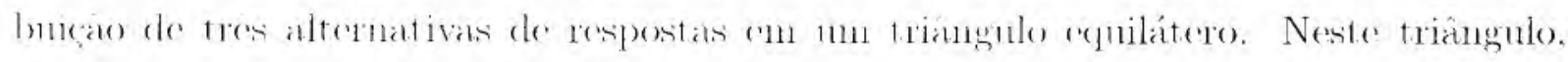
sion localizados pontos adienonato gue se reforem a alternativas adicionais de resposta que

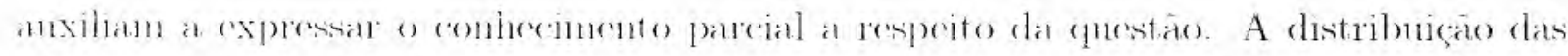
alternativas no triangulo equilítero ó foit a cono mostra a Fignura 3.13. parte a).

Com base nesta distribuicào é aconselhável gue o estudante temha o seguninte comportancento:

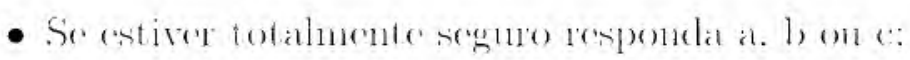

- Se estiver totadmente inseginore responda ma

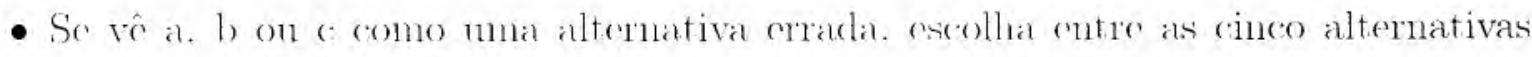
focalizarlas na linha oposta:

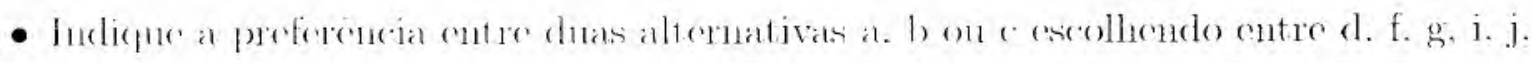
1 .

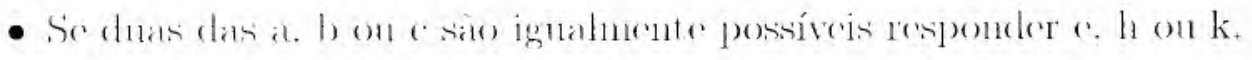

Assim. pelos valores dos pontos. c rantajoso responder a. b on c sonente quando Se está mais do que $90 \%$ certo. Caso contrário, mana resposta 13 tem maior vantagen. As fracoes sato nadas no cálenlo cla pontuaçäo (Figuma 3.13. parte b)) e representan 


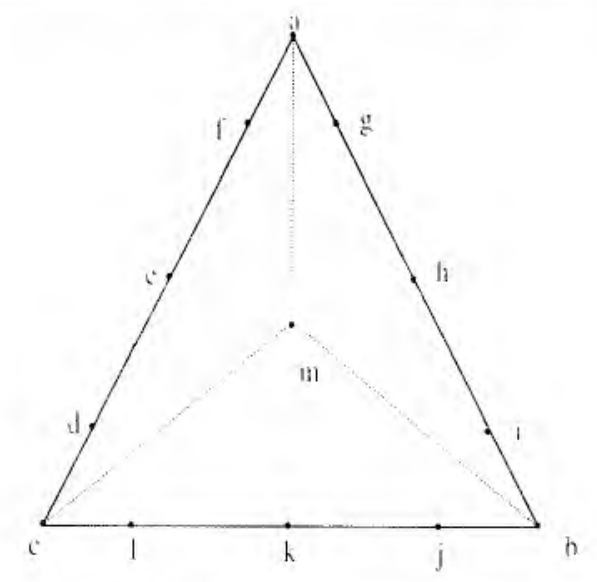

a) Três respostas e trèc alternativas

$\begin{array}{lrrr} & \mathbf{a} & \mathbf{b} & \mathbf{c} \\ \mathbf{a} & 30 & -100 & -100 \\ \text { b } & -100 & 30 & -100 \\ \mathrm{c} & -100 & -100 & 30 \\ \mathbf{d} & -10 & -100 & 20 \\ \mathrm{e} & 10 & -100 & 10 \\ \mathrm{f} & 20 & -100 & -10 \\ \mathrm{~g} & 20 & -10 & -100 \\ \mathrm{~b} & 10 & 10 & -100 \\ \mathrm{i} & -10 & 20 & -100 \\ \mathrm{j} & -100 & 20 & -10 \\ \text { k } & -100 & 10 & 10 \\ \mathrm{i} & -100 & -10 & 20 \\ \mathrm{~m} & 10 & 0 & 0\end{array}$

\begin{tabular}{|c|c|c|c|}
\hline Escolha & Letra & Pontos & Fração \\
\hline Correlia & abc & 30 & 1.000 \\
\hline Erradia & $\ldots$ & .100 & 0.000 \\
\hline Desinformada & $\mathrm{m}$ & 0 & 0.769 \\
\hline Quase corretit & digij| & 20 & 0.923 \\
\hline Entré duas & chk & 10 & 0,840 \\
\hline Quilse errida & dl 1 ij & -10 & (0.692 \\
\hline
\end{tabular}

c) Matriz de Perda

b) Valores de A à M indicando crença

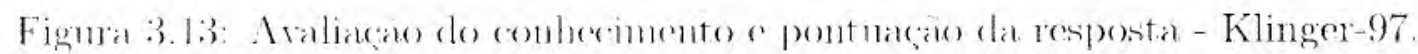

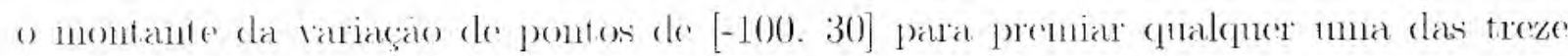

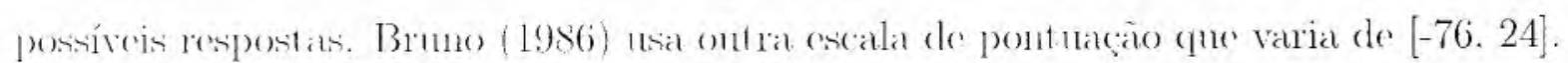

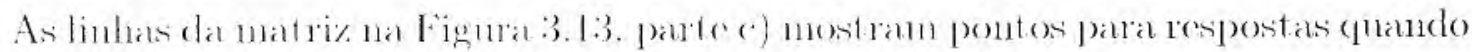
a coluta respertiva formeta.

O método de Medicla de Probabilidade Arhnissived será descrito detalhadamente no Capítulo 5 .

\section{Testes Adaptativos}

Tostes Aclaptativos (TAIs) sầ 1 mu método de teste no qual diferentes conjuntos de questoess são adninistrados para diferentes individuos, dependendo do nível de habilidade do carla 111n. () testes adaptat ivos contrast an com os festes convencionais informatizados

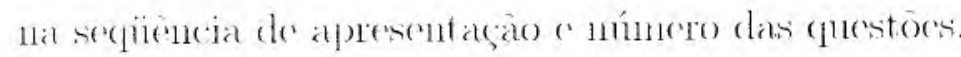




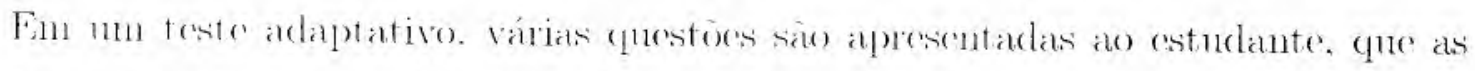

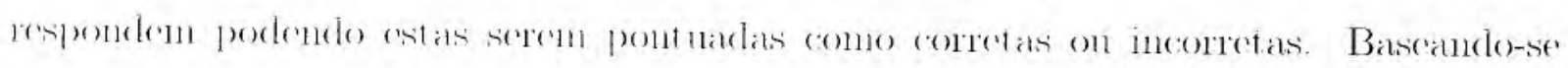

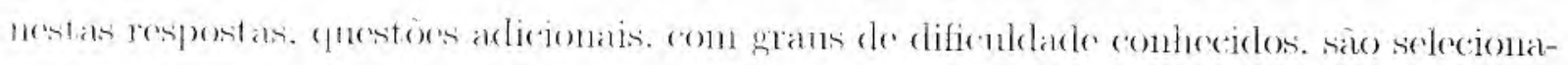

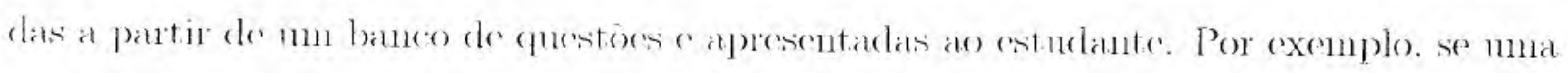

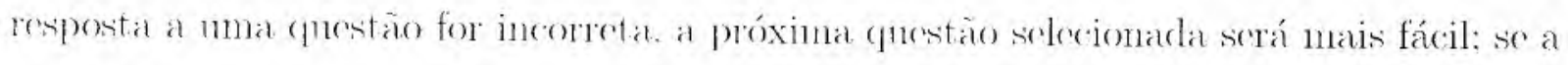

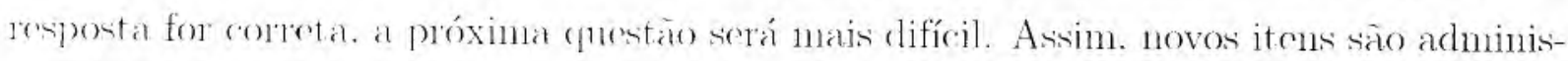
trados e respondiclos. sendo os itens subsecunentes selecionados com base na resposta de rada indivídno.

Existem diferentes abordagens dos testes adaptativos, bascadas principalmente

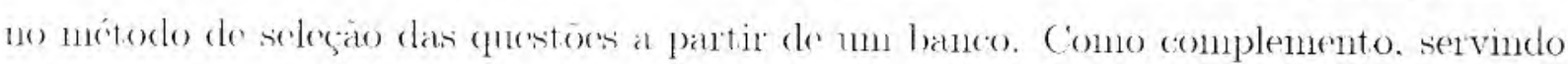
conno carateteristiea essencial do func ionanento dos testes. deve-se considerar a habilidade

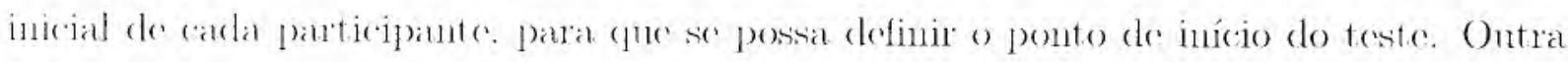

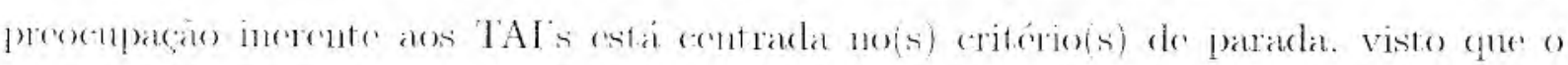

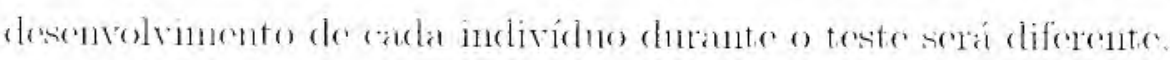

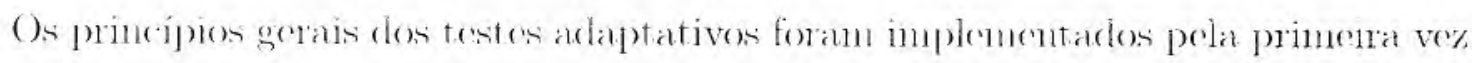

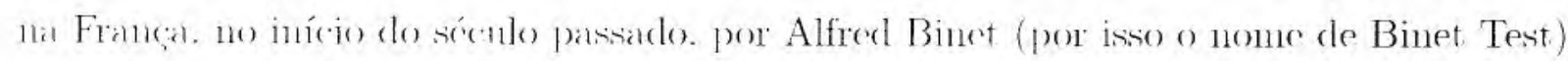
om sen laboratório. e resultaram no que foi chamado Teste do Inteligencia de Binet (Weiss. 1985). A estratégia nsada por Binet foi baseada na idéia do ranificação fixa. usando uma

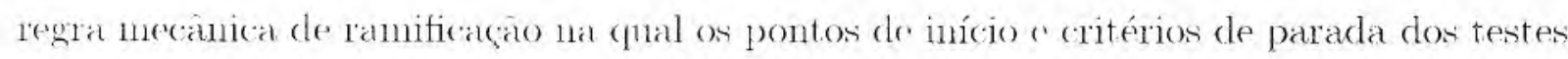
ram varláreis.

() Teste de Inteligencia de Binct foi criade para o diagnostico do nivel de mite-

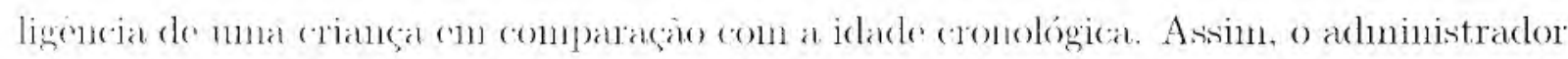

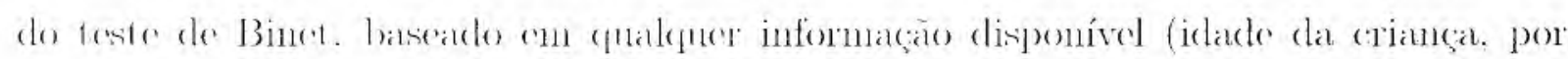

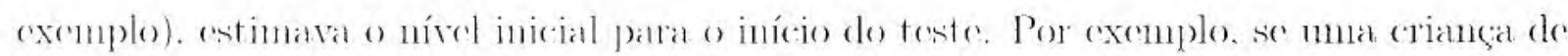

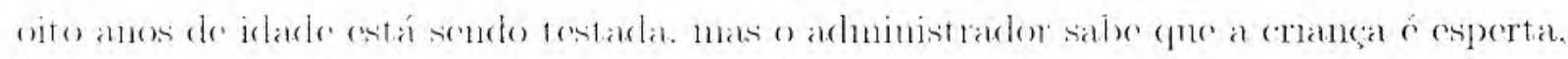

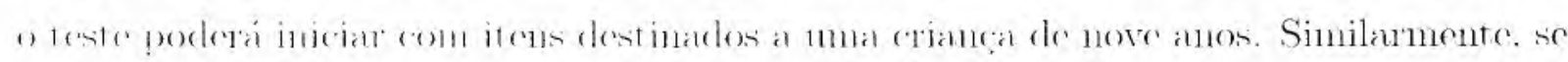
o administrador sabe que a crianca possul certas dificuldades, o teste poderá iniciar com 1101 nivel mais baixo. para numa criancá do sete anos.

Lma vez identificado o nível da criança. os itens correspondentes a tal nível são forneciclos e pontruados secunemeialnente. Depois de todos os itens terem sido administra- 


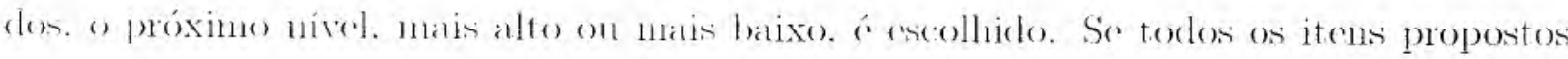

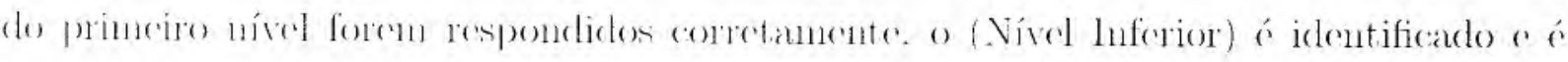

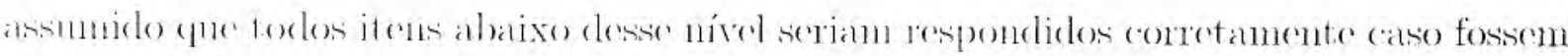

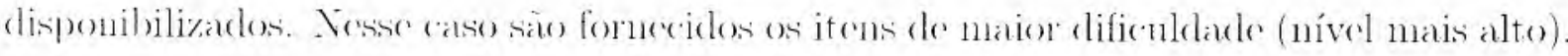

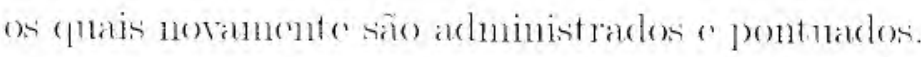

()s itens continuam a ser disponibilizados de acorelo com o ammento do nivel de dificuldade. até quer o (Nível Superior) seja alcancagdo. on seja. até que todos os itens en 1 mu deterninado nível sejann respondidos incorretanente. Q Quando esse nível é iden-

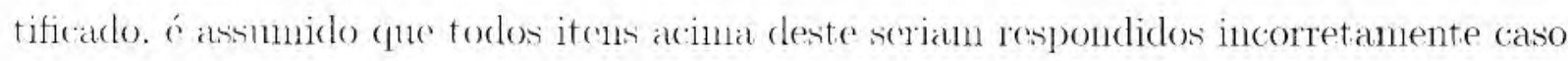

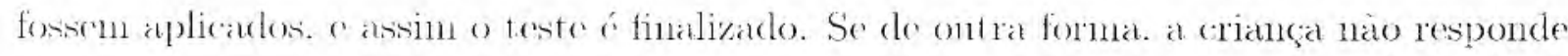

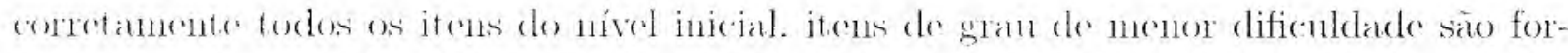

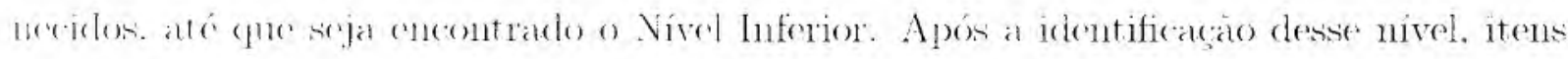

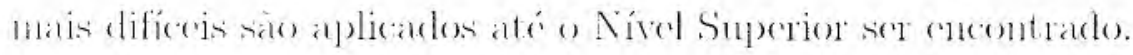

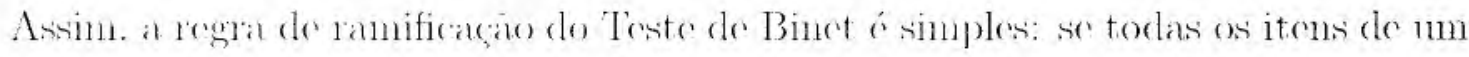

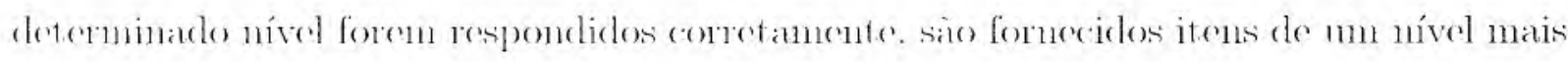

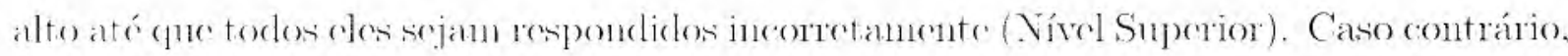

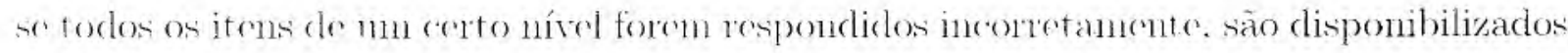
itous de $11 m$ nivel mais baixo até que todos eles sejan respondidos corretamente (Nível Interior).

O) critério de parada do Teste de Binet ocome quando o Nível Inferior e Nível

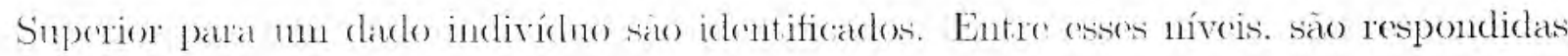

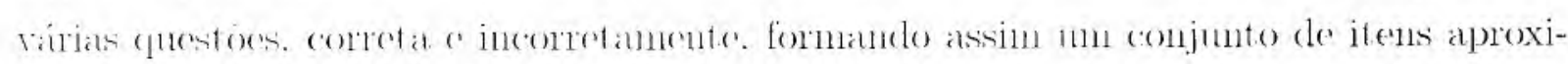

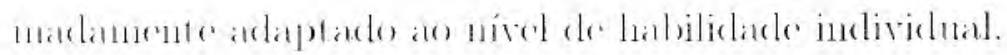

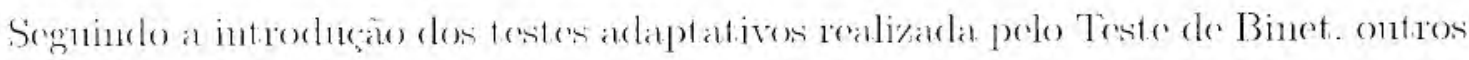
excmplos sugirann apenas no final dos anos 50). Tais testes foram experinentais e usados aperass para estuclate as diversas caractorísticas dos testes adaptativos (Weiss. 1985): Testes Adaptativos de dois astágios ( Two-Stage Adaptative Trosting ). Testes Adaptativos Piramidais ( Pyramidal Aclaptive Test). Testes Aclaptativos Estratificados ( Stratified Adaptative Test ) Testes Adaptativos baseados na Teoria de Resposta de Itens (TRI). 


\section{Um exemplo da aplicação de Teste Adaptativo: GRE CAT}

O) Graduate Rerord Examinatıon (GRE) ó 1ma araliação criada c administrada pelo Educational Testing Service: (ETS). () GRE é concrhido para proporcionar a escolas de pós-graduaçào mediclas commus para serem comparadas às competências exigidas (htlp://www.kaplan.com1). Utilizando a metodologia de Teste Adaptativo. foi criado o GRE CAT alja avaliacaio considera o raciocínio vexbal. (quantitativo e analítico do estu-

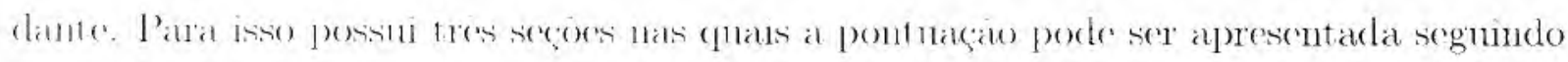

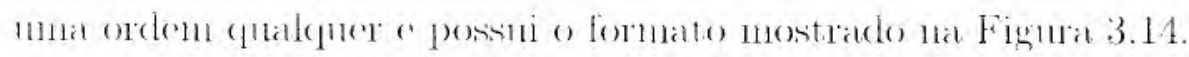

\begin{tabular}{|l|c|c|}
\hline \multicolumn{1}{|c|}{ Seção } & Tempo & $\begin{array}{c}\text { Quantidede de } \\
\text { Questoes }\end{array}$ \\
\hline Verbal & 30 minutos & 30 \\
\hline Guantiativa & 45 minutos & 28 \\
\hline Logica & Bominutos & 35 \\
\hline
\end{tabular}

\section{Fignara 3.14: Secoion estrutura do (iRE CAT}

As áreas testarlas por estaśs secoès seguem o seguninte conteńdo:

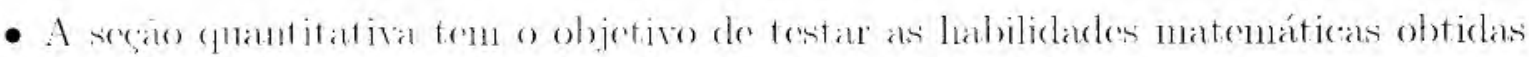

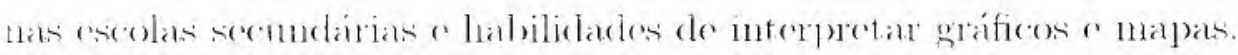

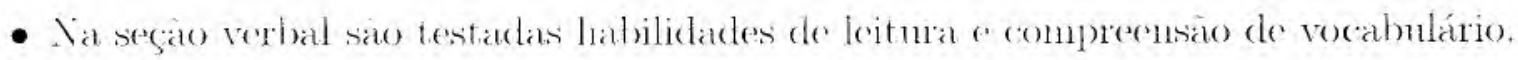

- A seção analítica testa o raciocínio lógico ntilizando jogos.

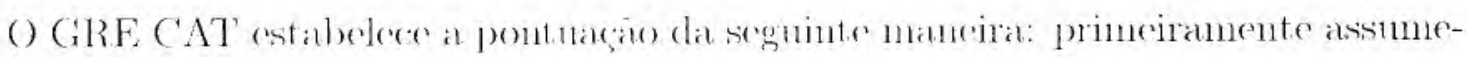

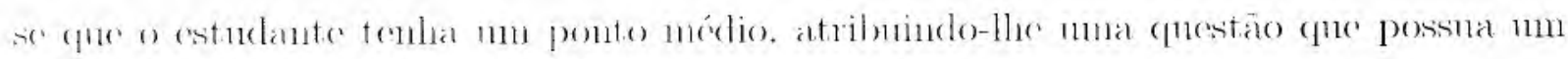
gran de dificuldade considerado médio. Daí por diante ó procediclo como mostrado na Figura 3.15. 


\begin{tabular}{|c|c|}
\hline $\begin{array}{l}\text { Se o estudante } \\
\text { responde el questabo } \\
\text { corretamento }\end{array}$ & $\begin{array}{l}\text { Se o estudante responde } \\
\text { a questao incorretamente }\end{array}$ \\
\hline 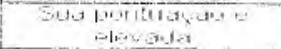 & 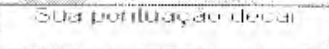 \\
\hline 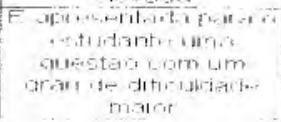 & 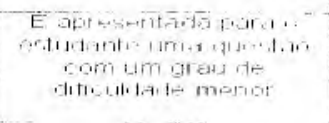 \\
\hline
\end{tabular}

Figma 3.15: Estabelerenclo a pontuacio no GRE CAT

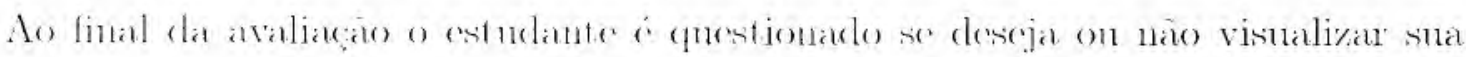

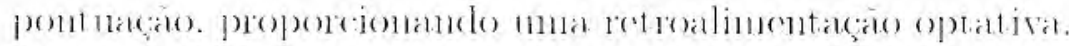




\section{Capítulo 4}

\section{Taxonomia de Objetivos Educacionais de Bloom}

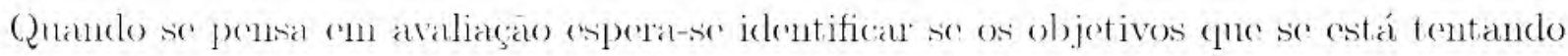

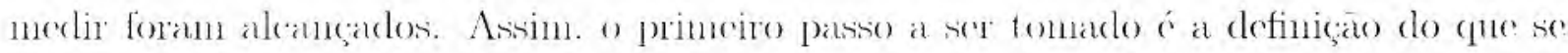

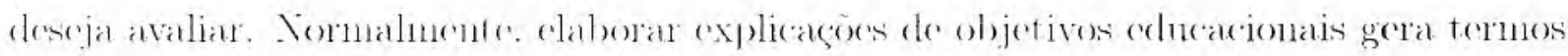

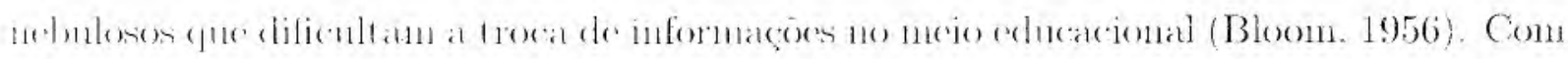

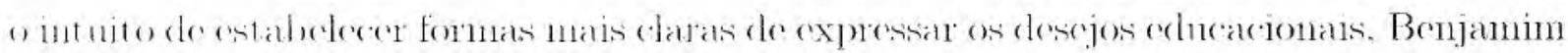

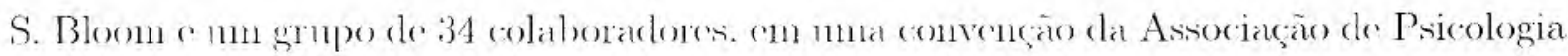

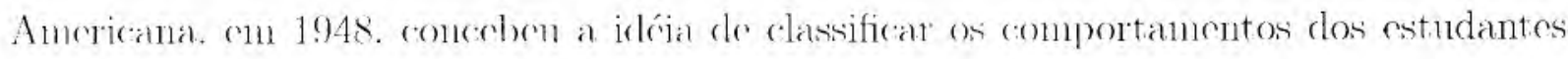
fun representam os objetivos do processo edncarional. Tres domínios de atividades foran identificados: Dominio Cognitivo. Domínio Afrtivo e Domínio Psiconotor. Como a Taxonomia do Domínio Cognitivo teve a continna tenelo grande influcneia no projeto de

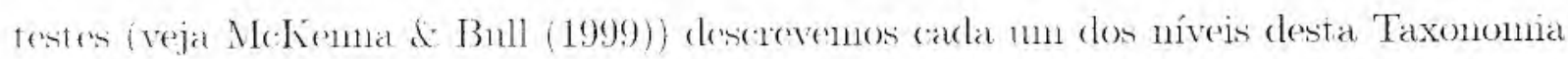
gue é conlereda cono Taxononia de Bloom. Esta Taxomonia tenta dividir os objetivos

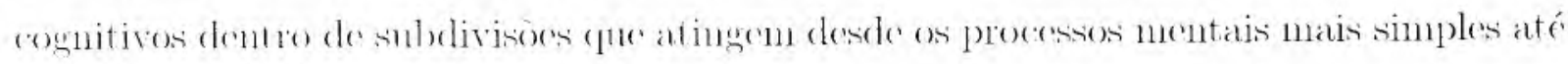
us matis complexus.

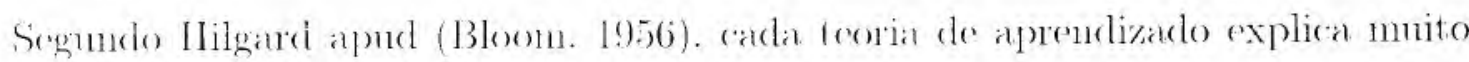

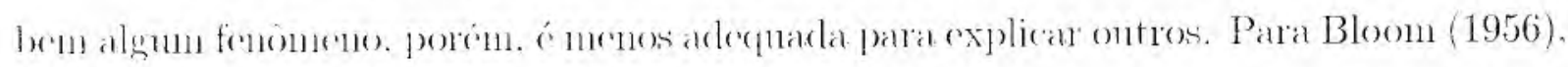

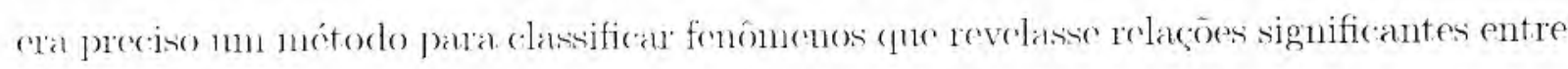
eles. Este ó o problema básico tratado perla Taxonomia - classificar um fenomeno de ma- 


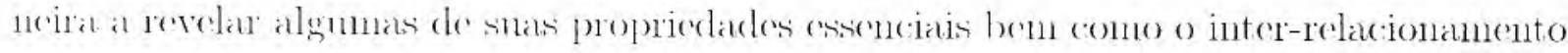

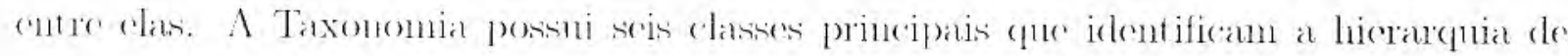

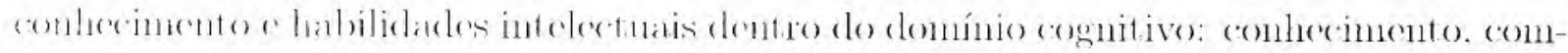

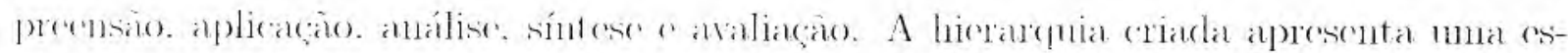
trutura útil a simples para ser utilizada na identificagáo dos resultados do aprendizado. Carla classe será descrita brevemente. focalizando os pontos relevantes no que se refere ao comportamento do estuclante enu termos do processon mental esperado. Serão apresentadas

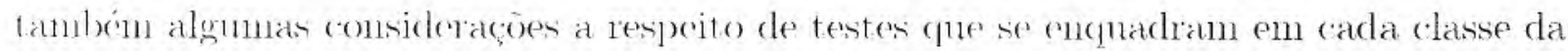
litxonomia o os verbos sugeridos para serem inplicita (induzen ao conportamento) on

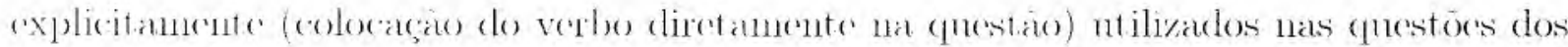
1.esties.

\section{Classe Comberimento}

\section{Refere-se a}

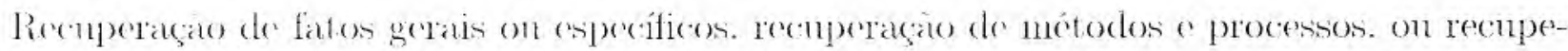

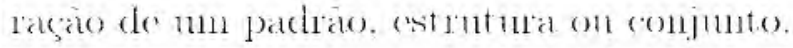

\section{Processo Mental}

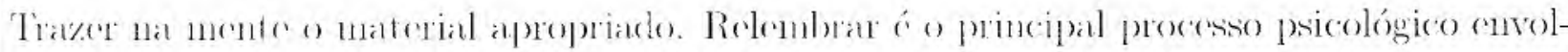

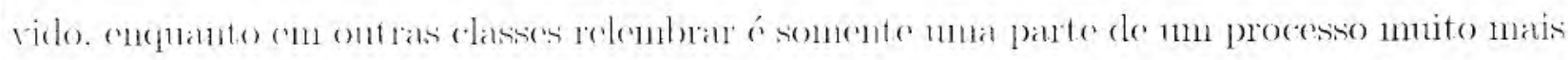
(o)

Divisão da Classe Couleczumento

\section{1 - Conhecimento de informações específicas}

\section{Refere-se a}

Tipos de informacào on conlecinento ene pode estar isolado e ser relembrado separadamente.

\section{Subdivisōes}

1.1 - Conlecinento de terminologia

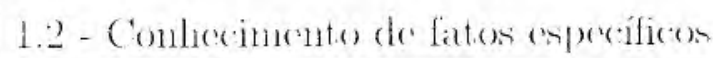




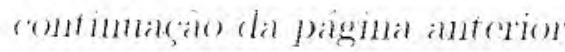

Classe (onhermento

2 - Conhecimento de formas e meios de se tratar informações específicas

Refere-se a

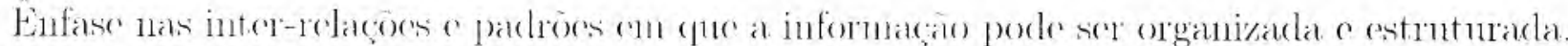

Subdivisões

2.1 - Conherinuento de convencouses

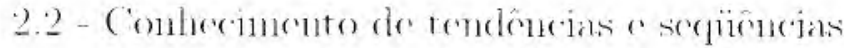

2.3 - Conhecimento de classificacoes c classes

2.4 - Conhecimento de (ritérios

2.5- Conleerinerite de metodologia

3 - Conhecimento das abstrações em um campo do conhecimento

Refere-se a

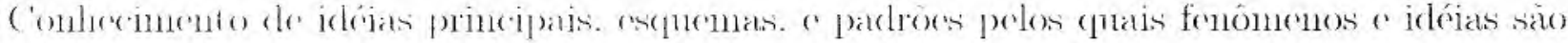
organizarlos.

\section{Subdivisões}

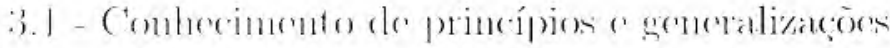

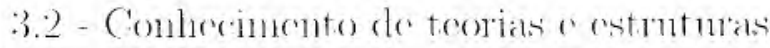

Tabela 4.1: Taxomonia do Bloom - Combermentos

\section{Categoria (onhrorimento - Consideraçoess a respeito de Testes}

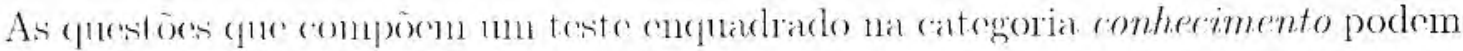

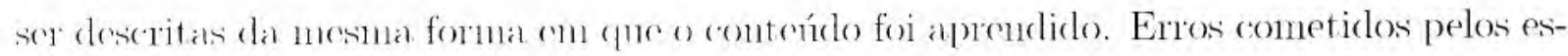
fudantes podem sere niteis para o planejanento de alternativas de escollha para as questões. In resumo do tais erros podo ser ítil para precisar o conlecimento do estridante.

Verbos ilustrativos utilizados nas questões dos testes da categoria do combermento: definir, descrever. enuncrar, identificar. nomear. listar, associar, recordar, reproduzir. selecionar. reconhecer. relatar. resperar, repetir, informal. 


\section{Classe Compufension}

\section{Refere-se a}

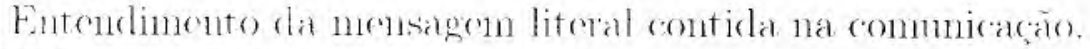

\section{Processo Mental}

()is estudantes saio confiontados com alguma forma de commicaçào (oral on escritad. verbal on

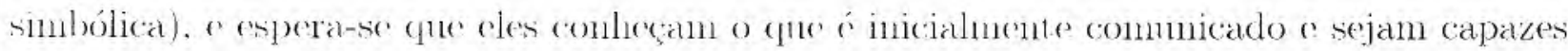

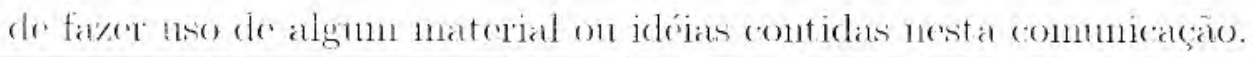

\section{Divisão da Classe Comprernstio quanto aos tipos de processos mentais}

\section{1 - Tradução}

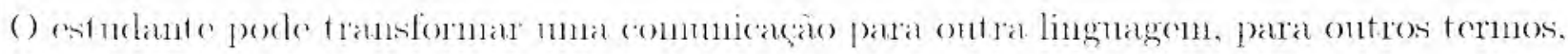

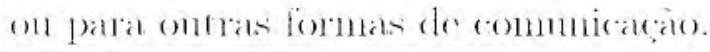

\section{2 - Interpretação}

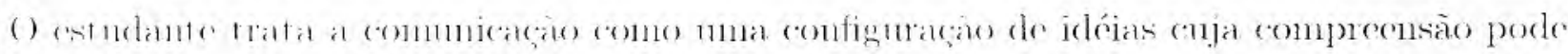

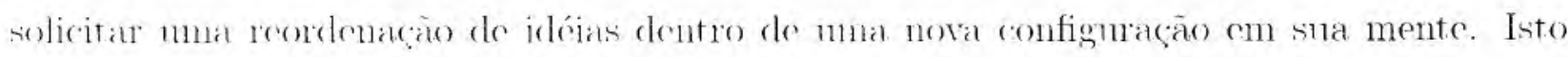
inclui também pensamentos sobre a importância relativa das idéias. sllas inter-relaçoes, e sna relevancia para generalizaçes implicitas on descritas na comnnicação original. Evidências

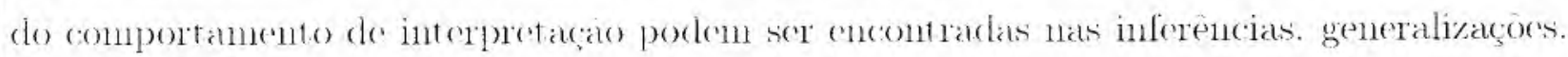

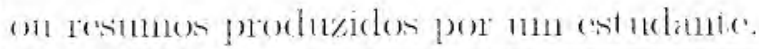

\section{3 - Extrapolação}

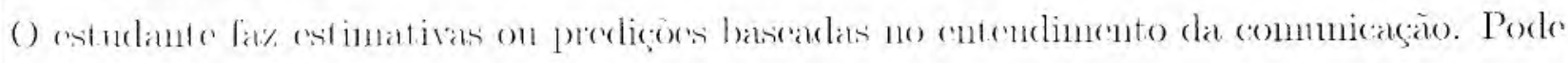

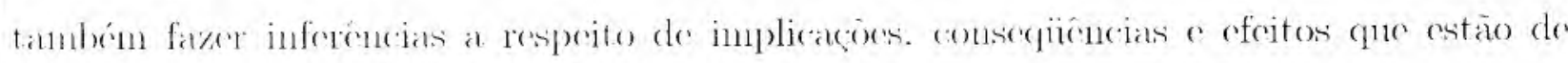

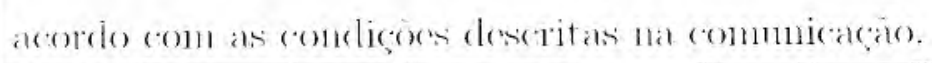

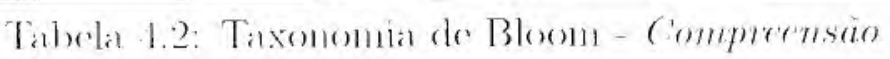

\section{Categoria Comprernsaio: Tradução - Considerações a respeito de Testes}

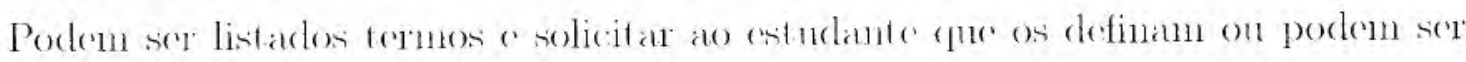

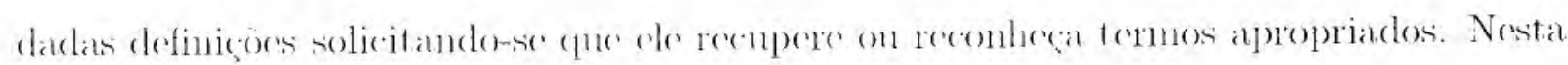

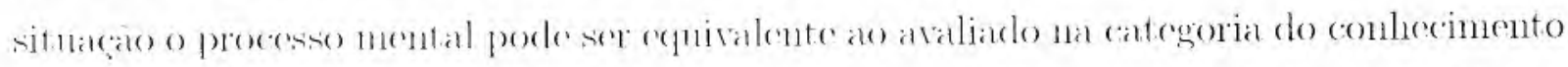

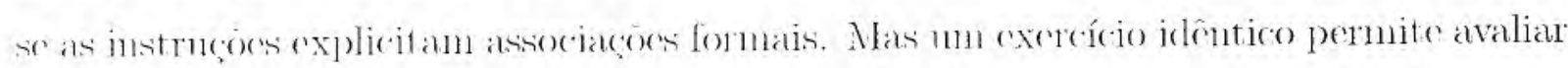




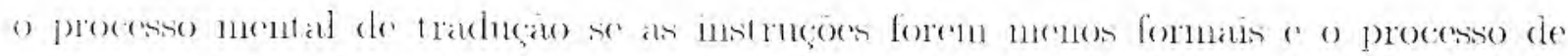

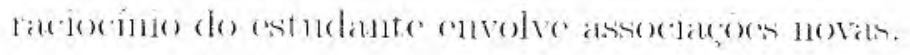

Categoria Comprernsaio: Interpretação - Considerações a respeito de Testes

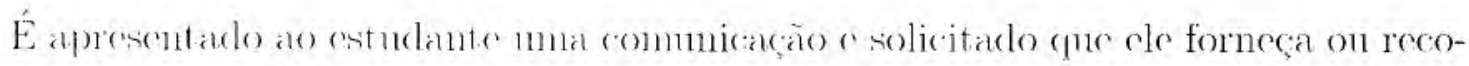

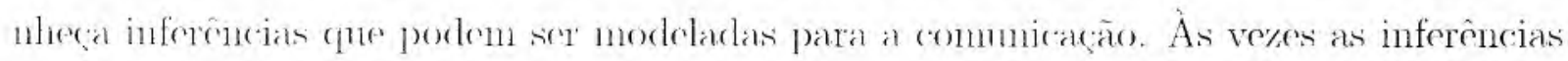
pedem repuesentar generalizacoes on poden perteneer a particularidades em uma dada

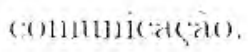

Categoria Comprentusco: Extrapolação - Considerações a respeito de Testes

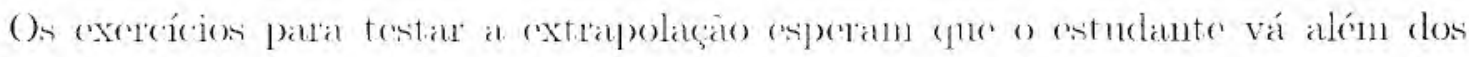

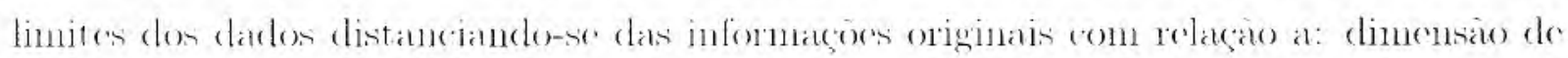

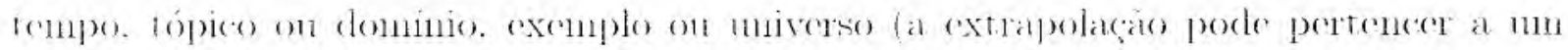

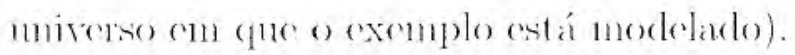

Verbos ilustrativos utilizados nas questões dos testes da categoria Com-

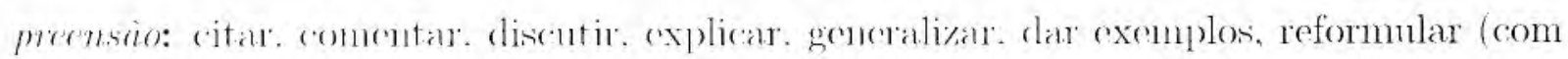
slats próprias palarras). traçar, revisar.

Classe 1pluaturen

\section{Refere-se a}

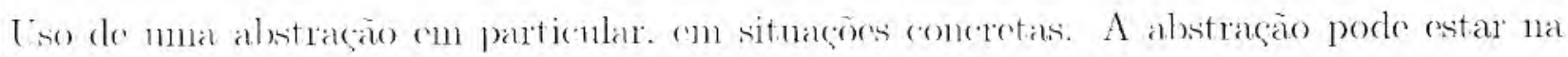

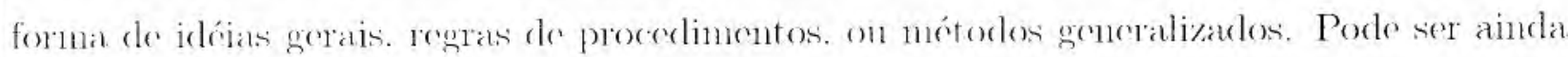

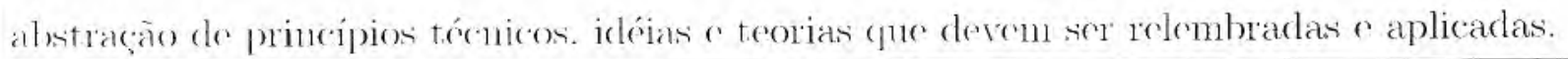




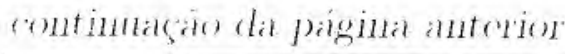

Classe Apherescos

\section{Processo Mental}

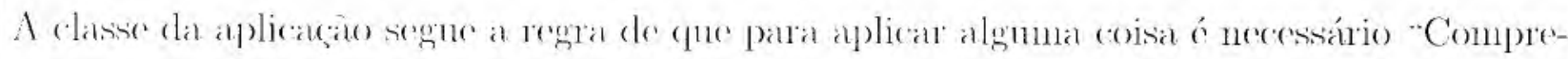

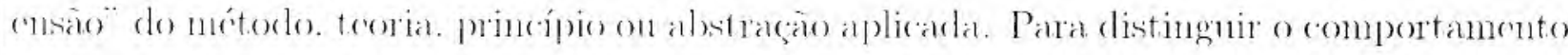

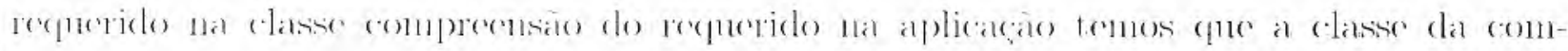

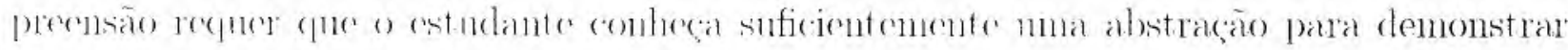

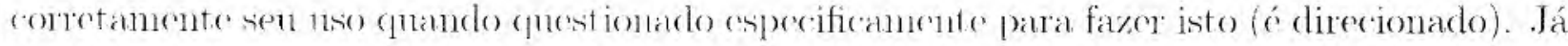
a aplicação requer (que clado nun novo problema, o estudbunte aplique a abstração apropriada sem ser direcionado on sem ter siden mostrado conno ntsá-la on em que sitnaçăo niśá-la.

Tabela 4.3: Taxonomia de Bloon - Aplicascao

\section{Categoria Aplicaça - Considerações a respeito de Testes}

Tostes para categoria Aplecacao possitem algums fatores rotineiros:

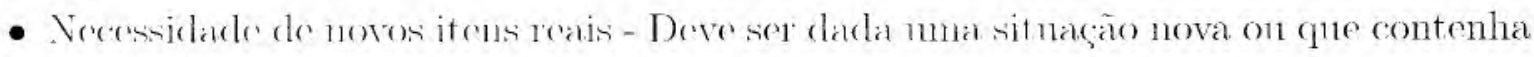

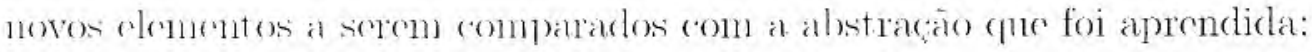

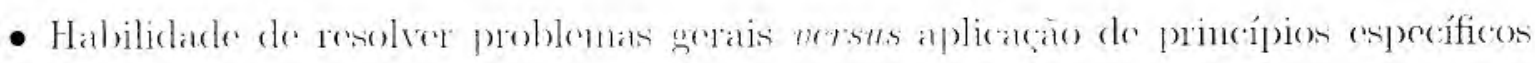

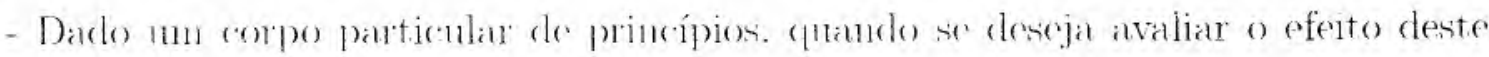

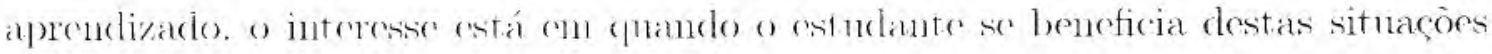
específicas. Problemas que contém pistas de como soluciona-los não testariam apliarcoes de princípios que formun ensinados:

- Diagnosticar falhas em problomas de aplicacão - Algmuas cantas podem explicar o porene do estudante náo ter resolvido problemas de aplicacão: a) compreensão

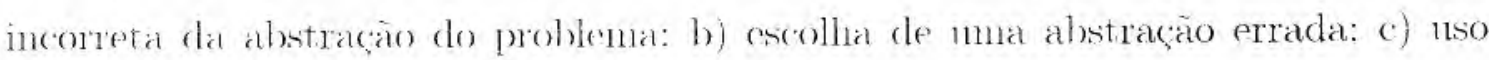

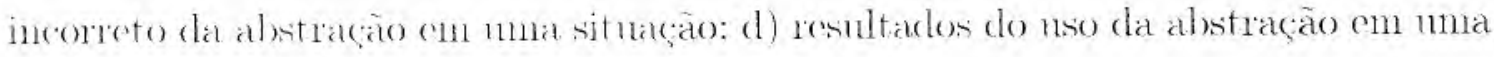
situacio interererados incorretanuente.

Verbos ilustrativos utilizados nas questões dos testes da categoria apli-

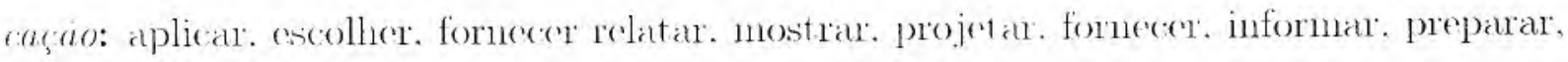
estabelecer. planejas. 


\section{Classe Ancílise}

\section{Refere-se a}

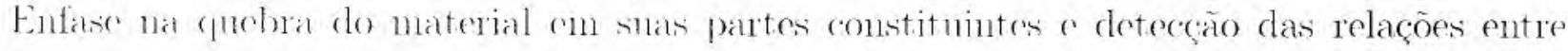
at partes e da maneira que rstäo organizadas. A análise trata do contendo e da forma do material

\section{Processo Mental}

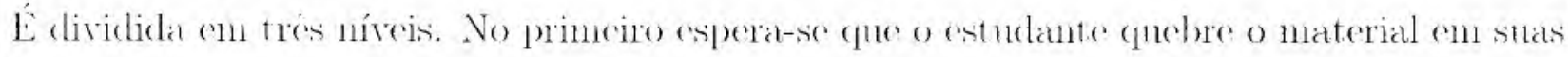

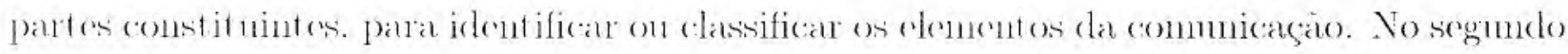

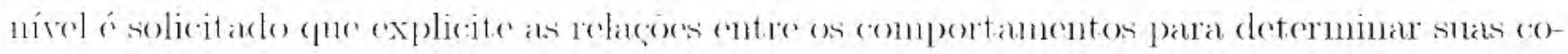

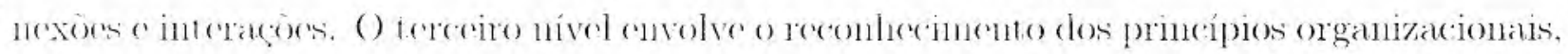

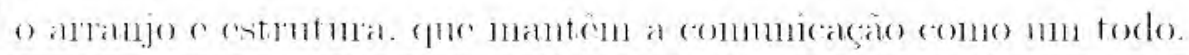

\section{Divisão da Classe Anúlise}

\section{1 - Análise de Elementos}

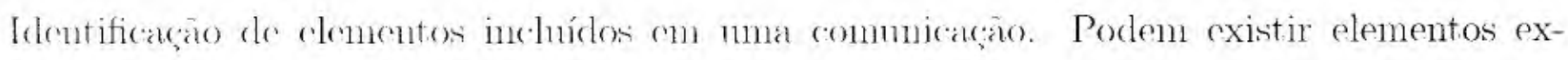

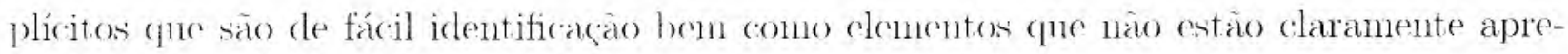

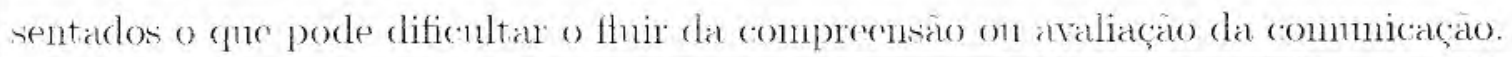

\section{2 - Análise de relacionamentos}

Conexoes e interaçoes entre elementos e partes da communicaço.

\section{3 - Análise de princípios organizacionais}

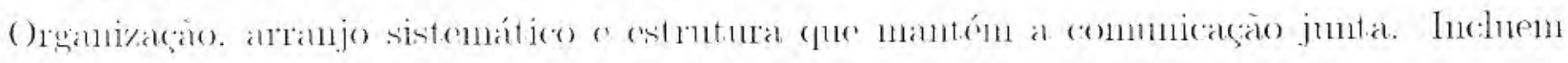

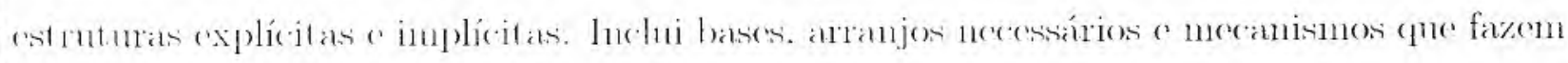
da comminicatoto mon midarle.

\section{Tabela 1.4: Taxomomia de Bloom - Análise}

\section{Categoria Anúlisf - Considerações a respeito de Testes}

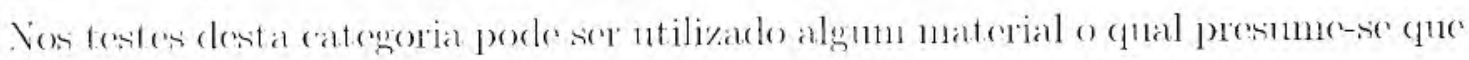

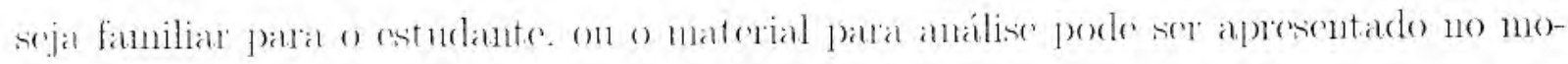

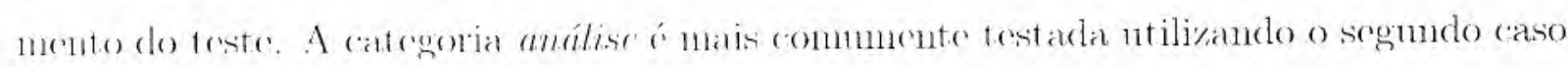

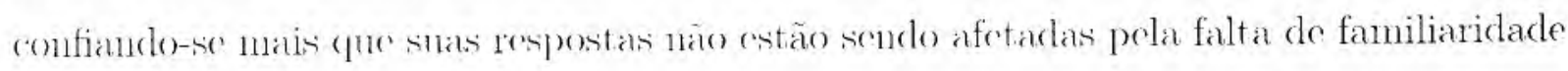




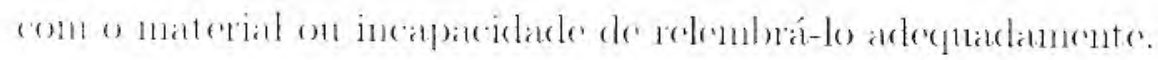

Verbos ilustrativos utilizados nas questões dos testes da categoria análise:

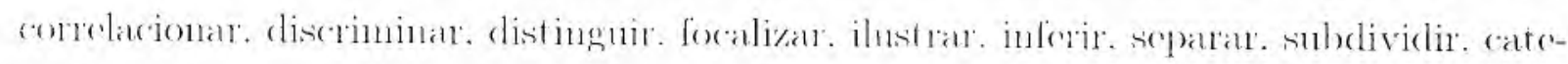
gorizar. contrastarr. distmentir.

Classe Sintese

Refere-se a

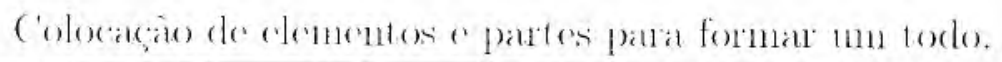

\section{Processo Mental}

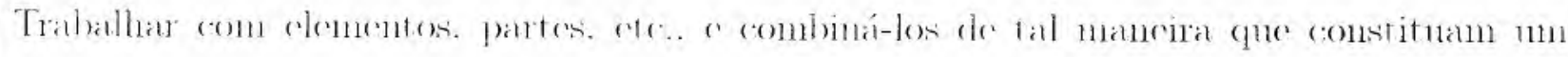

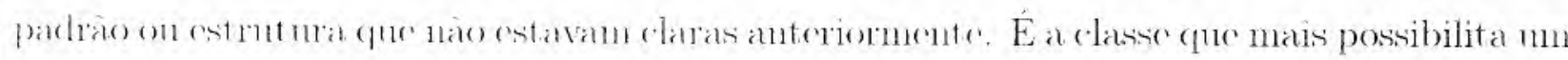

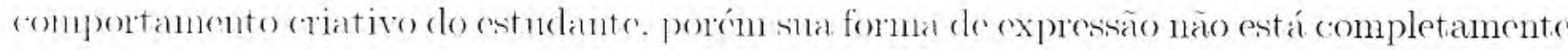
livere pois gerahnente espera-se que trabalhe dentro de conjuntos limitados por um problema emp particular. materiais. on algunna estruttua teórica on metodológica.

Divisão da Classe Sintese

1 - Produção de uma única comunicação.

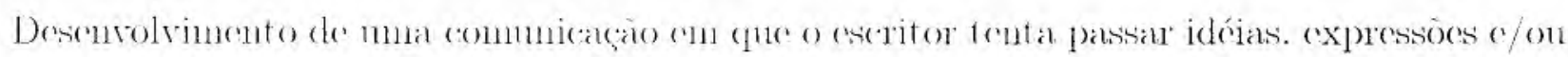
(xperiencials parar ontros.

2 - Produção de um plano, ou conjunto de operações propostas.

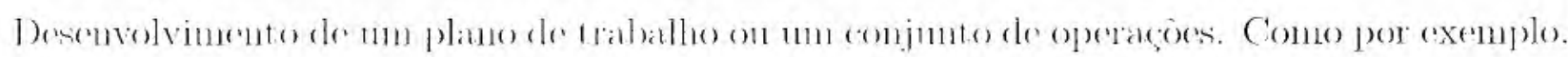

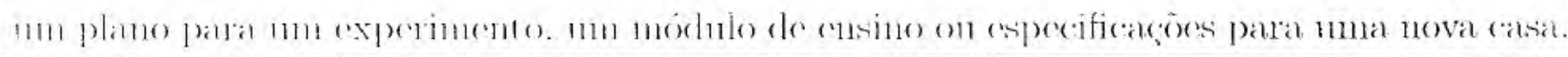

3 - Derivação de um conjunto de relações abstratas.

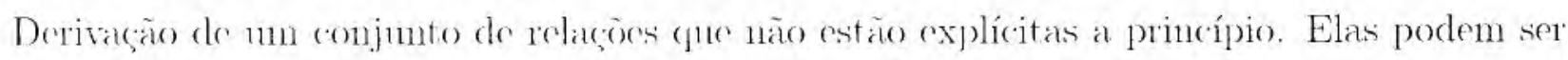
derivarlas de amálise de fenômenos on hipóteseses a serem testados.

Tabola 4.5: Taxomomia do Bloom - Sintese

Categoria Simtest - Considerações a respeito de Testes

O principal problema de testes da categoriat síntese ć proporcionar condiçoes fávoráveis para num trabalho criativo. Talve\% a principal condição seja a liberdade. Tal liberdade poele estar delinitada pela inclusão de 1 m ponto de vista adotado em parti- 


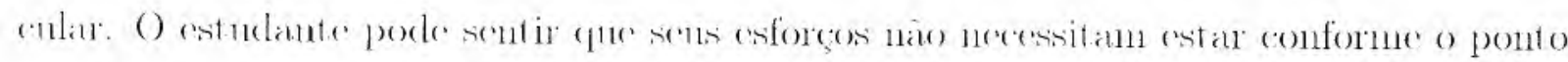

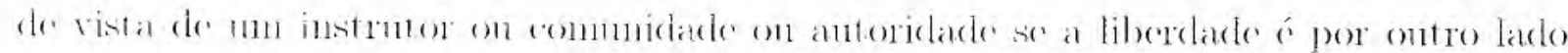

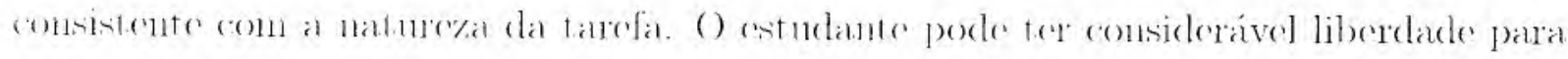

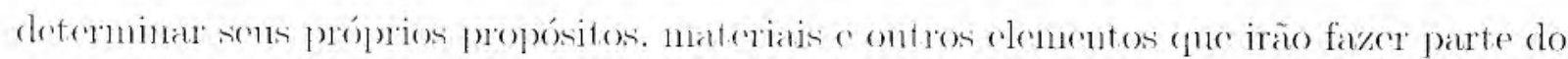
(fure cstá produrindo.

Verbos ilustrativos utilizados nas questões dos testes da categoria sintese: (ombinar. compon. (ontrastar. criar. modelar, expresisar. dritenvolver, formular. organizar.

Classe dialuercion

\section{Refere-se a}

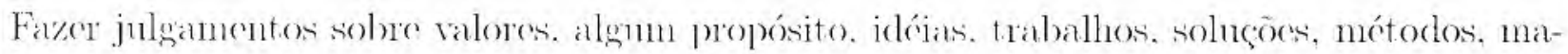
terial. etc: ()s julgannentos estäo bascados ene 1 mu critério definiclo.

\section{Processo Mental}

Envolve numa combinacao de todos os ontros comportanentos de conhecimento compreensáno.

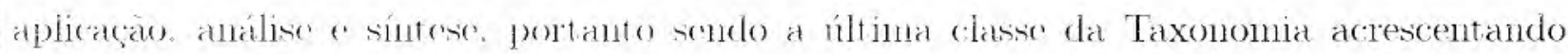
critcrios gure inchuene valorest.

Divisão da Classe Avaliaçao

\section{1 - Julganento em termos de evidências internas.}

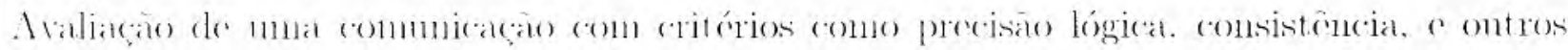
(ritérios internos.

\section{2 - Julgamento em termos de critério externo.}

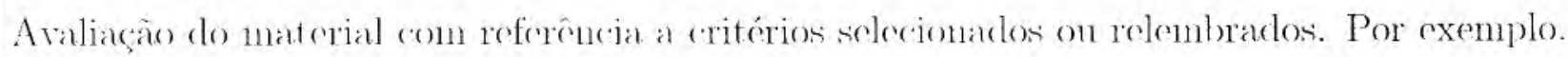
a habiliclade de comparas um trabalho com traballos de reconhecida excelência.

Tabela 1.6: Taxomomia de Bloom - Avaliaçaco

\section{Categoria Avalimsa - Considerações a respeito de Testes}

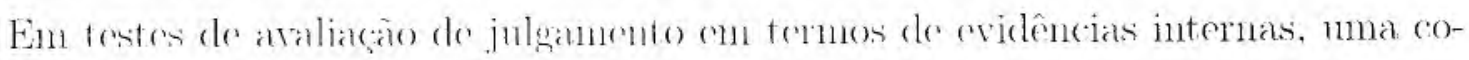

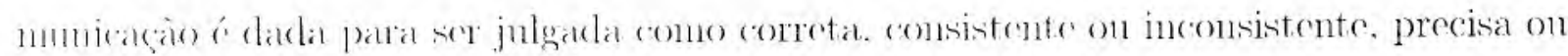

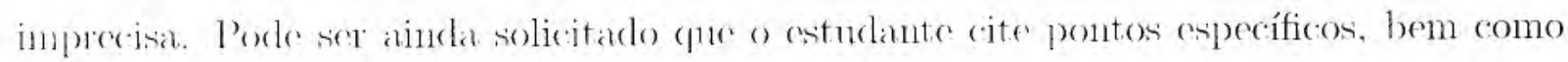
as lazós pelas quais ole julgon de tal maneira. Nos testes da categoria avaliação com 


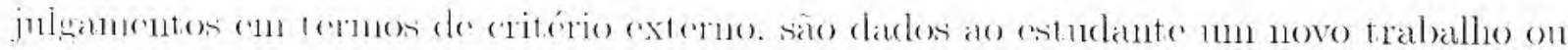

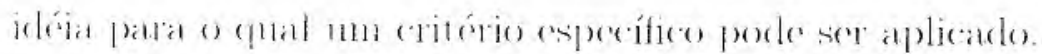

Verbos ilustrativos utilizados nas questóes dos testes da categoria ava-

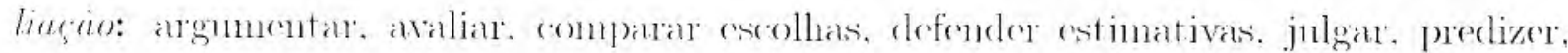
selecionar. conednit, criticar. defeneler, justificas.

Resmmiclamente as classes poreme ser vistas da segunte forma:

\section{Conhecimento (Memorização)}

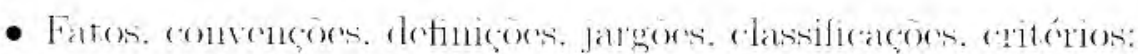

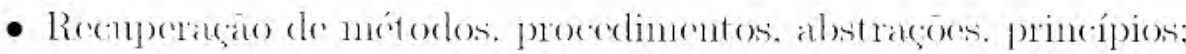

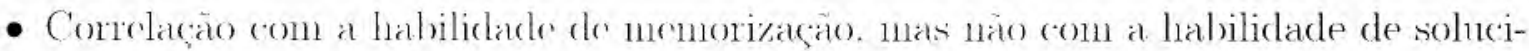
onar poblentais.

\section{Compreensão (Entendimento)}

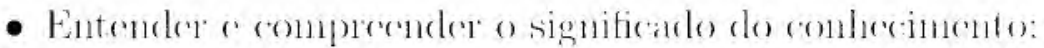

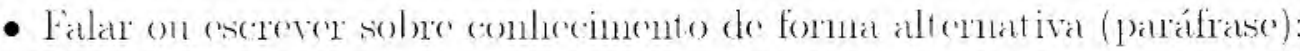

- Articular conexoes contre itens rliferentes do conheciemuto:

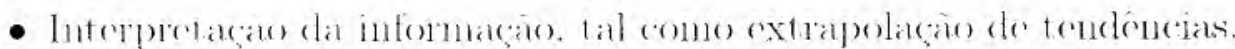

\section{Aplicação (Uso)}

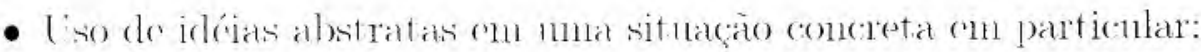

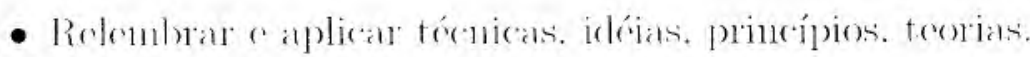

\section{Análise (Dividir em partes)}

- Dividir 1 un problema complexo cou partes:

- Solncionar cada parte ntilizando princípios, teorias. ete:

- Determinar conexoes o interacoses chetre pautes.

Síntese (Reunir as partes) 


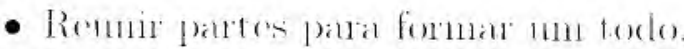

\section{Avaliação (Julgamento)}

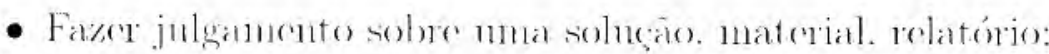

- Poder anvolver aritário intermo on externo:

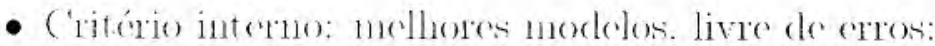

- Critérios externe: ambiental. legal. seonomicos sociológicos.

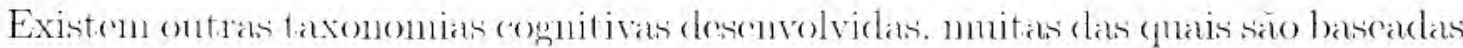

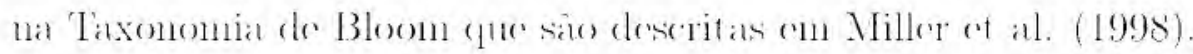

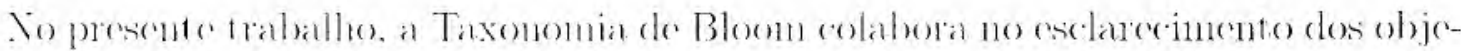

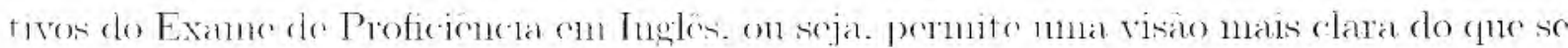

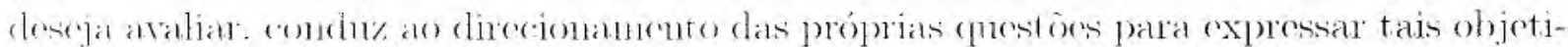
vos e pessibilita que a avaliaçào seja mais halancrada en termos da composiçào de vários objetivos enu voz de sobrecarregá-la. por exemplo. (om ob jet ivos que requeiram processos nurutais mais emplexos on mais simples. Por meio de exemplos de questón's a respeito de

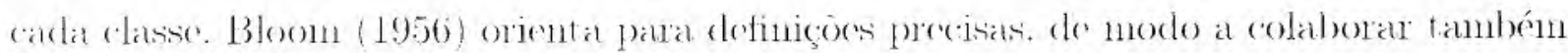

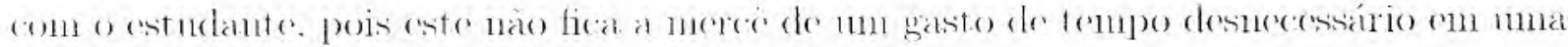

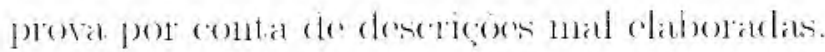




\section{Capítulo 5}

\section{Testes baseados na Medida de}

\section{Probabilidade Admissível (MPA)}

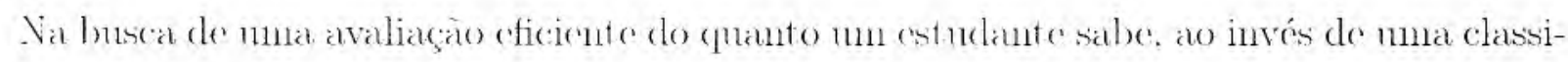

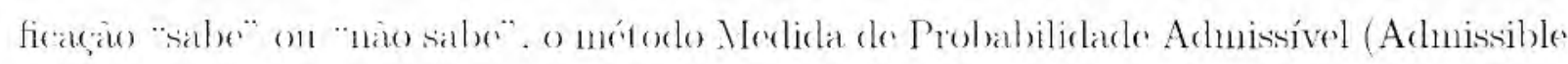

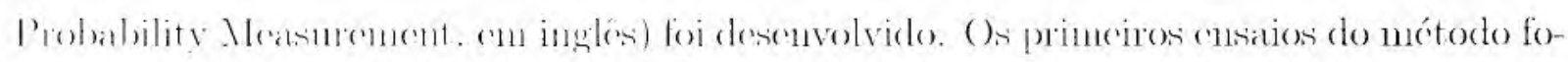

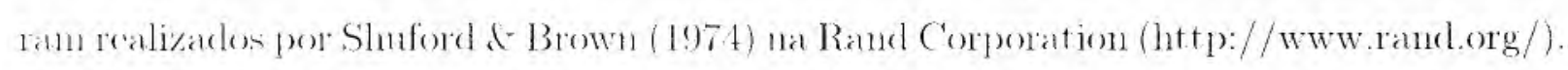

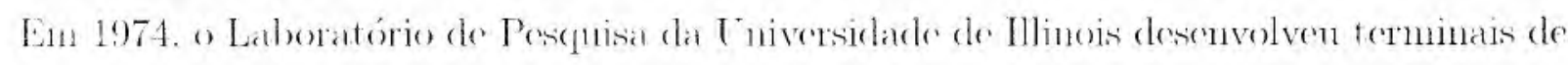

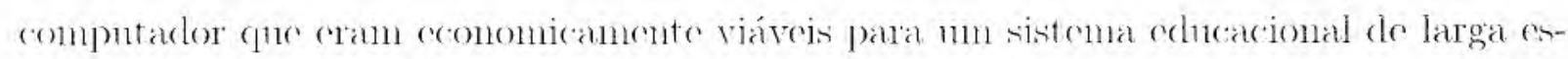
cala (Sibley 1974). Com estes terminais, desejavam desenvolver mula versão da MPA incluindo mua coleção de dados apropriados, análisess e implementação de pacotes para servicos militares. Desenvolverann. entäo. $14 m$ protótipo en que o teste poderia ser editado nos moldes de nétodo Medicla do Probabilidade Adnuissível (MPA) e o estudante poderia fazer o teste respondendo is chestoes viat terminal.

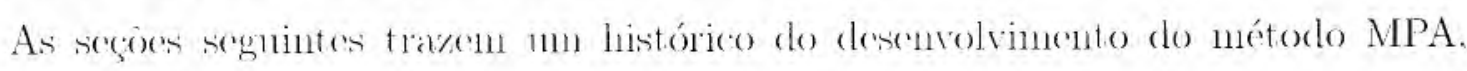
relatando alguntis experioncias ocorriclats com o mesmo.

\subsection{Criação do método MPA}

Shunforl \& Brown (1974) so interessaranu pela pescuuisa de novas motodologias para medir com maior precisão o estado do conbecinento (o q11anto o cutudante sabe) de um estudante 


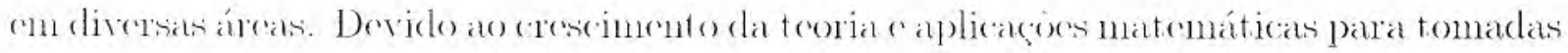

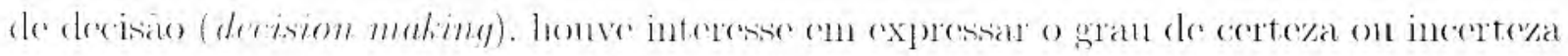

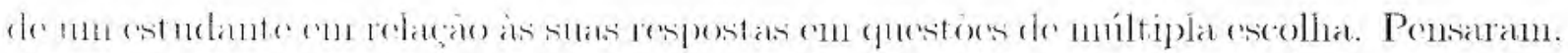

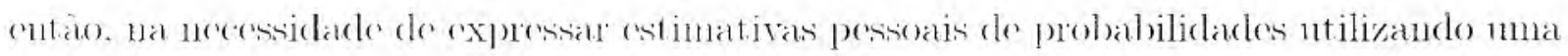

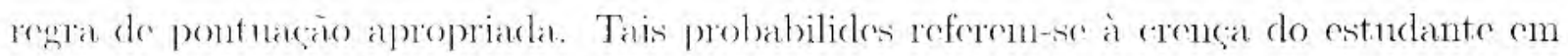

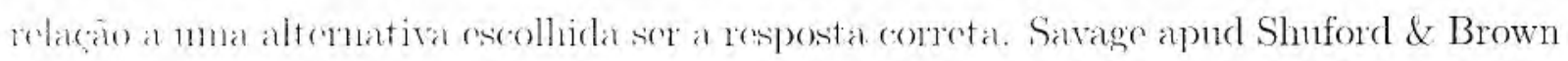
(1974) referencia o poteneial educarional existente con teste de múltipla escollat. em que o

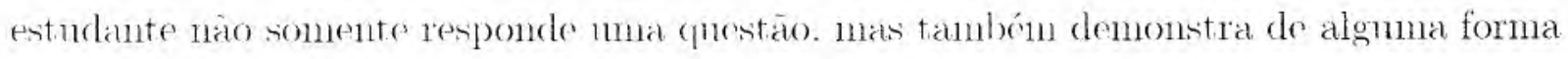
(onno sua opmina está distribuida solne as opçoes de respostas. Se o estudante responde

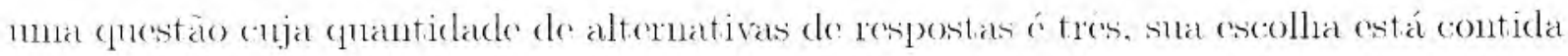

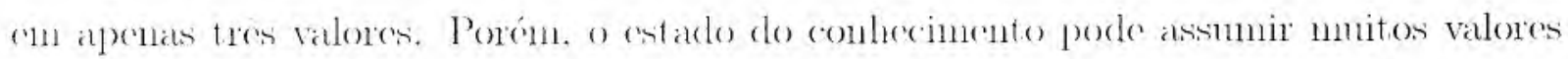

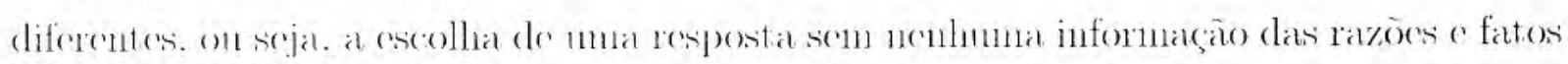

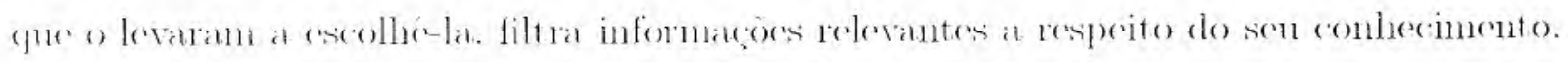

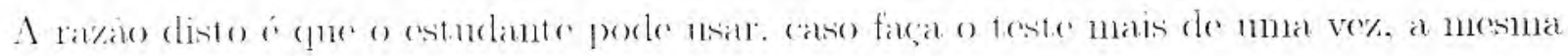
Jespostat para estades de conherinemendiforentes. Para Shufored \& Brown (1974) era

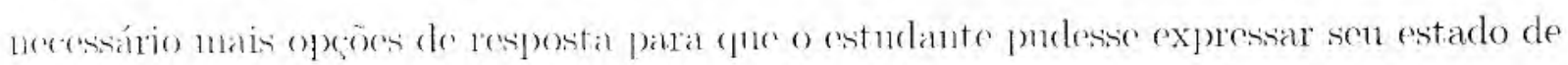

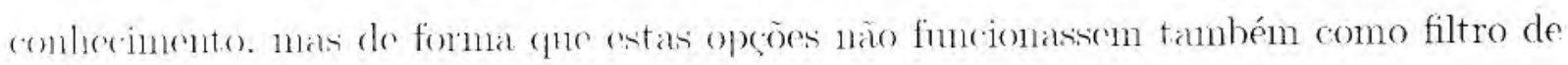
informacồ importantes. A alternativa foi permitir (1ure o estudante associasse um peso à resposta escolhida expressando a probabiliclade de sud escolha ser a resposta correta. Em

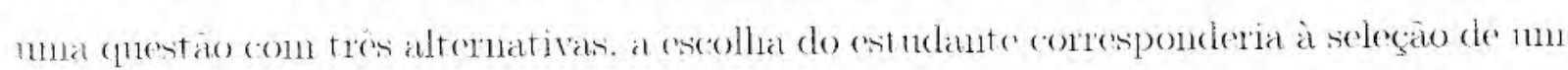

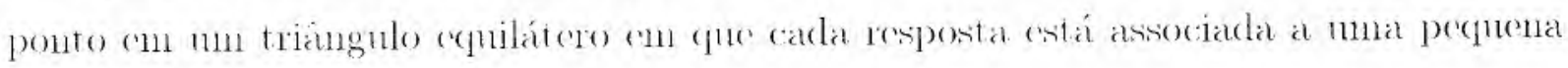
negiào de probabilidakles.

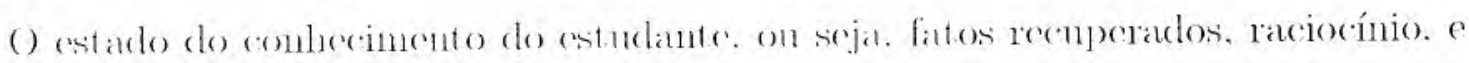

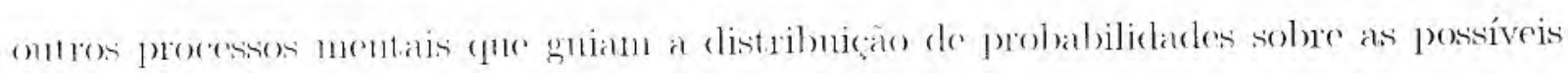

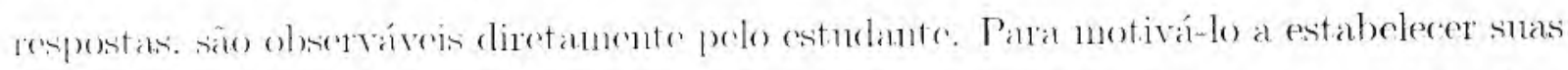

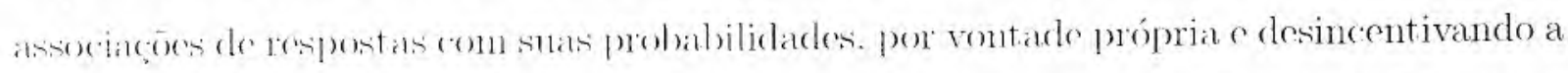

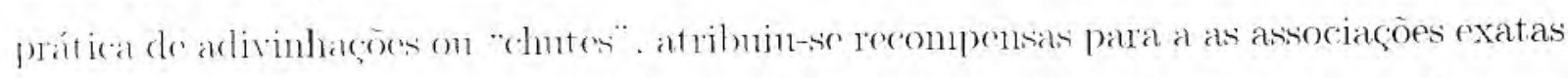
e consistentes rom a resposta correta e penalidades para as associaçoes inconsistentes. Desta forma. o estudante tem o descejo de maximizar a silla pontulação, utilizando para isto 1111 processo de tomada de decisio que. para cada questaio. selecioma a resposta que ele 


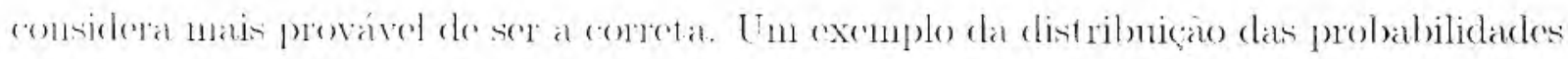

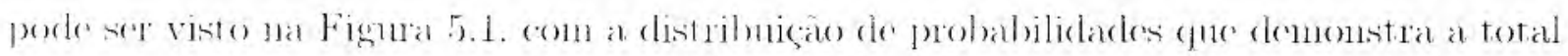
distincăo de muna altermativa das dematis.

\begin{tabular}{|l} 
O décimo primeiro presidente dos Estados Unidos foi: \\
$\begin{array}{cll}0 & \text { 1. George Washington } \\
1 & \text { 2. James Polk } \\
0 & \text { 3. Franklin Pierce }\end{array}$ \\
$\begin{array}{ll}\text { Distribuição } & \\
\text { de Probabilidade }\end{array}$
\end{tabular}

Figura 5.1: Distribuicâo de probabilidade demonstrando total distinçâo entre uma alternativa

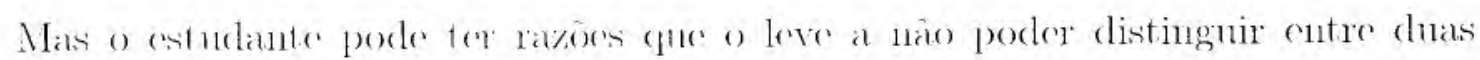

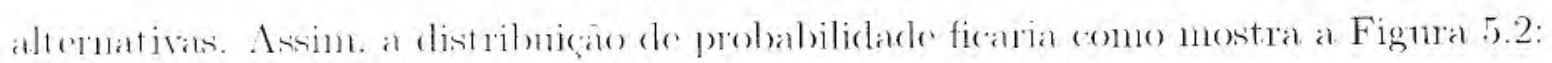

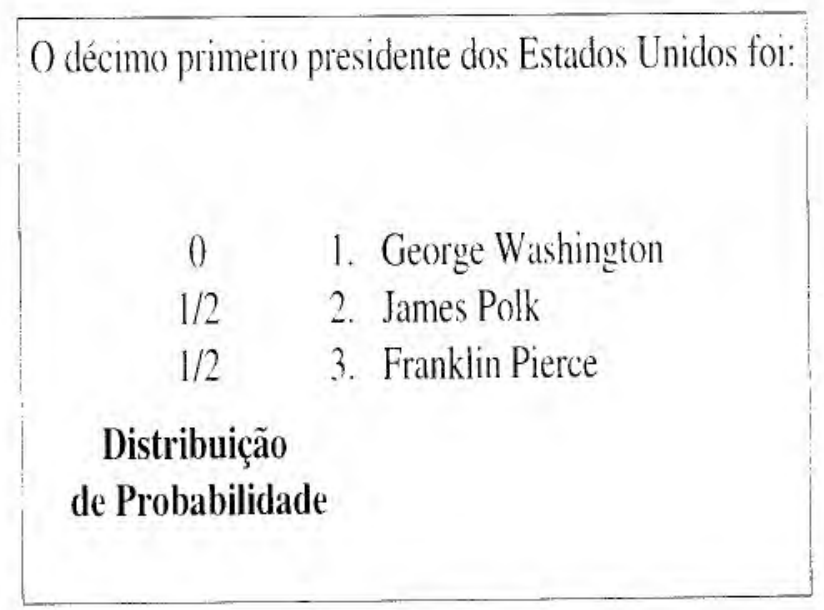

Figura 5.2: Distribuição de probabilidade (1uando năo se distingue entre duas alternativas

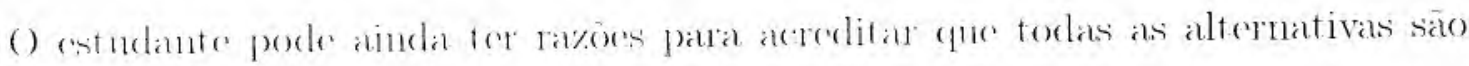

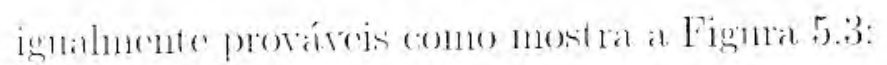




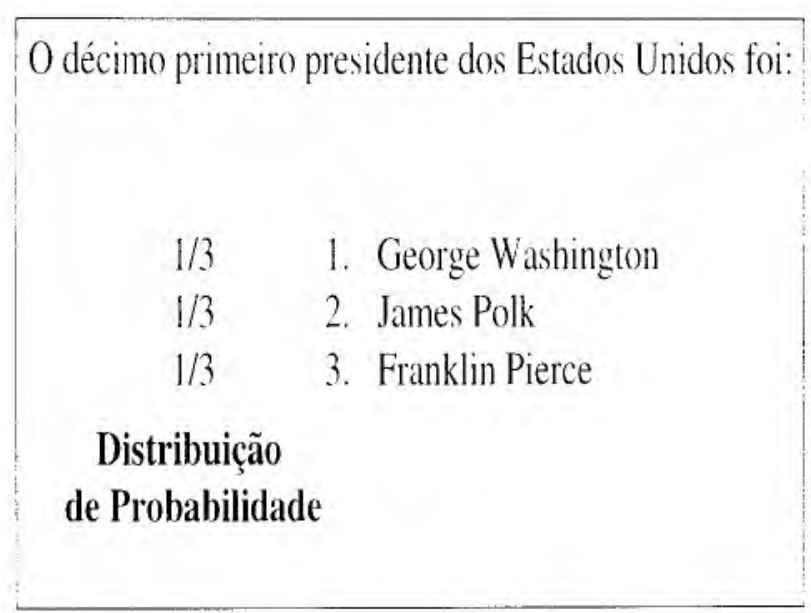

Fignra 5.3: Distribuicâo de probabilidade quando nâo se distingne entre três alternativas

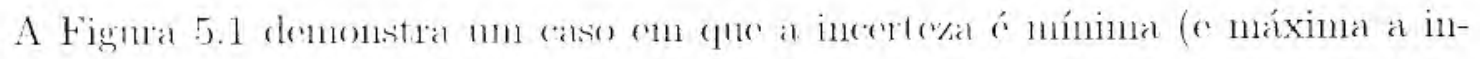

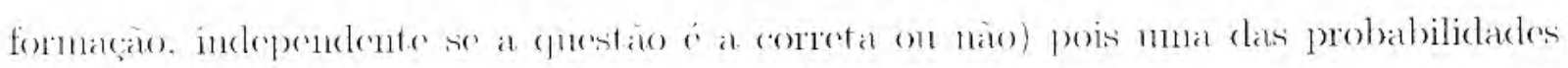

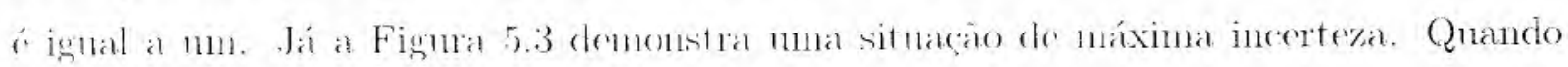

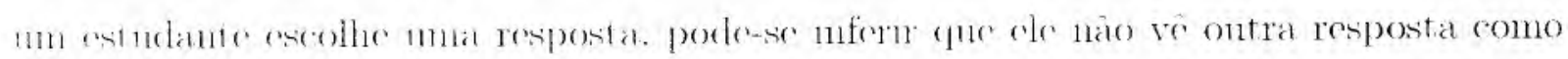
mais provável do ser a correta. Termos como "Bem informado". "Desinformado" e "Não informado" săo as vezes utilizados para descrever o conlıecimento pessoal sobre algum assinto. Shuford s Brown (1974) ntilizan tais termos para caracterizar as regióes dos apaces de probabilidades 110 triangulo equilátero de tres possiveis respostas como mostra a Figura 5.4.

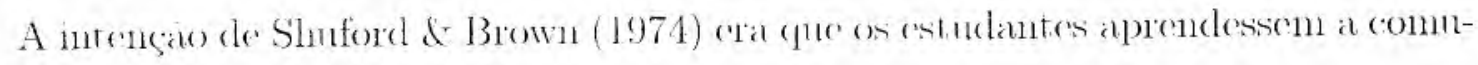

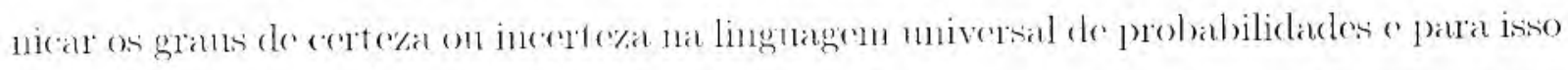

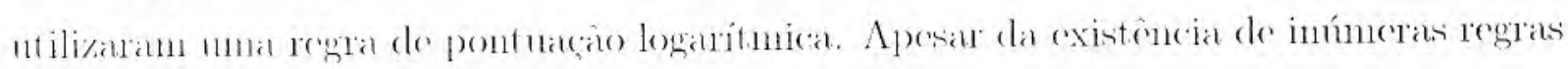

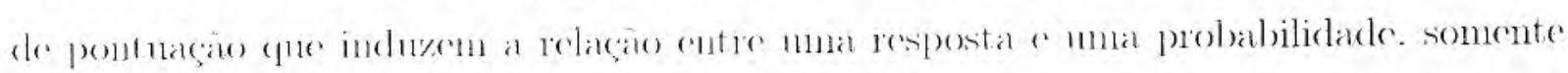

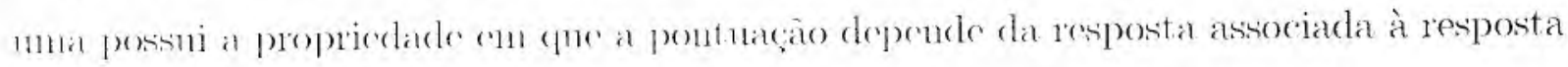

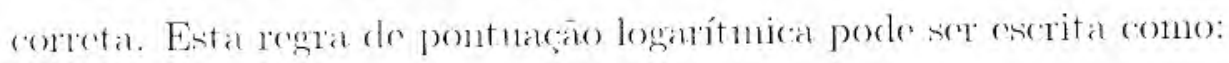

$$
S_{i}=A \times \log r_{i}+B
$$

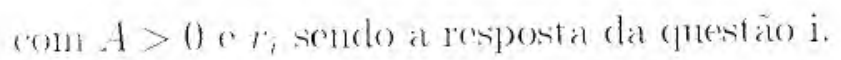

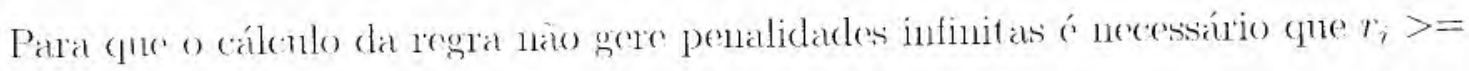

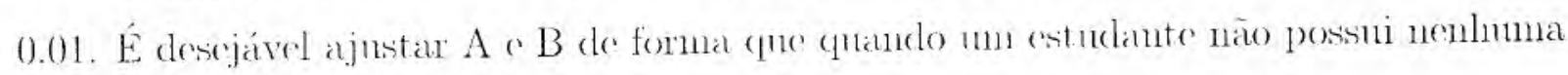




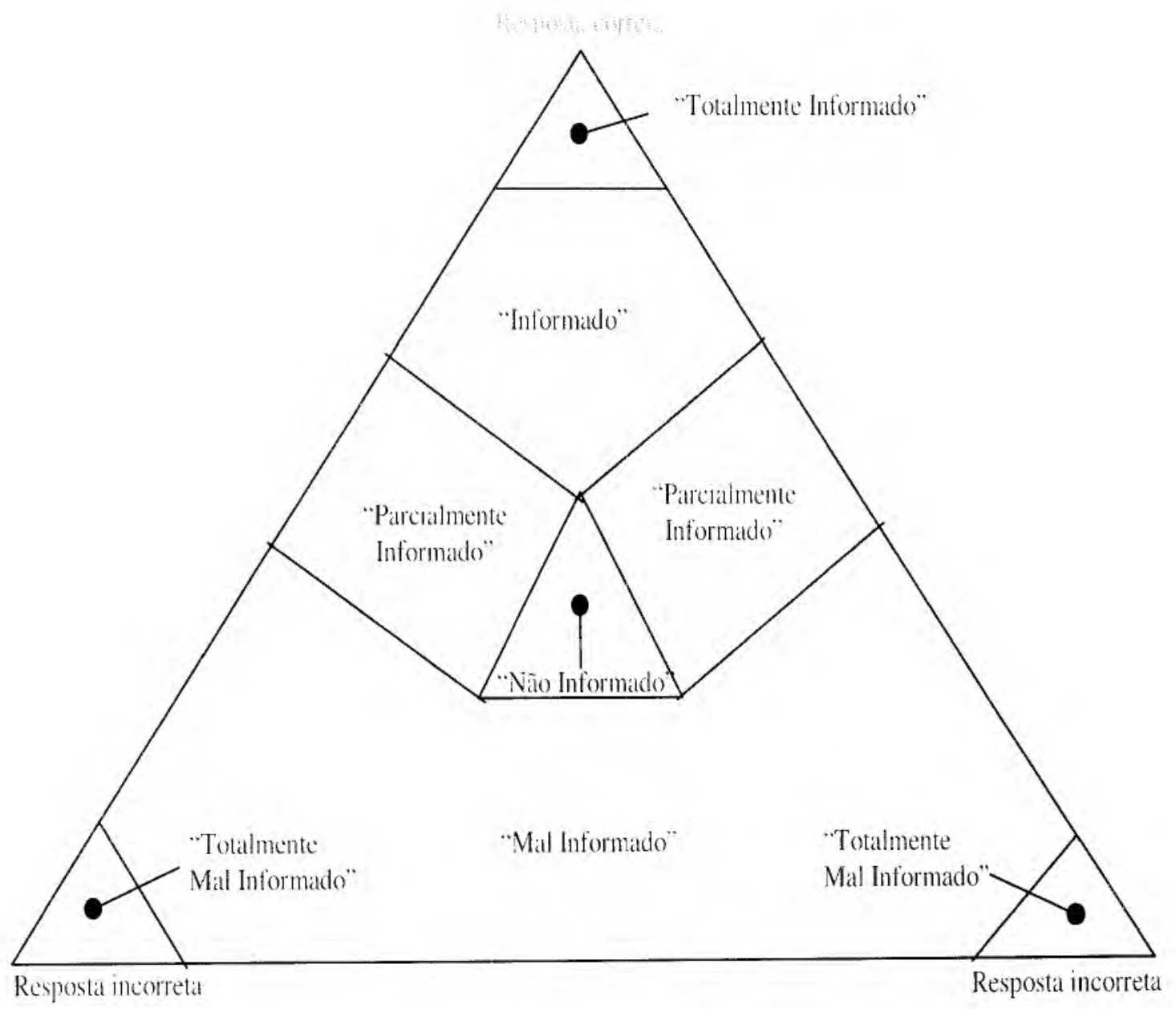

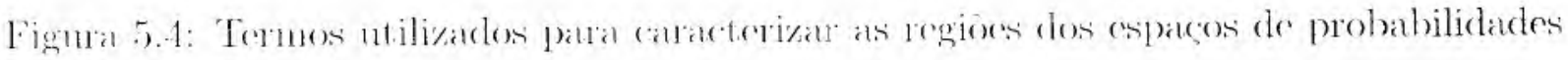
Shunford \& Brown 1974).

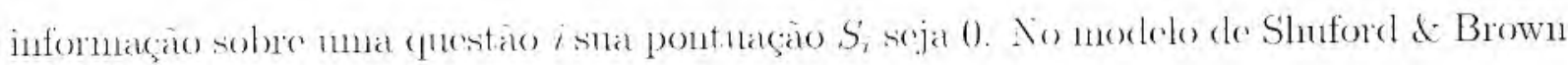

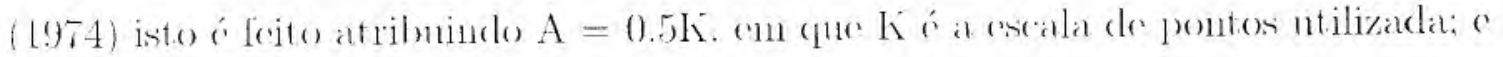

$$
B=0.5 \text { Klogk ri }
$$

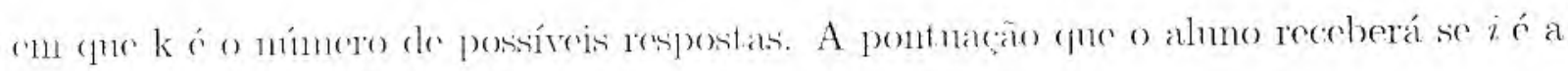

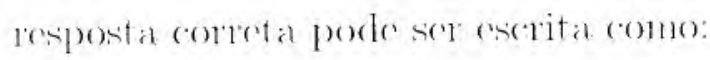

$$
S\left(r_{i}\right)=0.5 \mathrm{~K} \log \text { lir }
$$

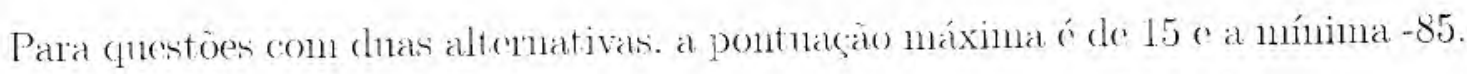

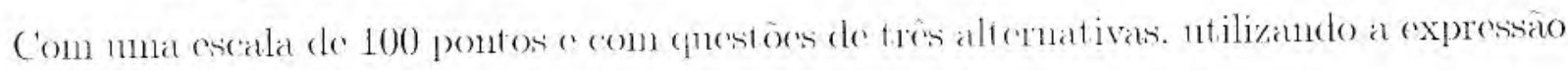


loganítnica. a pontuaço máxina á 24 a a míninna ó -76 . Portanto a distribuiçào da

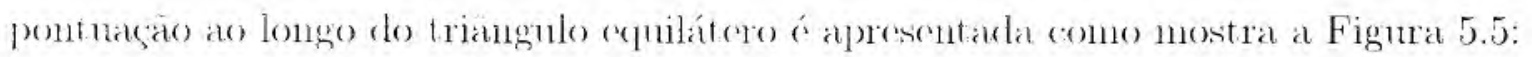

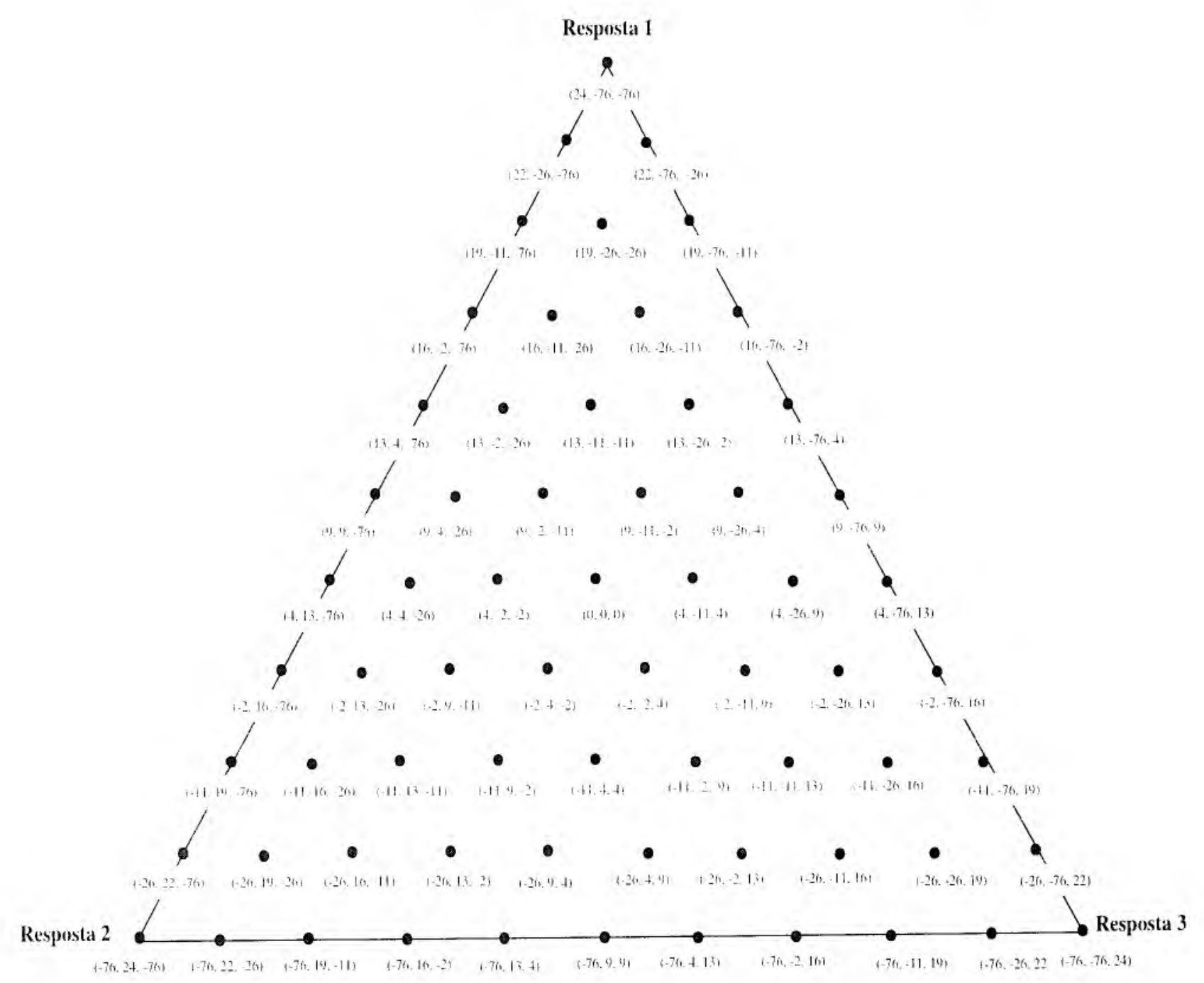

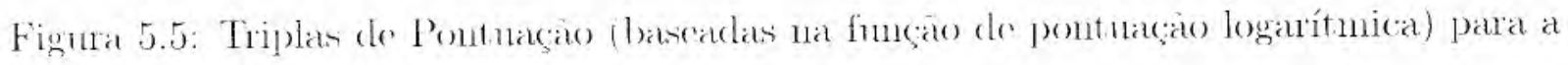

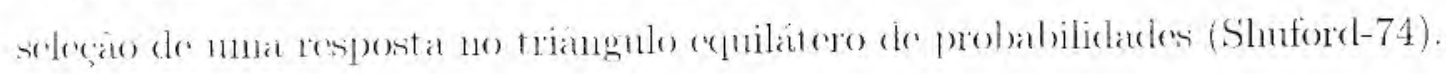

Nota-se que as penalidades tendem a ser maiores do que as recompensas para (gue a prática do "chute" seja inibida pois segundo Shuford \& Brown (1974), o estudante somente muda de comportamento quando a penaliclade associada a mma resposta incorreta i maior do che k-1 (k=munero de alt ernativas de resposta). Neste caso, o estndante tende a ser mais cantedoso a conn o pastsal de trinpo. quando faz mais testes com o método MPA. amadurece sem perisamento no sentido de exprestar sem conlecinento real através 


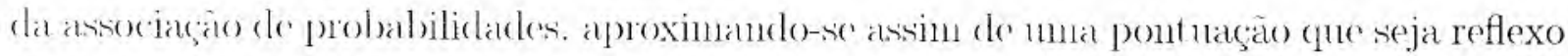
do sem estarlo de conlereinnento.

A Figma 5.6 compara duas medidas de informacion (roal e perecbida) para o primeiro teste com MPA realizarlo por 66 pessocols.

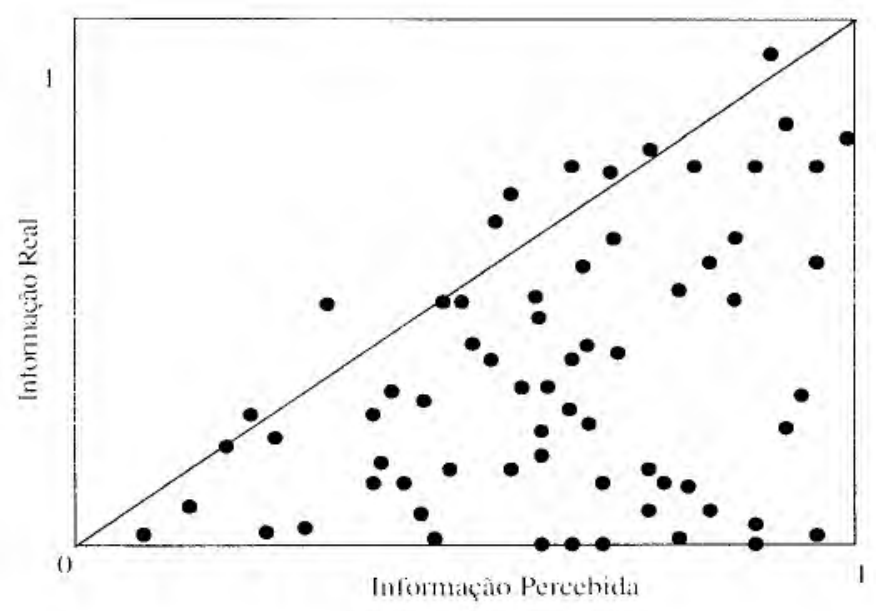

Figula 5.6: Comparaçato das informacoes (real e percebida) de 66 pessoas em $u$ momeiro teste administrado por compuntador com MPA (Shnford d Brown. 1974)

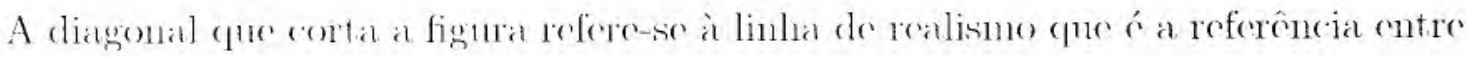

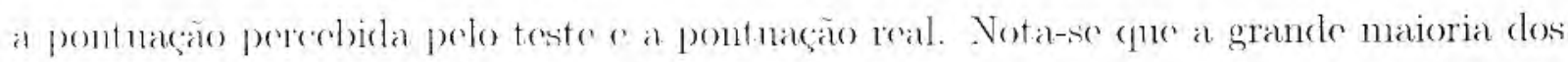
estudantes ustá localizada abaixo da diagonal, o cur significa mua supervalorização do

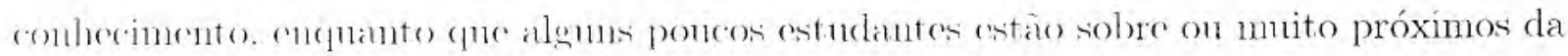
diagonal. representando acpuedes que comherem nuito benu sen estado de conhecimento.

\subsection{MPA adaptada por Bruno (1986), (1987), (1988)}

Bruno (1986) adapton o mótodo MPA para o conuputador aliminamedo alguns pontos de probabilidades intermediários. eonservander as caraterésticas gerais do método MPA. A

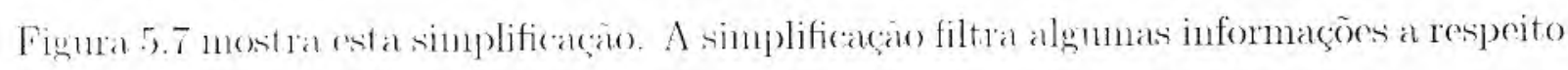

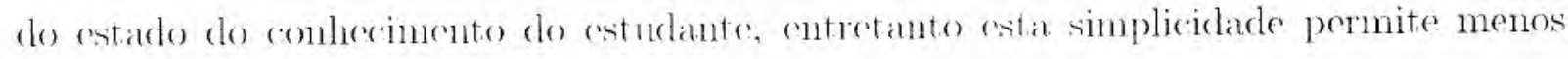

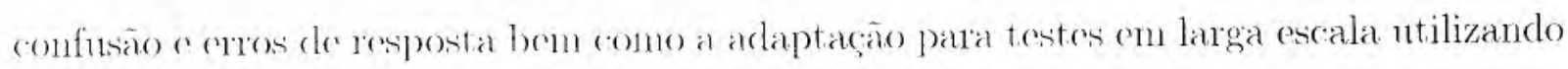

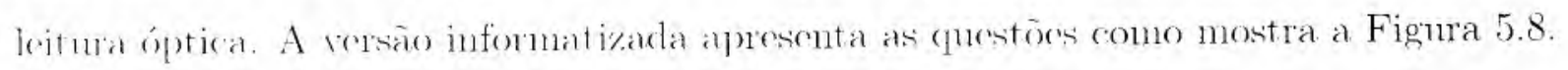




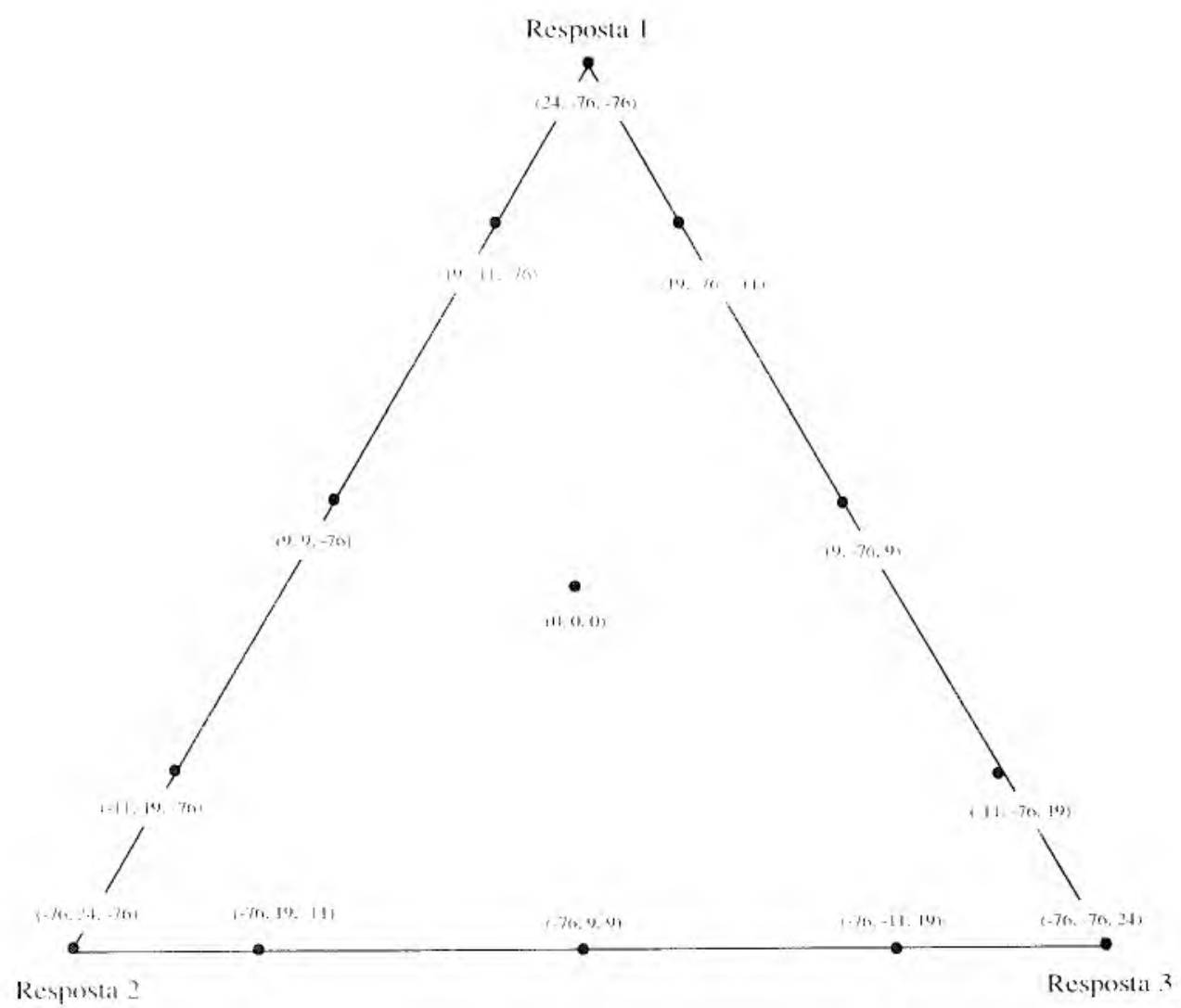

Figura 5.7: Estrumura simplificada para numa (juestão nsando MPA

() estudante responde conn a eseollat que representad a probabilidade subjetiva de

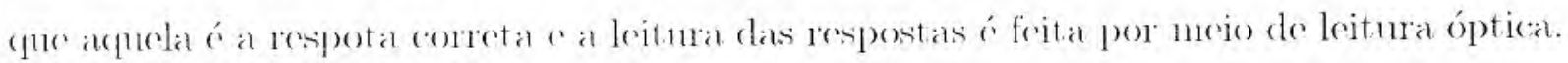

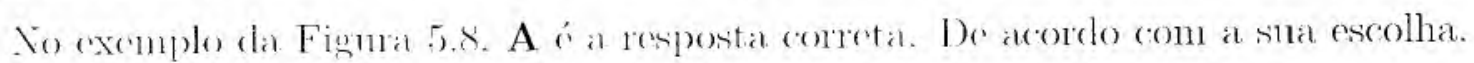

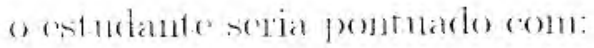

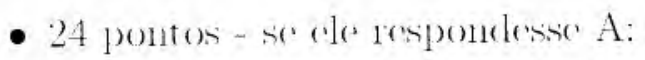

- -76 pontos - (aso respondesion B on C:

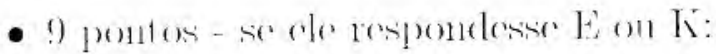

- 19 pontes - se ale respronelesse L on D;

- - 11 pontos - se ele respondenser J on F:

- () - se de respondense M: 


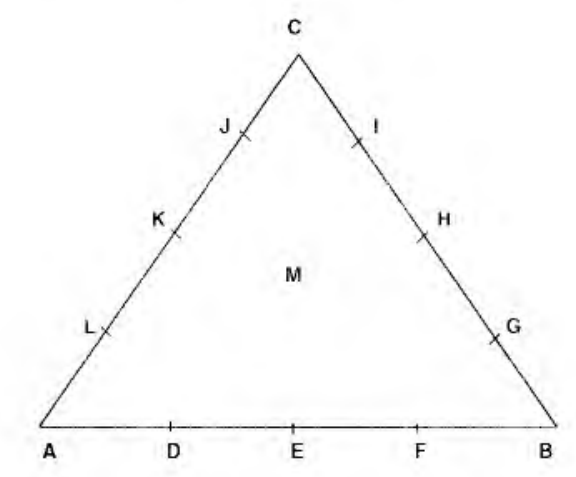

Questāo 1: Quem é o Presidente dos Estados Unidos

$$
\begin{aligned}
& \text { A. Reagan } \\
& \text { B. Bush } \\
& \text { C. Carter }
\end{aligned}
$$

Ou Use as letras de $D$ até $M$

Fignua 5.8: Exemplo de apresentaço em tela para nuna questão nsando MPA

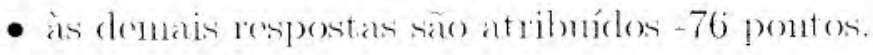

As penalidades orecompensas ansociadas à classificaçäo no MPA modificado de Bruno (1980) sáo mostradas na Tibela 5.1:

\begin{tabular}{|l|l|}
\hline Pontuação & Classificação \\
\hline 24 & Totalnuente Informarto \\
\hline 19 & (Muase Informado \\
\hline 9 & Parcialmente Informado \\
\hline 0 & Desinformado \\
\hline-11 & Parcialnuente Mal Informado \\
\hline-76 & Mal Informado \\
\hline
\end{tabular}

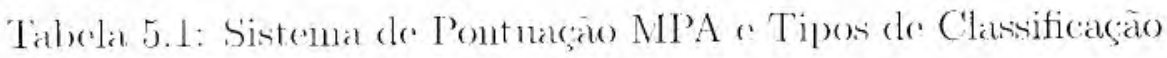

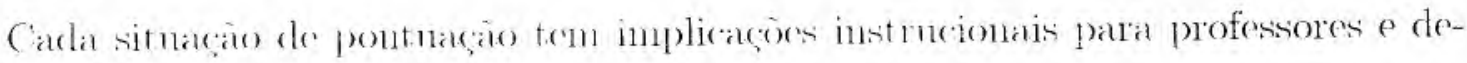
senvolverlones de sistrunas instrue ionais computadorizados (Brumo, 1987). A classificação propercionarla pelo método direciona as estratégias ligadas a cada classificação como mostrat a Tabela 5.2: 


\begin{tabular}{|c|c|c|}
\hline Classificação & Significado & Estratégia \\
\hline Totalunente Informato & Sabe a reposta & Avango \\
\hline (Quase Informado & & Avanço / Rrevisão \\
\hline l'arcialnemes Informato & Saleralgo dis respostat & Revisào \\
\hline Dersinformado & $\begin{array}{l}\text { Naso salue mada relativo à res- } \\
\text { posta }\end{array}$ & Instruçaio \\
\hline Parcialmente Mal Informado & & Instruçäo / Re-oducação \\
\hline Mral Informado & Nâle sabe a resposta & Rre-educaçãò \\
\hline
\end{tabular}

Tabela 5.2: Fstratónias reforentes as elassificacoes resultantes

Bruno (1986) relata $111 \mathrm{n}$ experinuento feito com un teste envolvendo 20 questoes.

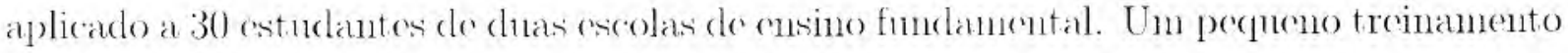

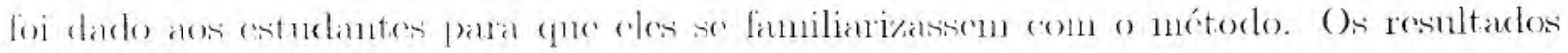

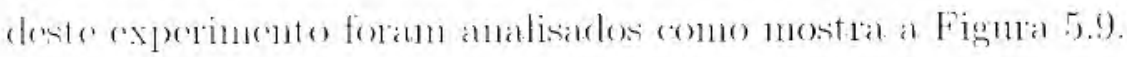

Resumo da analise de três estudantes

\begin{tabular}{|c|c|c|c|c|c|c|c|c|c|}
\hline \multirow[b]{2}{*}{ Tipo de informaçao } & \multicolumn{3}{|c|}{ Estudante A } & \multicolumn{3}{|c|}{ Estudante B } & \multicolumn{3}{|c|}{ Estudante $\mathrm{C}$} \\
\hline & $\mathrm{N}^{\circ}$ & $0_{0}$ & $\begin{array}{c}\text { Pont uaçào } \\
\text { MPA }\end{array}$ & $\mathrm{N}^{\circ}$ & $0_{0}$ & $\begin{array}{c}\text { Pont uaçà } \\
\text { MPA }\end{array}$ & $\mathrm{N}^{\circ}$ & $0_{0}$ & $\begin{array}{c}\text { Pontuaçāo } \\
\text { MPA }\end{array}$ \\
\hline Totalmente Informado & 20 & 100 & 180 & 0 & 0 & 0 & 2 & 10 & 48 \\
\hline Parcialmente Informado & 0 & 0 & 0 & 1 & 5 & 19 & 4 & 20 & 36 \\
\hline Desinformado & 0 & 0 & 0 & 18 & 90 & 0 & 7 & 35 & 0 \\
\hline Parcialmente Misl Informado & 0 & 0 & 0 & 0 & 0 & 0 & 0 & 0 & 0 \\
\hline Mal Intormado & 0 & 0 & 0 & 1 & 5 & -76 & 7 & 35 & -532 \\
\hline Pontuaçao MPA Total & & & 480 & & & -57 & & & -448 \\
\hline Fator de Ajustamento & & & 1520 & & & 1520 & & & 1520 \\
\hline Pontuaçao MIPA total ajustada & & & 2000 & & & 1463 & & & 1072 \\
\hline "o máxima & & & 100 & & & 73 & & & 54 \\
\hline Número de respostas corretas & & & 20 & & & 1 & & & 4 \\
\hline$\%$ conteta & & & 100 & & & 5 & & & 20 \\
\hline
\end{tabular}

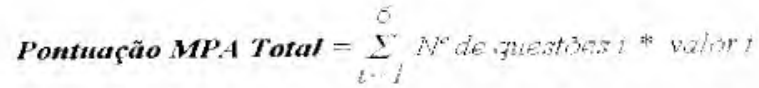

Fator de Ajustamento $=N^{N}$ de duestrie: ${ }^{\text {* }} 76$ (ermu todas $)$

Pontuação MPA total ajustada = Pontuaçao MPA /otal + Fator de Ajustamento
}

Figua 5.9: Análise de resullandos- Bromo-86

Na Fignara 5.9. é apresentado o desempenho de tres estudantes mostrando na pri-

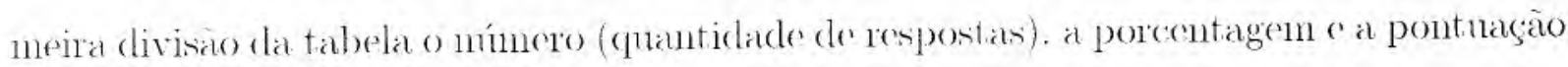




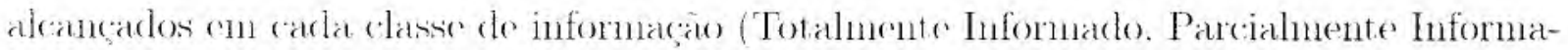
(lo. Desinformado, Mal Informado. Pareialmente Mal Informade). Na segunda divisào da

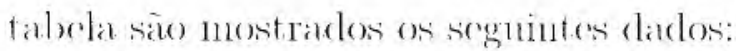

Pontuaça MPA Total: soma da pontuacão MPA por classo de informação;

Fator de Ajustamento: número de chestón da prova * 76 :

Pontuaçà .MPA Total Ajustada: Pontuação MPA Total + Fator de Ajustamento;

\%/: Máxima: porcentagem de pontuaçào MPA Total a justada da pontıação máxima:

Número de resposas cometas: quantidade de acertos:

\% de Cometas: porcentagen de respostas corretats do total de respostas.

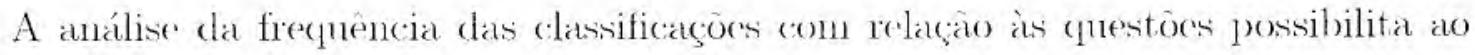

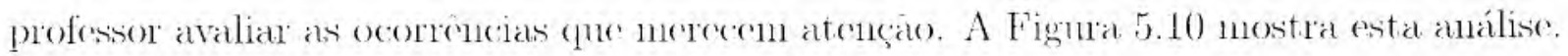

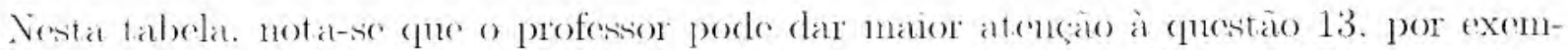

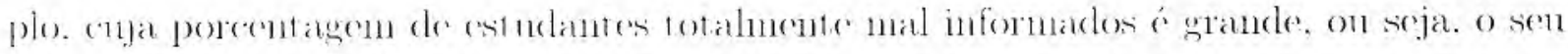

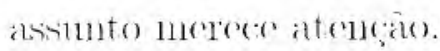

Análise das questöes: Freqüência

\begin{tabular}{|c|c|c|c|c|c|}
\hline Questäo & $\begin{array}{l}\text { Totalmente } \\
\text { Informado }\end{array}$ & $\begin{array}{l}\text { Parcialmente } \\
\text { Informado }\end{array}$ & Desinf ormado & Mal Informado & $\begin{array}{l}\text { Parcialmente } \\
\text { Mal Informado }\end{array}$ \\
\hline 1 & 710 & 9.7 & 6.5 & 65 & 65 \\
\hline 2 & 306 & 32 & 65 & 0.0 & 97 \\
\hline 3 & 903 & 32 & 32 & 00 & 32 \\
\hline 4 & 710 & 00 & 258 & 32 & 0.0 \\
\hline 5 & 452 & 129 & 22.6 & 32 & 161 \\
\hline 6 & 129 & 16.1 & 484 & 3.2 & 19.4 \\
\hline 7 & 16.1 & 355 & 25.8 & 6.5 & 16.1 \\
\hline 8 & 58.1 & 9.7 & 129 & 00 & 194 \\
\hline 9 & 806 & 3.2 & 16.1 & 00 & 00 \\
\hline 10 & 871 & 32 & 3.2 & 00 & 65 \\
\hline 11 & 710 & 65 & 12.9 & 00 & 97 \\
\hline 12 & 613 & 12.9 & 12.9 & 6.5 & 65 \\
\hline 13 & 290 & 194 & 161 & 6.5 & 29.0 \\
\hline 14 & 419 & 97 & 323 & 00 & 16.1 \\
\hline 15 & 19.4 & 258 & 323 & 6.5 & 16.1 \\
\hline 16 & 581 & 00 & 161 & 00 & 258 \\
\hline 17 & 419 & 12.9 & 35.5 & 00 & 97 \\
\hline 18 & 645 & 6.5 & 226 & 32 & 32 \\
\hline 19 & 38.7 & 16.1 & 194 & 00 & 158 \\
\hline 20 & 774 & 32 & 16.1 & 0.0 & 32 \\
\hline
\end{tabular}

Fignua 5.10: Análise de resultados - Broune-86 


\subsubsection{MPA com escala modificada-Confidence Weighted-Admissible Probability Measurement (MCW-APM)}

Brmo (1988) relata mana MPA modificadia. chamada de MCW-APM. em um teste com pontuaça pré-estabelecida (CRM). para diagnosticar o estado do conhecimento dos es-

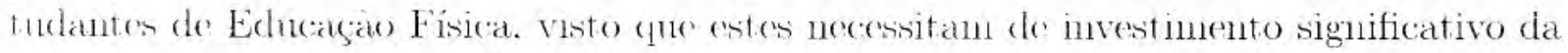

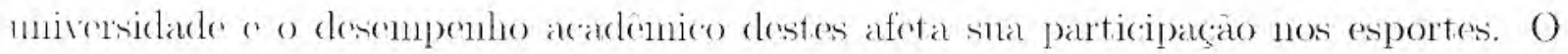
suporte acaulemine para estes estudantes ce nunito inportante pois cles chegann do colégio

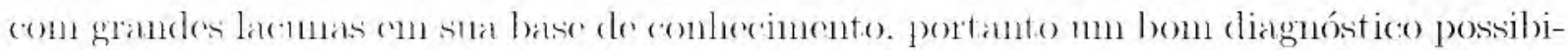

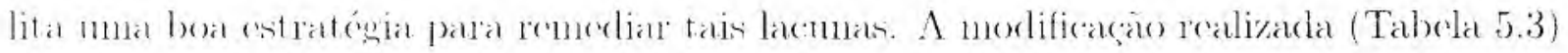

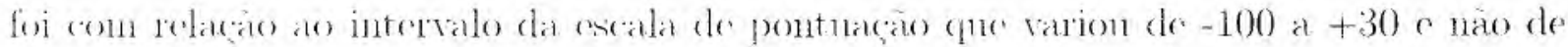

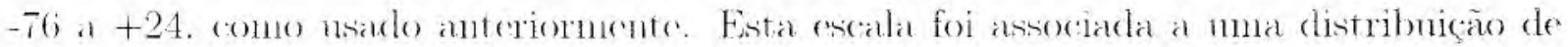
porcentagen mais tradicional $(100.75 .50 .25,0)$ referente a $1 m$ peso de confiança na resposta.

\begin{tabular}{|l|l|l|}
\hline Classificação & Porcentagem & Pontuação \\
\hline Totalnnente Informado & 1.00 & 30 \\
\hline Qnaso Informado & 75 & 20 \\
\hline Parcialmento Informado & 50 & 10 \\
\hline Desinformado & 33 & 0 \\
\hline Parcialnuente Mal Informado & 25 & -10 \\
\hline Mal Luformatolo & 0 & -100 \\
\hline
\end{tabular}

Tabela 5.3: Escala de pontinaçào modificada por Bruno-88

Brumo (1988) stgere muna análise de resultados dividiela en tres partes. A primeira.

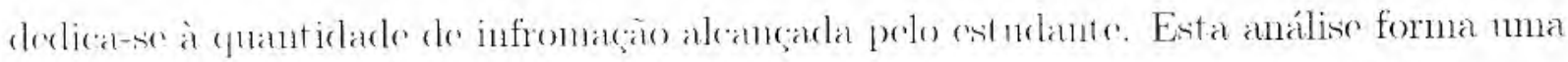

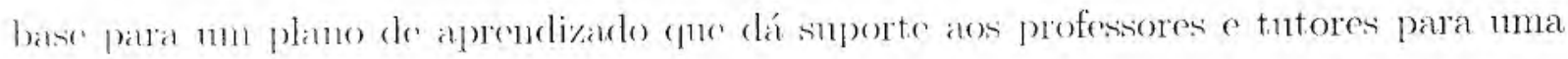
instruço mais individualizada. A segunda parte. é nma avaliação curricular baseada na reTteza on incerteza de 1111 estuddante quanto à informacão. É mua comparação de todas as questöes por assimto do teste por moio das porcentagens e frequências alcançadas por um ostuclante. Por meio desta análise o professor identifica quais assuntos merecem atenção aperial derido anos indicativos elevados de estudiuntes mal informados on desinformados. 


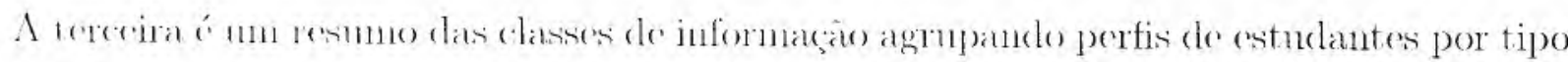
de information.

\subsubsection{Análise dos resultados de um teste baseado na MPA para um estudante considerado fraco}

A seguir á mostrado o plano de aprendizado para un estudante considerado fraco (Tabe1.15.4).

Pontuação MPA: 0.81

\begin{tabular}{|c|c|}
\hline Escala & Classificação - Estratégia \\
\hline $95-100$ & Totahnente informado (bon conhecineme) - avançar \\
\hline $85-94$ & Bem informatlo (couleceinento razoável) - uecessário revisar \\
\hline $76-81$ & Fratemente informaklo (conlereinente) fracos)- instruir \\
\hline$(5)-75$. & 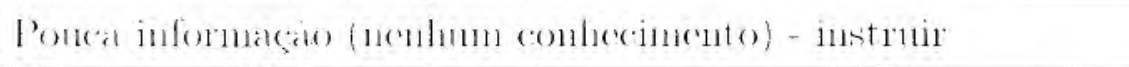 \\
\hline () -64 & 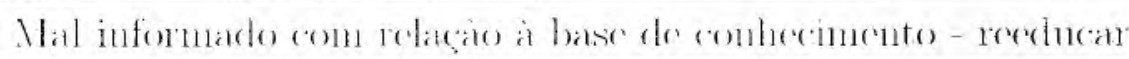 \\
\hline
\end{tabular}

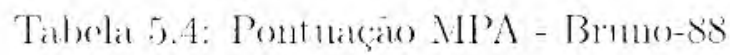

\section{Pontuação sobre o uso da informação: 0.87}

Porentagen de vezes em gue o estudante estava correto quando utilizon uma das alteruativas A, B on C (Tabela 5.5).

\begin{tabular}{|c|c|}
\hline Escala & Classificação \\
\hline 9()$-10()$ & Bontu 1tiso da informacaro \\
\hline$\wedge()-89$ & 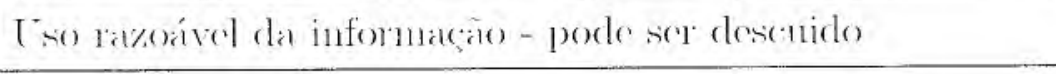 \\
\hline$(10)-79$ & Ponco nso da informaçäe - descuide no nso da informação \\
\hline
\end{tabular}

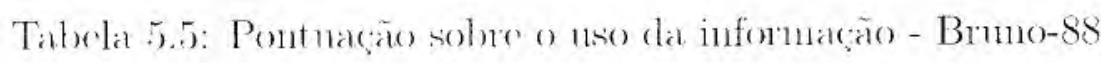

Porcentagem geral de certeza na prova: 0.50

Porcentagenu de respostias A. B. C'ntilizadias na prova do total de possíveis respostas (Tialorlit 5.6). 


\begin{tabular}{|c|c|}
\hline Escala & Classificação \\
\hline 8()$-100$ & Boa confianega total has lespostas \\
\hline 5()$-79$ & Fraca confiancat total nats respostatis \\
\hline$(0)-50$ & Poncar confianca total nas respostato \\
\hline
\end{tabular}

Tabela 5.6: Porentagem geral de certegat nat prova - Brumo-88

\section{Porcentagem de certezas corretas na prova: 0.41}

Poreentagen de respostas A. B.C utilizadas na prova que estavam corretas do total do possiveis respostas (Tabela 5.7).

\begin{tabular}{|c|c|}
\hline Escala & Classificação \\
\hline 8()$-100$ & 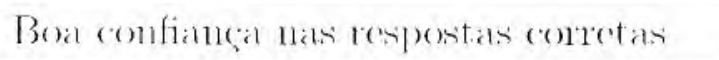 \\
\hline 5()$-79$ & 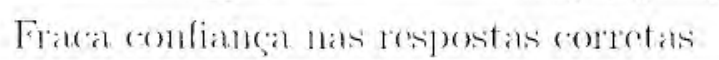 \\
\hline$(00-50$ & Ponca confiauna nas respostas corretats \\
\hline
\end{tabular}

Tatrelar 5.7: Porechitagem geral de certera na prova-Bromo-88

\section{Porcentagem de incerteza: 0.50}

Porcentagem de respontats D, E. F, G, H. I. J. K. L. M do total de possíveis mespostas (Tabelat 5.8).

\begin{tabular}{|c|c|}
\hline Escala & Classificação \\
\hline $00-10$ & Incertezal normal cuu nivel cscolar \\
\hline $10-20$ & Incerteya acinua do normal em nível escolar \\
\hline $21-100$ & Mnita incertezad - exame foge do nivel escolar \\
\hline
\end{tabular}

Tabela 5.8: Porcentagem geral de certezat na prova - Bruno-88

\section{Porcentagem de falta de informação}

Porentagen de respostas . M on respostas deixadas em braueo do total de respostas posiveris. 


\begin{tabular}{|c|c|}
\hline Esciala & Classificação \\
\hline 0()$-10$ & falla de informatgate normal em nível escolat \\
\hline $10-20$ & 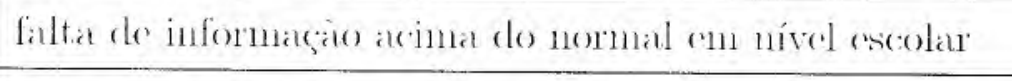 \\
\hline $21-100$ & muita falta de informatgio - exame foge do nivel esceolan \\
\hline
\end{tabular}

Tabela 5.9: P'orcentagem geral de certerat mal prova- Bruno-88

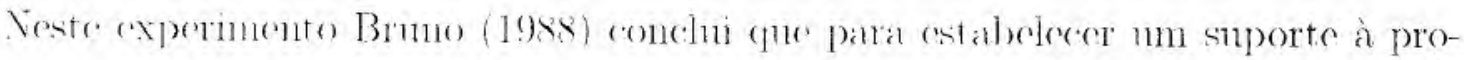

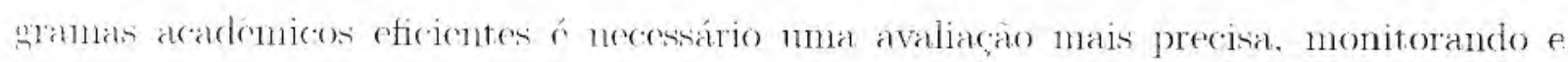
diagnosticando cono está a base de conlecimento de run estudante.

\subsection{MPA utilizada por Klinger (1997)}

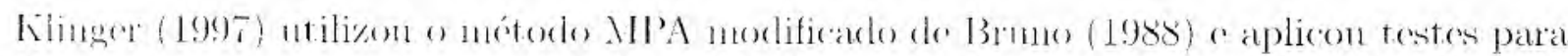

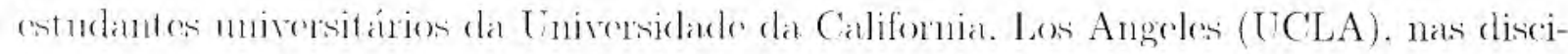

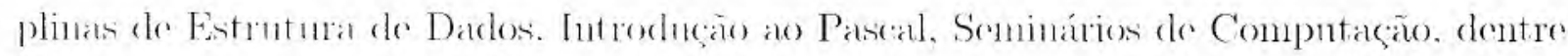
ontras. (om poutnaçào comparativa (NRM).

Va análise de resultados. Kilinger (1997) referencia a relacào entre acertos erros.

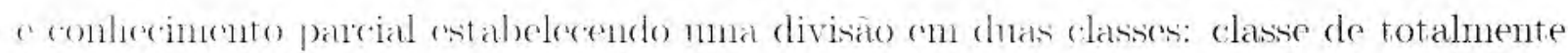
informato + quass informade c (tasise de mal informado + desinformado. Esta forma possibilita vistlalizar estudantes que ficann no limian entre as classese, ficando a cargo do professor a estratégia a ser tomada frente a essere resultade.

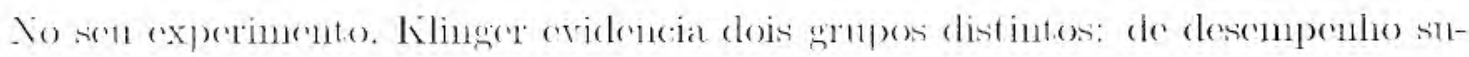

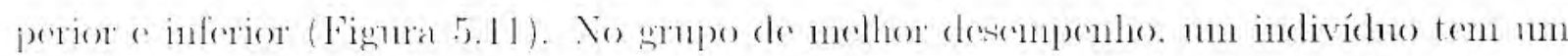

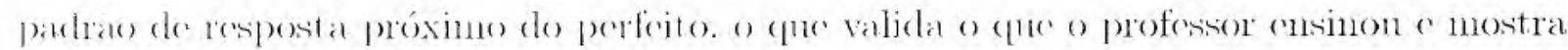

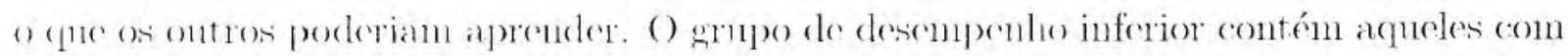
alguma falta de entendimento. sendo os nraiores responsíveis pelas perguntas, interaçoes

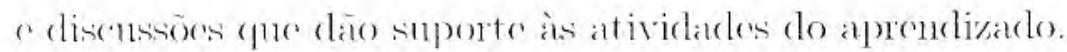

Na taluela da Figura 5.11. o cixo W+B refere-se a culantidade de respostas erradas

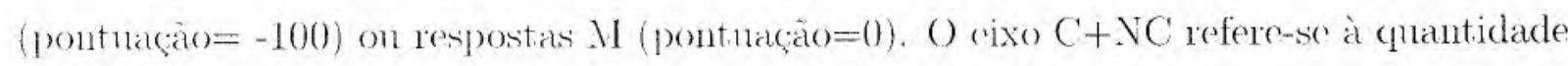

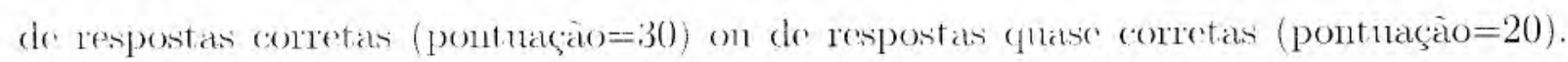
() intervalo tanto do cixo $\mathrm{W}+\mathrm{B}(\mathrm{de} 0$ a 12 ) quanto do cixo $\mathrm{C}+\mathrm{NC}$ (de 17 a 28 ) refere- 


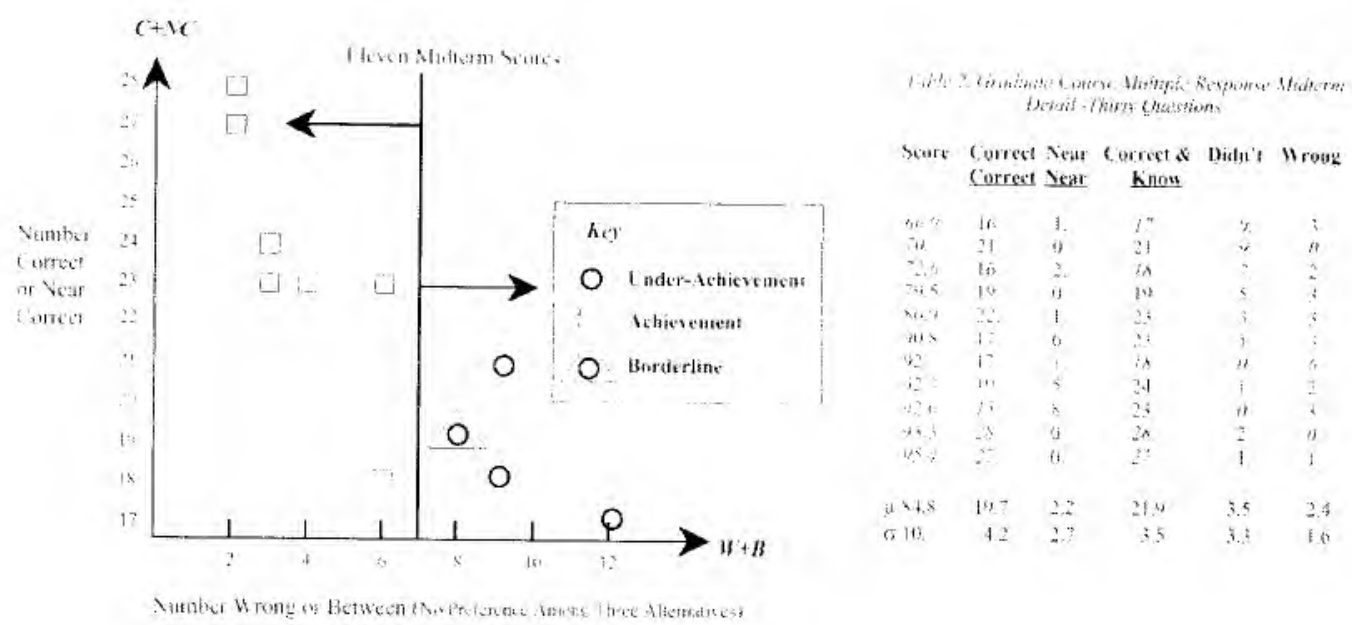

Fignar 5.11: Análion do resultades - Kilnger-97

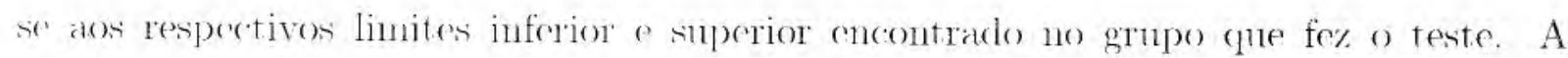
linha vertical que cortia o gráfico. delimitando as classificarcoes, refere-se' ao valor de 0.769 $01176.9 \%$ que o o valor que incliea a falta de conhecinente do estudante. on seja, se

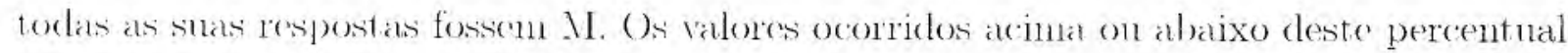

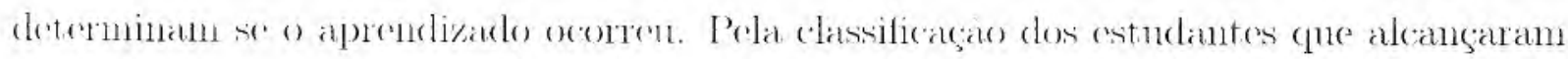

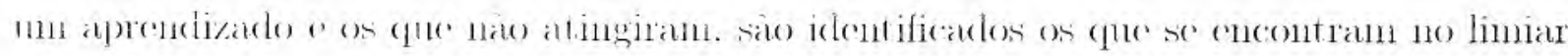

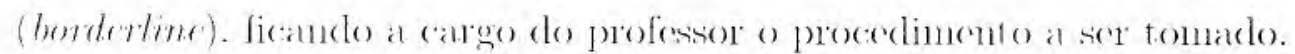

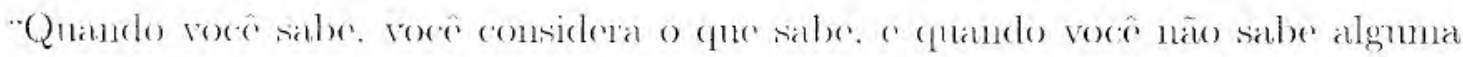

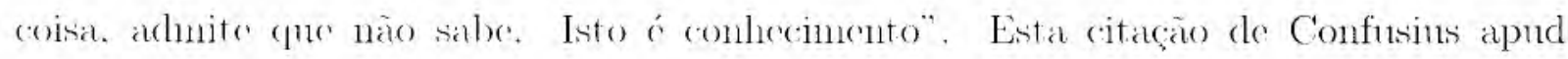
Shutord d Browu (1974) a rempeito do verdadeiro conlecimento estimula o desejo de se

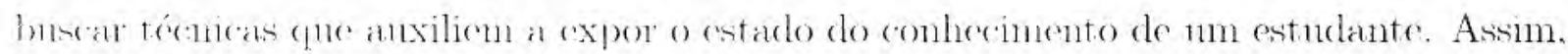
MPA colabora com esta visăo de avaliacion, encolajando a associar a tecnologia com sna prática. de forma que as necessidades de testes em larga escala scjam atendidas sem prejudicar a qualidade de muna avaliaçào. 


\section{Capítulo 6}

\section{Avaliação dos Exames de}

\section{Proficiência em Inglês Desenvolvidos}

Atro o início de 2000. os estndantes (pue ingressavam no prograna de nestrado do ICMCUSP' rran avaliados com rolagio à proficiencia cun ingles por meio de exanes preparados a

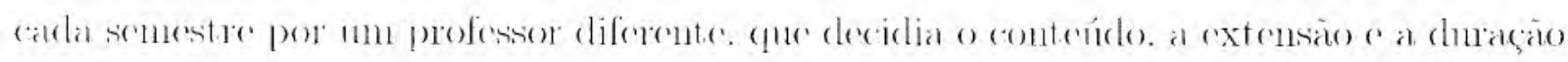

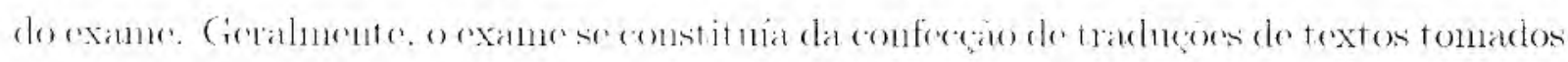

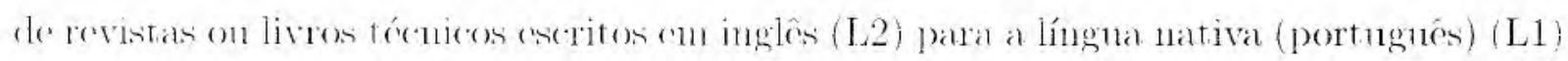

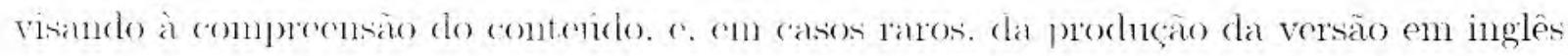
de ontro texto de literatura técnica. originariamente na língua nativa. on da produção de $1 u$ texto on inglós de autoria própria.

A corrector dos exames de proficiencia era colstosa e comsumia bastante tempo

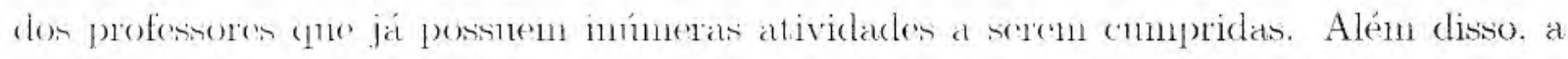

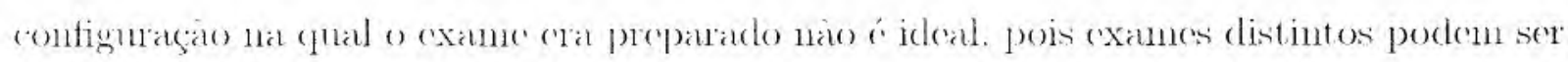
fortenente nàro miformes devide à rotatividade de professores responsáveis. e também à

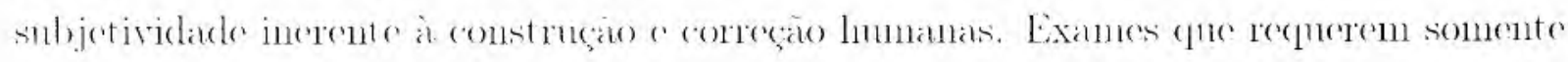

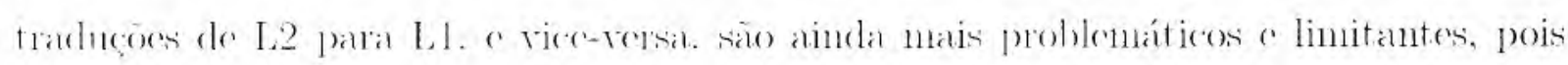

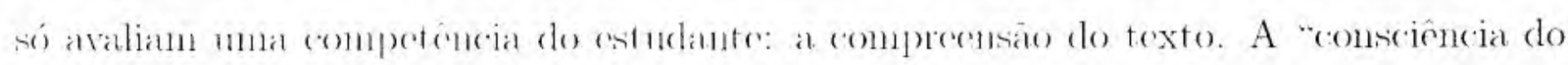

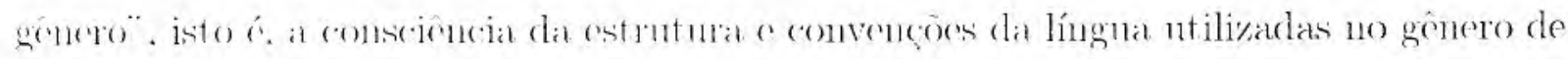
fexto autigo (iontifico. (onno (xppressa Swales (1990)). năo ó incentivada. o que é essencial patra o pescuisador novato desempenhar molhor e mais rápiclo as tarefas de leitura e escrita 


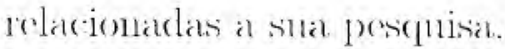

Lina opgào para este cenário poderia ser a exigencia do TOEFL (http://www.toeflorg)

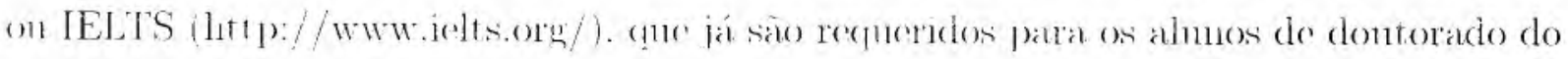
ICMC-TSP. também para os alunos de mostrado. Apesar destes exames serem bastante

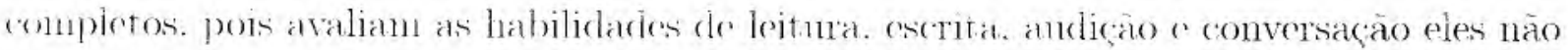
tratan de $14 m$ dos objetos mais importante para nu pescunisador: o artigo científico. Exames como o TOEFL exigem ma habilidade extra do alıno: rapidez na resposta, característica que gera a necessidade do aluno se preparar para passar neste tipo de exame.

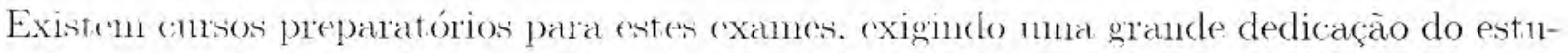
- Wante ate atotal innersào nesta atividade. fazendo conn que ele pare on rediza o tempo

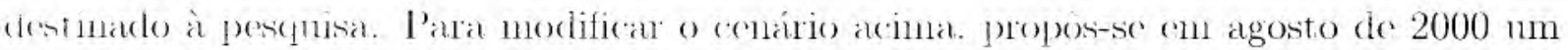
novo tipo do Exame do Proficiencia ('m Ingles (EPI) para o mestrado do ICMC que além de avaliar a eomprecusaio do texto. avalia a habilidade de reconherer o gênero de textos

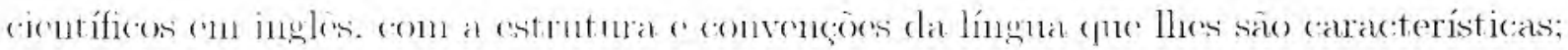

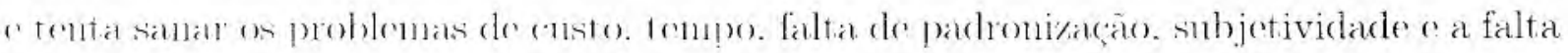

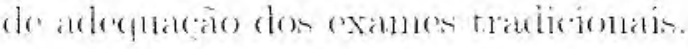

Para se chegar ao Exame Automatizado de Proficiencia em Inglês realizado em Abril de 2001. foram adquiridas experiencias por meio dos exames pilotos e formais (informatizados e no formato lápis-panjel) entre 1998 e 2001. (tilizon-se tanto nsuários reais estudantes de mestrade que realizaram os exames formads de proficiencia em inglês do ICMC-LSP) quanto colaboradores, 110 intuito de identilicar aspectos relativos à antoma-

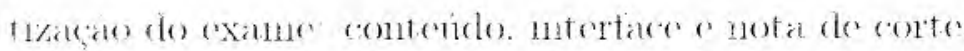

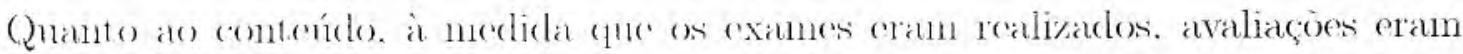

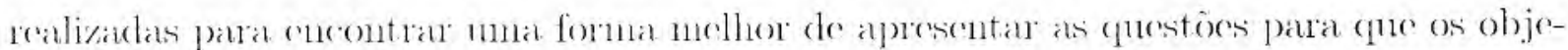

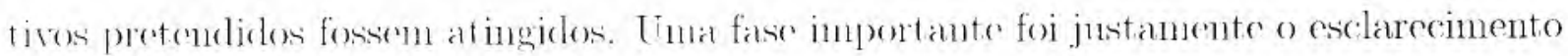

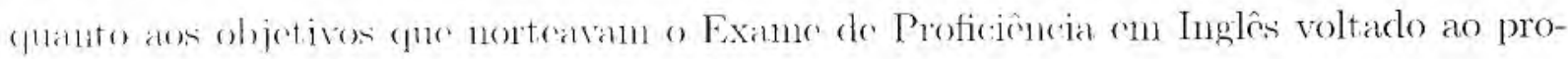
grama de mestrado. Con o apoio da Taxonomia do Bloom apresentada no Capítulo 4. alcancon-sc uma melhor especificaço dos objetivos e. consecpüentemente. mana melhora considerável na maneira de expressisar as questöes. além de ma prova mais equilibrada em tormos de habilidades a serem avaliadats. A primeira versiano do exane (proposta en agosto 


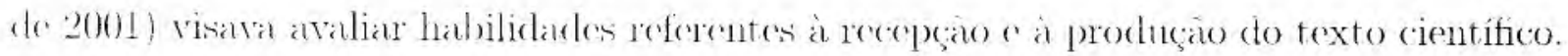

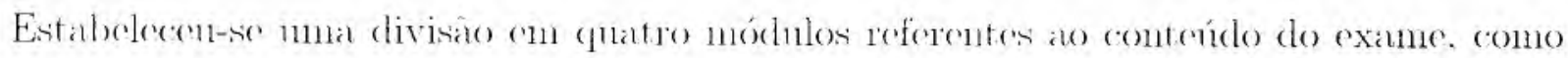
montrat a Tabelat 6.1.

\section{Módulo 1: Convenções da Língua Inglesa para Textos Científicos}

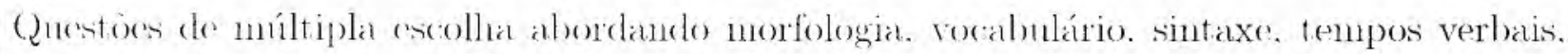

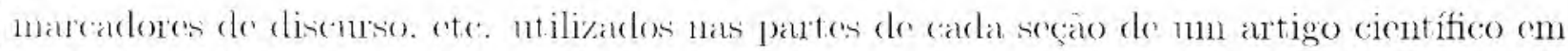
ingles.

\section{Módulo : Estrutura de Textos Científicos}

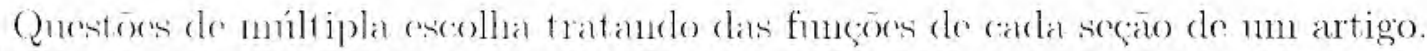

\section{Módulo 3: Operações de Sumarização}

Questóses de múltipla escolla tratando das cstratégias de resimo de $u m$ texto.

\section{Módulo 4: Estratégias de Escrita}

Questoes de multipla escolha abordaudo estratégias de escrita como. por exemplo, definiçoes. descriçoos. classificagoes. argunnentaçoes.

Taluela 6.1: Prinerira versão do EPI (Agosto de 2000)

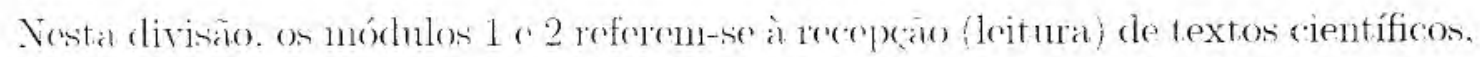

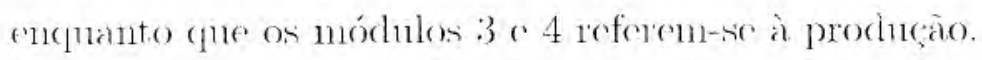

Enu termos do habilidades avaliadas a classificacão dos módulos de acordo com a Taxomomia de Bloom é mostrada na Tabela 6.2 :

\begin{tabular}{|c|c|}
\hline Módulos & Classe da Taxonomia \\
\hline M1 Convençốs da Lingua Inglesa para Textos Científicos & Conhecimento \\
\hline M2 Estrutura de Textos Cientificos & Allálise \\
\hline M3 Operaçós de Simnarizaçio & Avaliação \\
\hline M4 Listratégias de Escrita & Compremsão \\
\hline
\end{tabular}

Tabola 6.2: Classificacão dos módulos com base na Taxonomia de Bloom

De nuna format mais esperificat. o) módulo $M \perp$ pertence à suldelivisão "Conhecinento

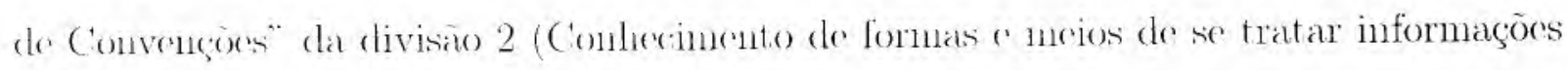




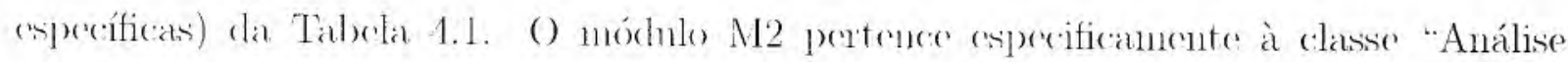

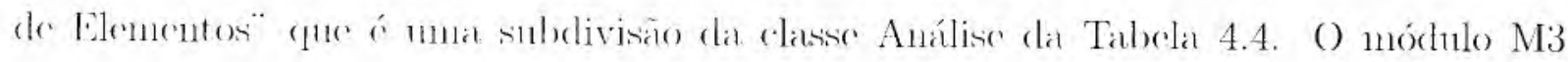

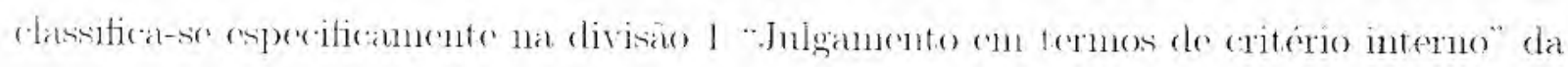

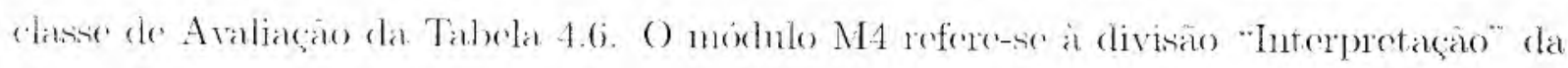

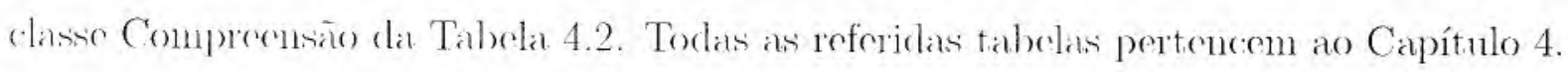

Para responcler as cuestöes do módulo M1. o processo mental envolvido é a recuperaçâ de convençõos da língua: para o módulo M2. o aluno deve quebrar o texto nos elmentos constituintes da Estrutura fornecida; no caso do módulo M3, una avaliação da possibilidade de trechos serene retirados sem que se perea nuformaço fundamental e fluxo

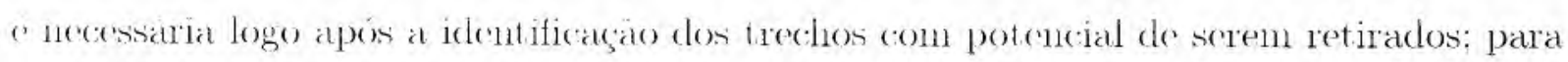
o módhlo M4 basta a leitura "interpretacaio dos trechos de texto para que os mesmos sejann associados dis respectivas classificacoes.

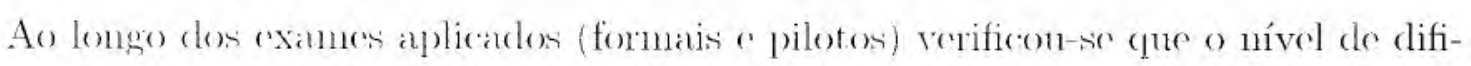

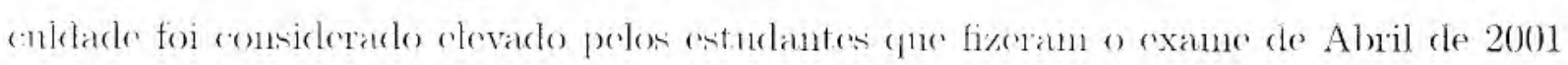

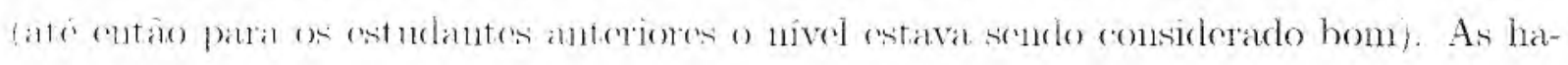

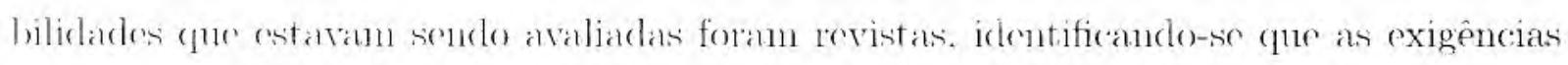
para 1 me estudante de mestrado do programa do ICMC-LSP estavam sendo superiores ao necessário. pois estavam sendo exigidas habilidades referentes à produção de textos (rentificos. Além disso. o fato das questòes estarem em inglês também colaboron para derate onvel dos testen

Juntanente conn o conterirto do exame buscont-se avalian o processo de antoma-

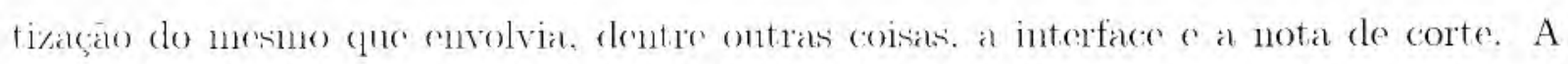

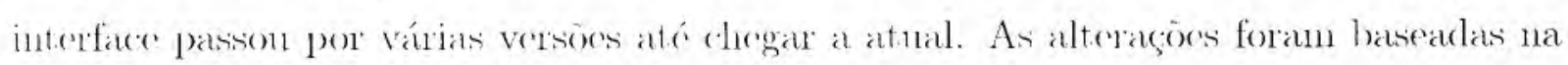
avaliaçäe de usabilidade gue será descrita na Segäo 6.6 .

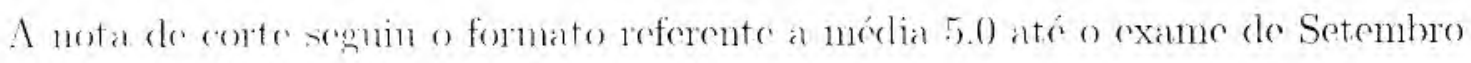

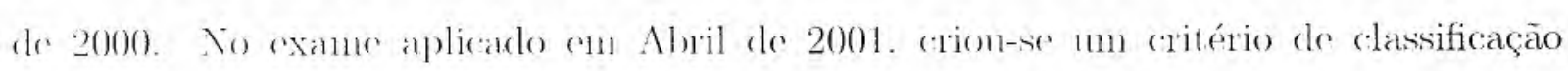
bascado em alguns aspectos da abordagenn de Klinger (1997) que utiliza a relação entre acertos. erros. c conhecimento parcial estabelecendo una divisão em duas classes: classe totalnente informados + (plase informados e mal informados + desinformados como visto na Seção 5.3. do Capítulo 5. 


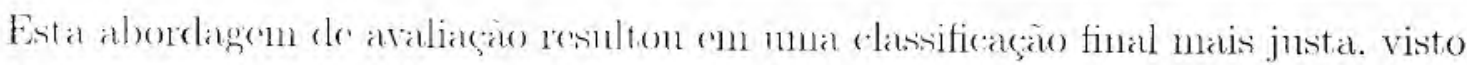

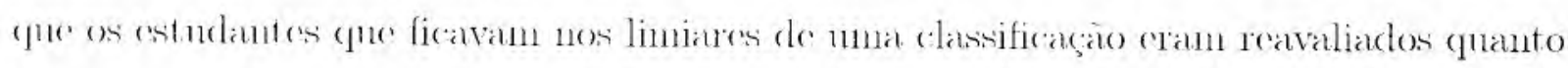

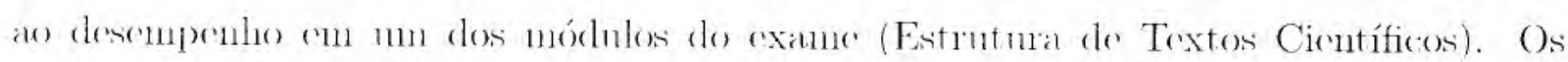

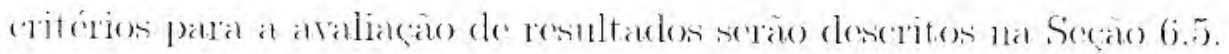

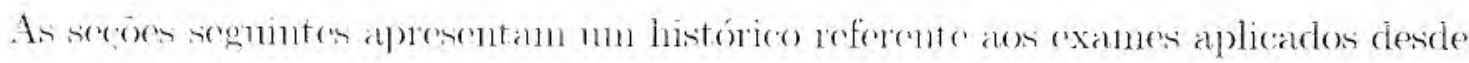
1998. abordando aspectos gerais o as licoes apreudidas en carla cxame até culminar na claboracão do site do sistema Computer Assisted Proficience Test of English for Academic Purposes (CAP'TEAP). a ser ntilizado para os EPIs do programa de mestrado do ICMCLSP.

\subsection{Exame formal realizado em Abril de 1998: Mu- dança de enfoque do conteúdo do EPI}

\section{Aspectos Gerais}

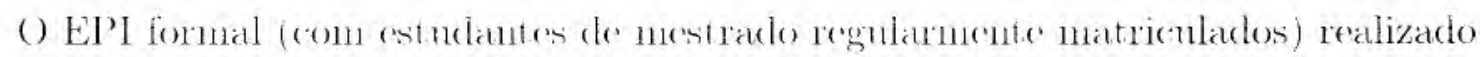
rom 29/04/1998 pela Profa. Dra. Sandra Maria Alnísio fol aplicaulo para as áreas de

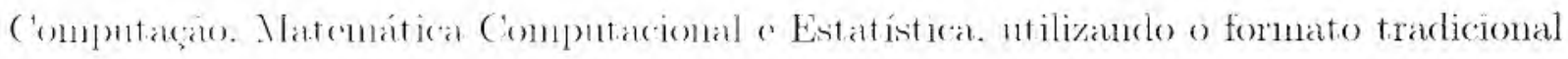

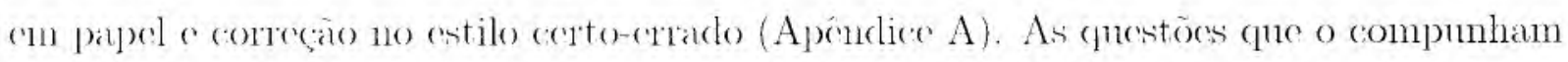

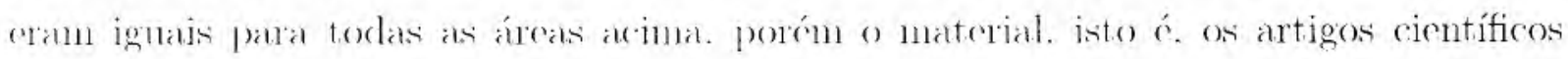

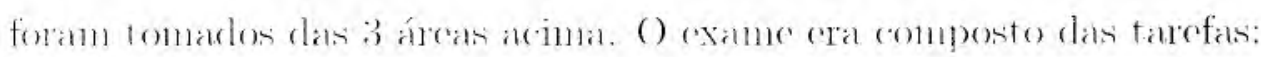

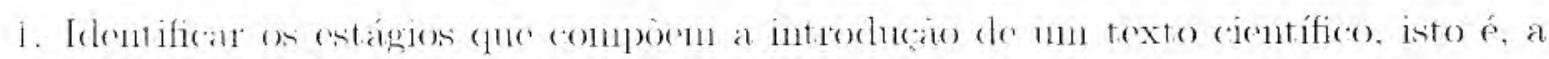
astrutura do muna introducion:

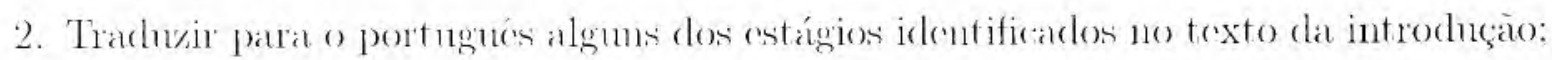

3. Escrever 1 m resimno en ingles sobre 1 mu trabalho realizado on da pesquisa de mestrado. segninde) nuna cestrubtura típ)icer. fornecida au estudante.

\section{Experiência adquirida pela análise dos resultados}

() intnito principal da aplicação deste exame era a avaliação da mudança de enfo(fue do conterído. diferenciando-se dos anos anteriores gute na maioria das vezes utilizava principalnente a traducaio. Os resultades obticlos forane encorajadores pois os estudantes 


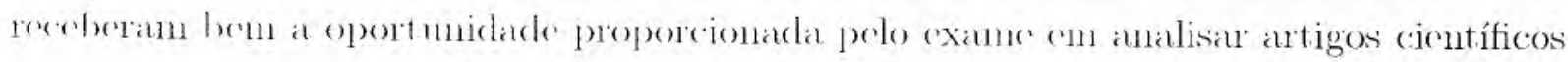

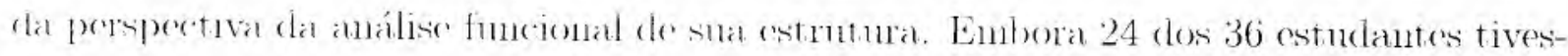

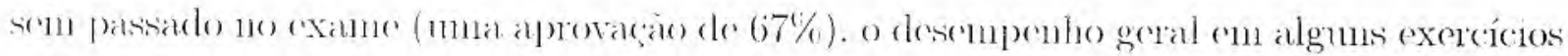

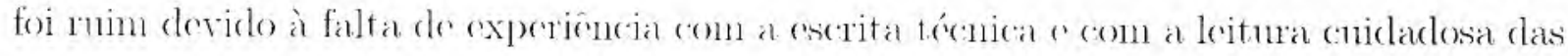

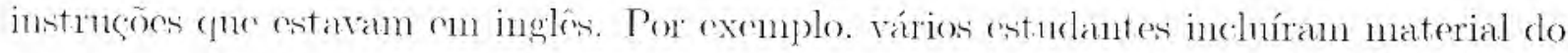
abstract na tarefa 1. embera as instrucoós para se tratal somente a estrutura da Introducato temliam sido claras. ()s estudantes tambén falharam em reconhecer algims componentes da estrutura do texto da introdıçào fornecido. o fure ocasionon a identificação de sconnentos mais longos do que o csperado, on seja, ao contrário de mais subdivisōes com parágrafos curtos apresentando matis componentes da introduçádo. houve ponca sub)-

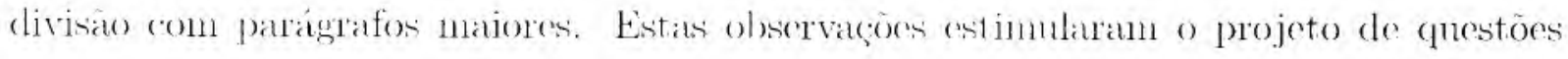

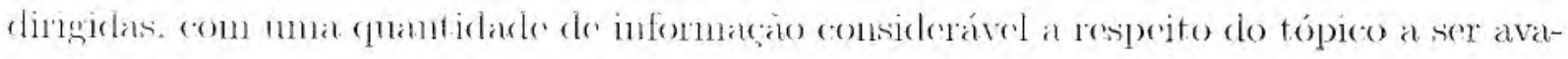

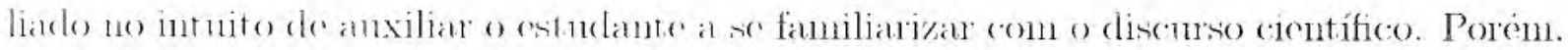

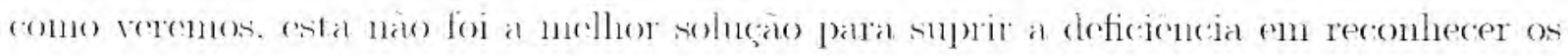
componentes funcionais de artigos.

\subsection{Exame piloto realizado em Setembro 1999: Mé- todo automatizado tradicional (certo-errado) no formato lápis e papel}

\section{Aspectos Gerais}

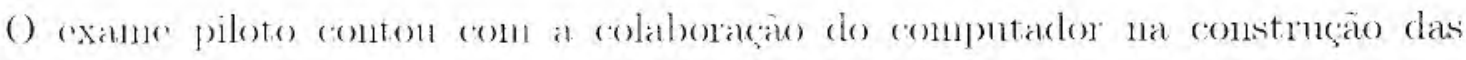

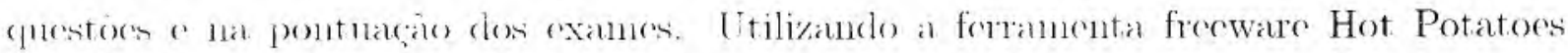

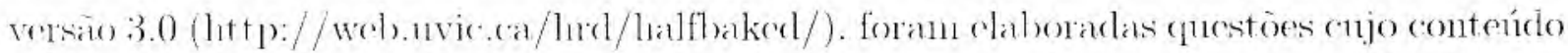
cutava voltado at textos científicos, hascando-se na experiencia adquirida no EPI realizado on Abril de 1998. Foram utilizados cinco tipos de questòes clos seis que a ferramentar possibilitava criar, como mostrà a Fignra 6.1. As questões (Apêndice B) foram impressias para serem aplicadas a $1 m$ grmpo de colaboradores composto por estudantes

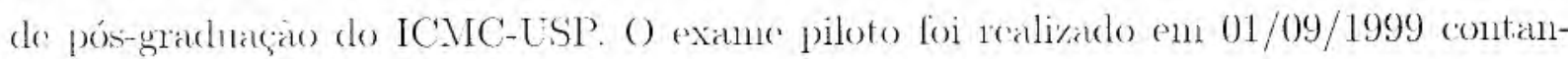
do com a participaçào de 10 pós-graduaudos da área cte computação. As questóns fo- 


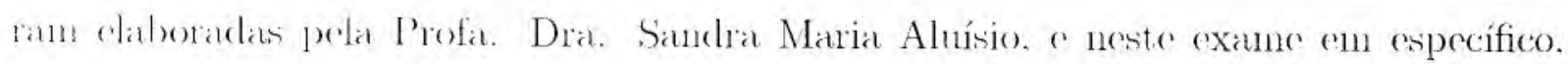
ntilizaram-se textos da Físiea retirarles de mun das ferranentas do anbiente AMADEUS

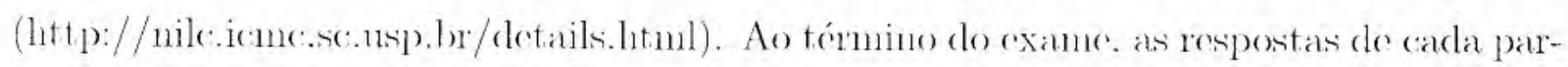

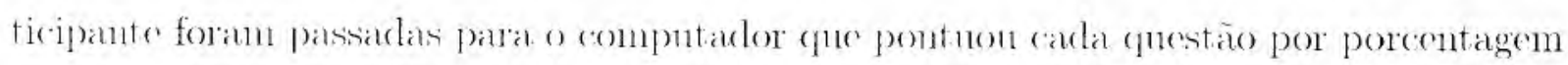
de acerto. Näo foi utilizado o computador pois o Hot Potaoes gera exames en Java Seript que deixa o resultado das questóses atrelado ao texto html.

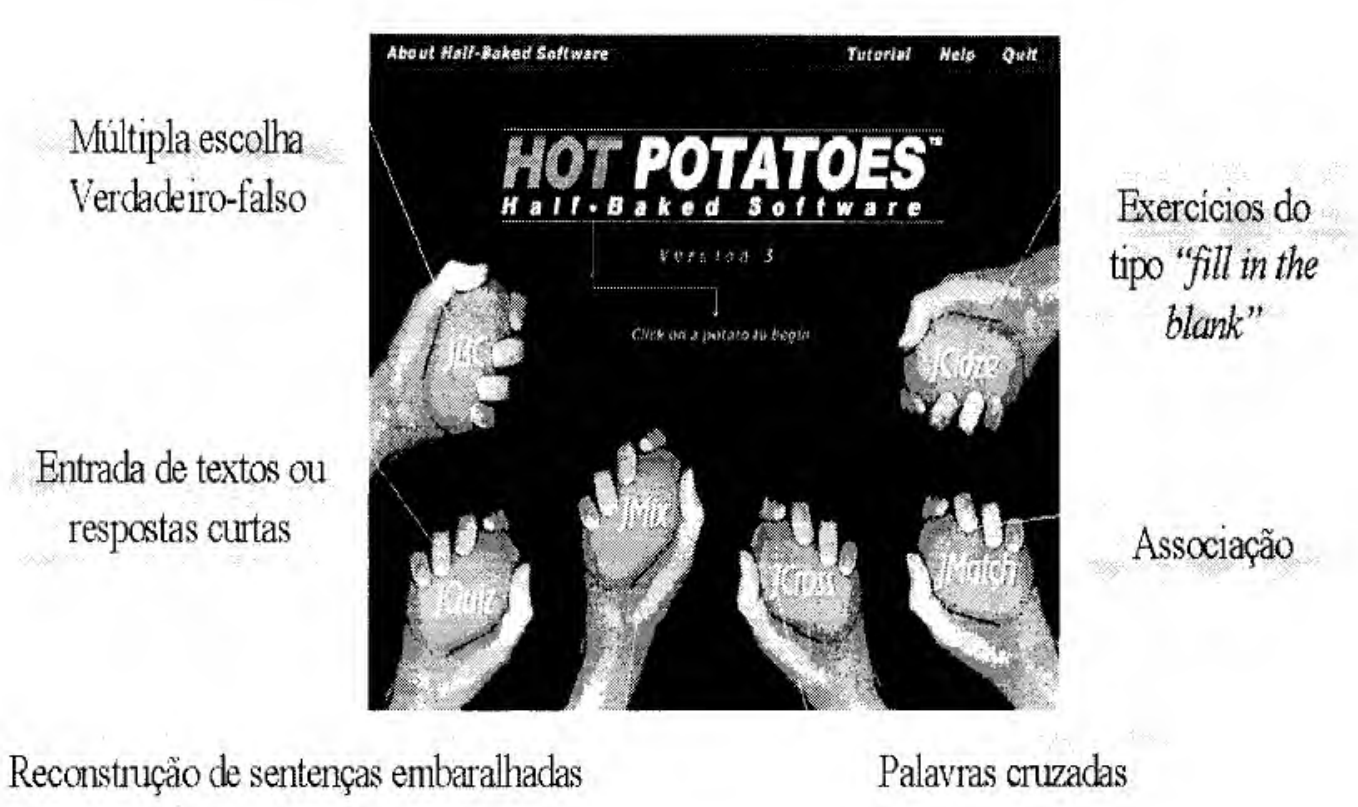

Figura 6.1: Tipos de curestoos da ferramenta Hot Potatoes.

Experiência adquirida pela análise dos resultados

Este cxante visava focalizat dois pontos principais: o prineiro cra com relaçăo ao conteŕdo do exane enjas alteracoes sofridas formum gutadas pela experiencia anterior proporemonda pelo exame de Abril de 19998. Disponibilizaram-se mais informaçoes que

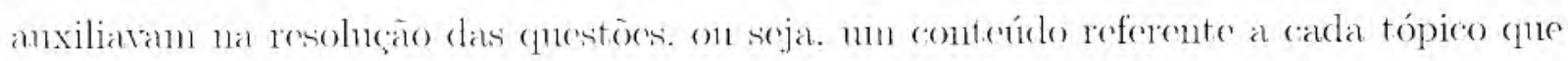
estava sendo avaliado. () segunde ponto principal cra descobrir os tipos de questôns da ferramenta frocware mais propícios para cada tarefa do Exame. Os resiltados em ambos aspectos foram positivos havendo colaboração por parte do grupo que participou do exame piloto através de críticas que anxiliaram a avaliação do conteńdo e a informatização (lo exane. Todos os participantes se manifestaram favoravelmente ao fato do conteído voltalise a textos científicos (mesma visio obtida no EPI aplicado en Abril de 1998). 


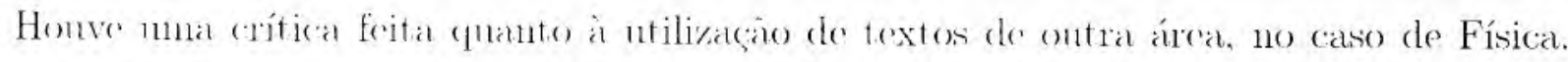
anmentanulo o nived de difienledade do exanne. pois a falta de familiaridade com os assuntos

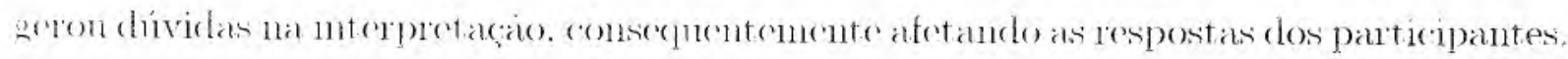

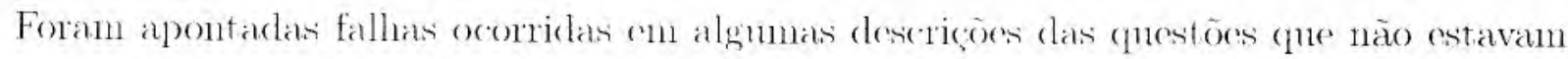

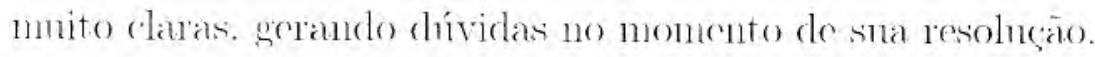

Como o exame foi claborado com o anxílio do computador. pretendia-se investigar se a forma com que o software possibilitava apresentar as questōes era satisfatória on nâo. (Os participantes foram favoráveis ao formato de apresentação proporcionada pelo software. O exame channon a atença para dos pontos principass. O primeiro, relativo ao conterido. demonstrava a necessidade de se especificar mais daranente as questoos. Na realidade. o cedarecinento deveria partir prineiramente dos objetivos pretendidos em

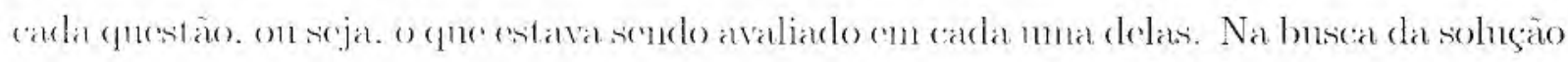

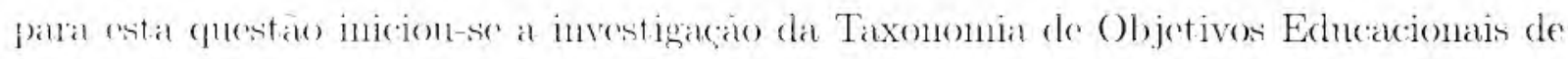
Bloom descrita no Cappítulo 4. () estudo das classese estabelecidas por Bloom (1956)

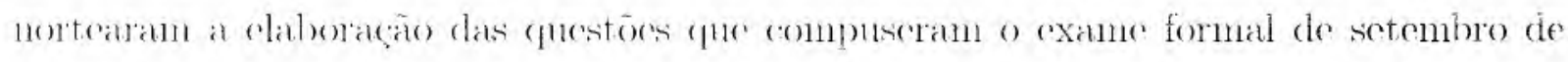
2000. () segmudo ponto. relativo a informatização. referia-se a dois aspectos: à segurança C ao sistema de pontuagaio/nota de corte, pois a pretençäo cra elaborar exames realizados no computiador.

A ferramenta freoware ntilizada na constmęào deste exame năo possibilitava bloguear os acesonos as respostas das questoes visto que ela gerava páginas HTML em que o estudante poderia. através da opģà "Exibir código fonte" do nemen checar as respostas. Apesar de podernos sallar este problemar pois a ferranenta possui código aberto.

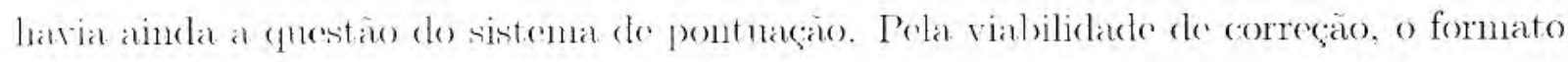

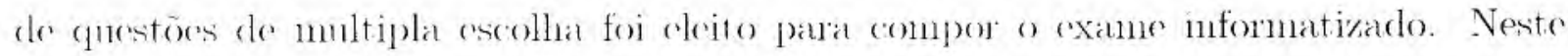

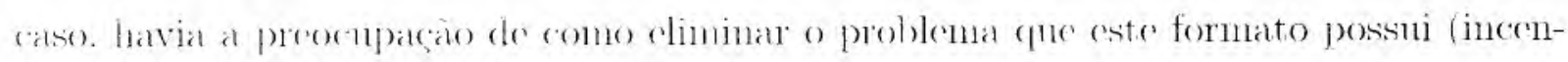

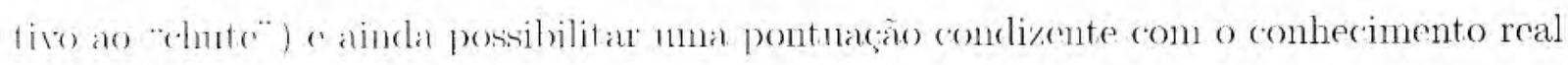
(lo cstudante. 1uäo se restringindo as porentagens de acorto como a ferramenta freeware proporcionava.

Assim. iniciaram-se os esturdos do método Medicla de Probabilidade Admissível descrita no Capítulo 5. Estes dois pontos principais incentivaram a implementação de um 


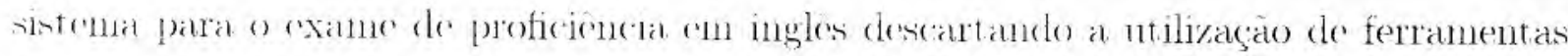

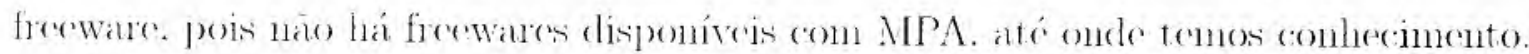

\subsection{Exame formal realizado em Setembro 2000: MPA no formato lápis e papel}

\section{Aspectos Gerais}

() EPI (com esturlantes de mestrado regularmente natriendados no ICMC-USP) realizado em 27 de Setembro de 2000 foi claborado pela Profa. Dra. Sandra Maria Aluísio o aplicarlo pela Profa. Dra. Maria da Graca Pinentel. () exame foi amparado pela Taxonomia de Bloom para a clabolação das questós e pelo método MPA para pontuaçào. For aplicado em papel e corrigide com o anxílio do compntador por meio de planilha

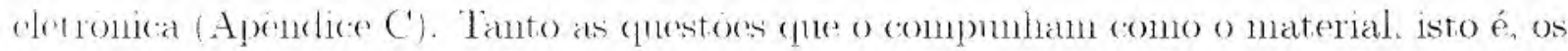
artigos científicos cann ignais para fodos os participantes. Cinco ahunos participaram do exame. sendo que quatro deles foran aprevados.

\section{Experiência adquirida pela análise de resultados}

Anparade pela Taxonomia dr Bloome o conterícto do exame pretendia apresentar-

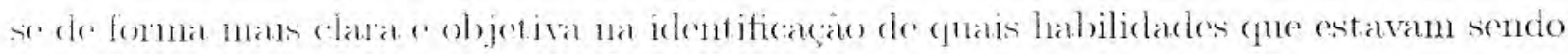
avaliadas. atendendo ans asperetos verificades no exane piloto de Setembro de 1999. Outra pretencaion foi a avaliaciano do mótodo MPA utilizado con o propósito de sanar alguns problemas identificados com relaçäo à pontuacão do exanne. Para verificar o ponto de vista dos estudantes que fizeram o exane formal. for solicitado o preeenchimento de um

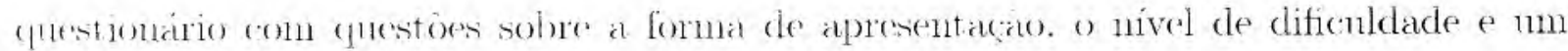
(anupo live para conentános genéricos. Dos 5 participantess 4 manifestaram sta opinião. Honve a aprovaçáe da forma de aperesentação. salientaudo que náo oferecia problemas de interpretacäo (comprovando o anxílio proporeionado pela Taxomomia de Objetivos

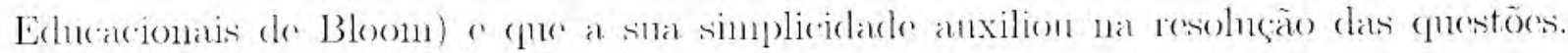

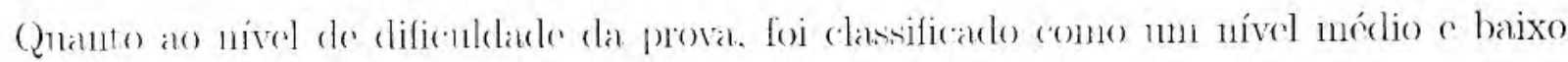

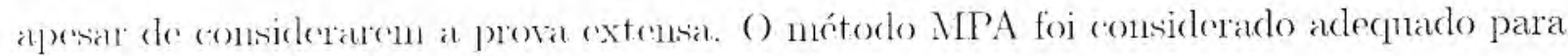

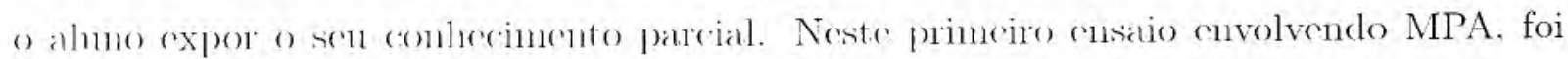




\subsection{Exame formal realizado em Setembro 2000: MPA no formato lápis e papel}

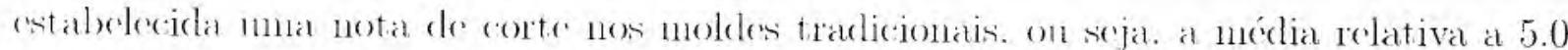

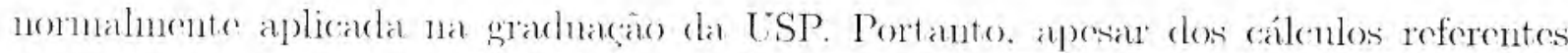

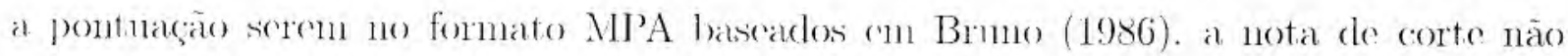

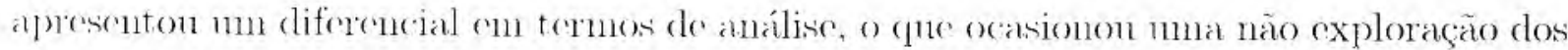
reatis benefícios do método. on seja. a vismalizaciano do conbecinento real dos estudantes. () cálculos da pontuaçà foram estabelecidos con o anxílio de muna planilha eletronica o foram apresentados aos estudantes como mostra a Figura 6.2. Nesta figura vemos que o alıno é aprovado se consegruin uma pontuação ajustada maior on igual a 700 , que é a metade dos pontos conseguidos (nuando todas as (questóns estào corretas (nota máxima).

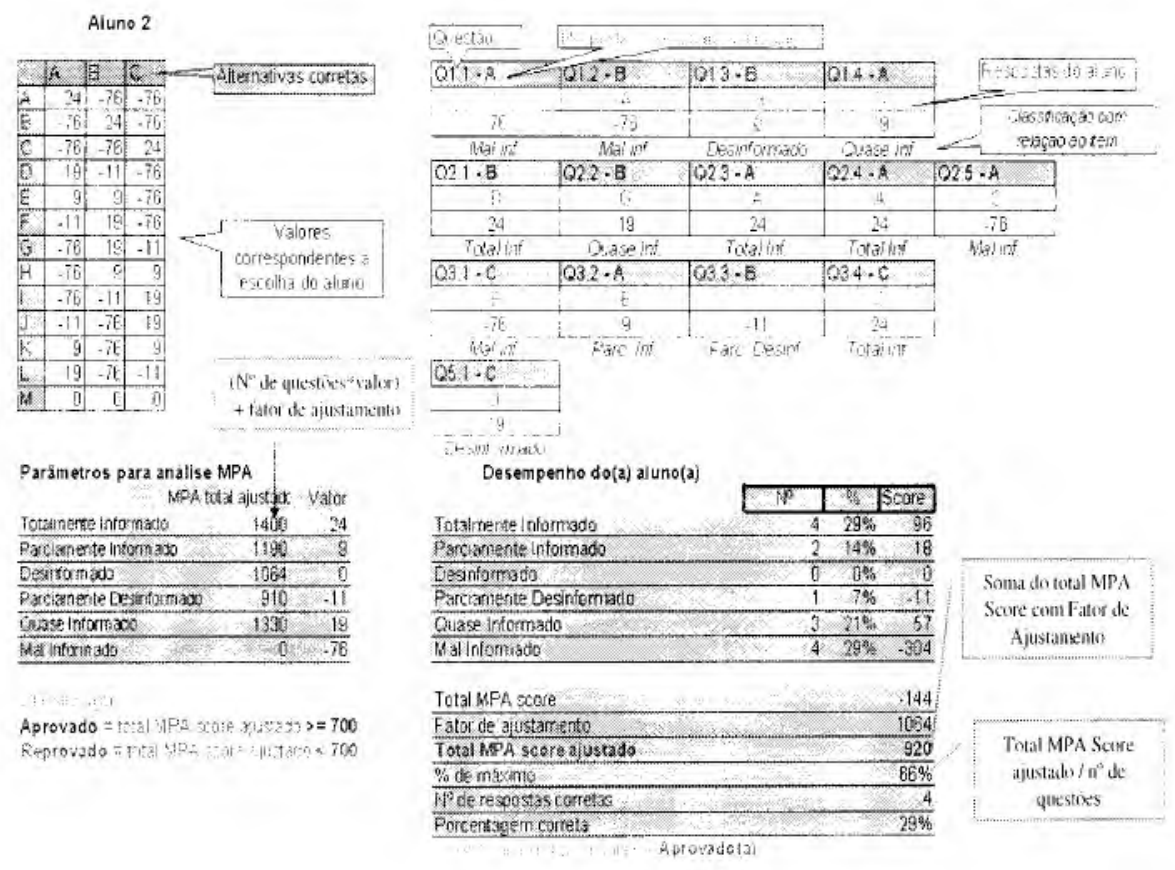

Figura 6.2: Aprosentaçà cios resultados individuais do exame de Setembro de 2000

Pelas tabelas de respostas dos alunos que fizcrame o exame de Setembro de 2000 comprovaram-se os resiltados da experiencia de Shuford \& Brown (1974), em que nos prinkeiros exannes utilizando MPA os alunos năo exploran devidanente os recursos que o método proporciona (ponca ntilizaçao das alternativas intermediárias). Devido a este fator e à forma enu que a nota de corte foi estabelecida. verificon-se que ele aproximon-se de im exame con questóes de múltipla escolla tradicional. A nota de corte deveria ser repensada. Esta modificacăo ocorren no Exame formal de Abril de 2001. 


\subsection{Exame piloto realizado em Fevereiro 2001: Exa- me Online}

\section{Aspectos Gerais}

O exame piloto do Fevereiro de 2001 foi totaluneute informatizado - o estudante

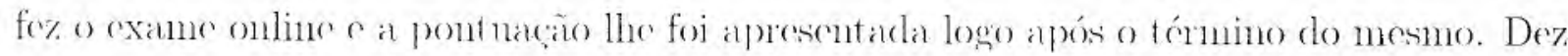
panticipantes. cutre oles mestrandos e dontorandos da área de computação anxiliaram na araliacão do sistema piloto claborado para o exance. Este exame ntilizon as mesmas questors do EPI realizado en 27 de Setembro de 2000 com o acrescimo de mais uma parte com 7 enestors. totalizando 21 gnestions. " no final do exame o ahno recebia muna tela

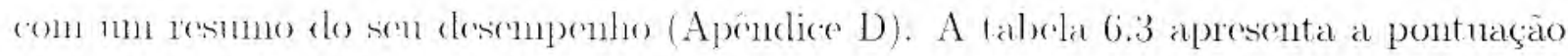

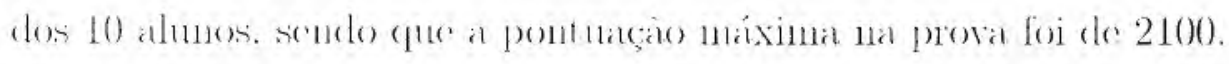

\begin{tabular}{|c|c|c|c|c|c|c|c|}
\hline Alumes & $\begin{array}{l}\text { Pont nacios } \\
\text { Total }\end{array}$ & $\begin{array}{l}\text { Totalmente } \\
\text { Informade }\end{array}$ & $\begin{array}{l}\text { Parcialmente } \\
\text { Informato }\end{array}$ & $\begin{array}{c}\text { Quitise } \\
\text { Iuformado }\end{array}$ & Desinformido & $\begin{array}{l}\text { Tot:alncute } \\
\text { Mul Informado }\end{array}$ & $\begin{array}{l}\text { Parcialueute } \\
\text { Mal Informado }\end{array}$ \\
\hline $\mathrm{Al}$ & $19, \bar{s}$ & 17 & 1 & $\mathrm{i}$ & 1 & i & 0 \\
\hline 12 & 1755 & 15 & 0 & 2 & 0 & 7 & 1 \\
\hline $\mathrm{in}$ & $15: 19$ & $1 i i$ & 3 & $\mathrm{i}$ & 6 & 3 & 11 \\
\hline 14 & 1600 & 16 & 18 & 13 & 4 & 5 & 0 \\
\hline Ai & 1511 & $s$ & 3 & 1 & 1 & $\overline{5}$ & 0 \\
\hline $\mathrm{M} 6$ & 1470 & 13 & 2 & 0 & (1) & 6 & 0 \\
\hline$A^{7}$ & IXS: & 11 & 0 & 3 & 0 & 7 & 0 \\
\hline$A x$ & $1: 5 \%$ it & 12 & 2 & 0 & 0 & 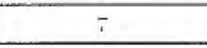 & 0 \\
\hline $1+1$ & $123: 31$ & $6 i$ & 3 & 3 & 0 & 7 & 4 \\
\hline int & 1111: & s & $!$ & 2 & it & $\Rightarrow$ & 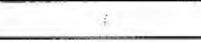 \\
\hline
\end{tabular}

Tabelar 6.3: Classificacáo dos móchulos com base na Taxonomia de Bloom

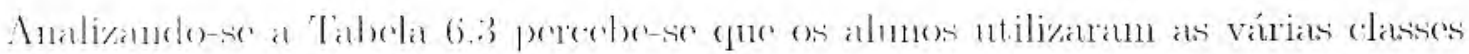

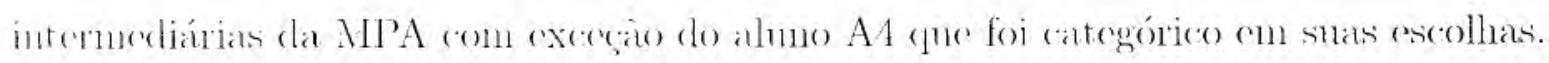

\section{Experiência adquirida pela análise dos resultados}

Por so tratar da mundanca do apresentaço para 1 mu formato informatizado, uma

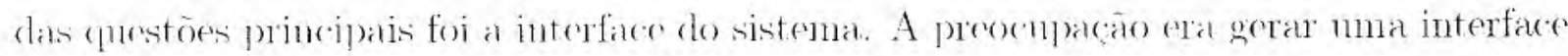
gue nay interferisse no desempenles do estudante. Assim. para o projeto da interface. procuron-se. na medida do possível. fornecer dados ao alumo que sào conseguidos no estilo p)esemetal. por exemplo. as instruçoes o explicaçoes para cada parte do exame, mesmo estande conscientes de (uue näo há possibilidade, pelo menes até o presente momento, de ignalar os deis formates (presencial o informatizarlo). Foi nat tentativa de deixar todas 


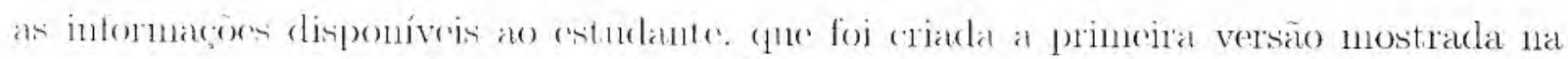

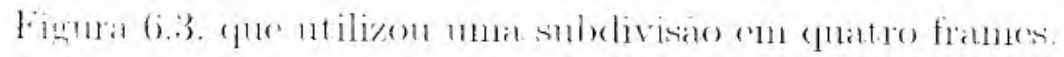

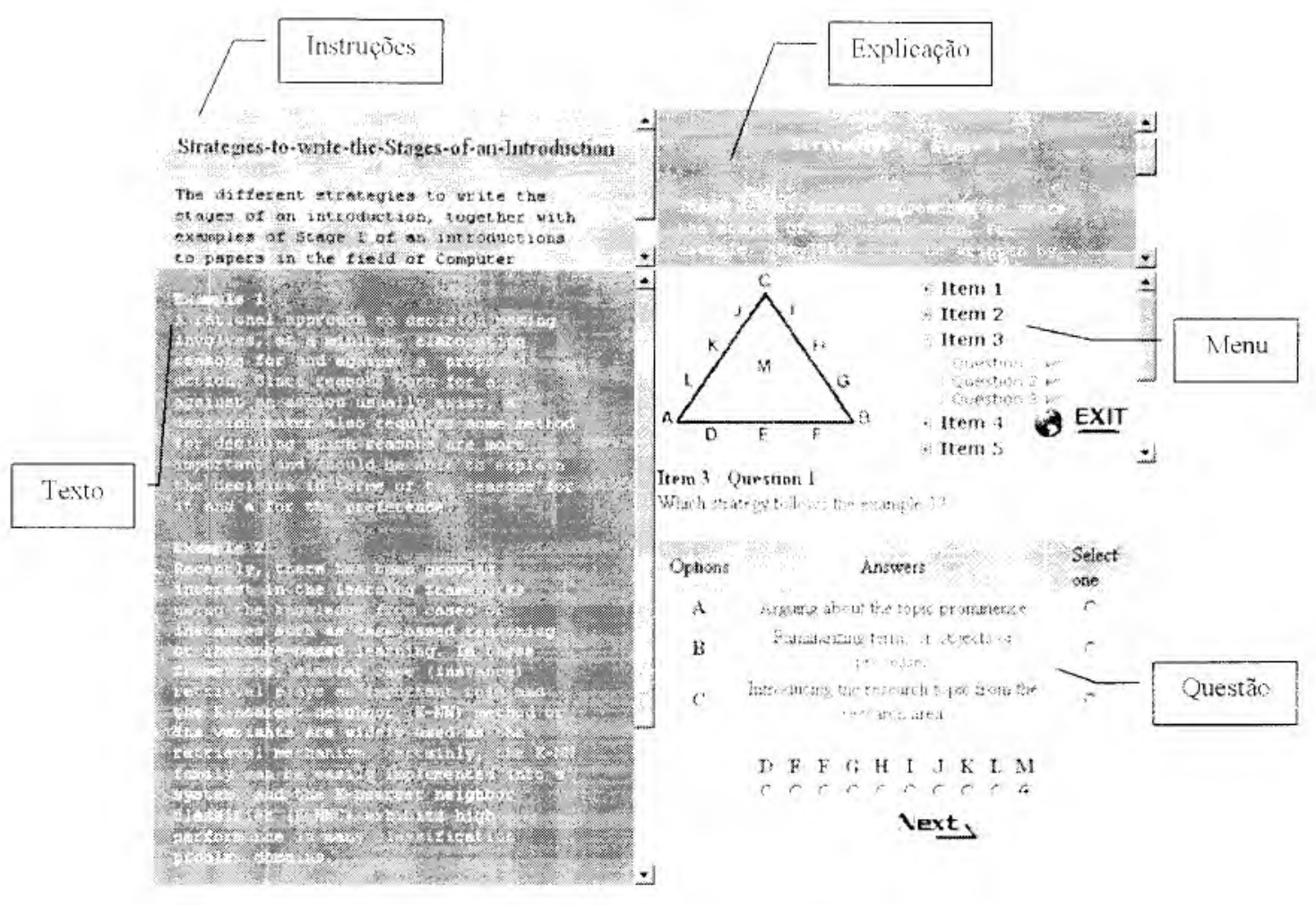

Figura 6.3: Primeilat Interface do Sistemua

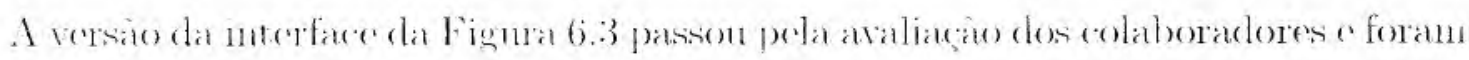

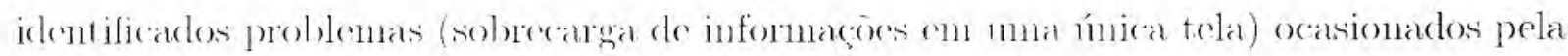

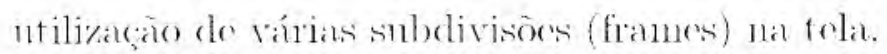

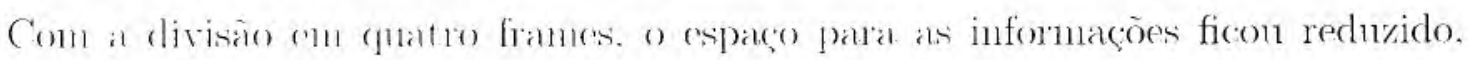
ocasionande a uecessiclade de rolagenu de tela. Os colaboradores alegaram que isto prejudicava a leitura pois näo consegulann chegar diretanente à informaçà desejada. Alegaram

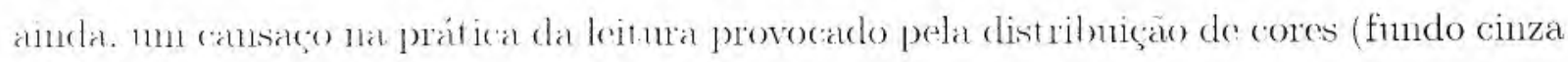

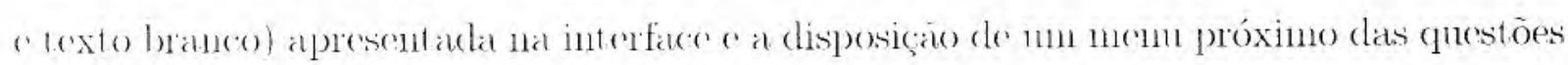
confundia os colaboraclores. Nos aspecetos referentes ao conteríclo do exame houve muna

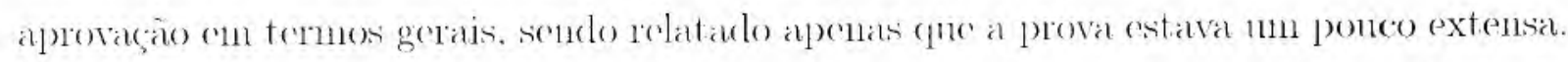

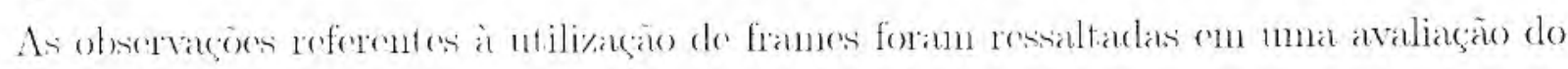

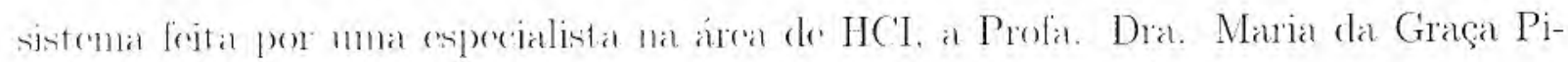




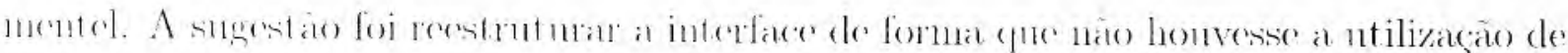

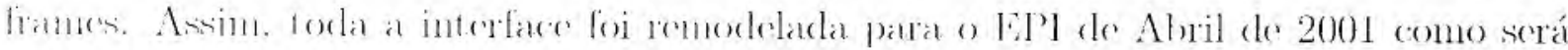
mostraclo na póxima seçio.

\subsection{Exame formal realizado em Abril 2001: MPA no Exame oficial online}

\section{Aspectos Gerais}

() EPI realizado enu 11 de Abril de 2001 (com estudantes de mestrado regulaumente

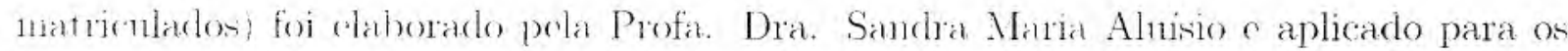
alunos das áreas do Computacăo. Natemática Computarional e Estatística. 1utilizando o

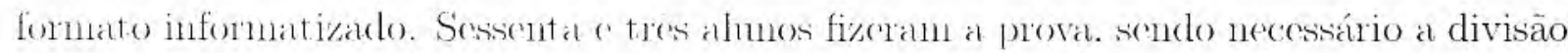

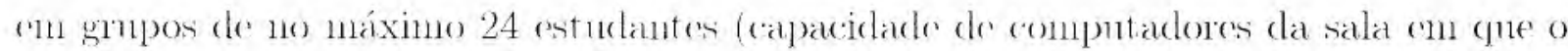

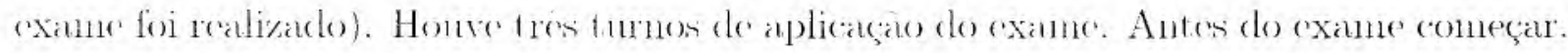

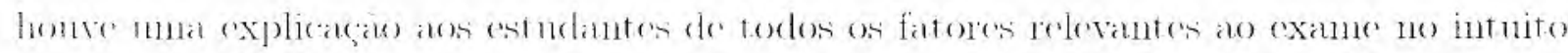

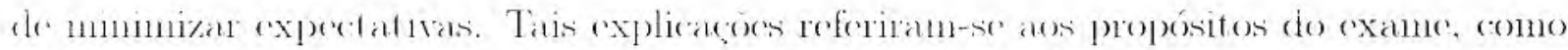

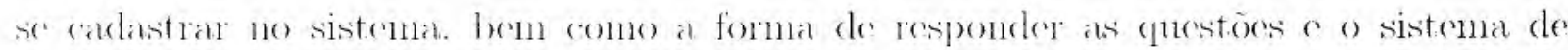

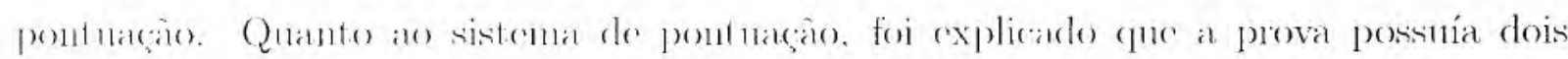
grander módulos com duats notas de conte diferentes. () Mónhulo 1 (Estrutura de textos

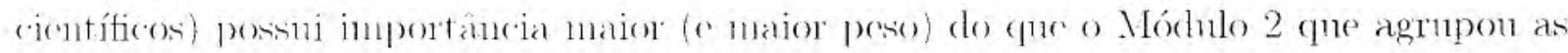

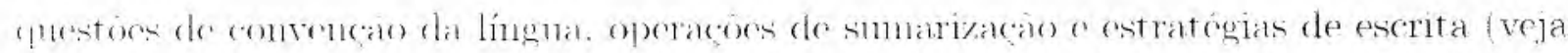
Figna 6.8$)$

\section{Experiência adquirida pela análise dos resultados}

Com hase nas experiencias alcancadas no exane piloto de Fevereiro de 2001 foi

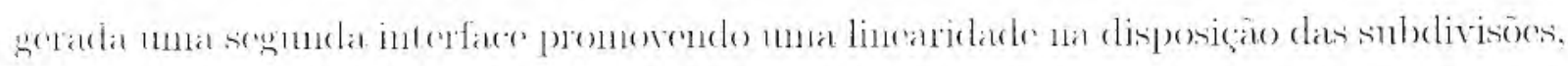

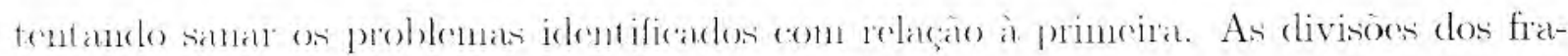

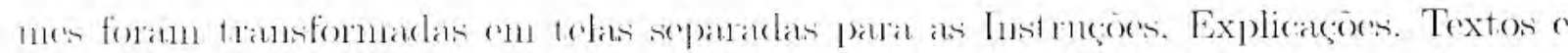

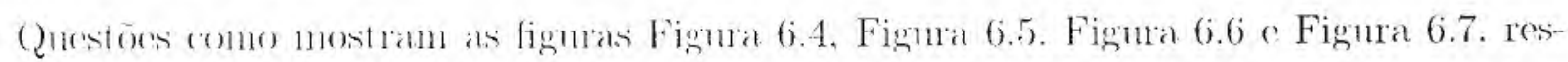
pertivinneite. 


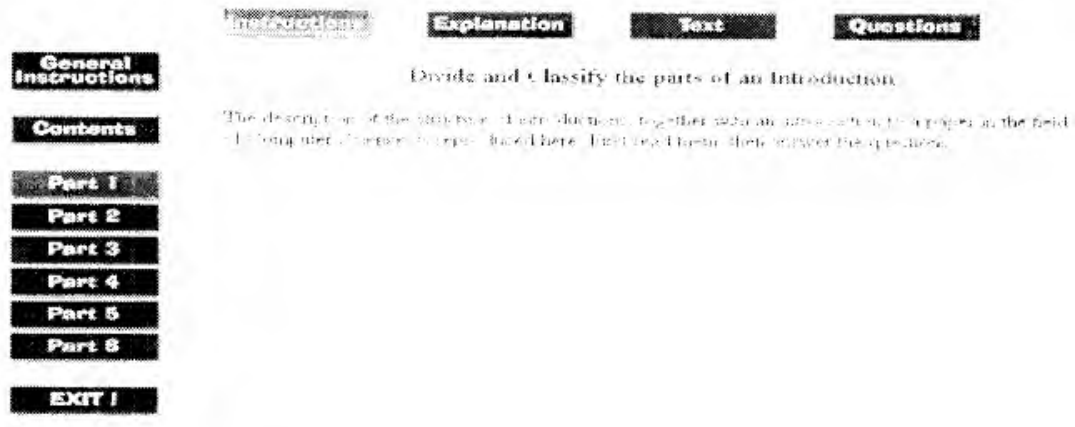

$\angle$ Bock

vexts

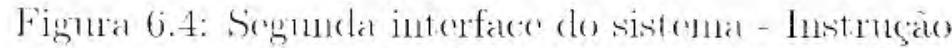

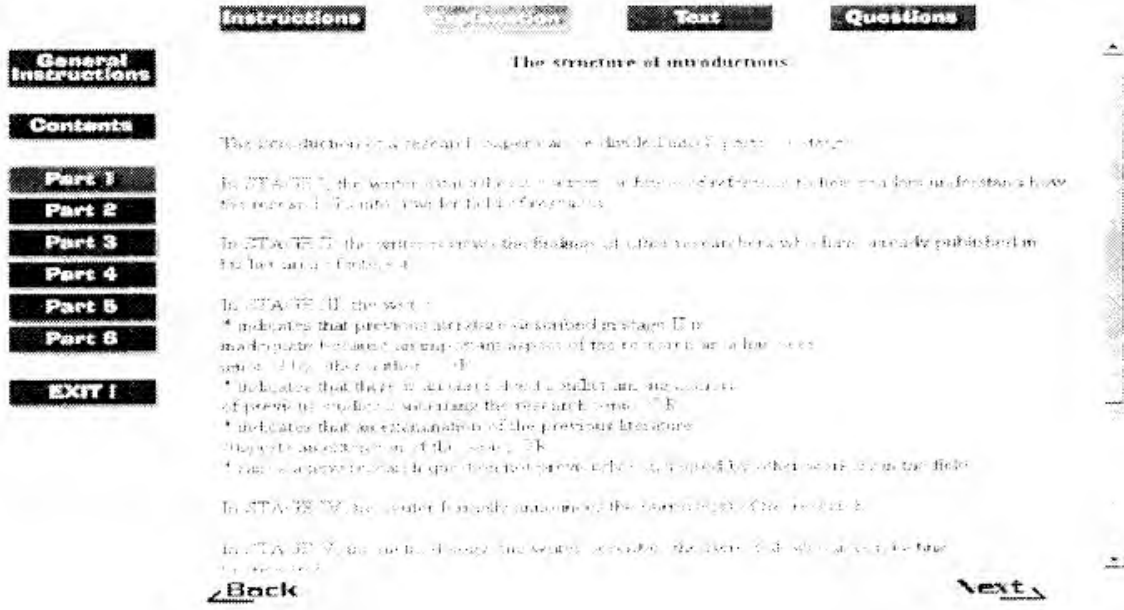

Figura 6.5: Segunda interface do sistrma - Explicaçōo 


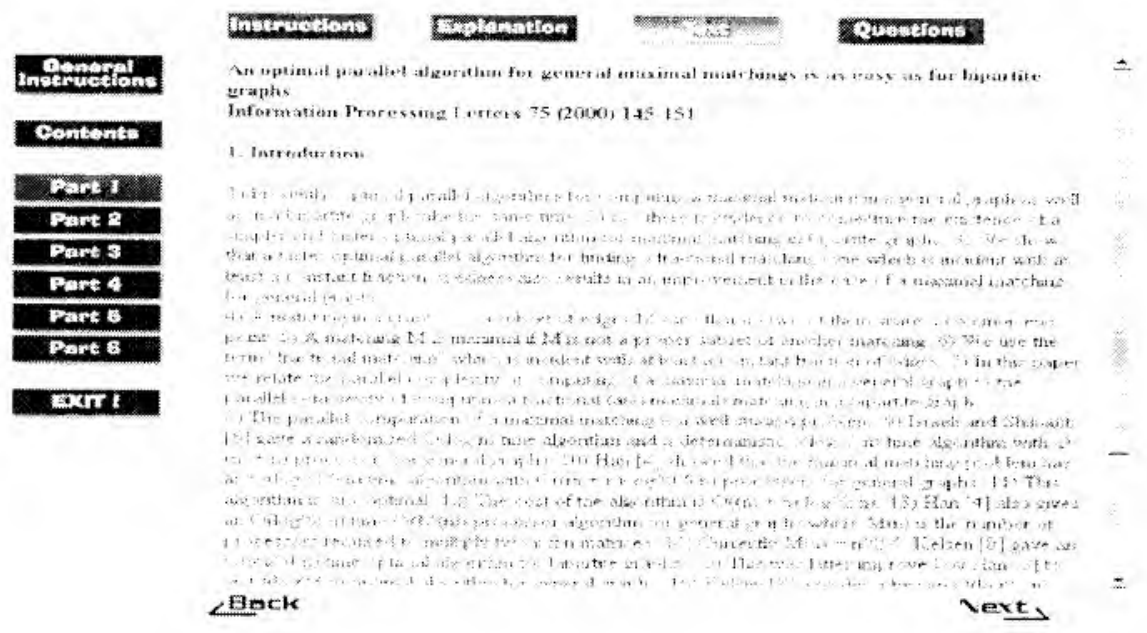

Fignar 6.6: Srgunda interface do sistruna - Texto

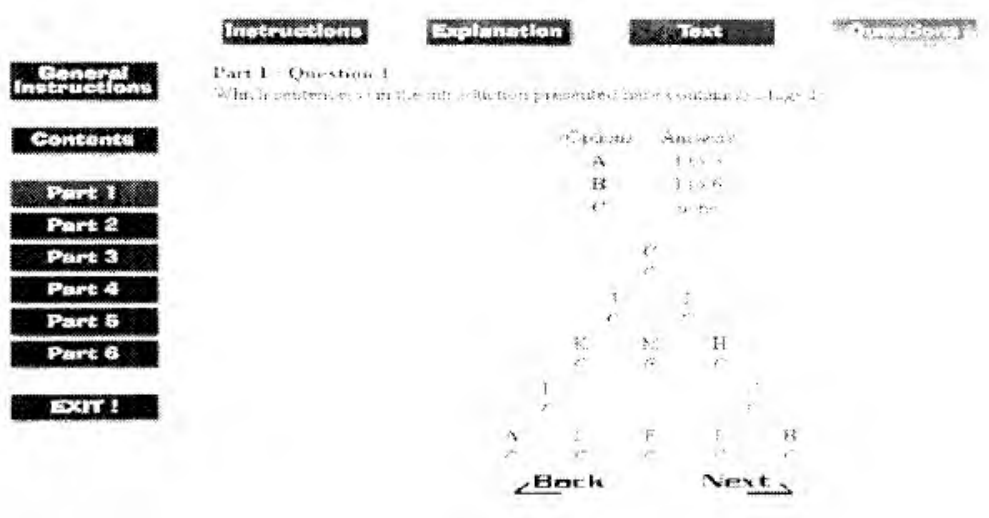

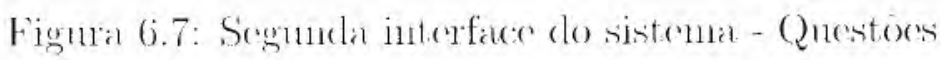




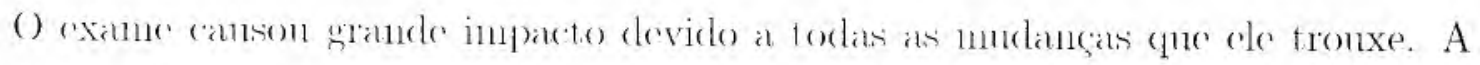

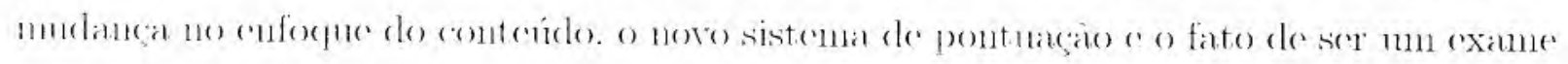

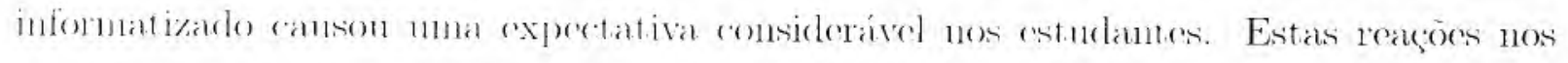

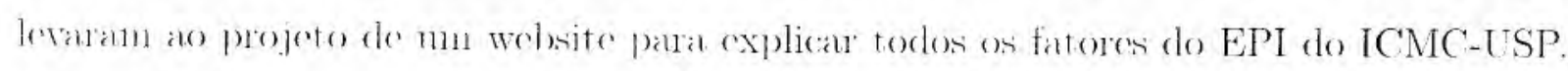
()s pontos problemáticos identificades neste exane foran:

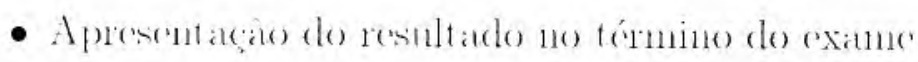

Após o estuclante finalizar o exanne. nnla tela referente a sua pontuação juntannente

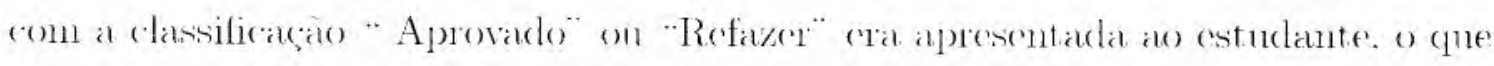
(x):

- Interpretacian deresultados

Foj necessário nuna explicacaio individual a respeito da tola apresentada. pois o sistema nado formecia uma tela explicativa do conno deveria ser intepretada a tabela de desempene disponibilizada no final do exane (Fignua 6.8):

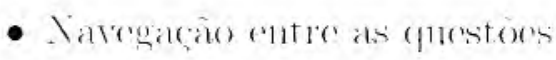

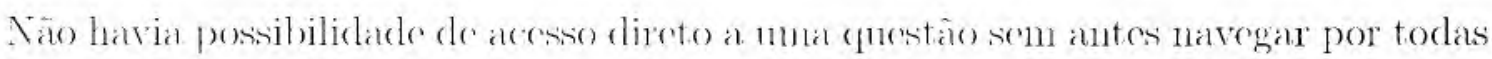

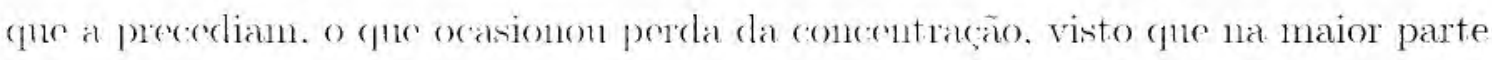
dos casos o estudante saja da tela das questoes porcune necessitava relembrar algo

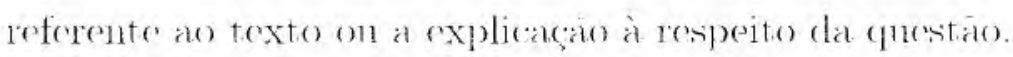

- Leitiura do textos

A leitura do textos lomgos na tela do computador foi considerada difícil pela grande matioria des esturdantes:

- Dificuldacles nistó (puestom

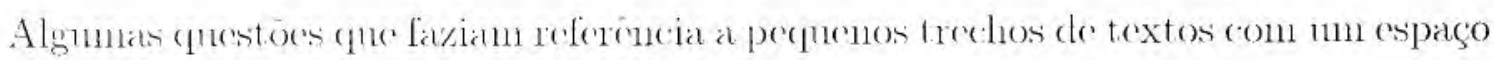
para sere completade se tormatran difícois de resolver por näo estarem acompanhadas

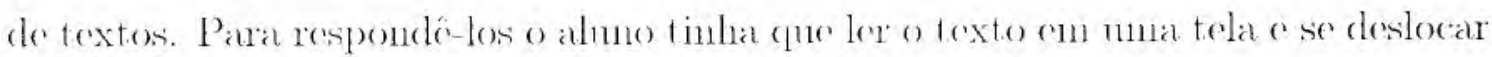

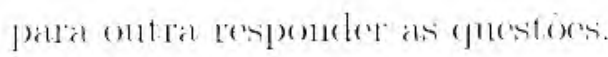




$\begin{array}{lcc}\begin{array}{l}\text { Desempenho do(a) } \\ \text { Conhecimento }\end{array} & N^{\circ} \text { de Questóes Score Parcial } \\ \text { Totalmente Informado } & 0 & 0 \\ \text { Quase Informado } & 14 & 266 \\ \text { Parcialmente Informado } & 0 & 0 \\ \text { Desinformado } & 0 & 0 \\ \text { Parcialmente Desinformado } & 4 & -44 \\ \text { Mal informado } & 2 & -152\end{array}$

Score do Módulo 1: 635

Score do Módulo 2955

Classific açăo: REFAZER!

\section{Dados da Prova:}

$\begin{array}{lr}\text { Score Maxmo } & 2000 \\ \text { Numero de Questóes: } & 20 \\ \text { Nota de Corte do Módulo 1: } & 704 \\ \text { Nota de Corte do Módulo } 2 & 1056\end{array}$

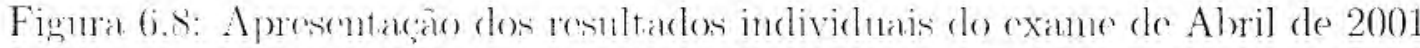

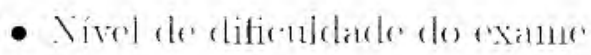

() EPl tratat de ingles instrumental para leitura de textos científicos. Muitos alnnos que fizeran cousos de ingles fradicional tiveran dificuldarle. pois os tipos de

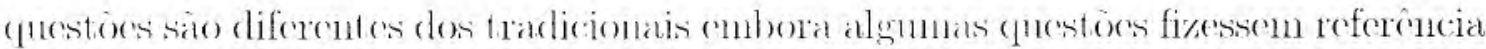

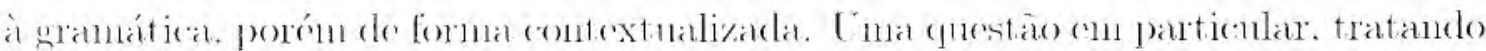

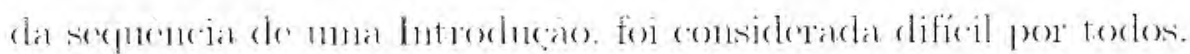

- Notar de corte

A nota de corte dada a experiencia do exane de Setembro de 2000. foi alterada para $11 n$ valor narior ene a média tomando como batse os trabalhos de Bromo (1986),

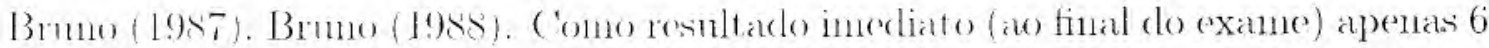

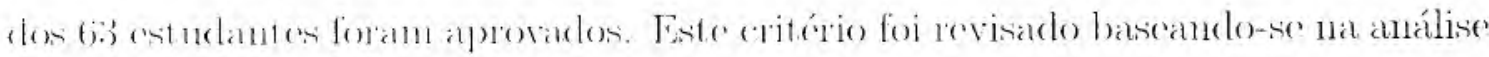




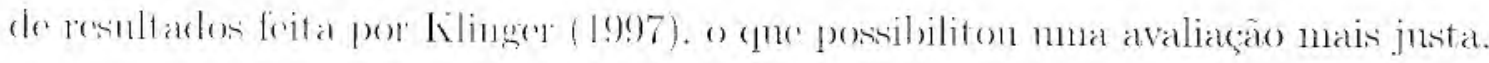

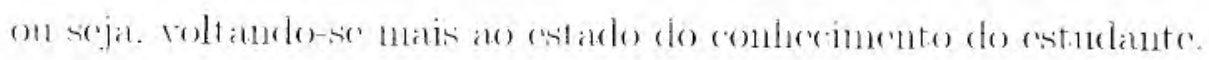

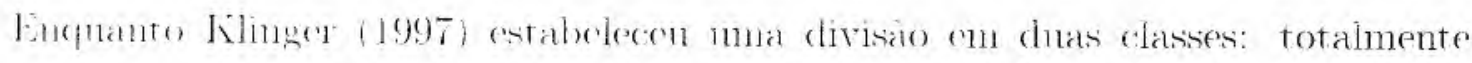
informado on quaser informados a mal informados on desinformados para classificação dos

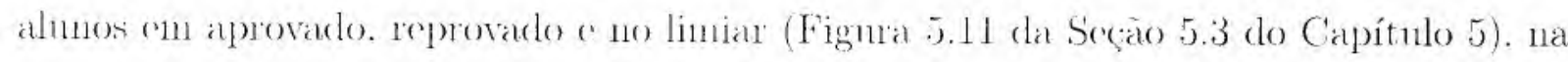

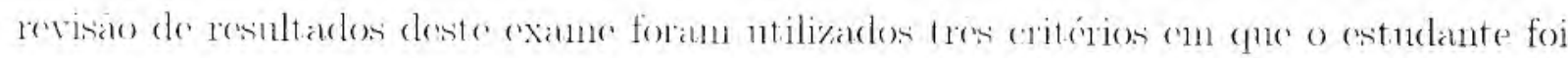

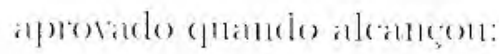

1. $50 \%$ (on nuaisi de respostas na classic "Totalnente Infomado" a $25 \%$ (on menos) de lespostan na (lasise "Mal Informado".

2. 90\% (on mais) de respostas nas dasses "Totahnente Informado". "Qulase Informa-

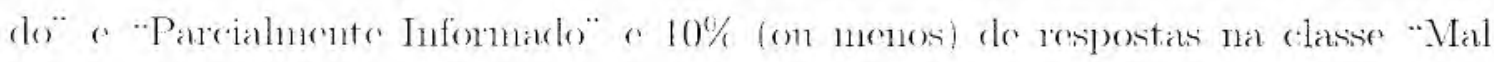
Informate."

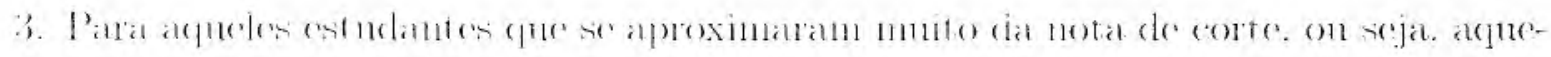

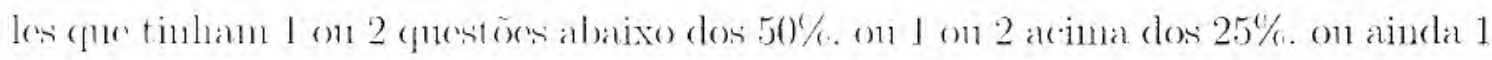

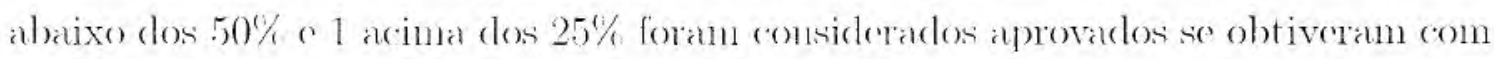

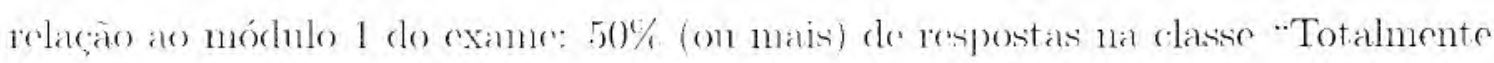
Informado". 25\% (on menos) de respostas nas dasses "Mal Informado". "Desinfor-

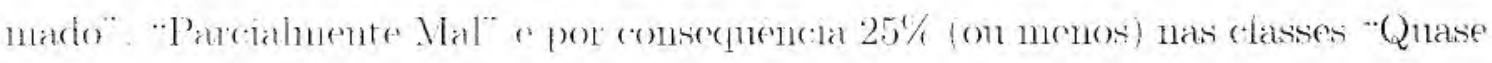
Informado" "Parcialnenter Informarlo".

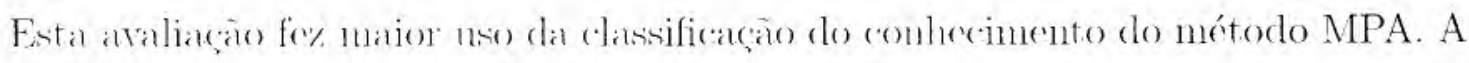

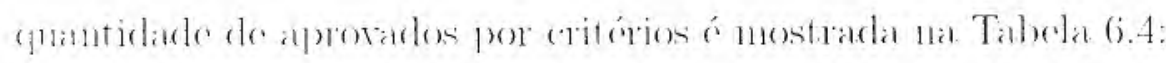

\begin{tabular}{|c|c|}
\hline Critérios & Quanticlarte ale Aprovartos \\
\hline Prinucira & $1: 3$ \\
\hline Segmulata & 1 \\
\hline Tescrira & 11 \\
\hline Total & 25 \\
\hline
\end{tabular}

Tabrela 6. 4: Análise de Resultados do EPI de Abril de 2001 


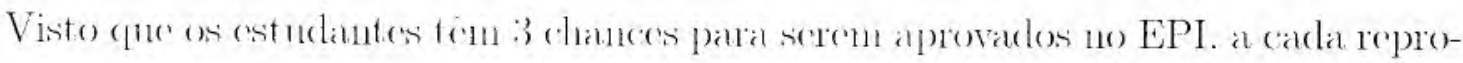

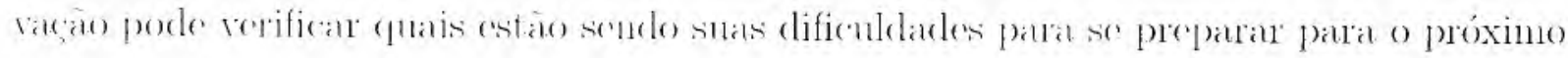

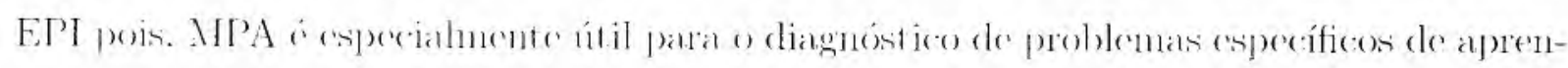

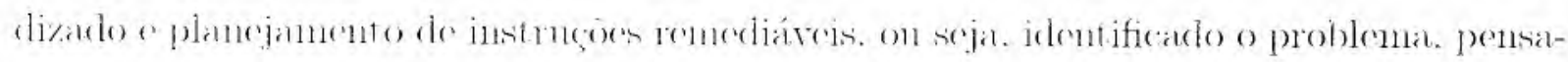

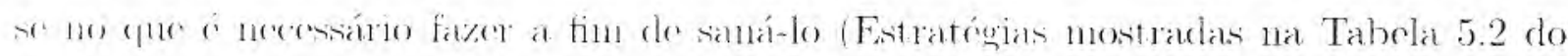
(a)pítulo, 5).

Neste exame formal. toda a teoria relativa ao contenido (Inglês Instrumental). sis-

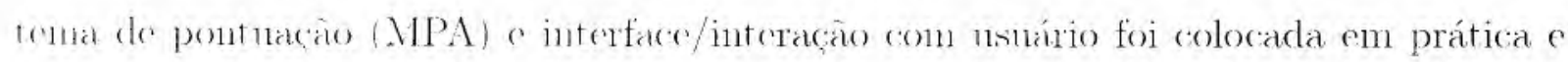

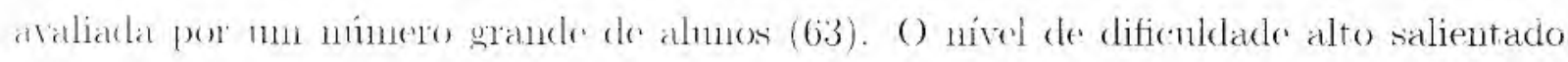

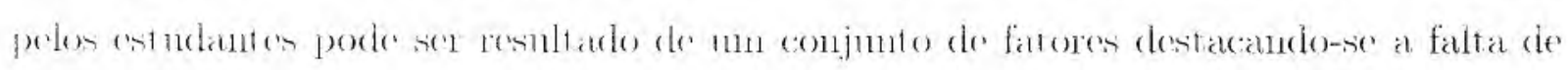

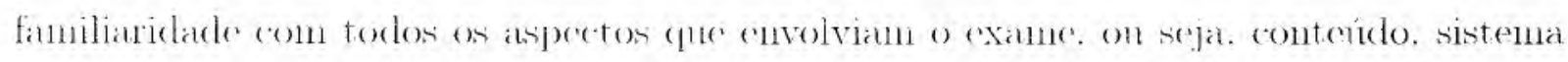

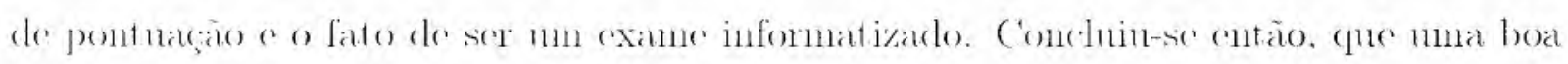

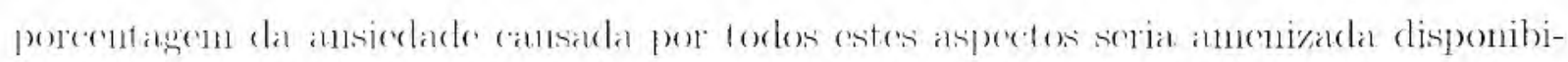

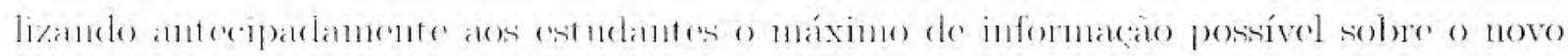

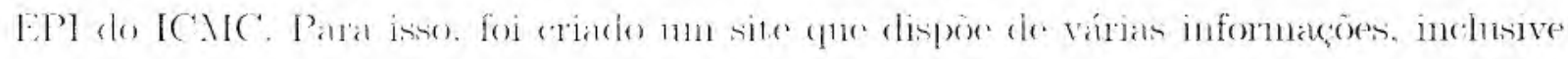

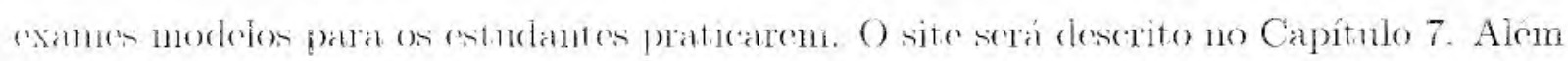
da criacaro do site. honve nma avaliaço da nsabiliclade da interface utilizando a técnica

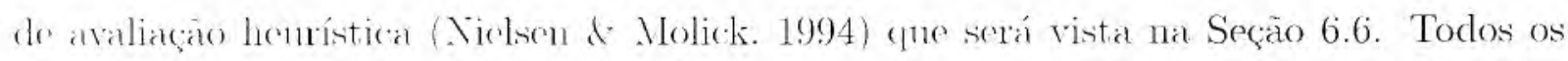

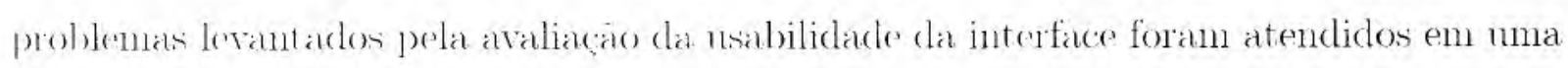
nova versato do EPI a ser ntilizada no Exame de 29 do Setrmbloro de 2001 . For feita ainda

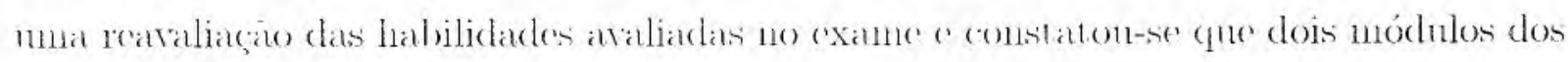

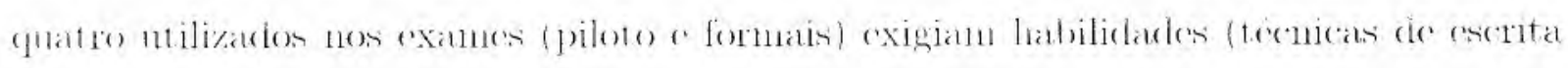

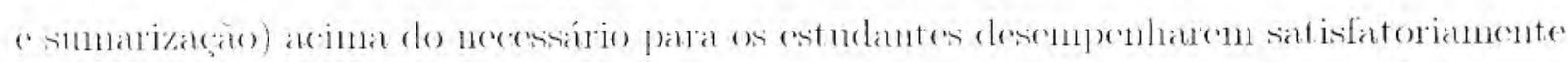

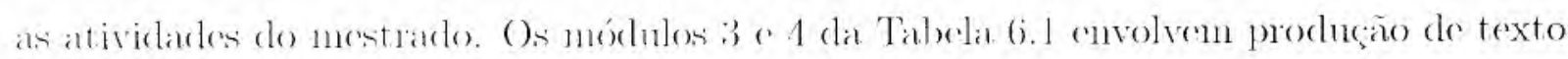

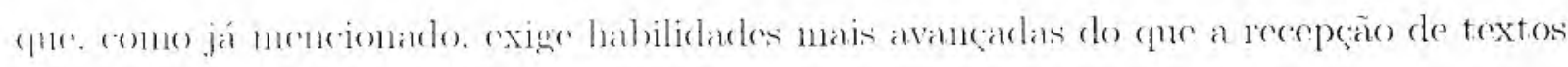

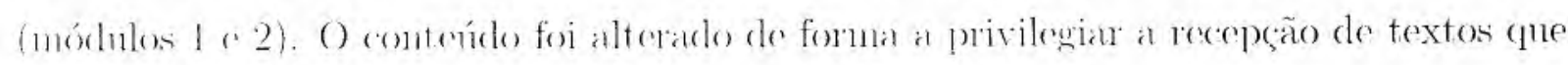

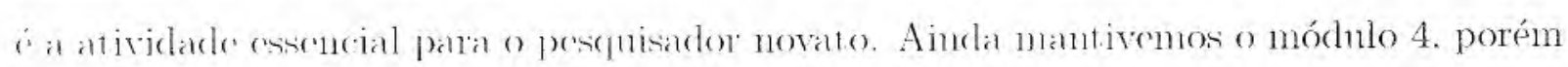

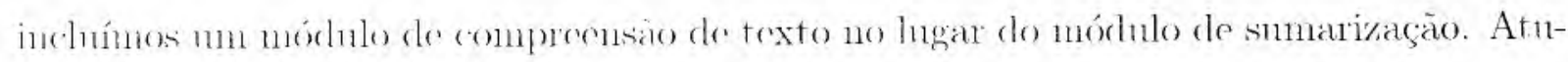
ahnente. os móchulos apresentann a divisáo apresentada na Tabela 6.5. () Apêndice E traz

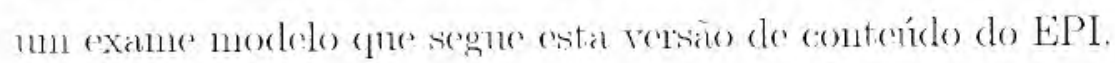


Módulo 1: Convenções da Língua Inglesa para Textos Científicos

(Questóses de múltipla escolla abordando morfologia. vocalunlário. sintaxe, tempos verbais.

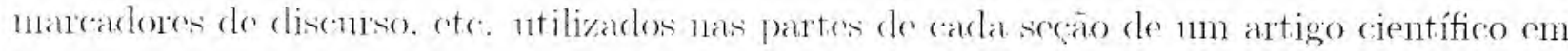
inglen.

Modulo Estrutura de Textos Científicos

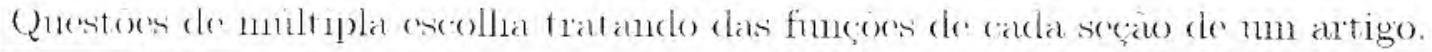

\section{Modulo 3: Compreensão de Texto}

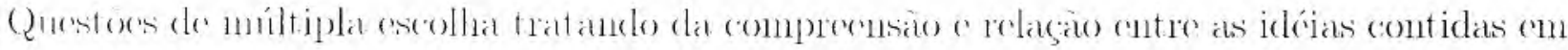
mina secaio de 7111 artigo.

\section{Módule 4: Estratégias de Escrita}

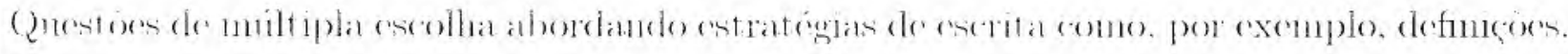

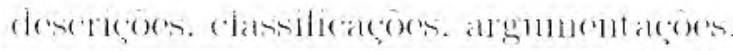

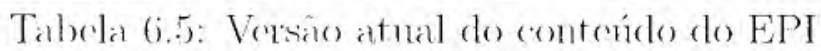

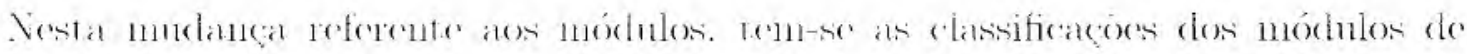

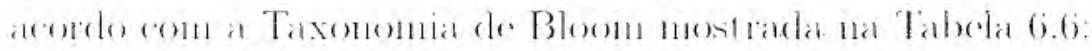

\begin{tabular}{|c|c|}
\hline Módulos & Classe da Taxonomia \\
\hline M1 Convençoes ala Lingula Inglesal paua Textos Cientificos & Conluecimento \\
\hline M2 Estrutula de Textos Cientificos & Análise \\
\hline M3 Comprernsão de Texto & Compreensão \\
\hline M4 Estratégias de Escrita & Compreensão \\
\hline
\end{tabular}

Tabela 6.6: C'ansificacion des módulos eom hase na Taxomomia de Bloom

() segunde aspecto influente no nível das habiliclandes exigiclas foi o material de apoio em inglôs para cuteudimento do tópico (pue se estava avaliando. Como este material

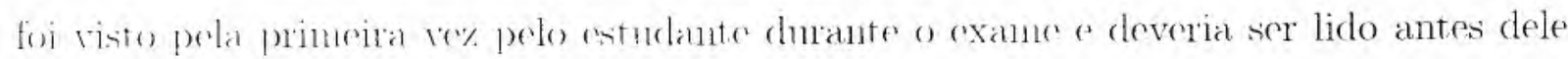

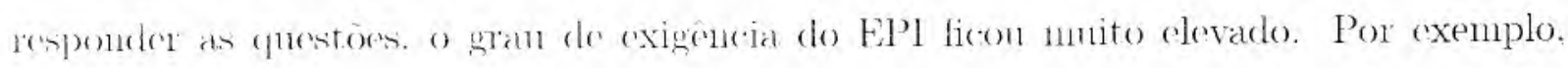
110 módulo 2 o estudante tinha que entender um modelo de estrutura antes de aplicá-lo. Resolvemos avaliar apenats mana habilidade por ve\% a assim remodelannos as instruçoes que 


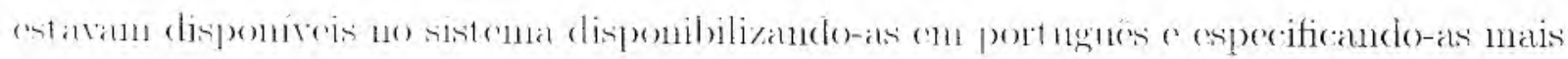

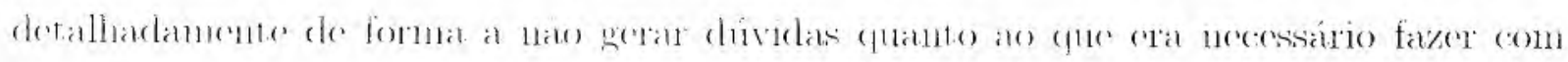

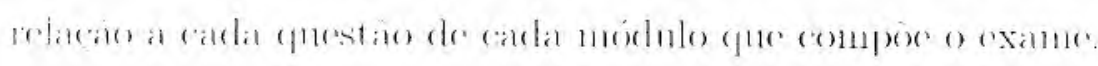

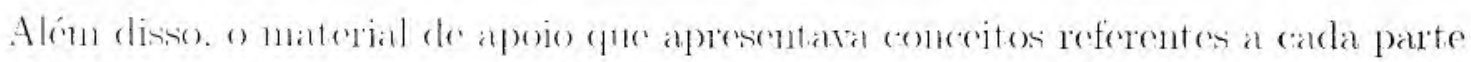

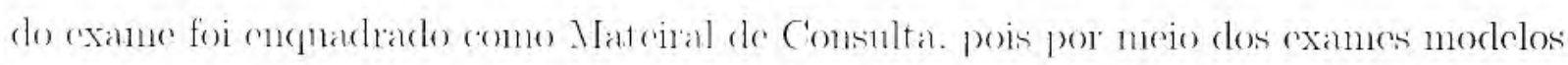
que o site disponibiliza. o estudanto pode verifiér-bo anten do exame fomal. consultando-o

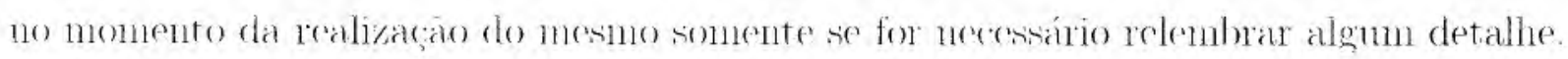

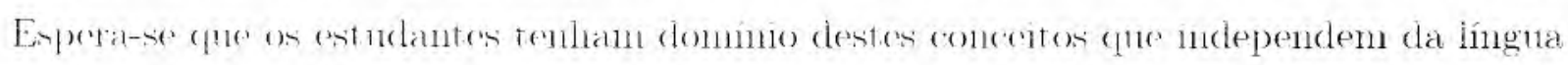

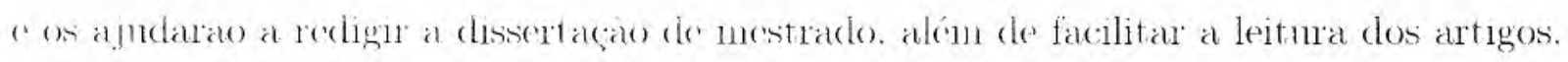

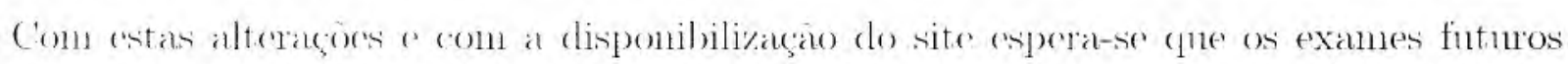
gerem menos ansiedarle por parte dos estudantes semeto o sen desempenho reflexo somente

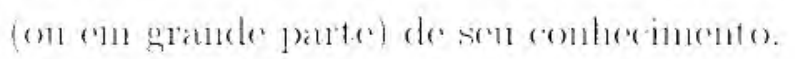

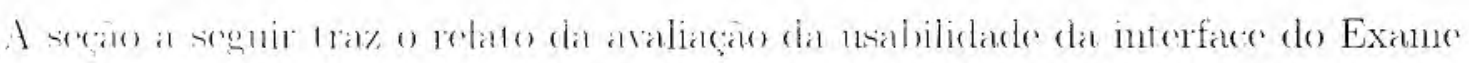

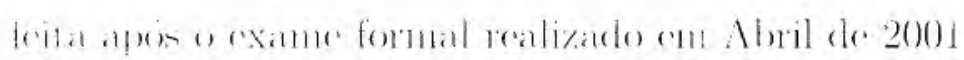

\subsection{Avaliação da Usabilidade do Exame realizado em Abril de 2001}

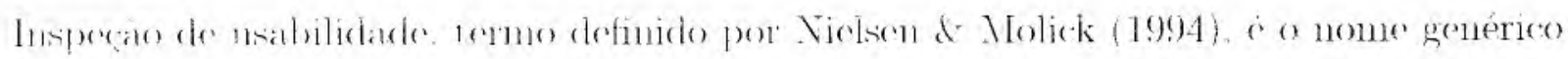

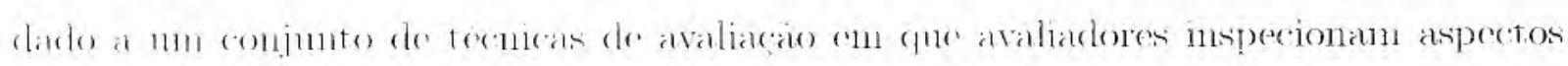
relacionados a mabiliclade do interfaces humano-(omputador.

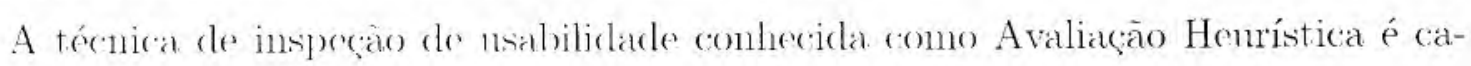

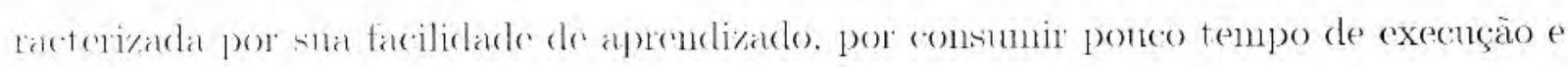

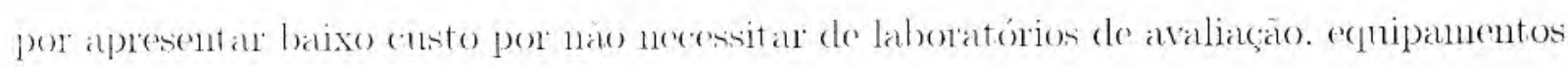

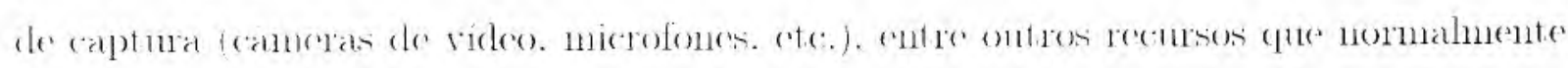

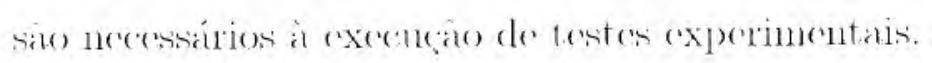

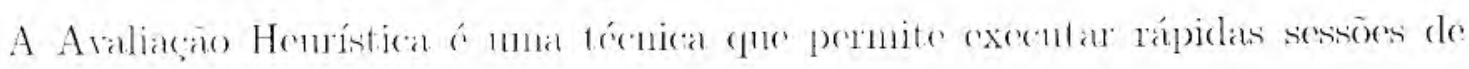

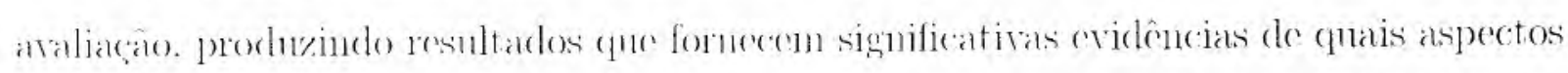

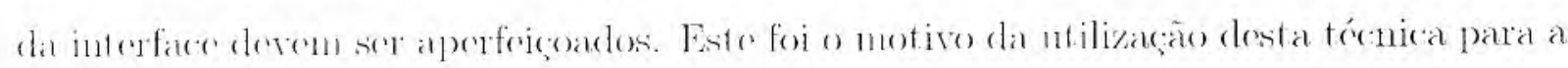

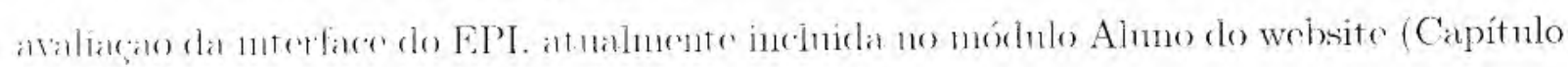


7

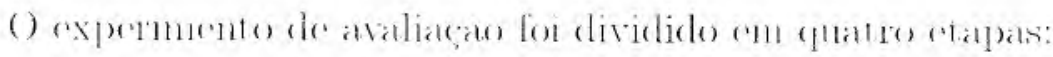

- Planejamento: definiça dos objetivos da avaliaço. seleçao dos avaliadores e definiçao do anmário final do wolsits. CAPTEAP:

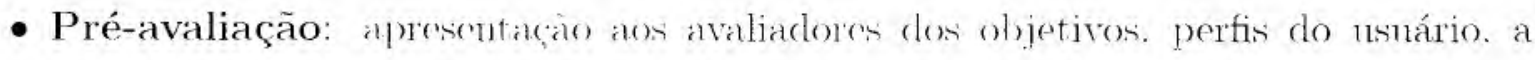

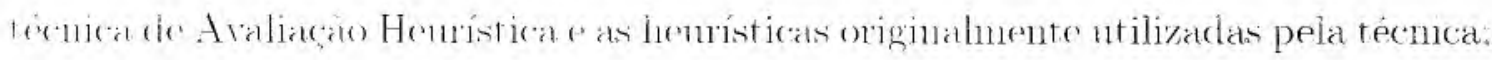

- Avaliação: ralizaçăo de olsectacoes individuais no wobsite. CAPTEAP especifi(anuente no Módulo do Alnuo:

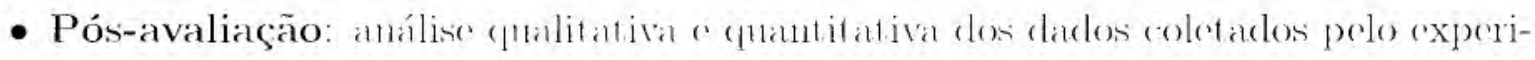

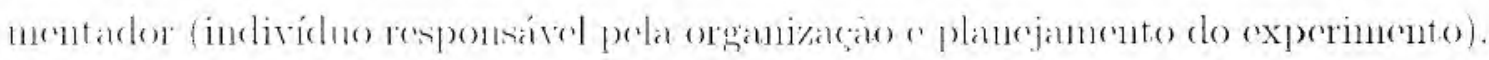

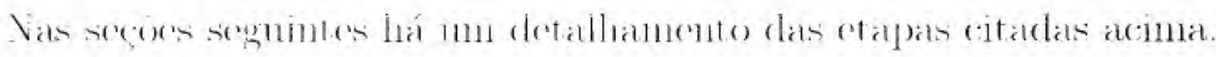

\subsubsection{Planejamento}

\section{Objetivos da Avaliação}

() sistema ('APTEAP inulementa nun exame informbatizado de proficienciar em ingles.

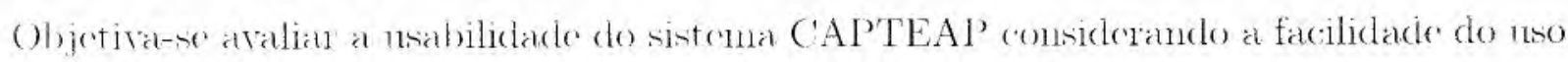
(reficiencia do sistema para se eritar que o sistema interfira no descmpenho do aluno.

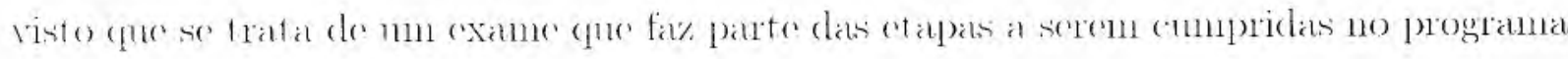

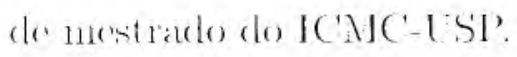

\section{Seleção dos Avaliadores}

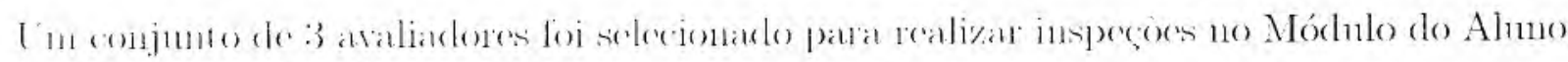
do sistena CAPTEAP. Tokles analiadores pertencian à área de Ciêneia da Computaça

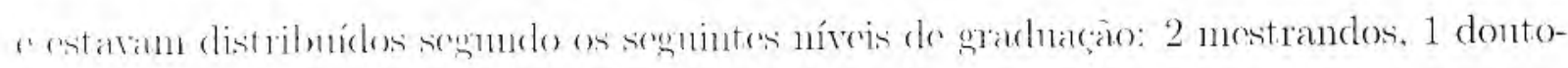
ramele. 


\section{Definição do usuário final do sistema CAPTEAP}

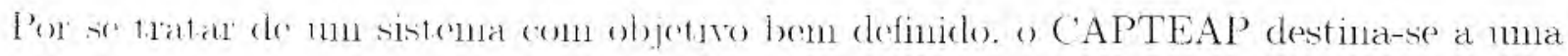

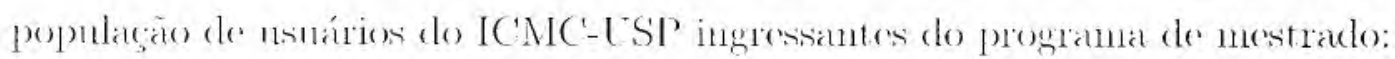

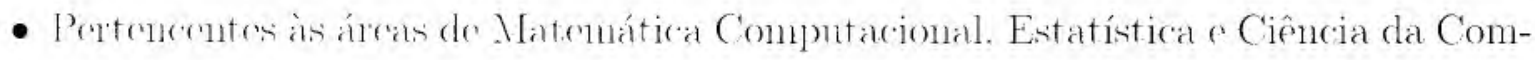
putacas:

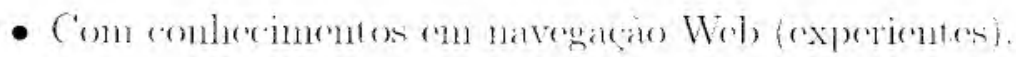

\subsubsection{Pré-avaliação}

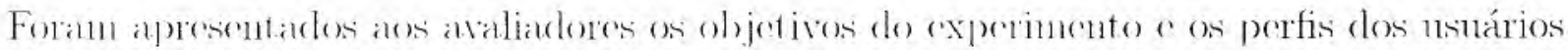

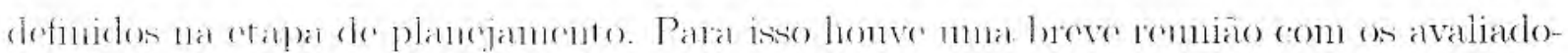
ris.

\subsubsection{Avaliação}

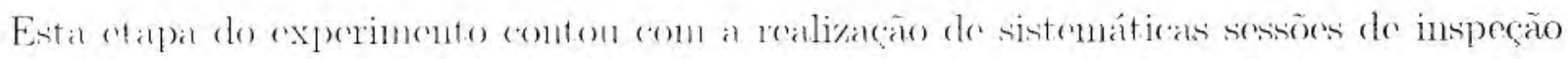

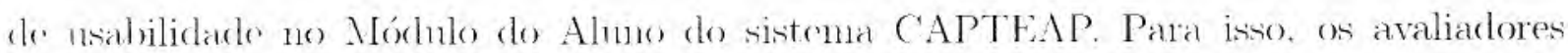

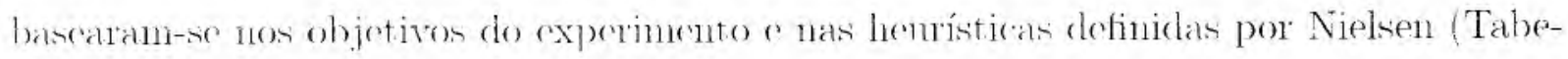
lat 6.7

\begin{tabular}{|c|c|}
\hline \multicolumn{2}{|r|}{ Heuristicas de Usabilidade } \\
\hline $\begin{array}{l}\text { Visibilidade do } \\
\text { Status do Siste- } \\
\text { ma }\end{array}$ & 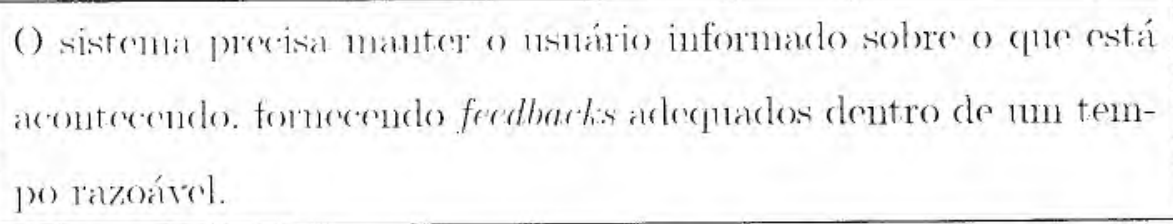 \\
\hline $\begin{array}{l}\text { Compatibilidade } \\
\text { do Sistema com } \\
\text { o Mundo Real }\end{array}$ & 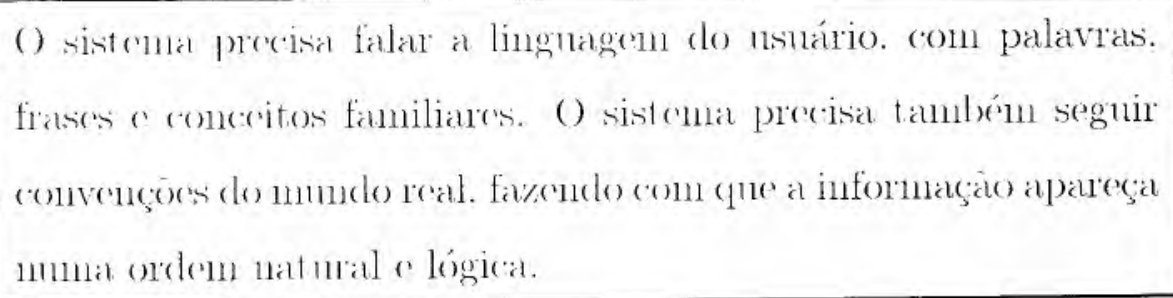 \\
\hline
\end{tabular}

(t)itimal lat próxima página 
(ontinna(a) da páginal anterior

\begin{tabular}{|c|c|}
\hline & Heuristicas de Usabilidade \\
\hline $\begin{array}{l}\text { Controle do } \\
\text { Usuário e Li- } \\
\text { berdade }\end{array}$ & 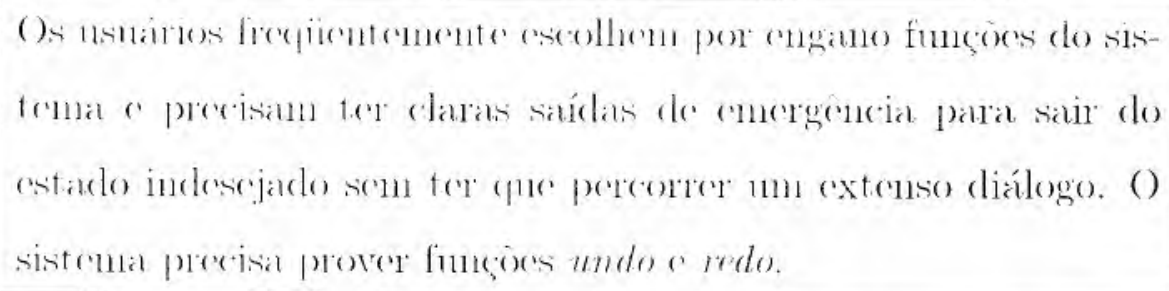 \\
\hline $\begin{array}{l}\text { Consistência e } \\
\text { Padrōes }\end{array}$ & 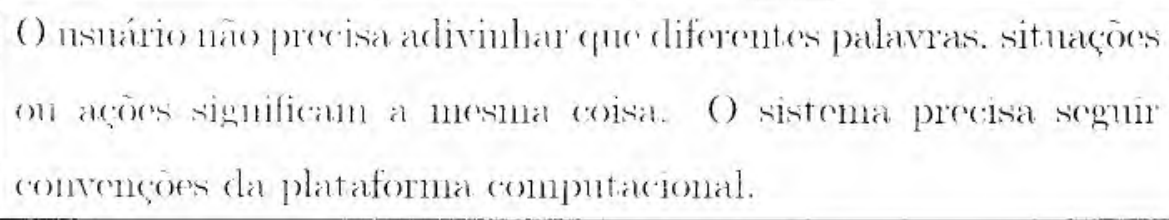 \\
\hline $\begin{array}{l}\text { Prevenção de } \\
\text { Erros }\end{array}$ & 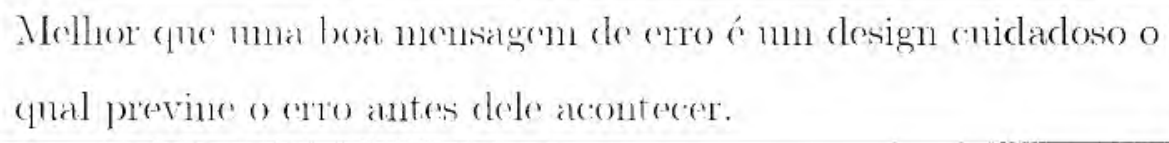 \\
\hline $\begin{array}{l}\text { Reconhecimento } \\
\text { ao Invés de Re- } \\
\text { lombranga }\end{array}$ & 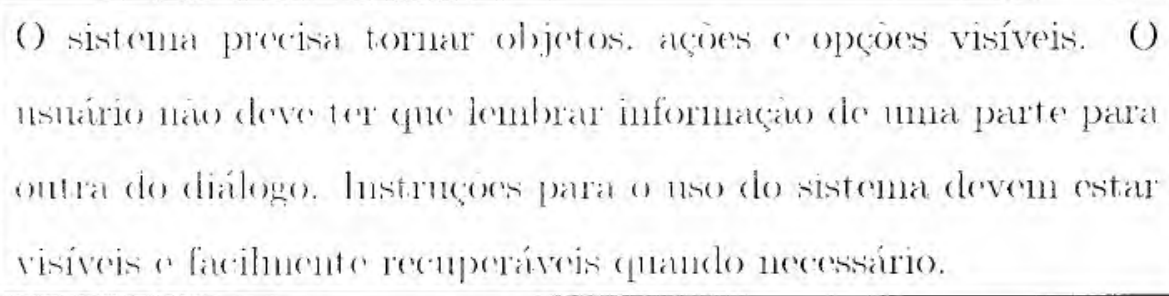 \\
\hline $\begin{array}{l}\text { Flexibilidade } \\
\text { e Eficiência de } \\
\text { Uso }\end{array}$ & 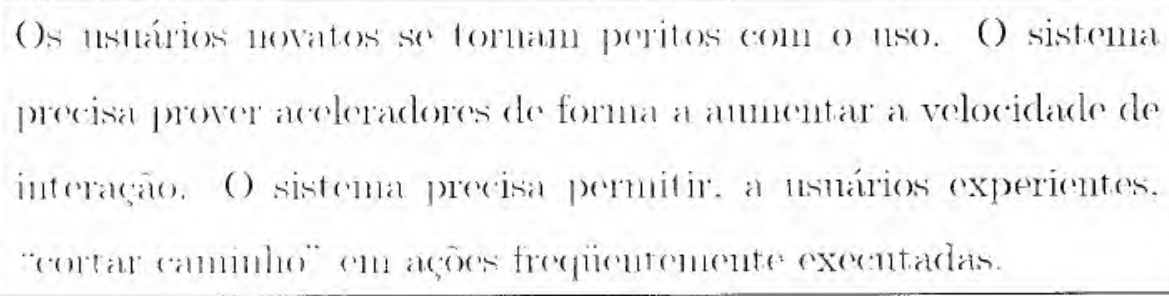 \\
\hline $\begin{array}{l}\text { Estética e De- } \\
\text { sign Minimalis- } \\
\text { ta }\end{array}$ & 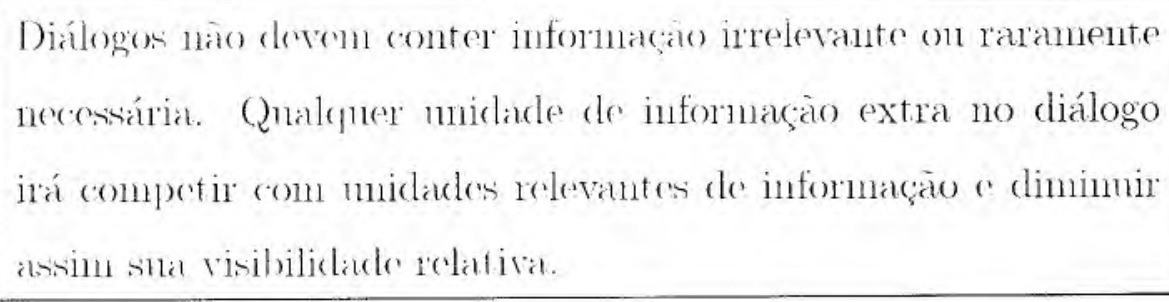 \\
\hline $\begin{array}{l}\text { Ajuda do } \\
\text { Usuário a } \\
\text { Reconhecer, } \\
\text { Diagnosticar e } \\
\text { Corrigir Erros }\end{array}$ & 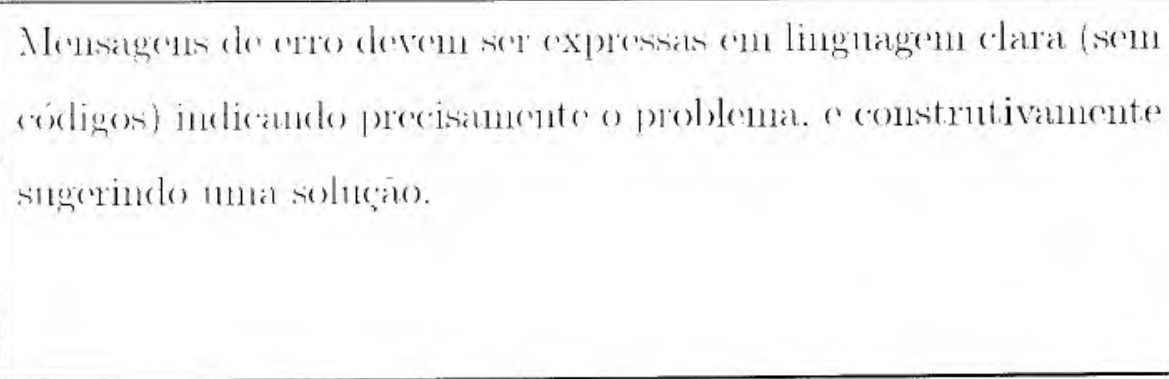 \\
\hline
\end{tabular}

(olltillta hat proximb pigina 


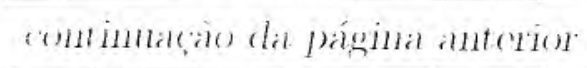

\begin{tabular}{|c|c|}
\hline \multicolumn{2}{|r|}{ Heurísticas de Usabilidade } \\
\hline $\begin{array}{l}\text { Ajurla e Docu- } \\
\text { mentação }\end{array}$ & 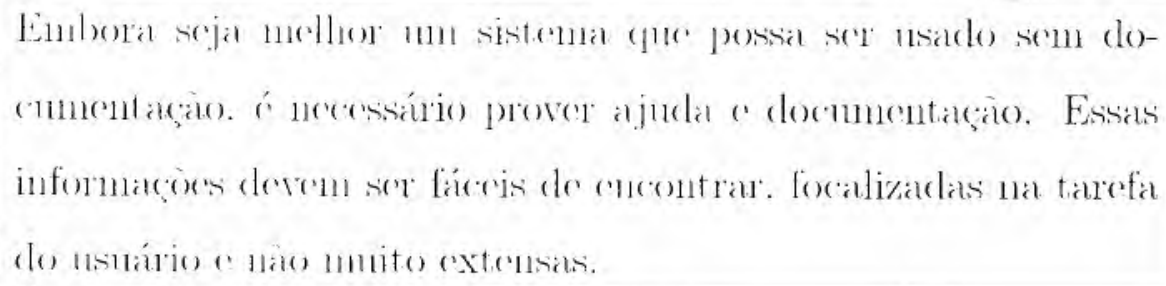 \\
\hline
\end{tabular}

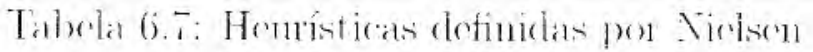

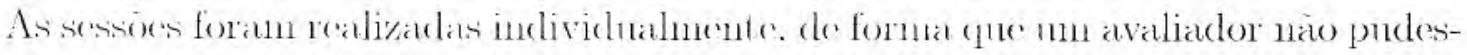

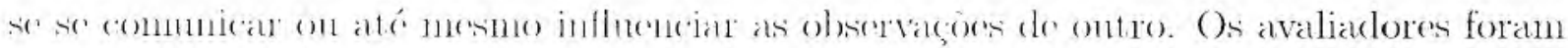

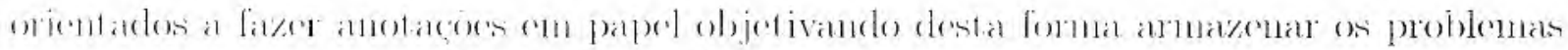

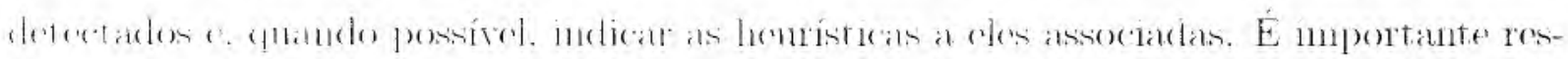

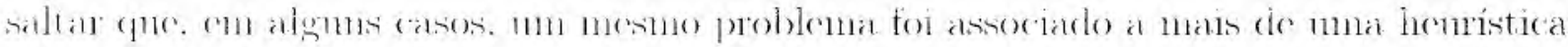

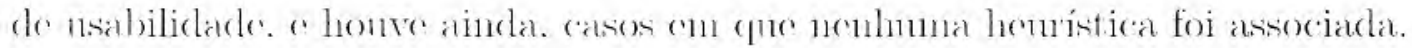

Esperial eniclardo foi tomado de forma que os resursos (software o hardware) utilizados durante as sessoes de avaliacion fossem equivalentes àcueles utilizados pelos nesuários reais an interagir (ont o sistemas.

\subsubsection{Pós-avaliação}

Na etaja de pós-avaliaço o avaliador exerenton análises quantitativas e qualitativas dos

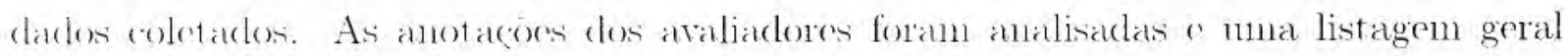

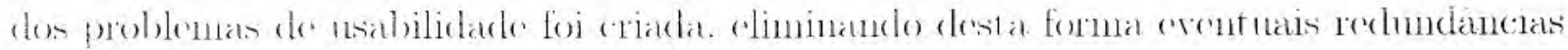

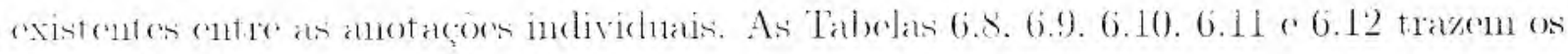

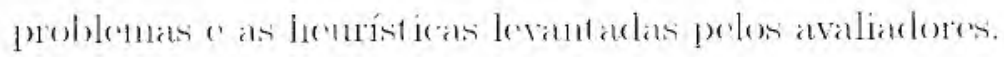




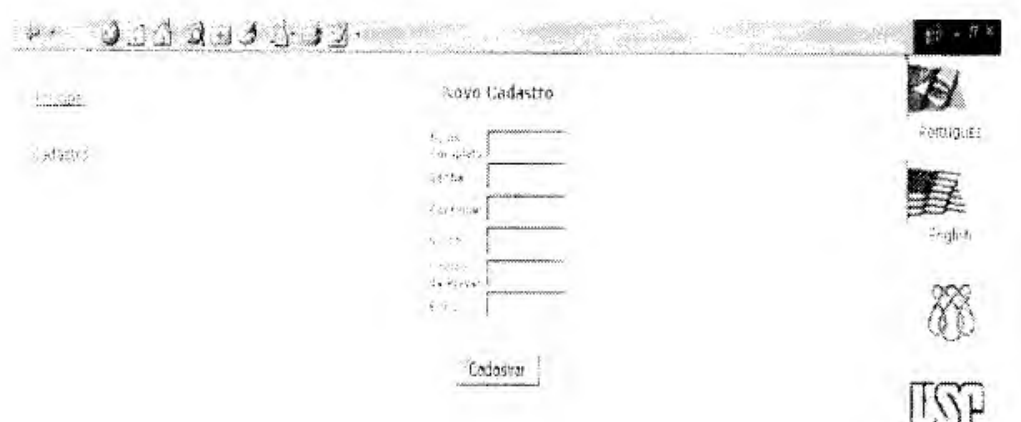

Figura 6.9: Interface do Cadastro.

Problemas Detectados na interface Cadustro (Figura 6.9)

\section{Heurística 7}

\section{Problema}

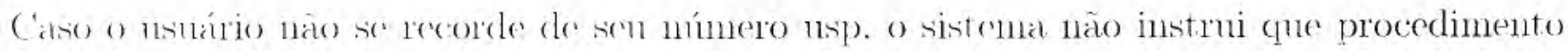
tollial:

\section{Solução}

Disponibilizar un help para inst ruir cono proceder nestes casost.

\section{Heurística $?$}

\section{Problema}

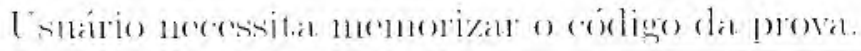

\section{Solução}

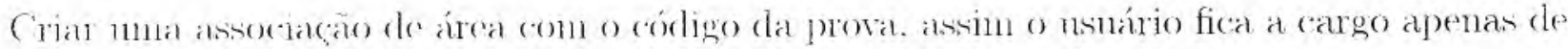
selecionar a que área pertence ro sistema se encarega de disponibilizar a prova adequada.

Heuristica 5, 10

\section{Problema}

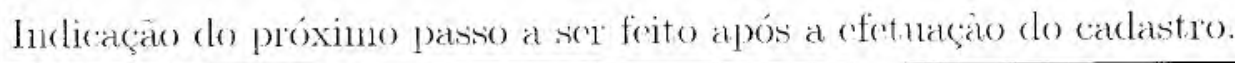




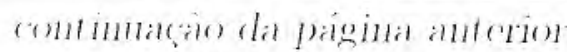

\section{Solução}

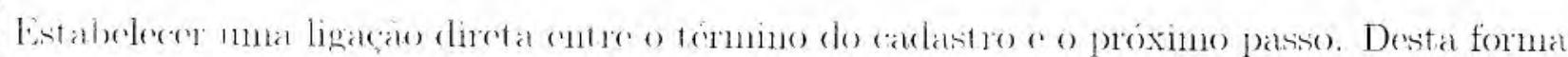

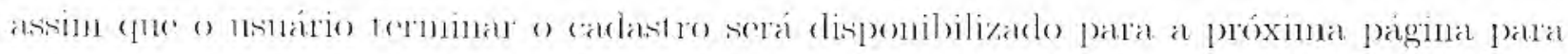

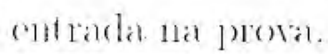

Heurística Nón há hromistica para assoriar

\section{Problema}

Văo sao recarregados os campos de "senha" e "Confirma senha" após nma mensagem de erro na interface de cadastro.

\section{Solução}

Recarregar estes canposs.

\section{Heurística \&}

\section{Problema}

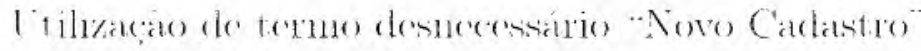

\section{Solução}

Utilizar sonente o terme Carlastro.

\section{Heurística $\&$}

\section{Problema}

Existencia de ancota patra a paiginal de caulastro.

\section{Solução}

filininat rata ancola.

\section{Heurística 8}

\section{Problema}

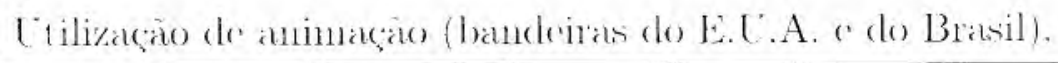

\section{Solução}

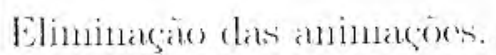

\section{Heurística?}




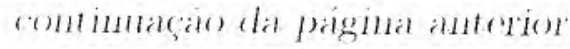

\section{Problema}

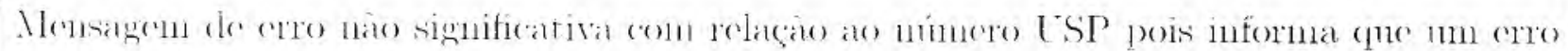
ocorten mas náo informa qual.

\section{Solıção}

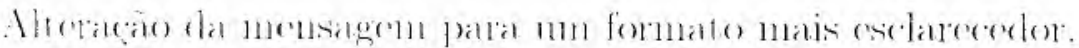

\section{Heurística 1}

\section{Problema}

Após a confiumarào de cadastro há a permanoneda da mesma interface.

\section{Solução}

Autonatizar o processo de disponibilizar a próxina interface para cutrada na prova.

\section{Heurística !l}

\section{Problema}

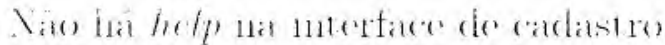

\section{Solıção}

D)isponibilizar help na interfines de cardastro.

Heurística $\delta, 8$

\section{Problema}

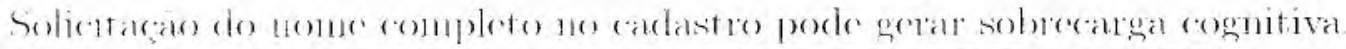

\section{Solução}

Criacaro clo $111 \mathrm{n}$ identificador monor do tipo user name.

\section{Heurística ?}

\section{Problema}

Cilizacào do trunos "Principal" " "Logon"

\section{Solução}

Sulstituiçite de termos.

\section{Heurística 5}

\section{Problema}

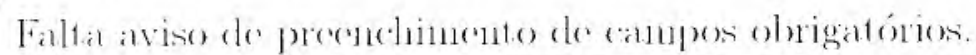


(omtimascalo da página anterion

\section{Solução}

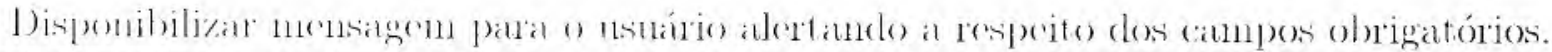

Tabela 6.8: Relacaro de problemas na interface do Caulas-

t.10)

Problemas Detectados na interface Grueral Instrurtuons.s (Figura 6.10)

\section{Heurística 1}

\section{Problema}

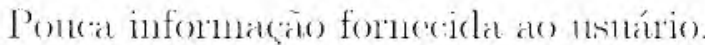

\section{Solução}

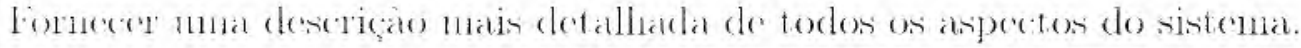

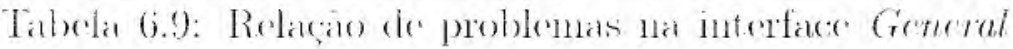

Insturutions

\section{Problemas Detectados na interface Conlenls (Figura 6.11)}

\section{Heurística}

\section{Problema}

Pomeo enfogne para o conterílo rlestia interface.

\section{Solıção}

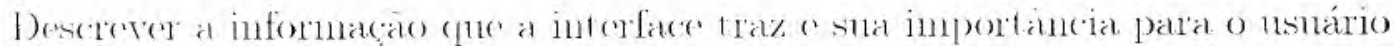

\section{Heurística ?}

\section{Problema}

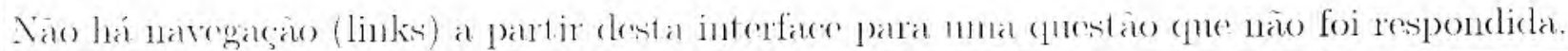

\section{Solução}

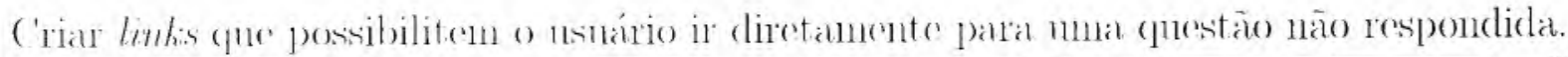

Heuristica /, ,

(ontinna na próxima página 
(ontintargue da prigina anterior

\section{Problema}

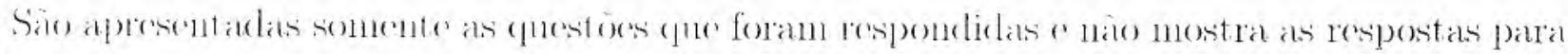

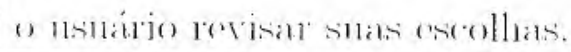

\section{Solução}

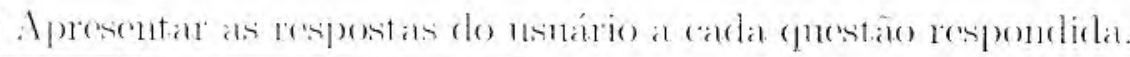

Tabela 6.10: Relacaro do problentas na intorface Contents

Problemas Detectados nas interfaces das P'art I. Part .. Part 3. ...

\section{Heuristica 10}

\section{Problema}

Näo há help associado aos botós de instructions. explanation. teat e questions.

\section{Soluçāo}

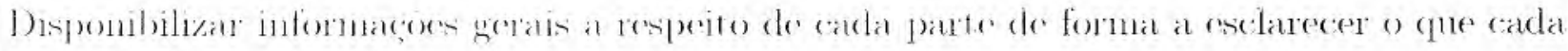

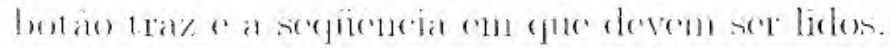

\section{Heurística $7, ;$}

\section{Problema}

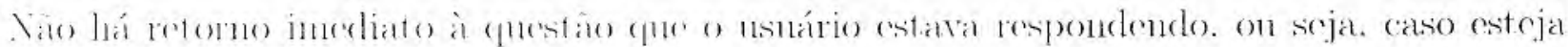

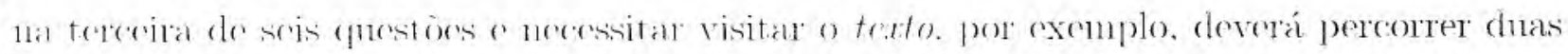

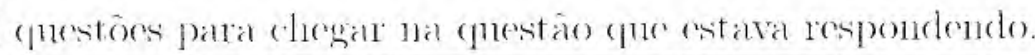

\section{Solução}

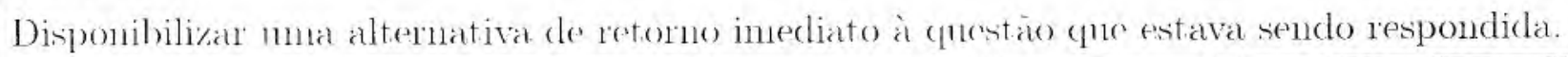

\section{Heurística 8}

\section{Problema}

Há guestoes enu que nate existe informacion na interface "explanation" e a razào deste fato Hàto á informadal ato mistário.

\section{Solução}

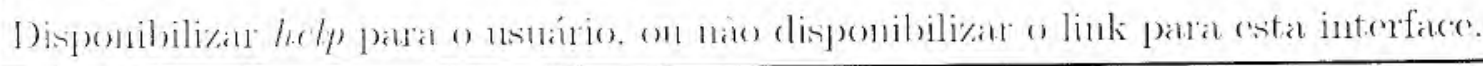




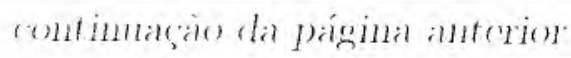

\section{Heurística 6}

\section{Problema}

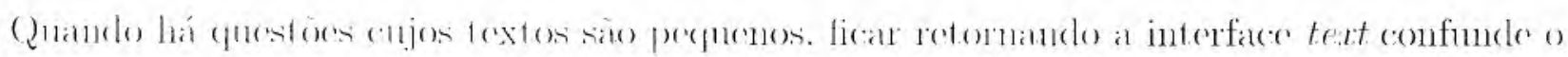
Mstrition.

\section{Solução}

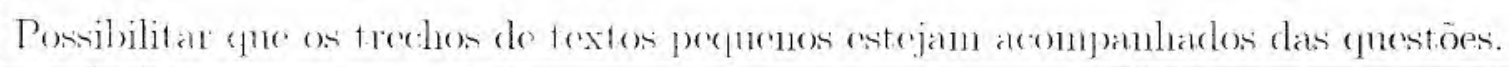

\section{Heurística 4}

\section{Problema}

Limkis de Barkie Next variann de posicaro on algunnas interfaces.

\section{Solução}

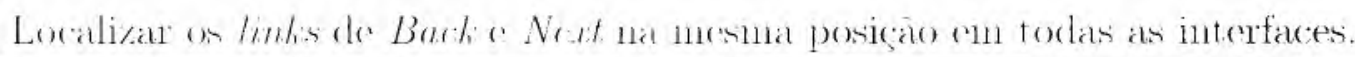

\section{Heurística 4}

\section{Problema}

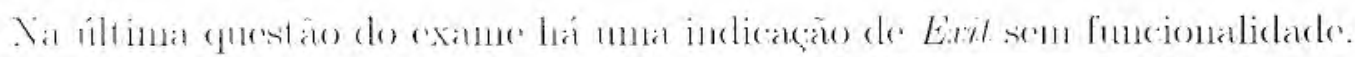

\section{Solução}

Tornat a indicamato do Ent funcional.

\section{Heurística ;}

\section{Problema}

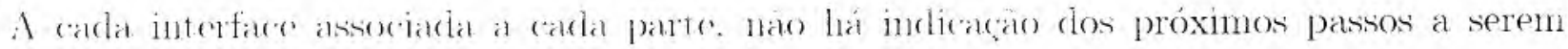
sergniclos

\section{Solução}

Disponibilizar a informacáto de gltad o proximo passo a tomar.

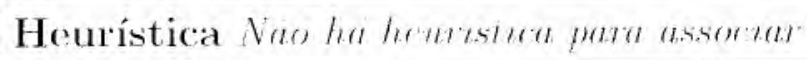

\section{Problema}

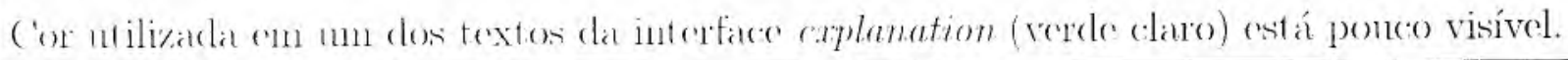

\section{Solução}

Alscrar a cor.

\section{Heurística of}


(ont inhaço da página antrexon

\section{Problema}

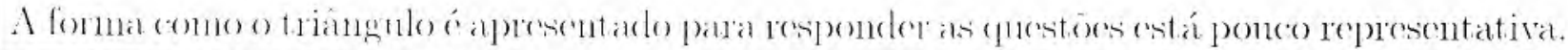

\section{Solução}

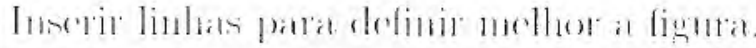

\section{Heurística o}

\section{Problema}

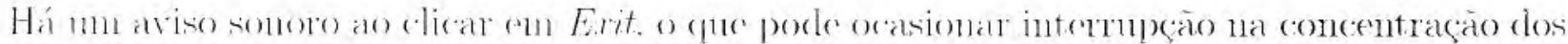
outros llstiritios.

\section{Solução}

Eliminar o ariso sonoro.

\section{Heurística ;}

\section{Problema}

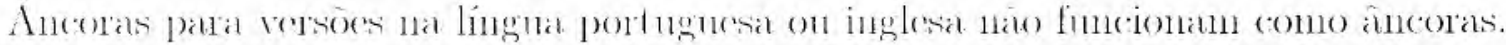

\section{Solução}

Elimina инсоны.

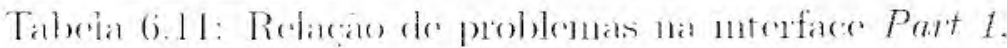

Part ?. P'art i. ...

\section{Problemas Detectados na interface Resultados}

\section{Heurística l}

\section{Problema}

Vilo hat aviso patra encemal o sistemat.

\section{Solução}

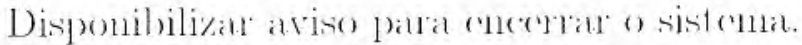

Heurística is \&

\section{Problema}

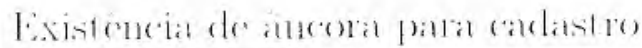




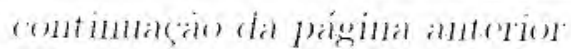

\section{Solıção}

Elininar ancolat.

Talbela 6.12: Relacio de problemas na interface Resultedos

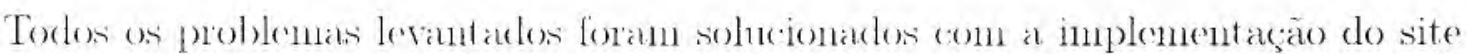

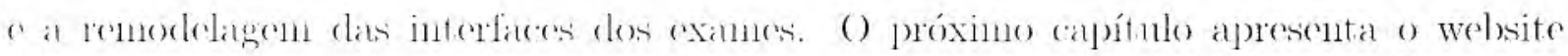
('AP'TEAP'. 
General Instructions
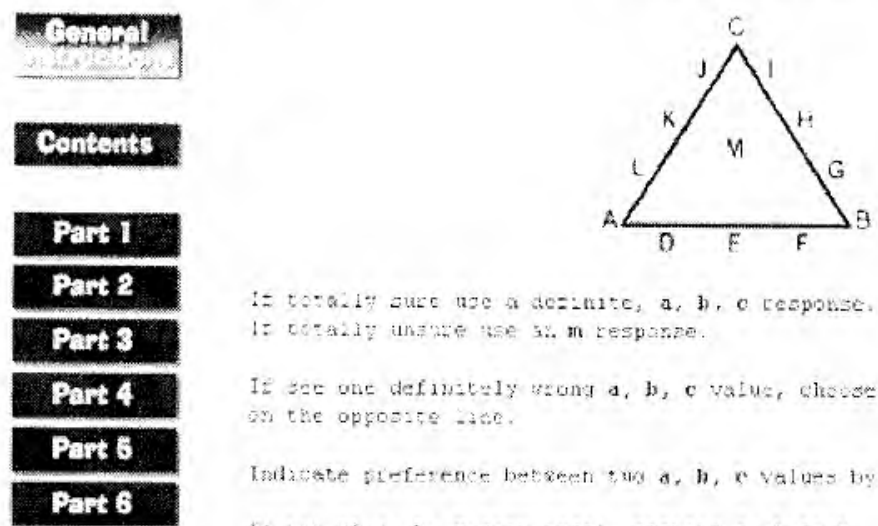

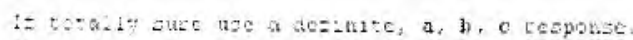

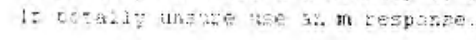

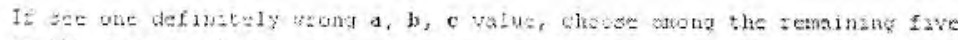
ar the opposts -xes

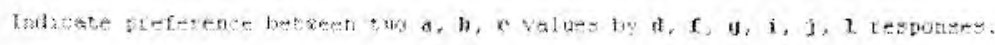

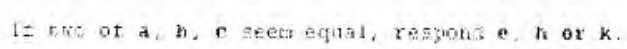

\section{ExI}

Next,

Fignua 6.10: Interface de General Instructions.

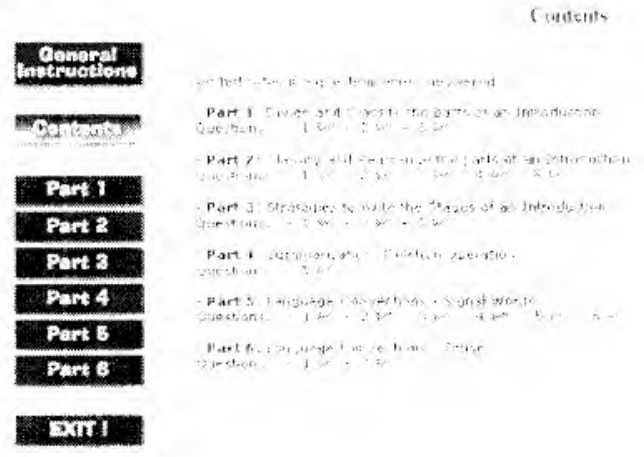

Back

Next,

Fignua 6.11: Interface de Contents. 


\section{Capítulo 7}

\section{Um sistema baseado na Web para o}

\section{EPI para o mestrado do ICMC-USP}

\subsection{O site CAPTEAP}

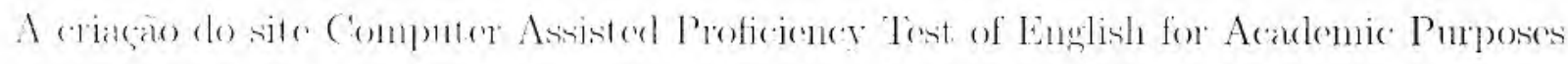

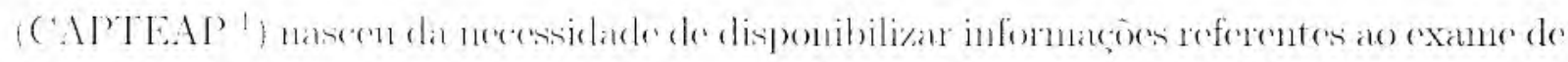

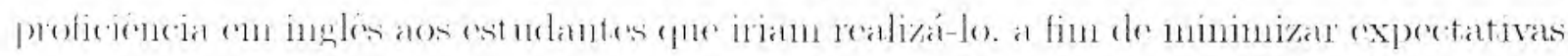

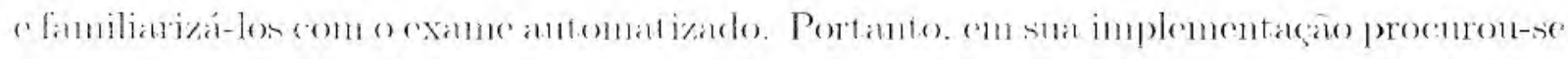

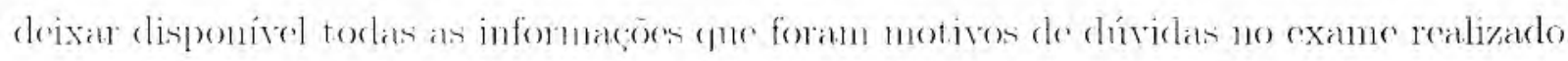
enu abril de 2001. A Figna 7.1 mostrat a interface principal do site. A interface mostrada na Figura 7.1 apresenta links an forma de botoes distribuídos en torno de 1 me círculo que

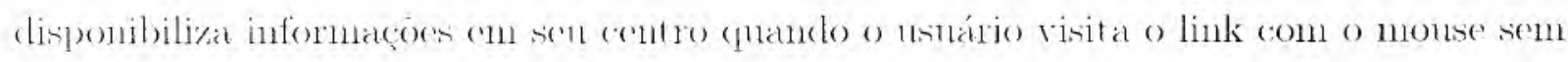

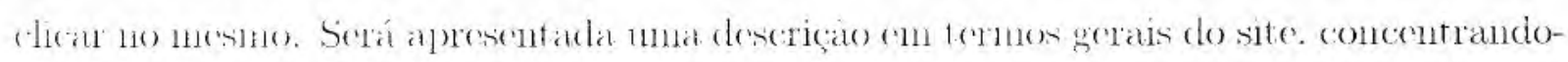

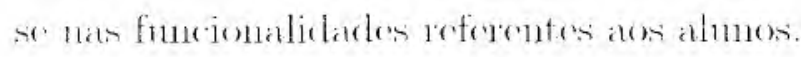

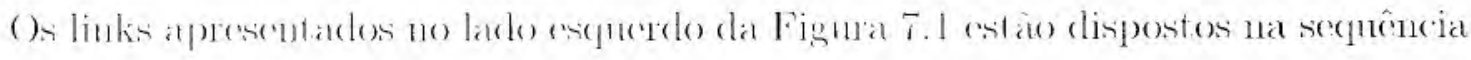

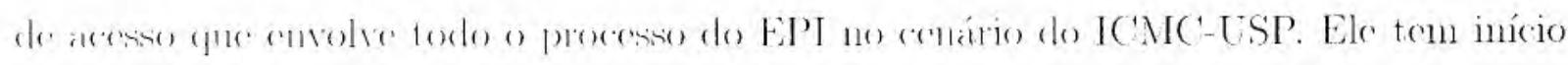

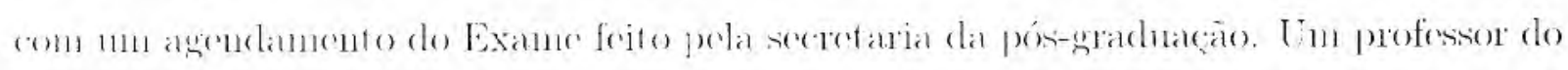

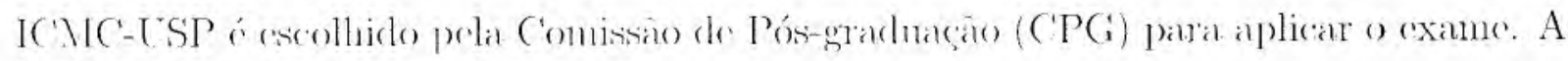

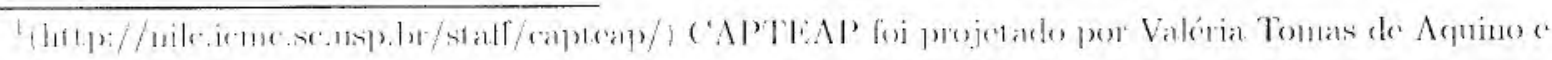

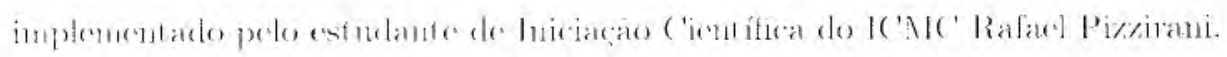




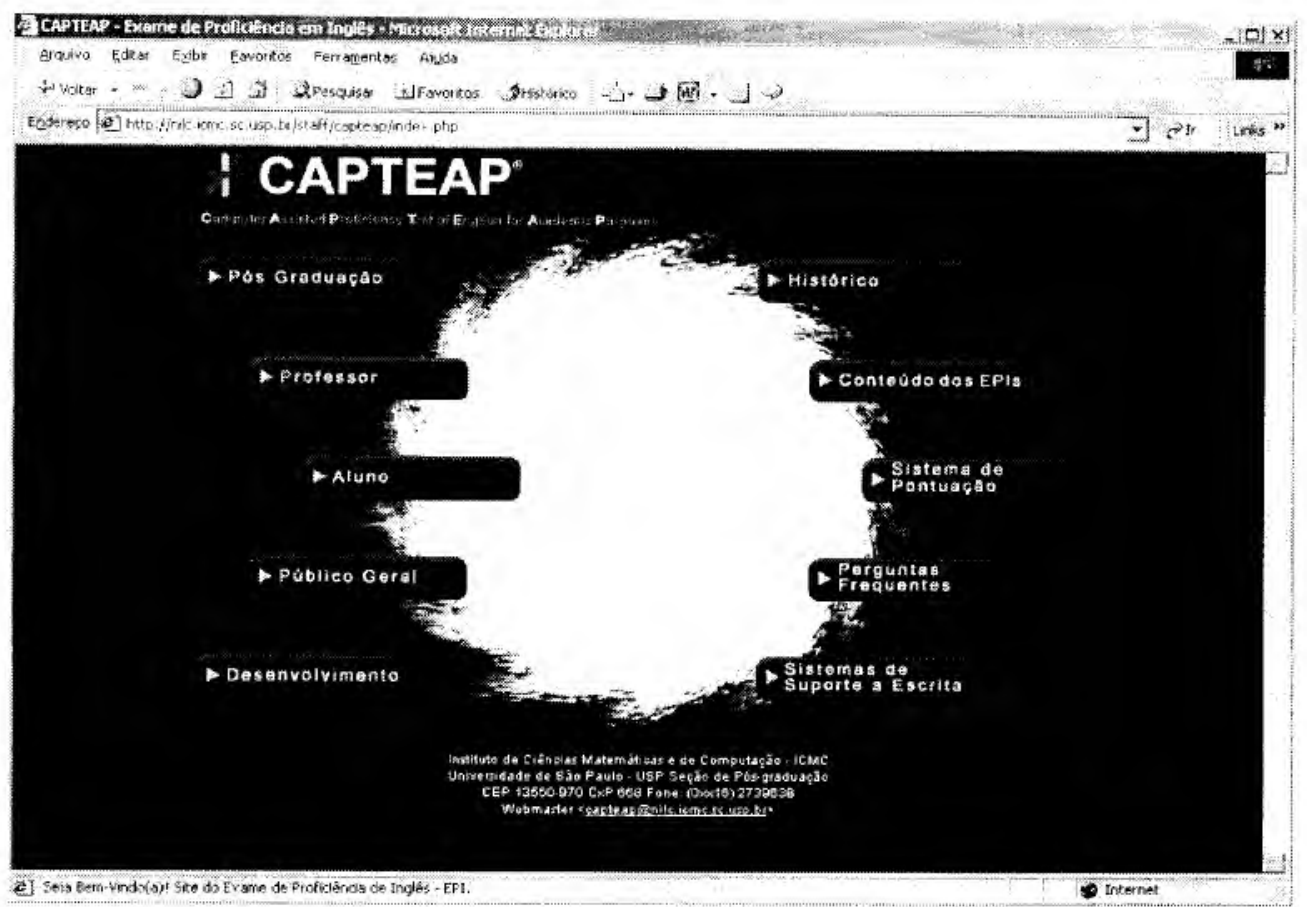

Fignua 7.1: Interface P'riucipal do Sire CAPTEAP.

serectaria da pós faz o agendamento do exame com os seguintes dados:

- Vome do profersor responsível pelo EPI do semestre:

- Datar der aplicaçaro do EPl:

- Nímero máxino de alunos por árar (Estatística. Natemática Computacional e Comprita(ca):

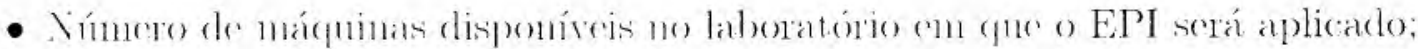

- Possíveis horários para a apliexcouo do EPl.

() agendanente do exane feito pela secretaria da pós inplica na permissáo para edicaro da prova pelo professor nomeado para aplicar o EP'. A criaçăo do exanne inicia-se

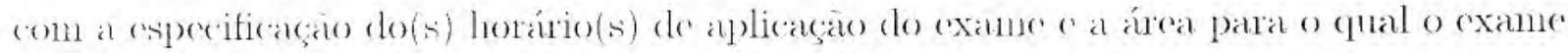

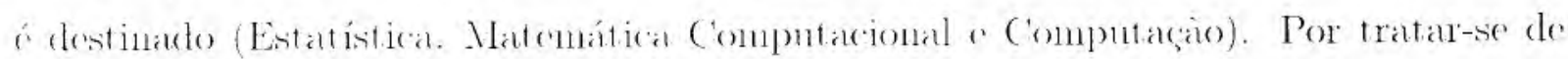

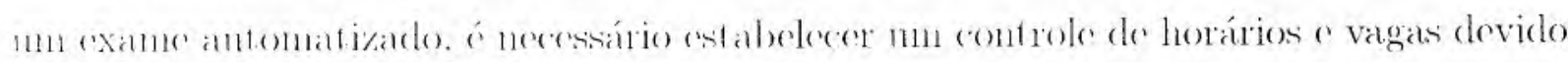

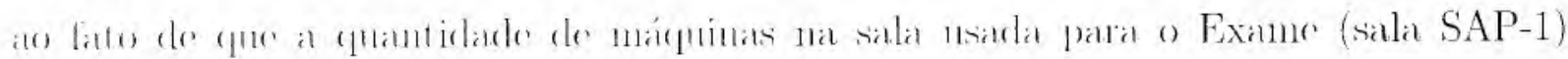




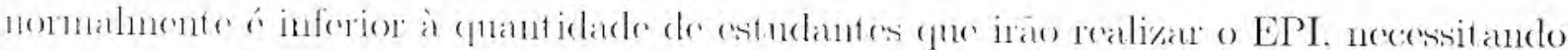

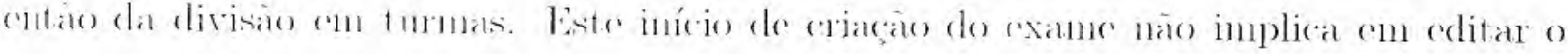

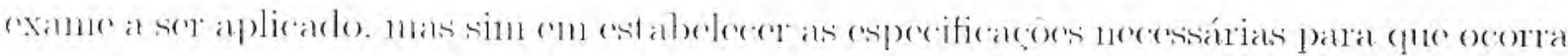

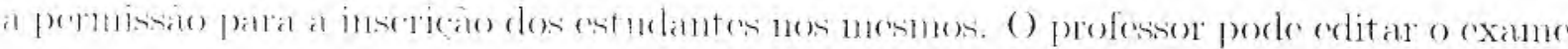

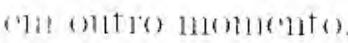

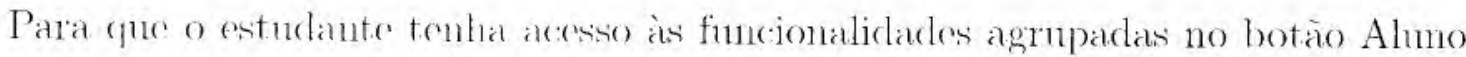
(Fignua 7.3). ć necessádio que cle se andastere no sistema. Após preencher os dados nesta

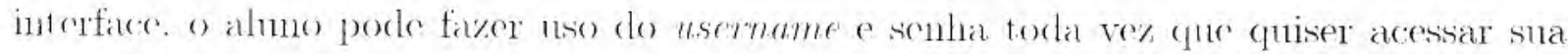
ára. Por necio da interface mostrabla na Fignua 7.30 aluno fay a entrada dos seguintes

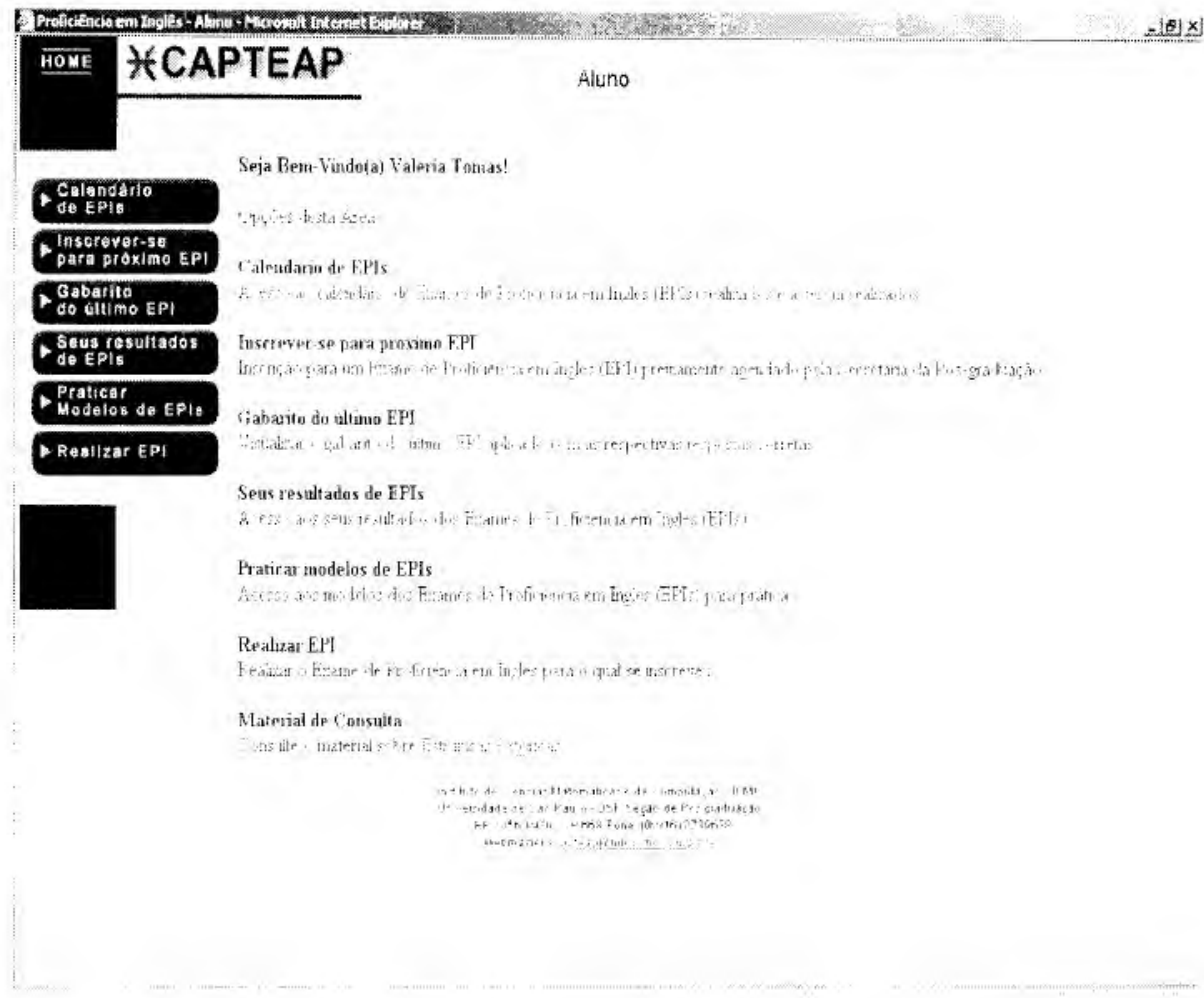

Fignara T.2: Interface 1 pertinente ans alunes do ICAC-USP para acesso.

diukes:

- Vomer (ompleto:

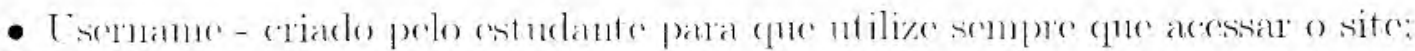

- Área - csecolliedar a partir de mula lista disponibilizadar pelo site. A área vincula todas 


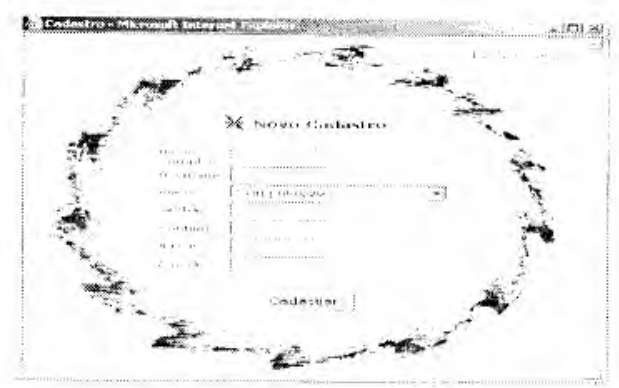

Fignea 7.3: Interface 1 pertinente anos alunes do ICMC-USP para cadastro.

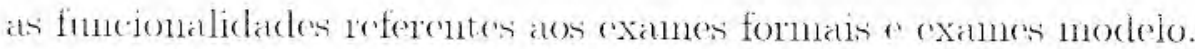

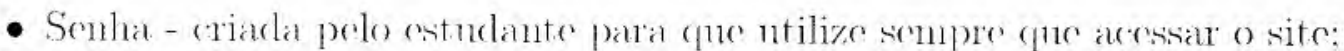

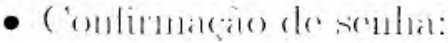

- Vínero L'SI':

- F-mail.

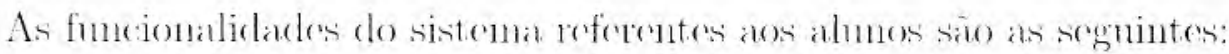

Calendário de EPIs. Acrsso ao calendário do Fxames do Proficiencia enu Inglês

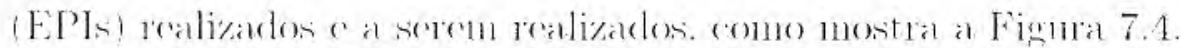

Como mostra a Fignea 7.4. sao disponibilizadas nuformacoes de data e professor responsável peło EPI a ser aplicado. Os EPIs já aplicarlos também estarāo relacionados nestat mestuma intertare.

Inscrever-se para próximo EPI. Inscriçăo para nun Exame de Proficiência em Ingles (EPI) previanente agendaulo pela Secretaria da Pós-graduaçào (Figura 7.5). Na interface mostrada na Figma 7.5 san disponibilizadas datas referentes a aplicagăo dos

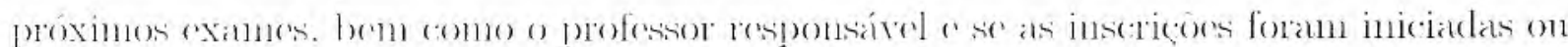

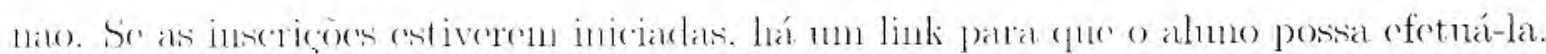

Gabarito do último EPI. Permite vistlalizar o gallarito do último EPI aplicado

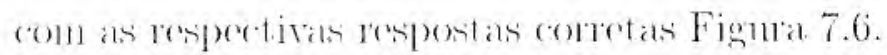

Seus resultados de EPIs. Aresso anos resultarlos do estudante nos Exames de Proficioncia rn Inglós (EPTs) (Fignat 7.7). 


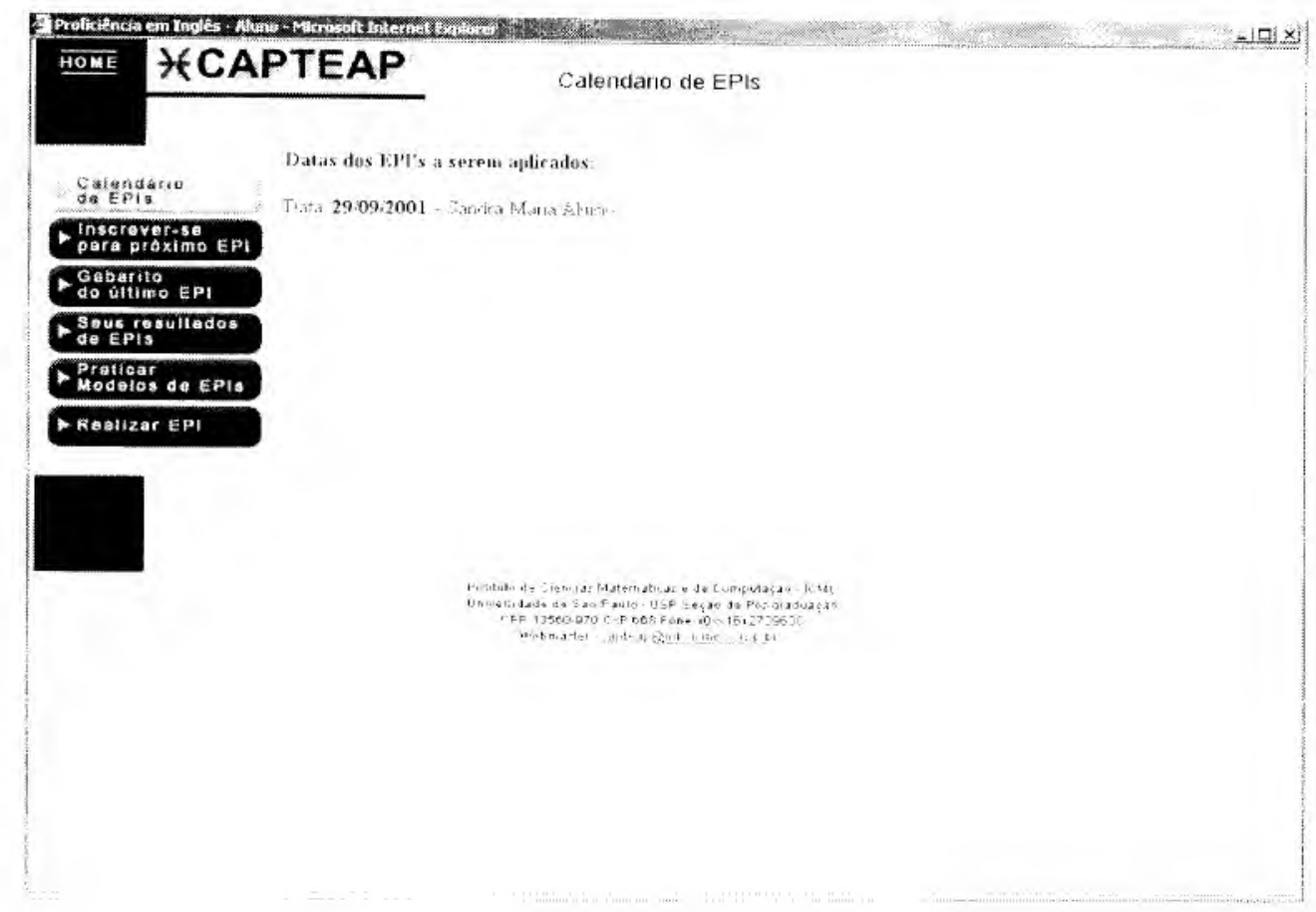

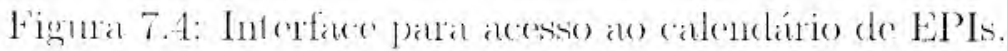

Praticar modelos de EPIs. () acesso ans modelos dos Fxames de Proficiencia om Ingles (EPIs) para prática é feito por meio da interface mostrada na Figura 7.8. Como mostra a Fignua 7.8. sado aprescentados os links para os exames disponiveis para prática.

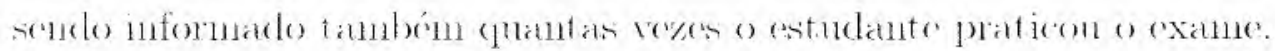

Realizar EPI. () EI'I propriannento dito.

Alón dos EPls formais diferirem dos modedos conn rolagaio anos textos cientificos.

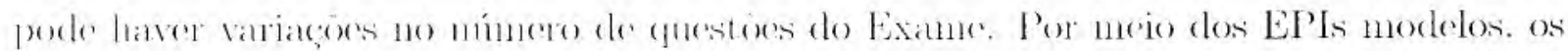

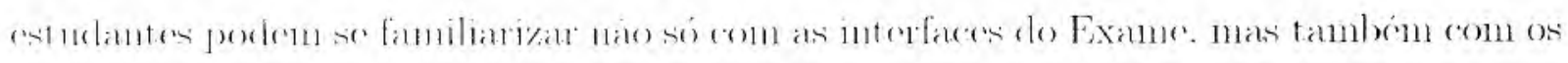

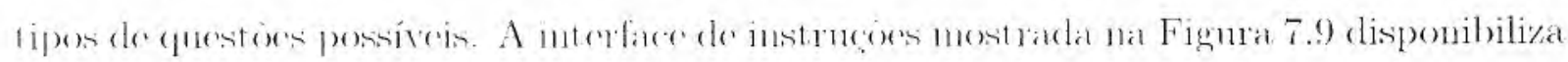

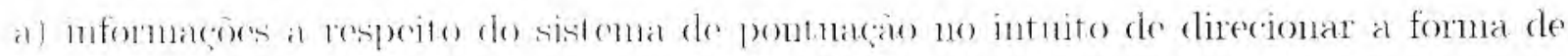

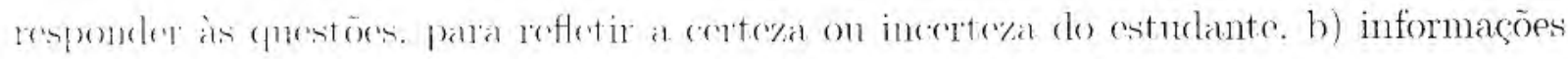
a respecito do software om termos de procedimentos a serem eritados a (c) informaçóes solope os acosios a partir dos botõos que a interface possui. As informaçoes disponíveis nesta interfacer foran alteradas para atender 1 m dos problemas levantados na avaliaçào

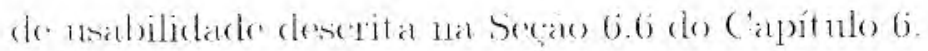

A Figura 7.10 mostra a interface (1ue o estuclante dispoe para fazer un acompanhat- 


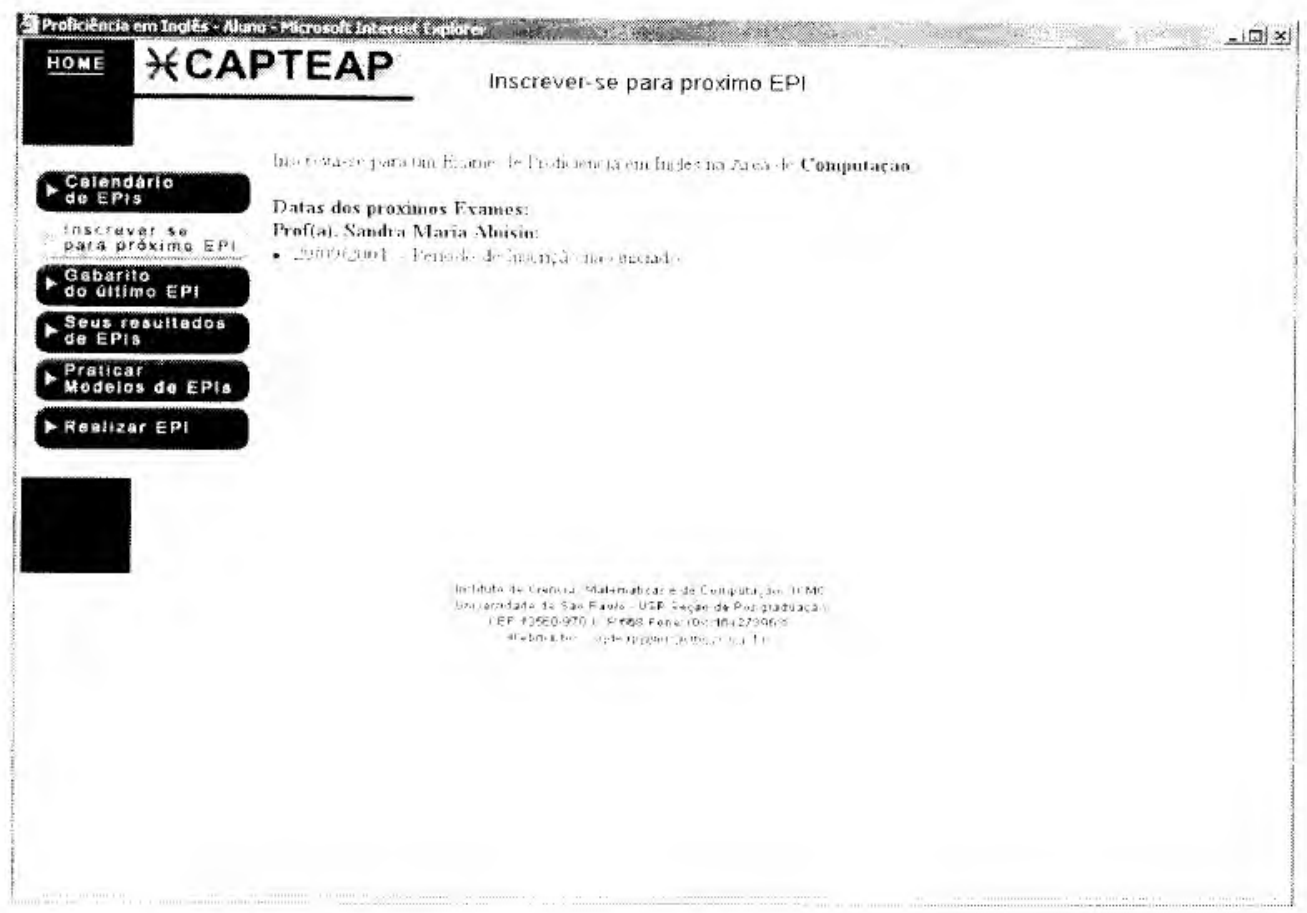

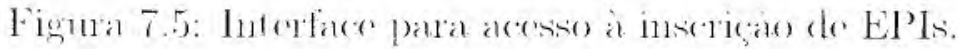

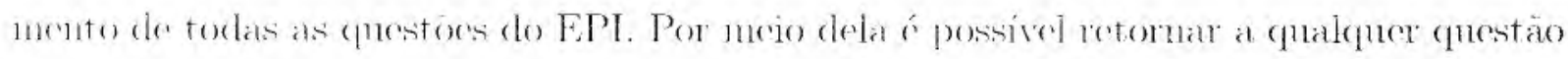

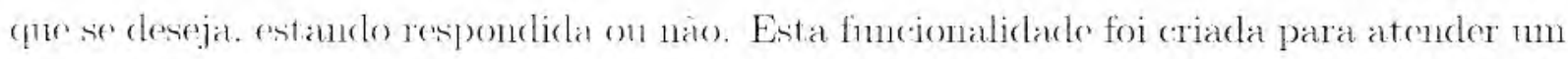

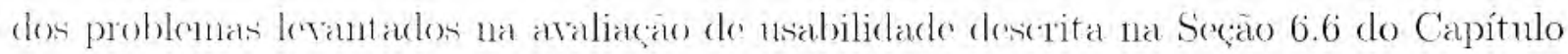
(j.

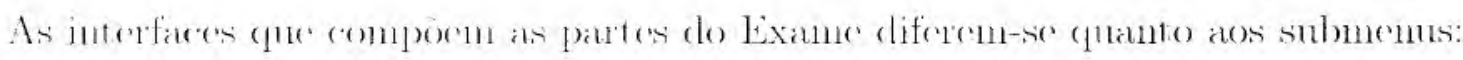

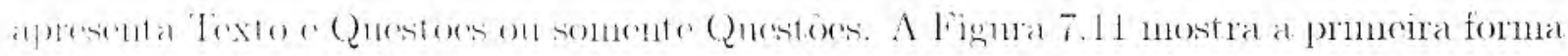

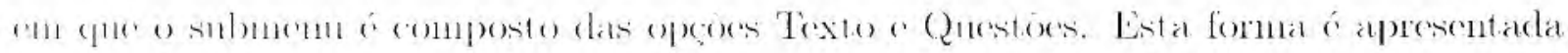

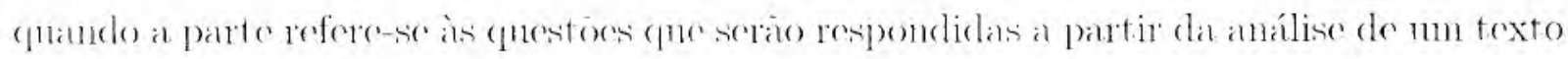

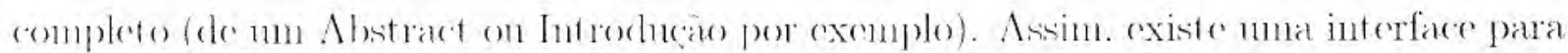

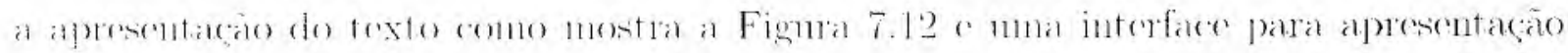

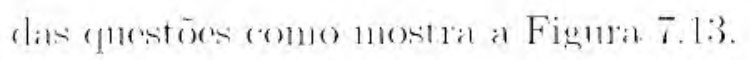

A segmurla forma. mostrada ra Figma 7.14, apresenta um submenu com apelias a

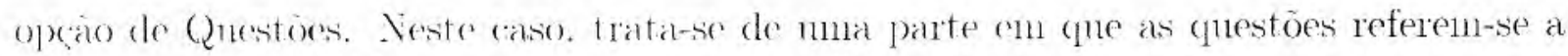

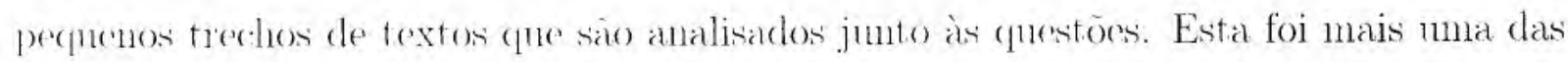

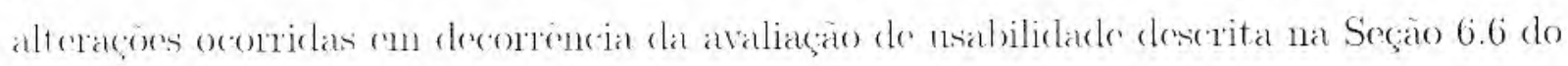

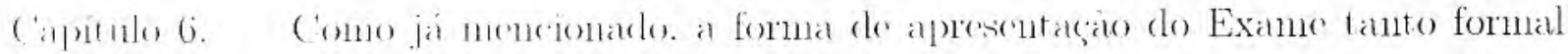




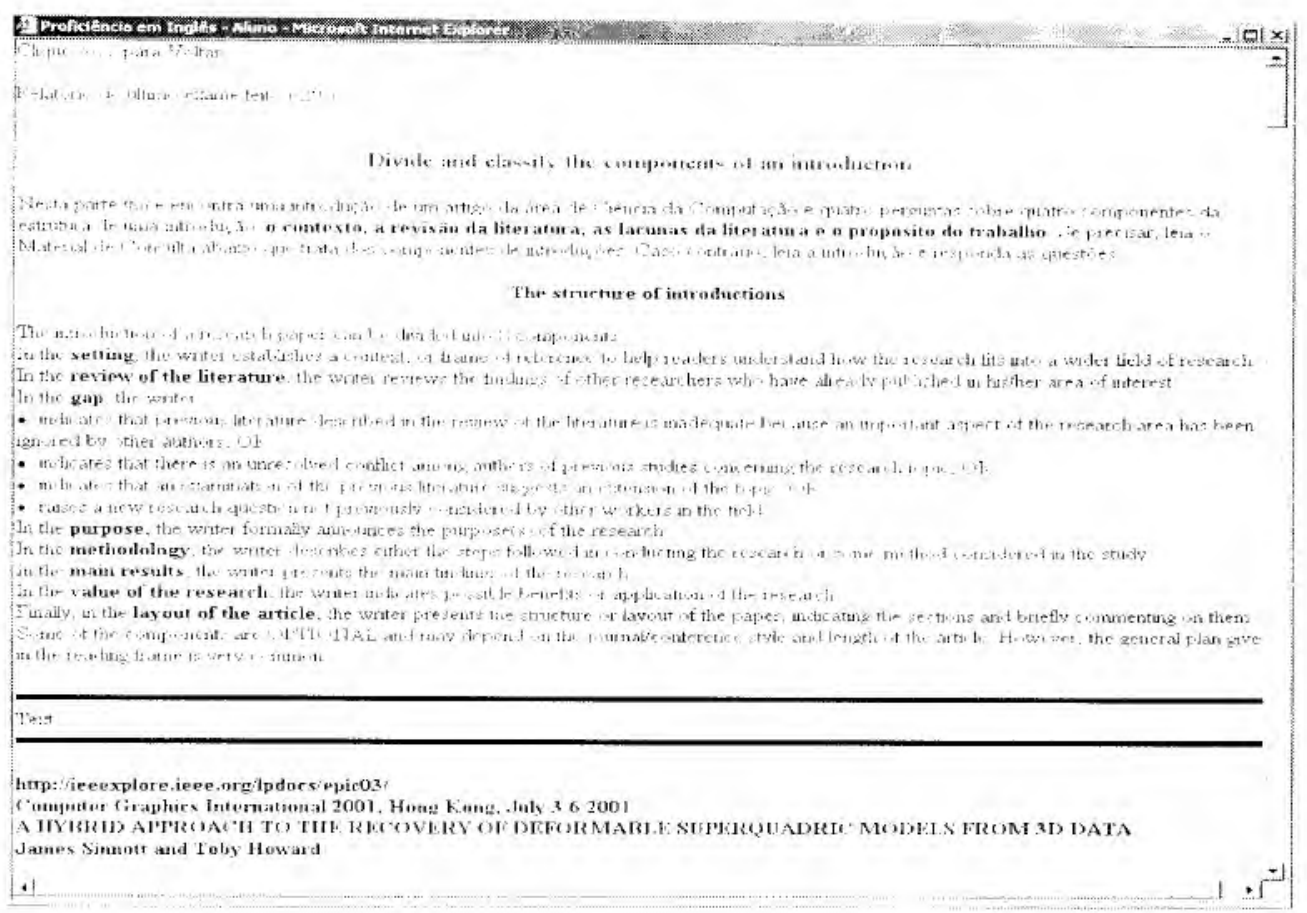

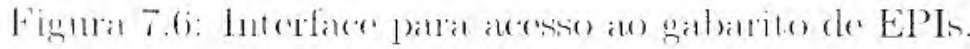

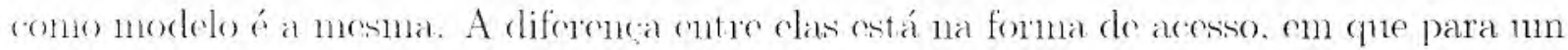

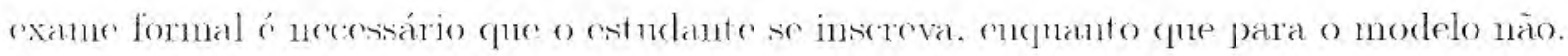
A liberasáa das permissoes das provas formais ó feita pelo administrador do sistema no nunnento do Exanne.

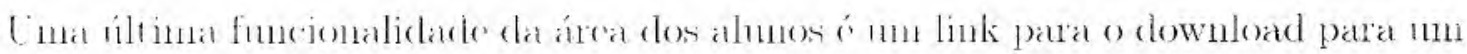

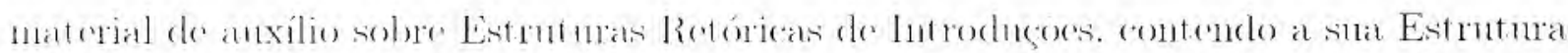

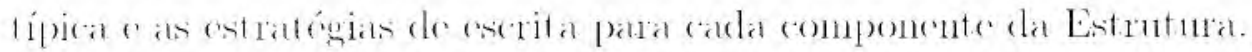

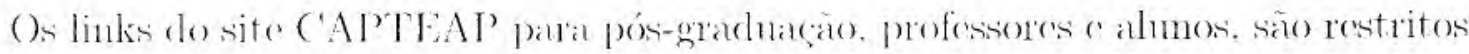

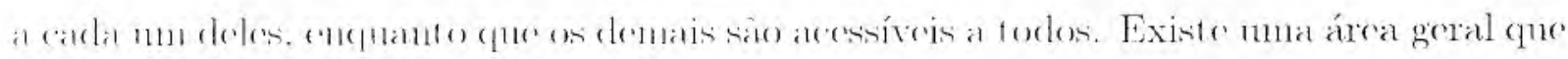

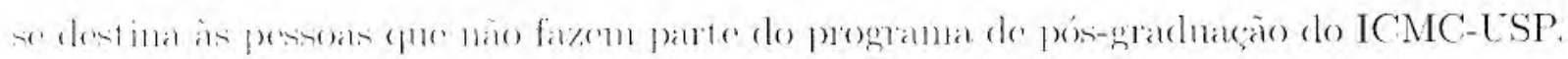
Cono nustra a Figura 7.16. a interfares para este publico traz wna funcionalidade que pernite a prática de mu modelo de FPI. disponibilizando oxame aos fut nuros ingressantes no progranta de mestrado do ICMC-LSP'.

Os ontros links disponíveis para ancesso geral a part ir da interface principal sado:

Desenvolvedores - Disponibiliza informacoes a reapeito das pessoas envolvidas 10 pojeto do sistema CAP'T'EAl'. 


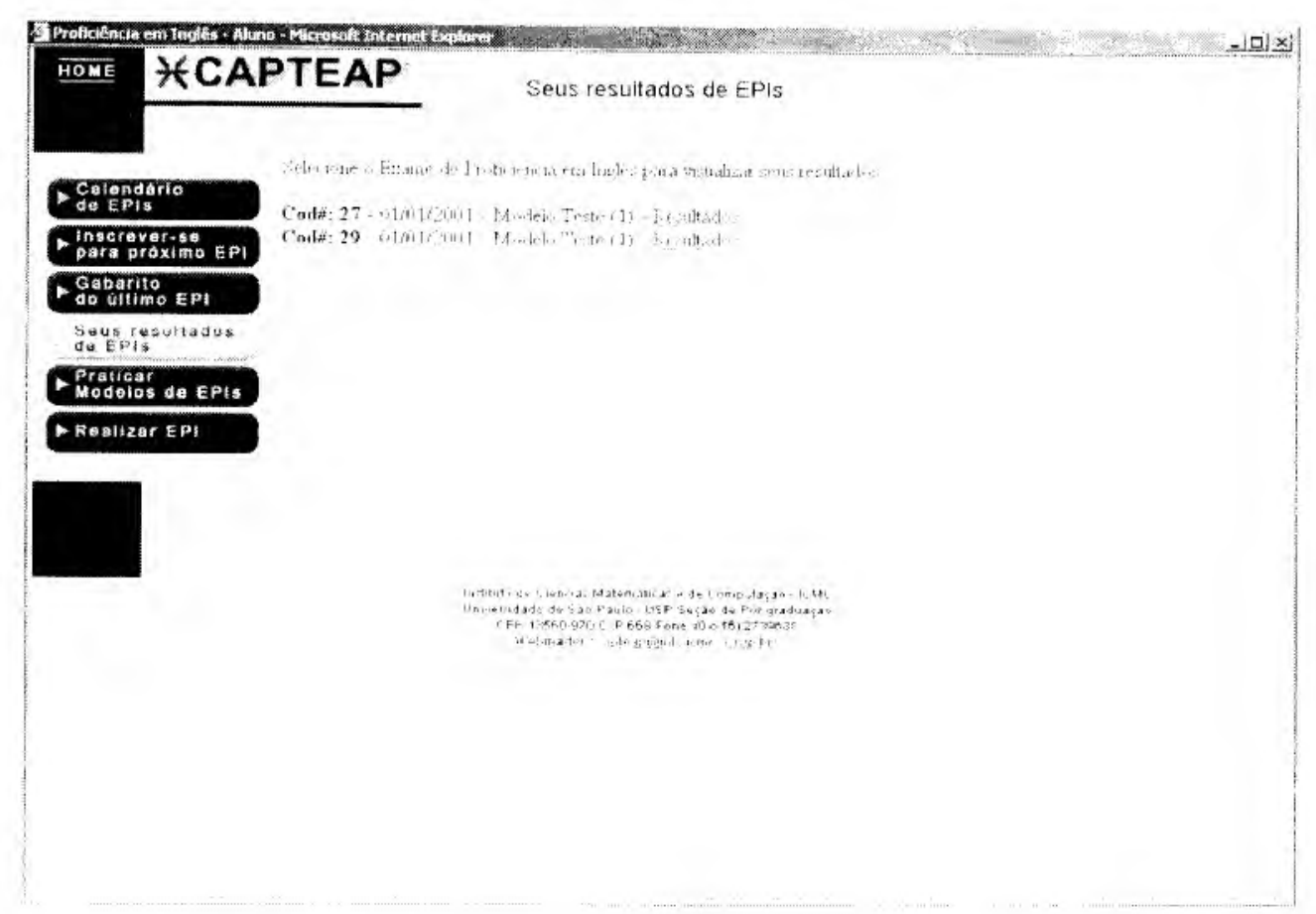

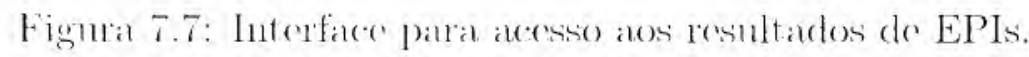

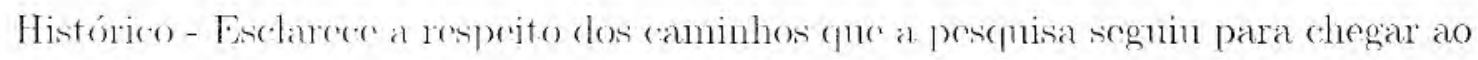
desemolvinuento de inn EPI informatisado.

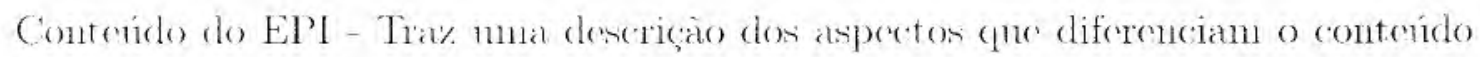

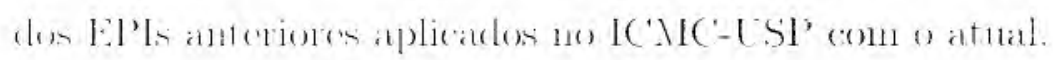

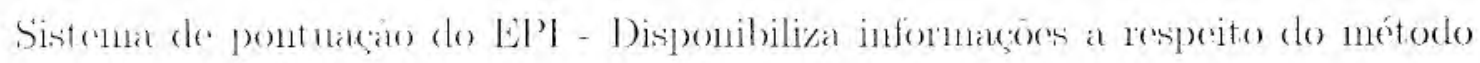

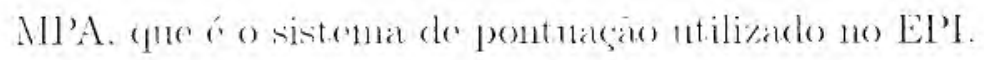

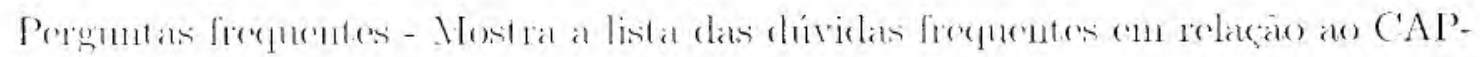

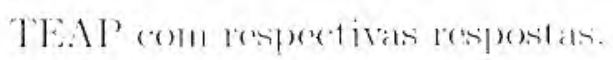

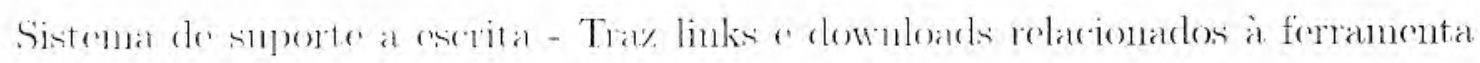
de crítica do Ambiente AXADELS (Alnísio ot al.. 2001) pana que os interessaddos possam

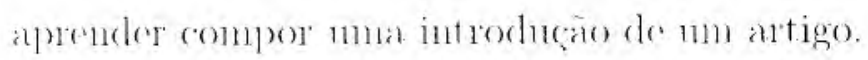

Todas as funciomalidades dispostas no site foram fruto do desejo de atender as urecessicladen dos estudantes observadas ao longo dos vários testes feitos com o sistema. 


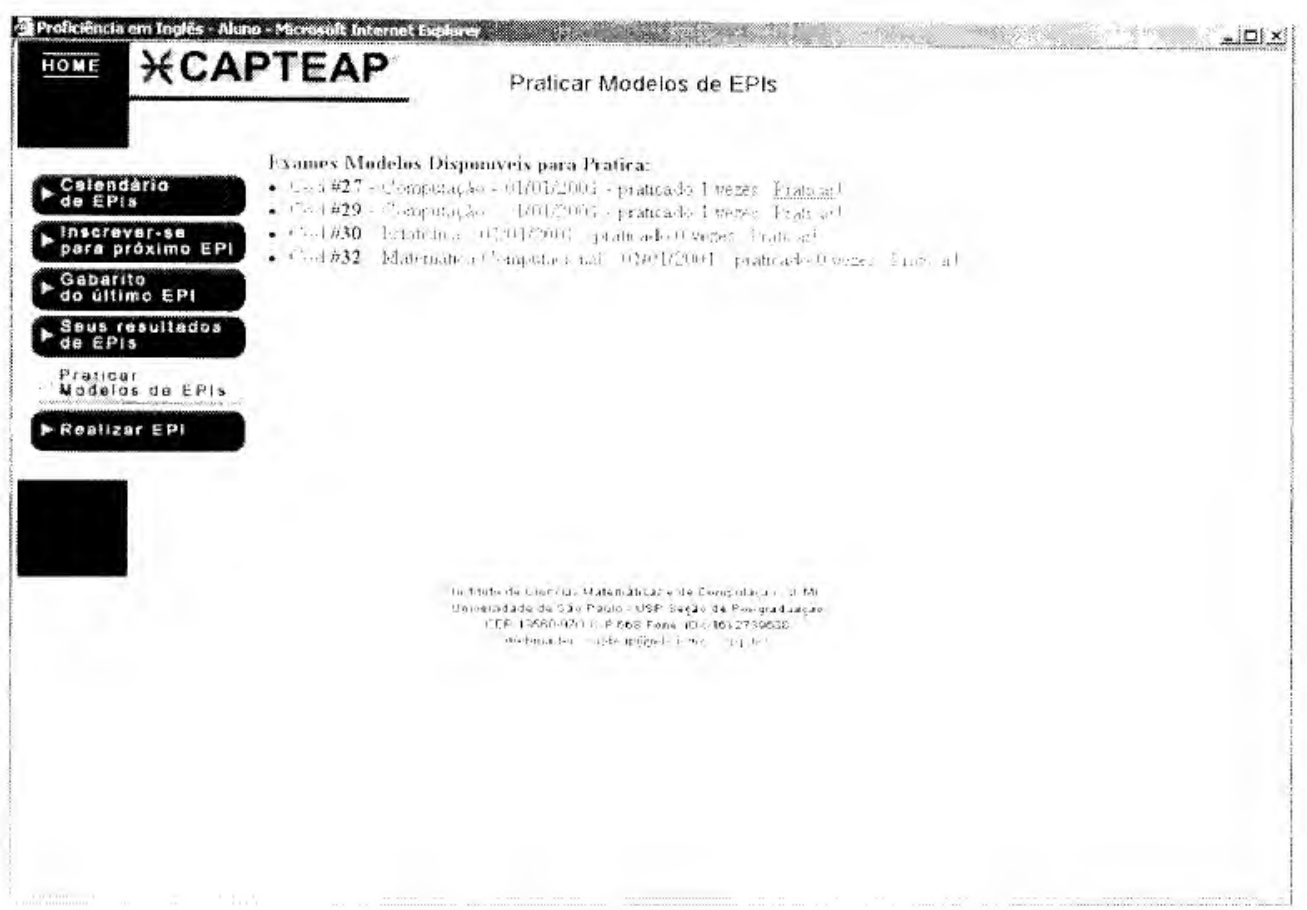

Fignua 7.8: Interface para aresso a pritica de EPIs.

\subsection{Arquitetura do sistema}

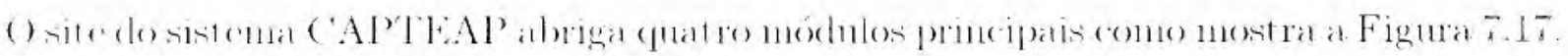

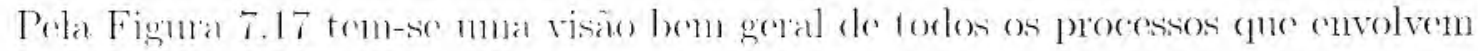

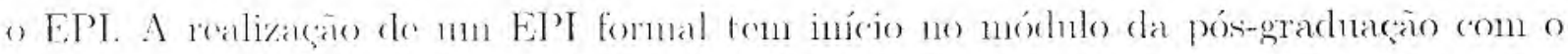

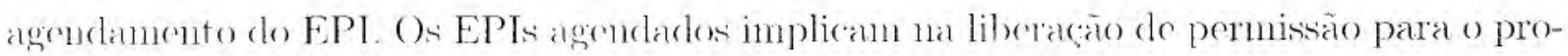

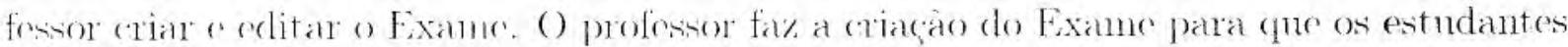

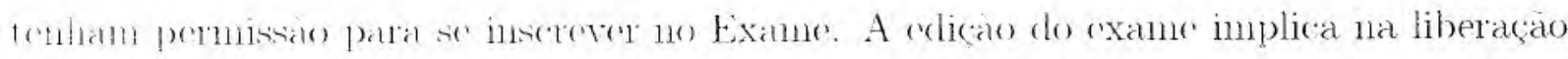
de permissáo para realizaça do mesno feita pelo administrador no dia/hora do EPI. O

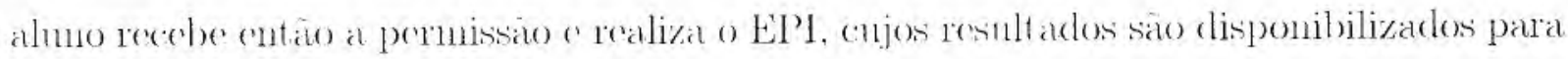

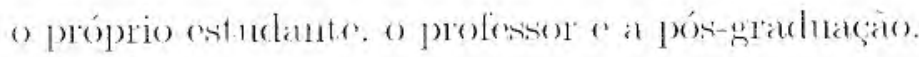




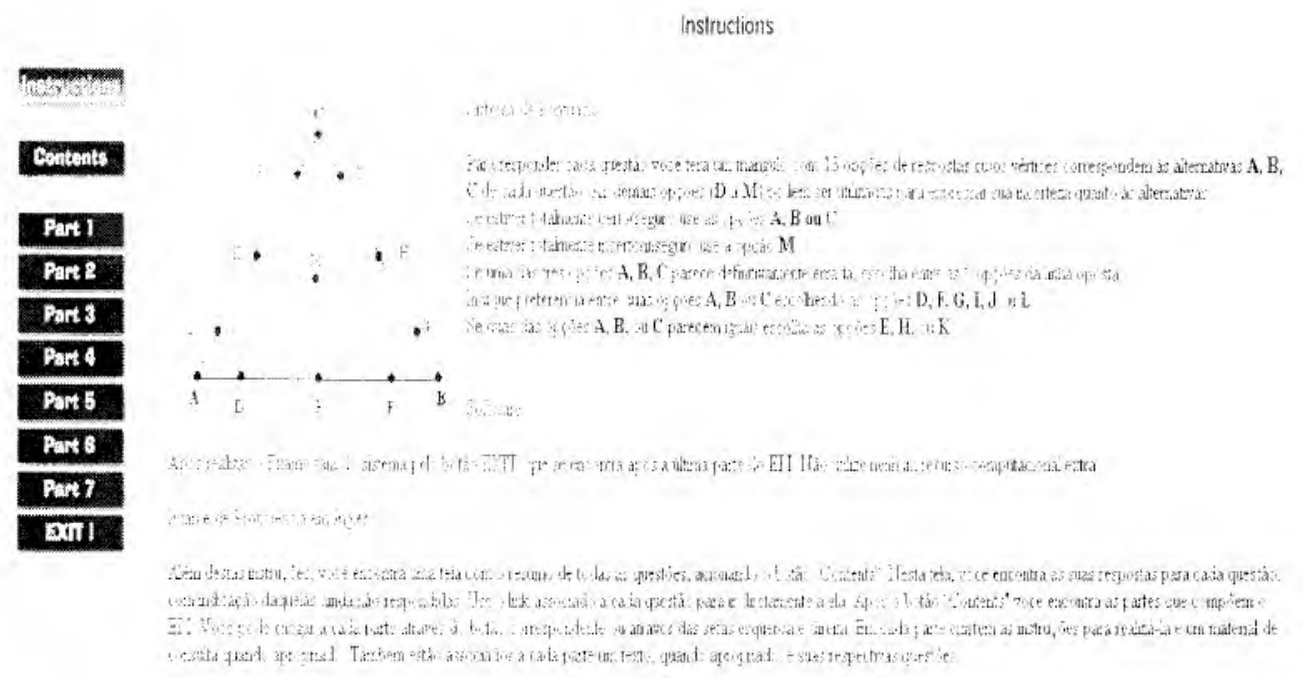

Fignar T.9: Interfiere de instrucoes do EPI

\subsection{Implementação}

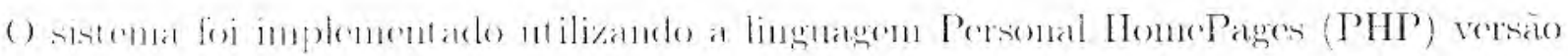

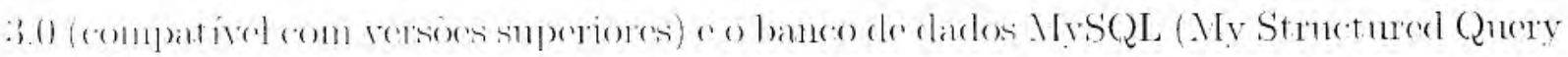

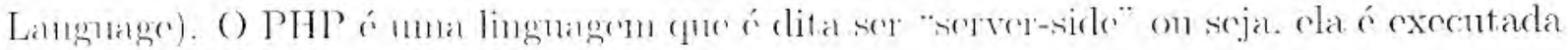

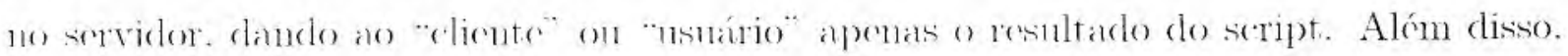

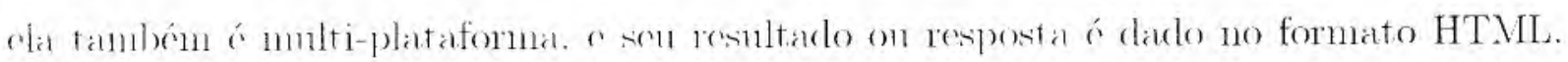
() PHP foi criaklo durante o ontono de 1994 por Rasmus Lerelorf. A razão da escolha da lingragem PHP se deve hasicamente ao fato de o cune porke ser feito por algum programa

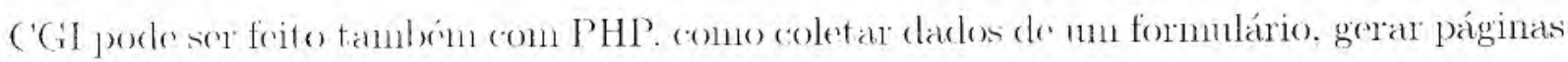

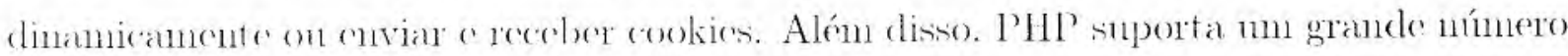

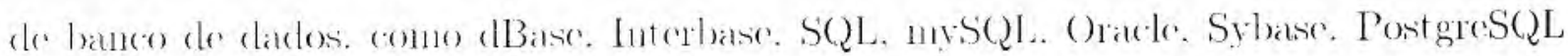

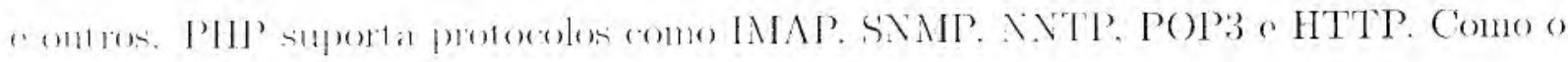

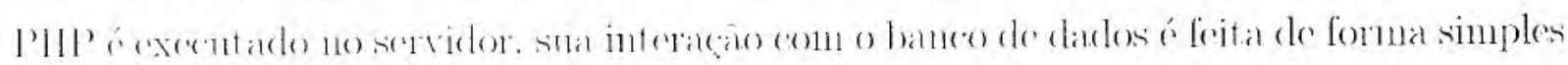

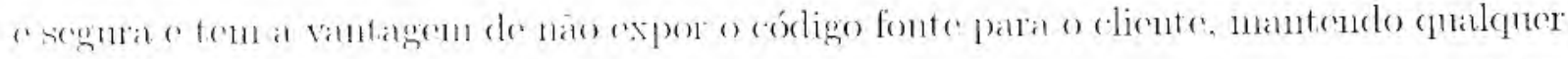

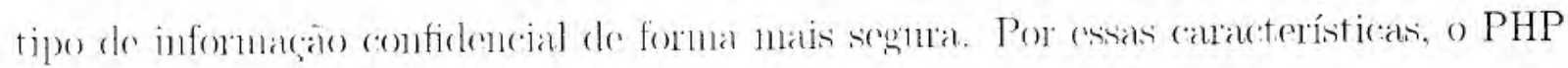



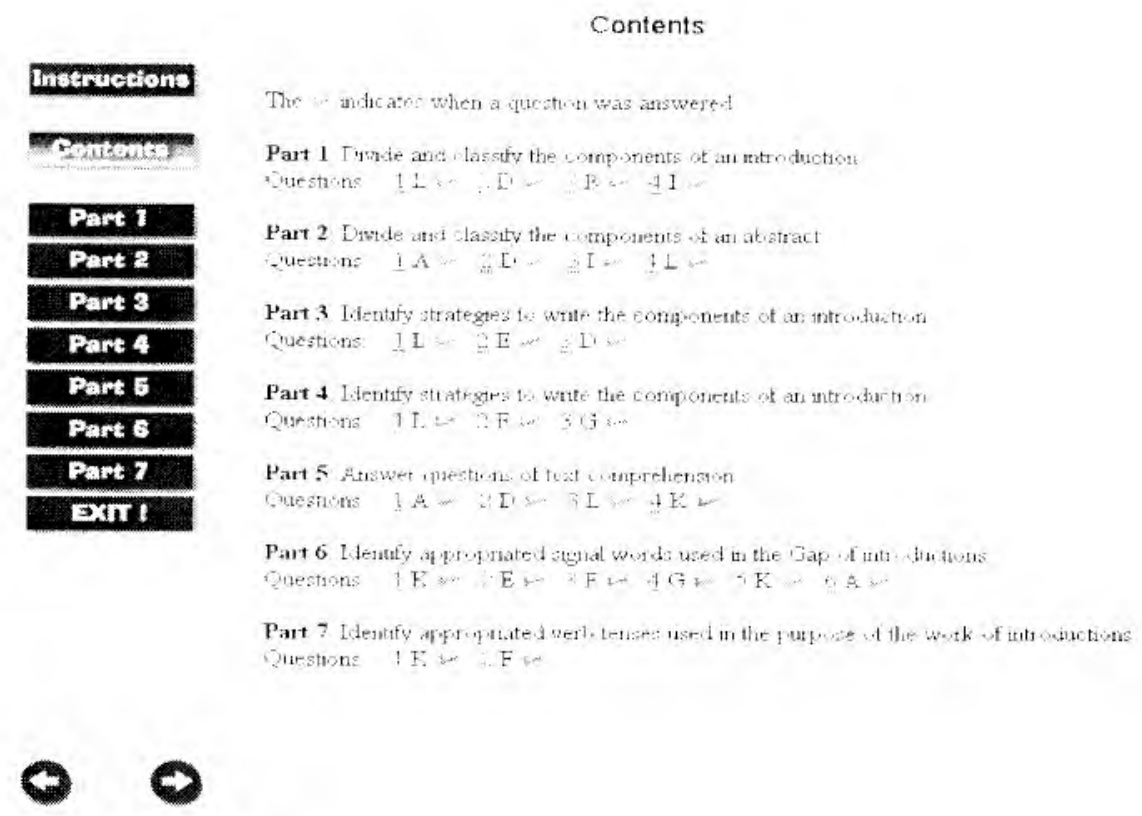

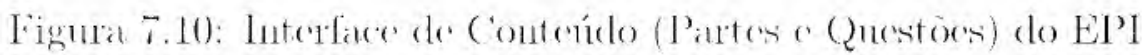

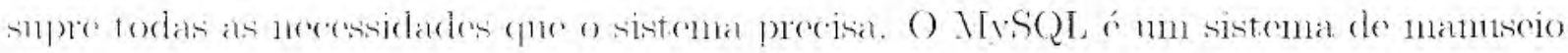

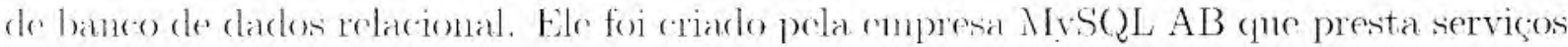
pala o mannseio de banco de dados namedo MyS(2L. () sistema de banco de dados ainda

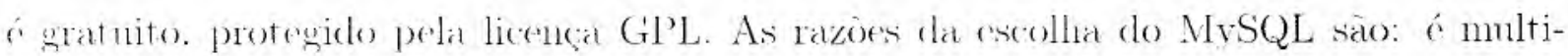

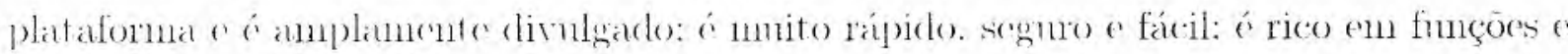

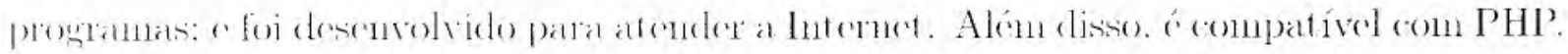

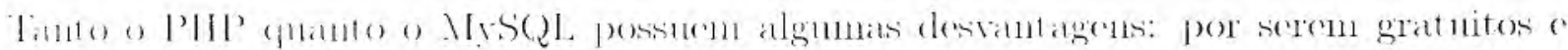

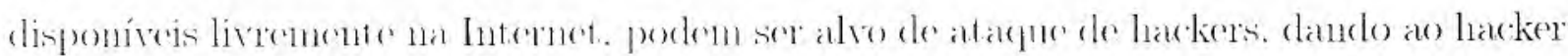

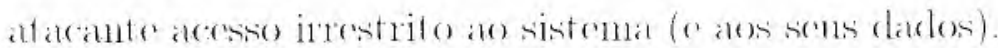




\section{Divide and classify the components of an introduction}

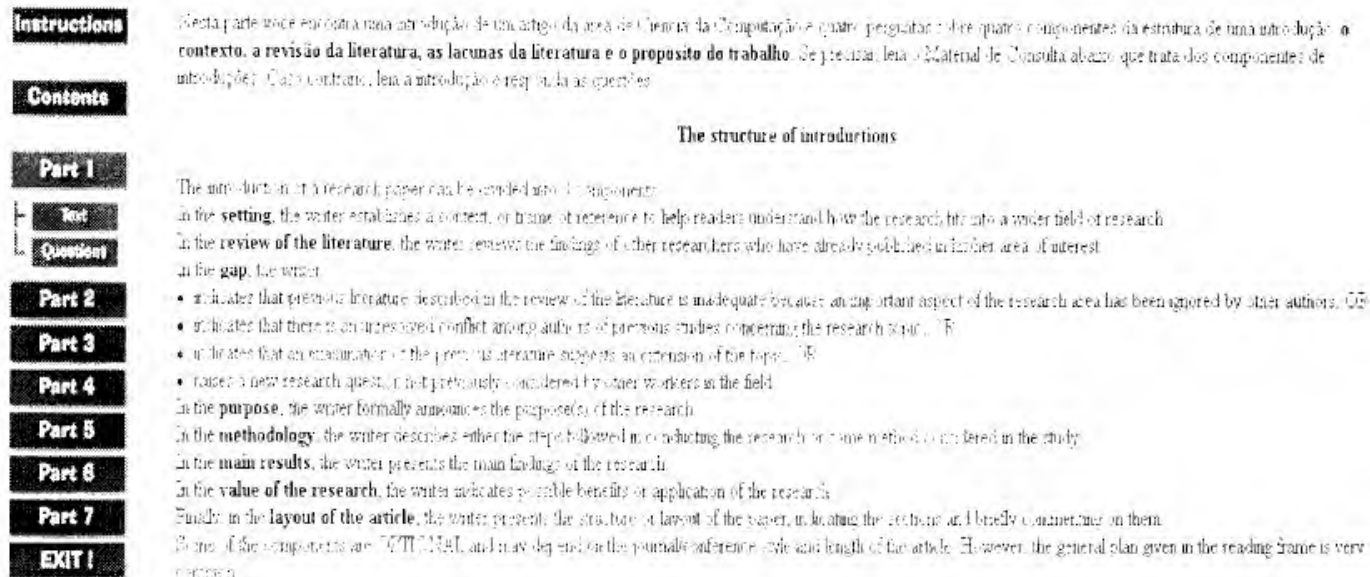

Figura 7.11: Interface de mua parte do EPI

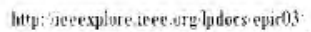

Cuniputer Graphirs Latemational 2001, Hong Kong, July 362001

A HYBRD APPROACH TO THE RECOVERY OF DEFURYAABLE SLPEROUIDEJC MODELS FROM 3D DATA

Inteructiont

Jantes Sinutt atud Tuby Howard

Contents

Par:

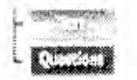

Pant 2

Part 3

Part 4

Part 5

Part 6

Rast 7

ETI!

Intradurtion

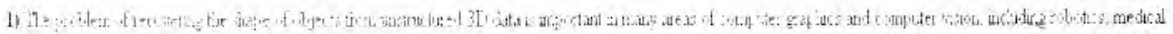

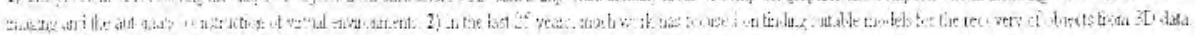

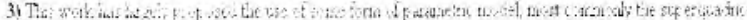

(a)

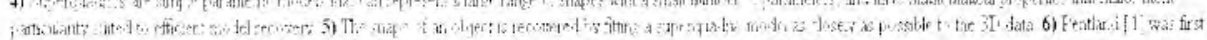

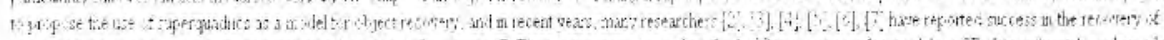

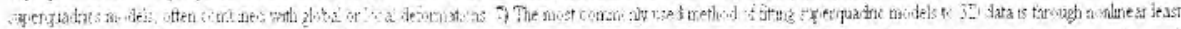

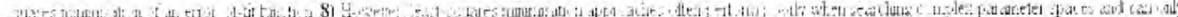

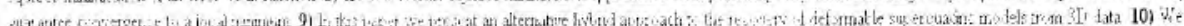
g.

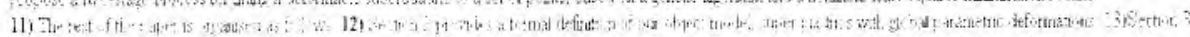

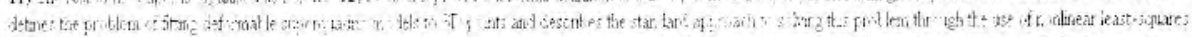

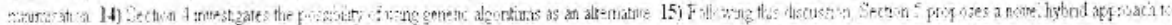

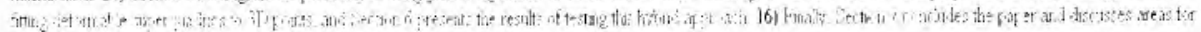
Live: rest:

\section{0}

Figma 7.12: Interlane gue apresenta of texto do man parte do EPI 
Part 1 : Question 1

Which sentence(s) in the intr. luction presented here correspond(s) to the settung?

\section{Instructions}

\section{Pontento}

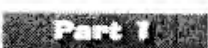

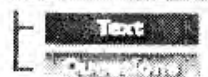

Pant 2

Pare 3

Part 4

Part 5

Pant 8

Part 7

3XITI

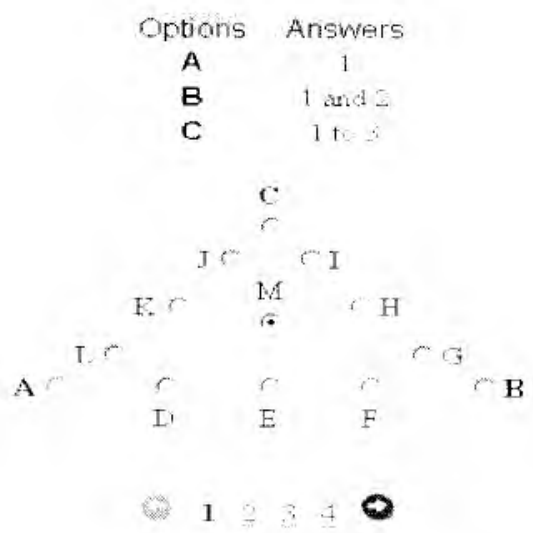

$\odot$

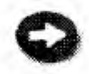

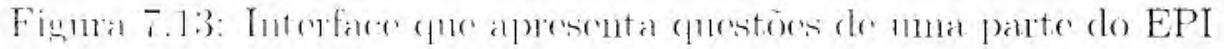

Identify stralegles to write the components of an introduction

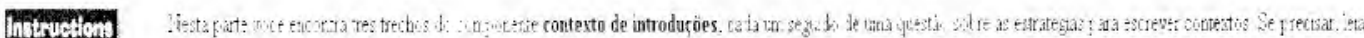

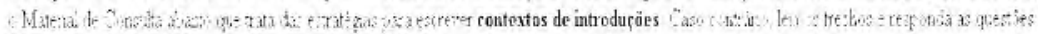

\begin{tabular}{|c|c|}
\hline Contents & Strategies to Setting \\
\hline Pant 1 & 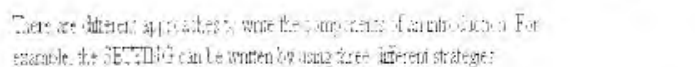 \\
\hline Part? & \\
\hline Pots & 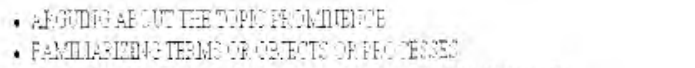 \\
\hline 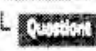 & 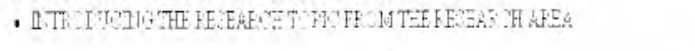 \\
\hline Part 4 & 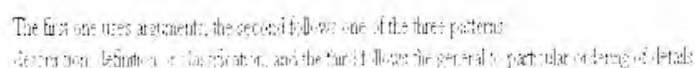 \\
\hline Pat 5 & \\
\hline Part 8 & \\
\hline Pant 7 & \\
\hline EXI! & \\
\hline
\end{tabular}

\section{0}

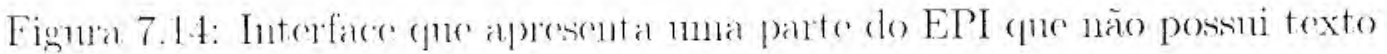




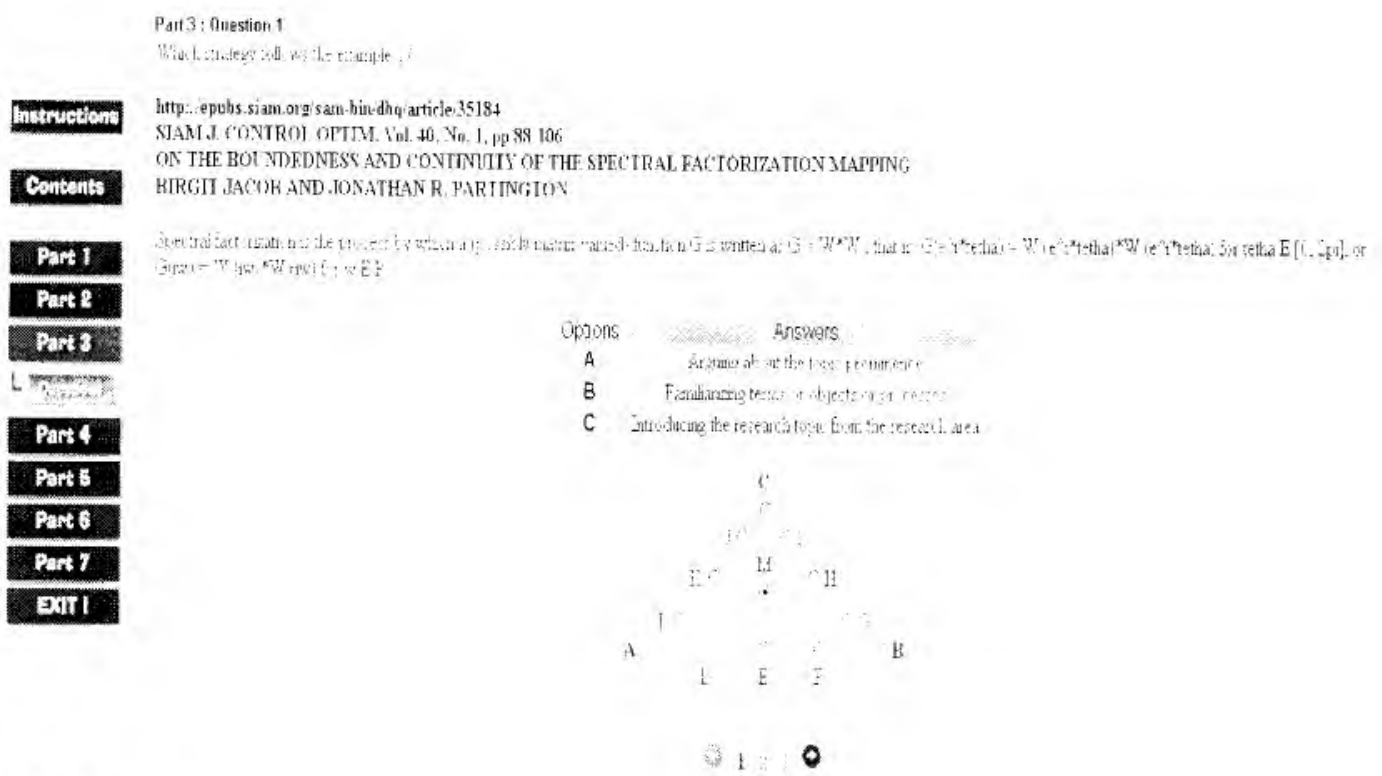

00

Figman 7.15: Interface (pur apresenta questoes de nund parte do EPI associadas a trechos de textos

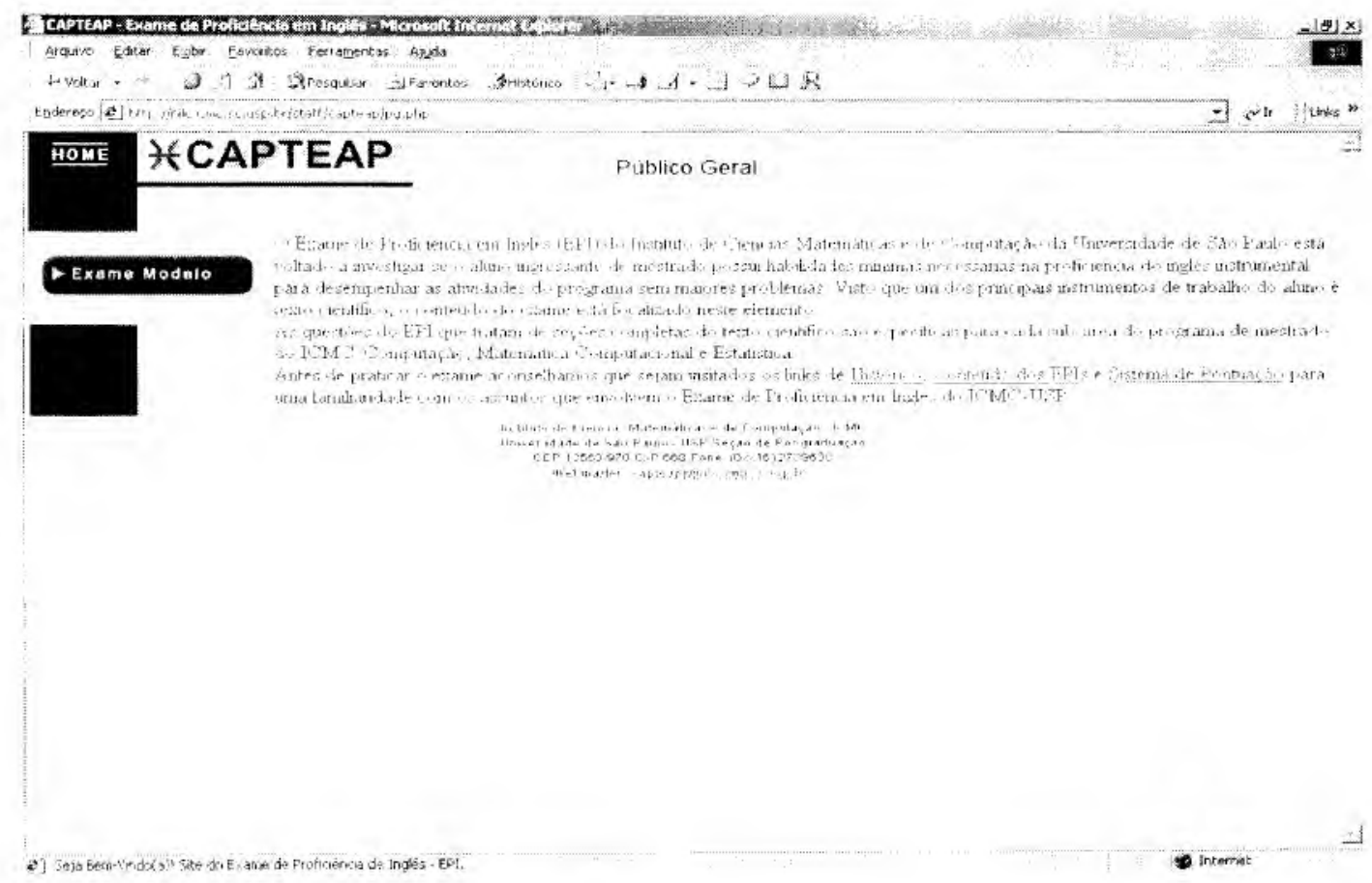

Figura T.16: Tutreface do público geral 


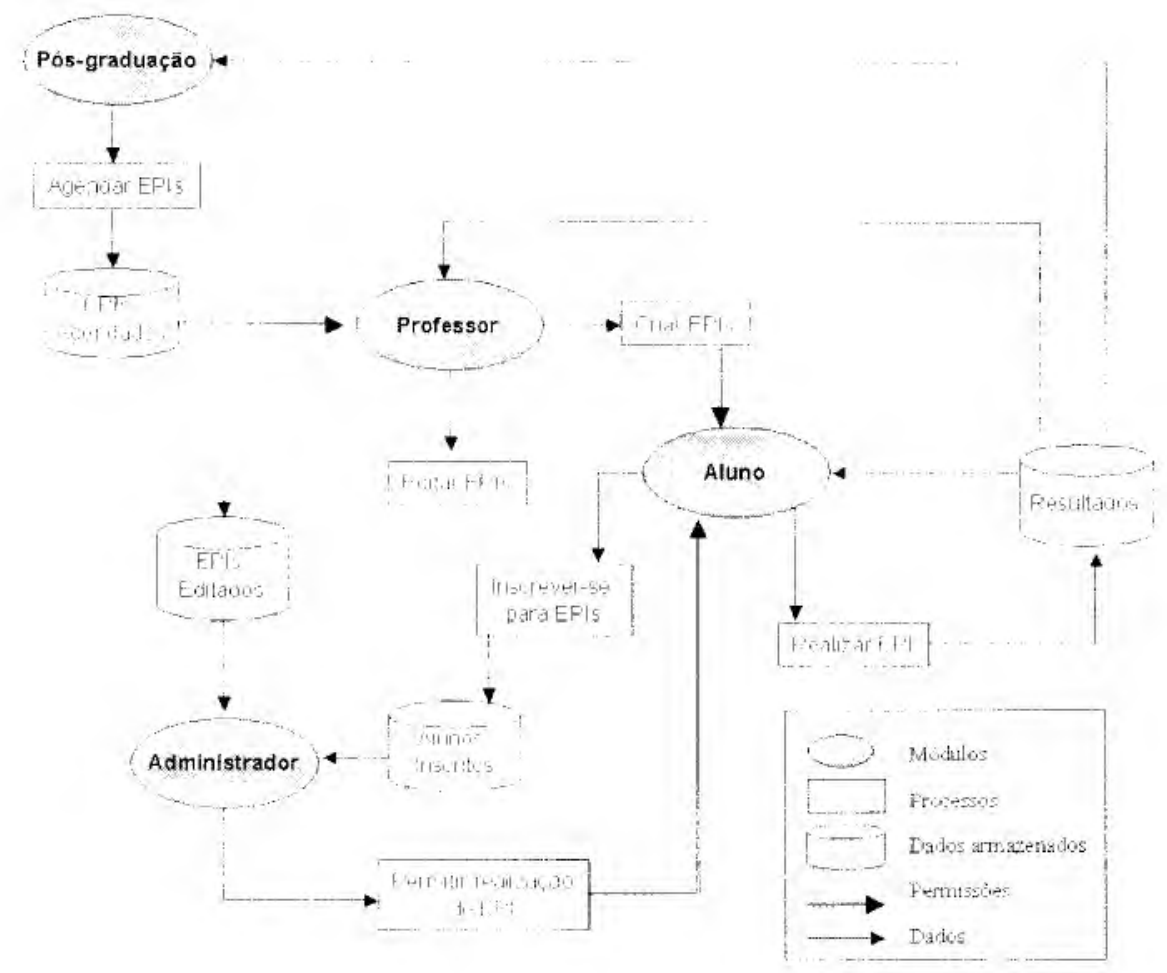

Fignua 7.17: Diagranat de blocos geral dos principaris módnlos do EPI 


\section{Capítulo 8}

\section{Conclusão}

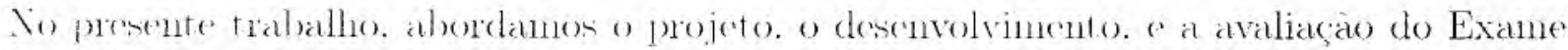
Antomatizado de P'roficione ia en Ingles para o progranta de nestrado do ICMC-LSP. A

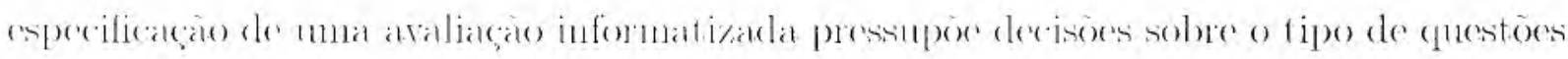

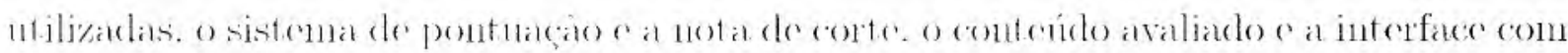

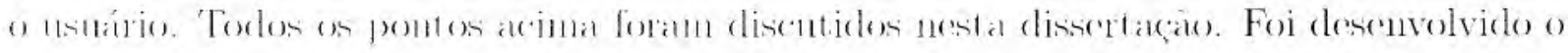

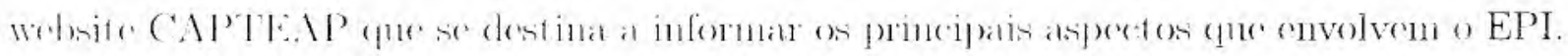

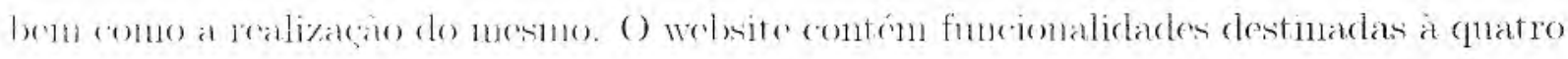

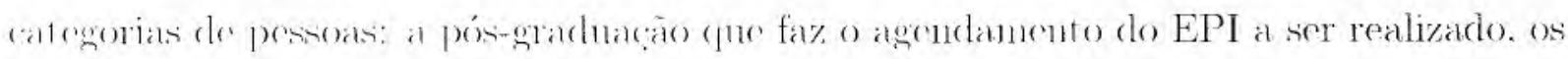

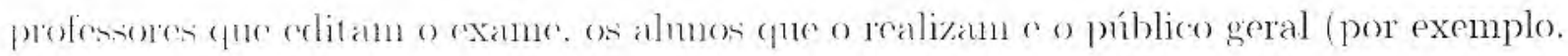
futures ingressantes no progranua do pós) que pode ter um comliecimento prévio a respeito (lo El'l ntilizado no IC'MC'-LSI'.

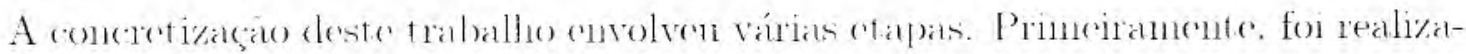

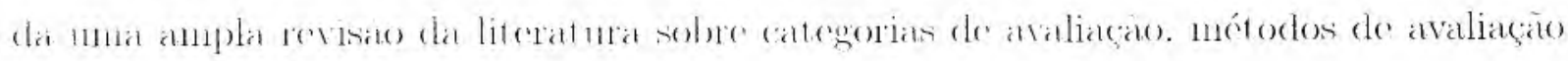

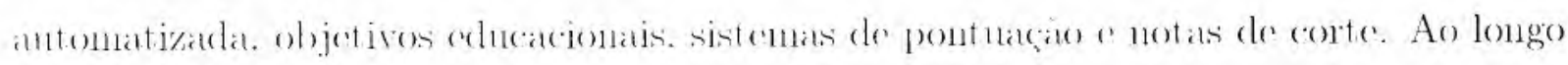

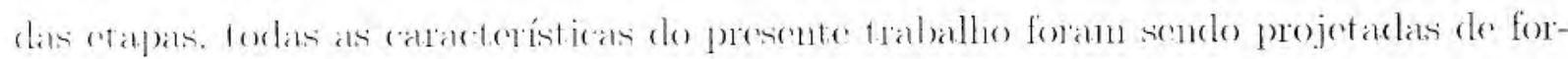

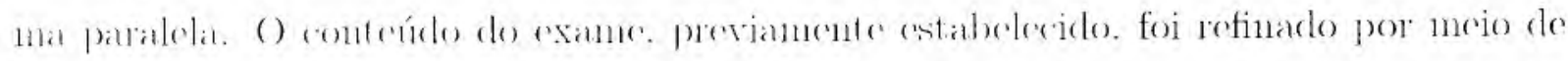

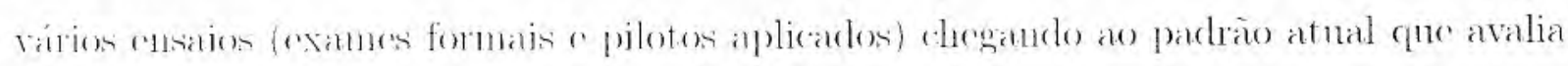

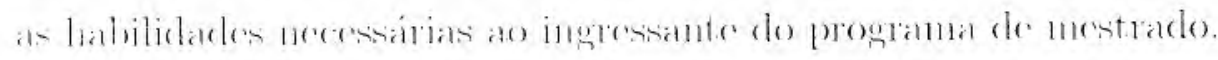

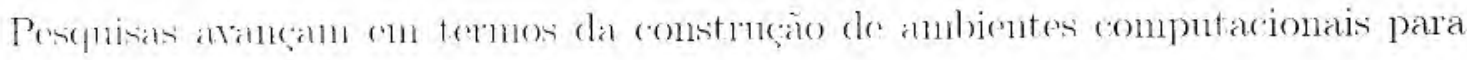
anxiliar a construcão do coulherincuto. portanto a avaliaço informatizada necessita an- 


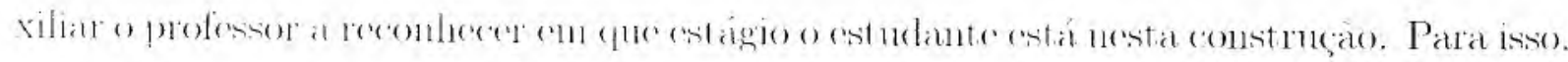

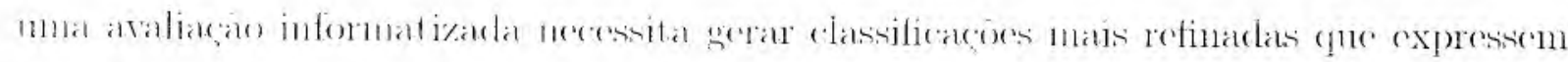

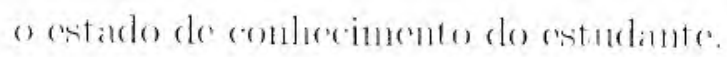

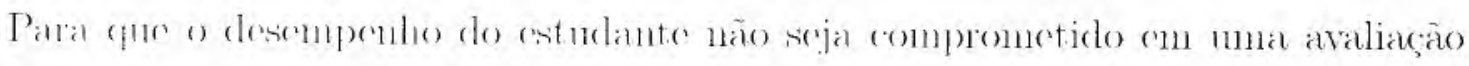

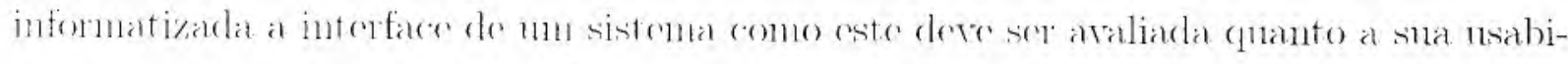

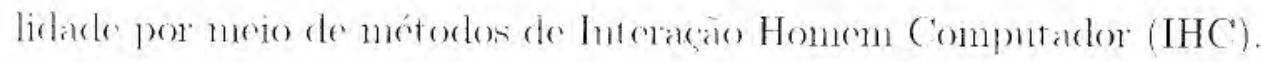

Apesar de utilizar um ermirio esperifieo (Fxane de proficiencia en Ingles) o sistemat criado nester trabalho de mestrado pode ser ntilizado para gerar avaliacoes em qualquer área. Desta forma. de contribuin com mua proposta do mu método alternativo de ava-

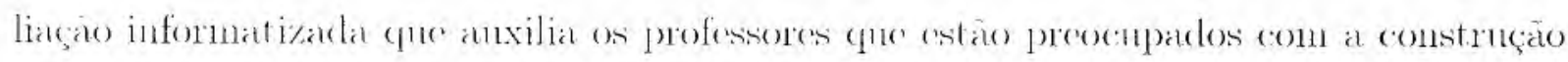

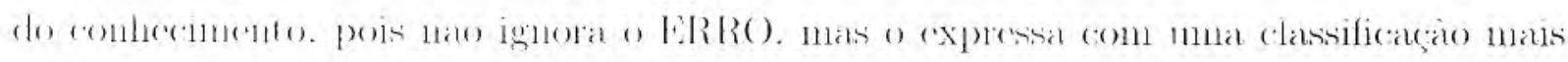

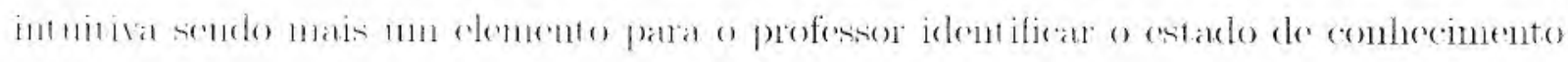

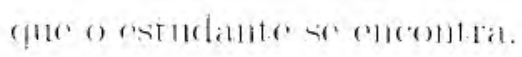

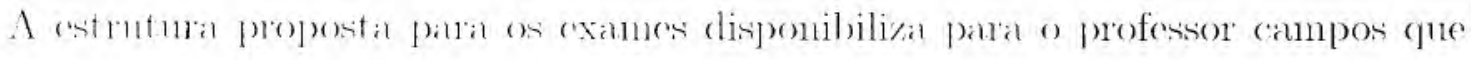

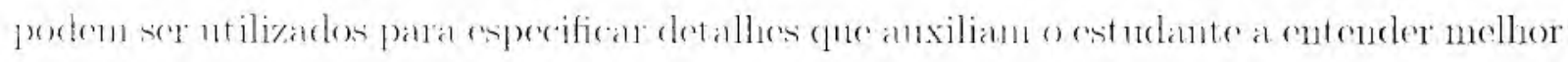

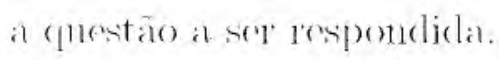

() processo de desemvolvimento do trabalho facilitom o entendimento quanto ao

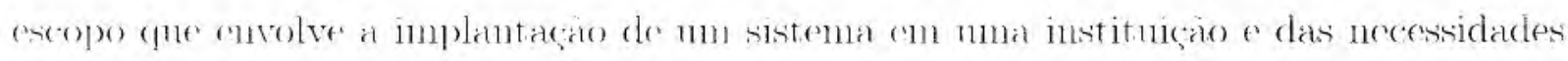

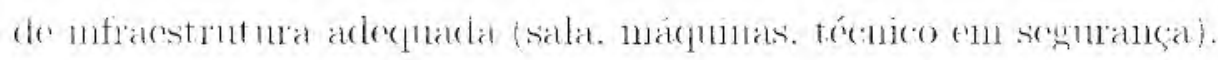

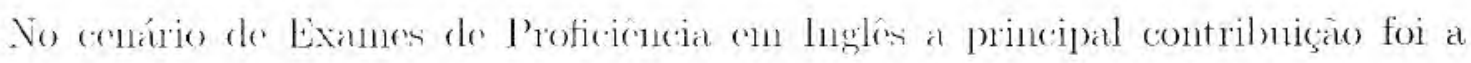

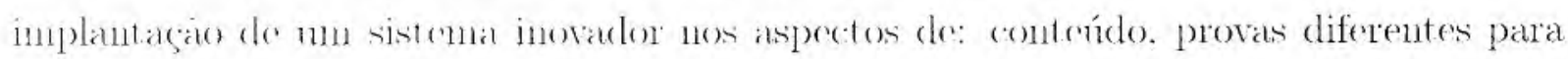

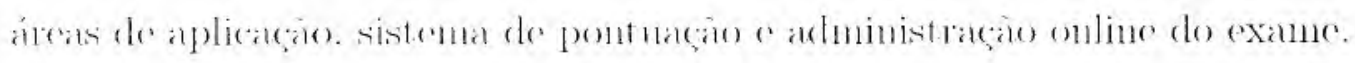

\subsection{Trabalhos Futuros}

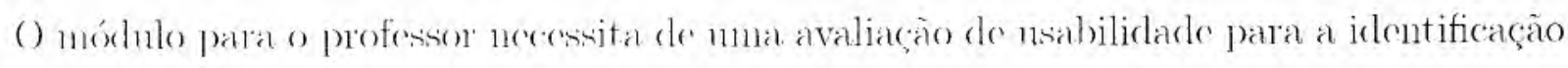
de problemas e sugestoes do fincionaliclades a serem acrescentadas. Existe a necessidade

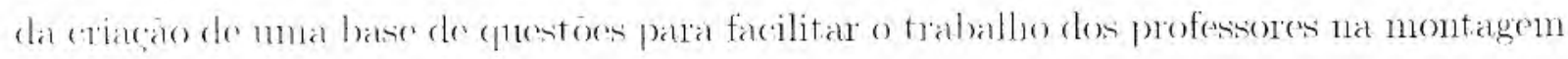
(1) El'l.

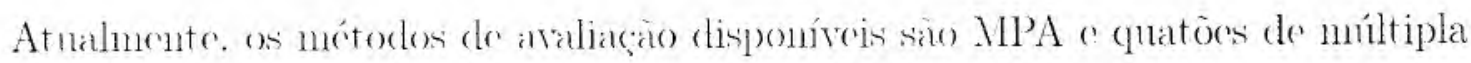

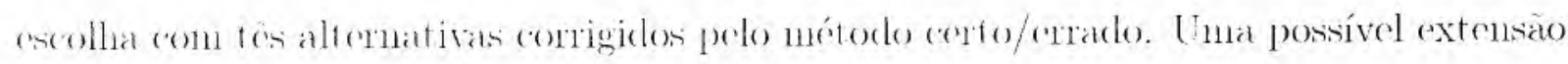




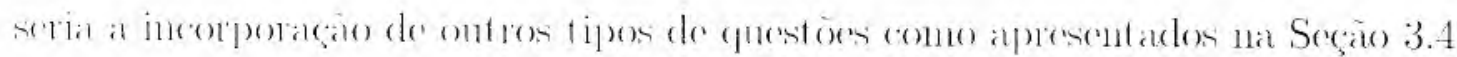

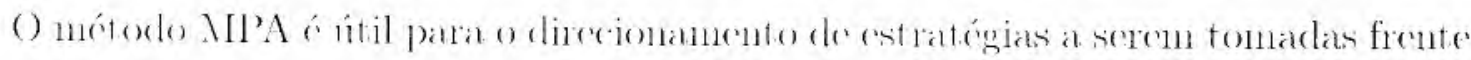

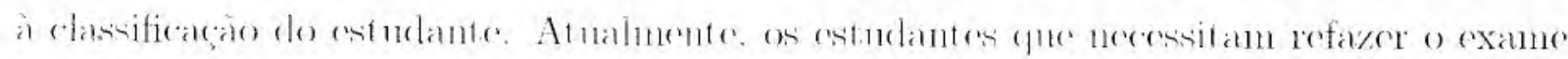

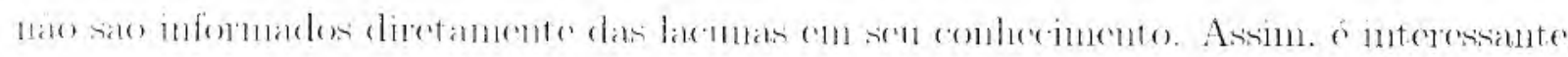

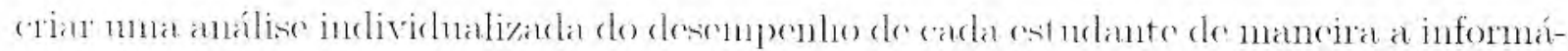

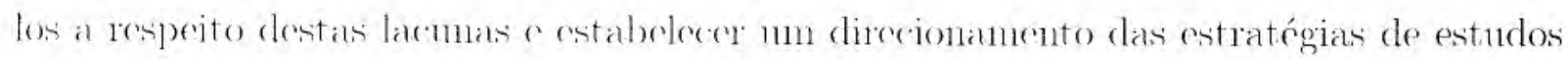
sols a forma de run tutorial.

Cin outro traballuo futuro seria reavalian a reestrutumar os aspectos de segurança

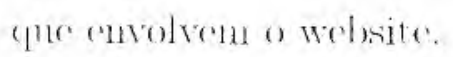




\section{Referências Bibliográficas}

Alessi. S. II d Trollip. S. R. (1991). Computer-Bused Instruetion-Methods and DeveLommunt - Znd Edition. Prentice Hall. Lne.. Englewood ('liffs. New Jersey 07632.

Aluisuo. S. M. \& Crantenlerin. E. R. (1997). Towareds the application of systente functional linguistics in writing tools. In Prosedimgs of International Confromer on Computers and their Applications. pages 181 185. Arizona. CSA.

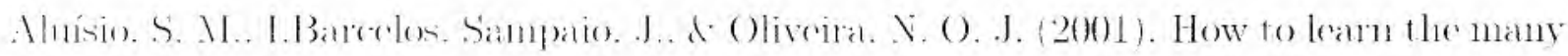

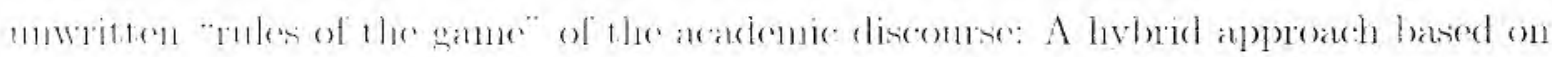

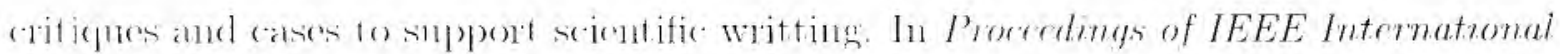

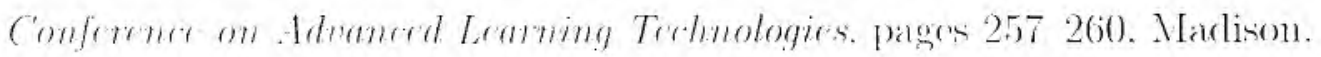

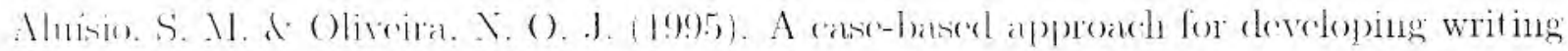

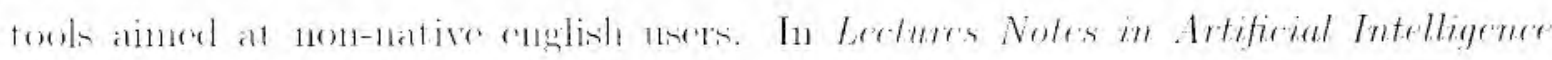
11101) patges 12! 13:2.

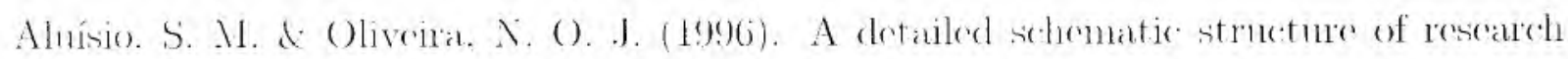
patper introductions: An application in support-writing tooks. In Revista de la So-

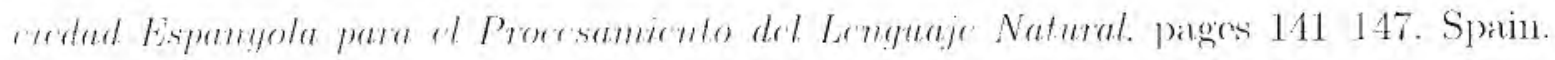

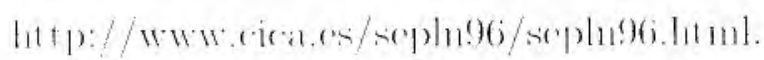

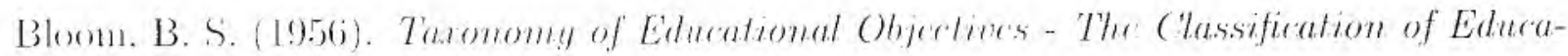

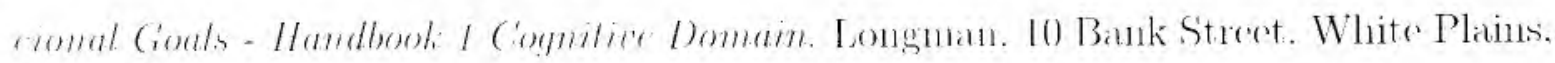

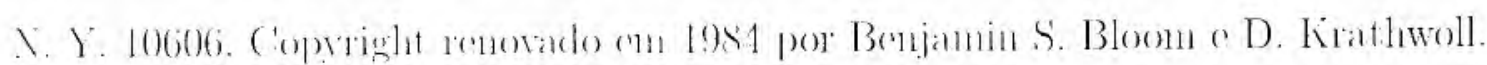

Brown. S.. Rare. P.. d Bull. J. (1999). Computer-Assisted Asscessment in Higher Edurafion. SEDA - Statf and Edheational Development Series. 


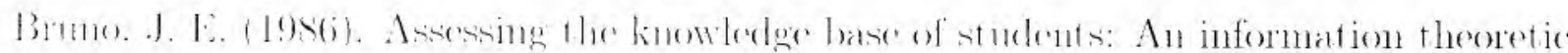

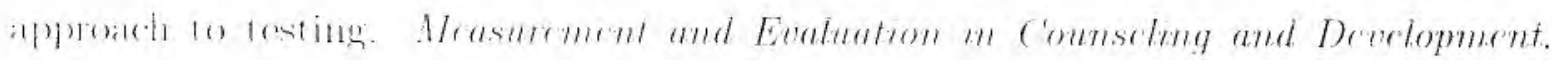
patges 117130 .

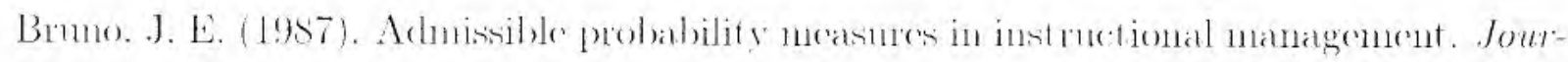

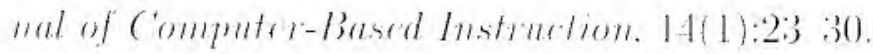

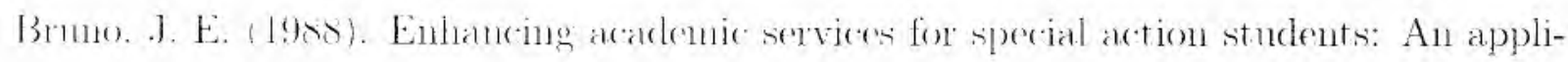
ation of information referemeed 1esting. Measurement. and Enaluatom in Counselang and De owelopment. patgens 515.

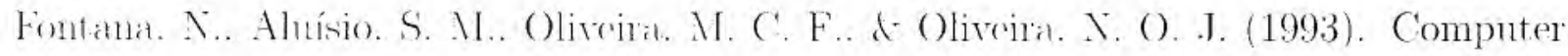
assisted writing - applications to cuglish as a foreign language. English for Spectific Putpost's. (6(2):145 161 .

Klinger. A. (1997). Experinental valiclation of Garming accomplishment. Procepdings Frontifis in Eduration. page 6.

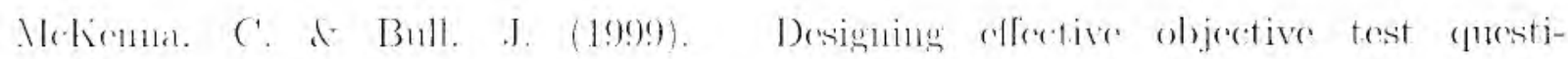
(nis: an introductory workshop). In Procerdings of the Thind Annual Computre Assisted Assessment Comfermer pages 25:3 255. Lomghboromgh. England.

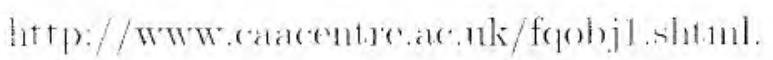

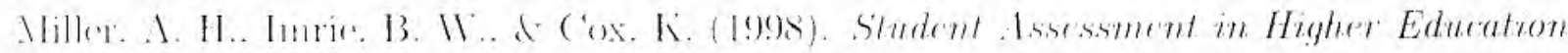

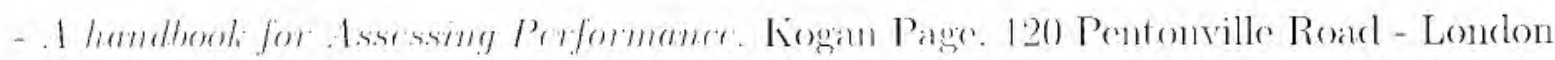
(1) 9.1

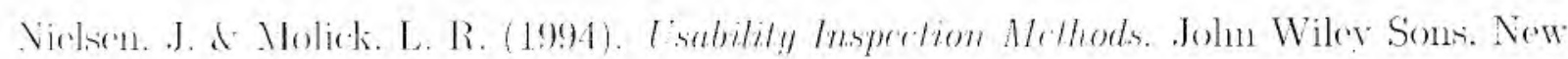
roik.

()lora. J. Ponsocta. V.. \& Prieto. G. (1999). Trists Informatizados Fundamentos y Aplica-

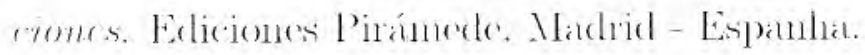

()wen. R. (1975). A baysesian secplancial procedure for quantal response in the context of arlaptive montal testing. Jommal of Americon Statistiral Association page 70 . 


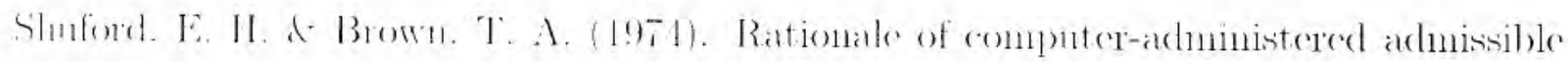

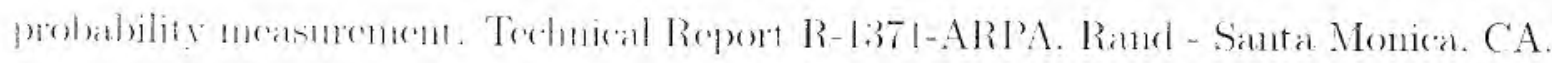

Sibler: W. L. (1974). A prototype computer prograu for interactive computer-

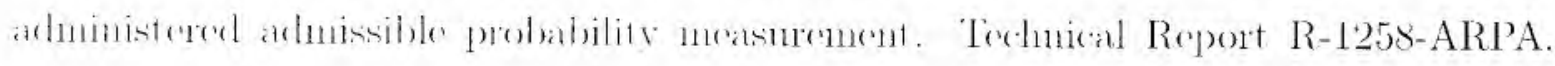
Rimul- Sinnta Mlonica. ('A.

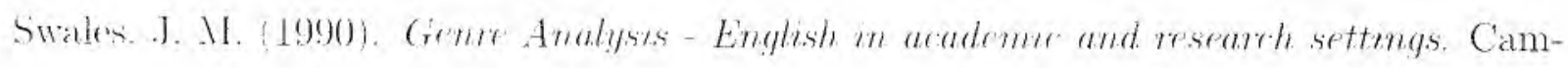
inielge Lniversely Press. Cambridger. 


\section{Apêndice A}

\section{Exame formal realizado em Abril de}

\section{8}

Instituto de Ciencias Matemáticas e de Computação

Departamento de Ciencias de Computação e Estatística

Progranna de Pós-graduação do Instituto de Ciências Matemáticas e de Computação

English Examination - 29/04/98

Profa. Sandra Maria Alúsio

(Part 1) The inteoluetion of the researed articte (a) be divided into 8 parts. or stapes. Lis Stage I. the miter establishes a contexi. or frane of reference to help readers muderstand how the researele fits into a wider field of study. In Stage II. the writer

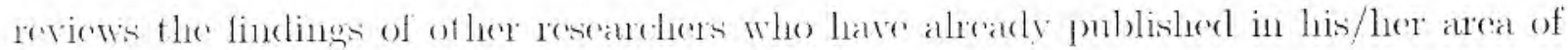
interest. After Stage I and II. the writer uses the final part of the introduetion to focms

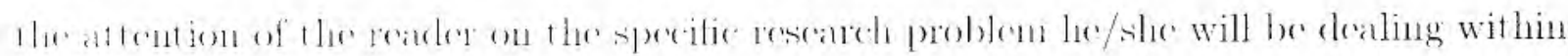

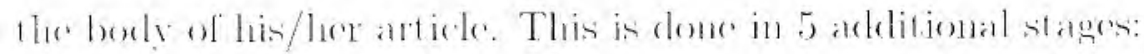

1. In Stage III. yon may indieate that the previons literature described in Stage II is inalefpate becantise an impontant aspeet of the researeh area has been ignored

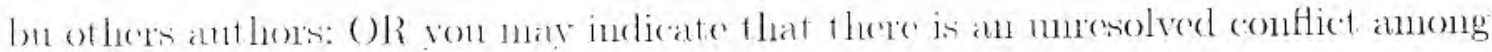

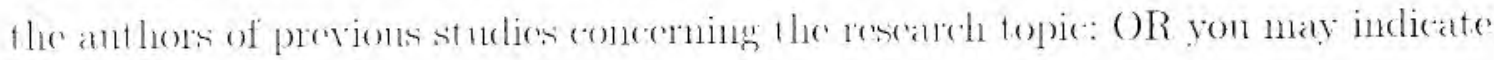
Hat an examination of the previons literature siggests an extension of the topice, 


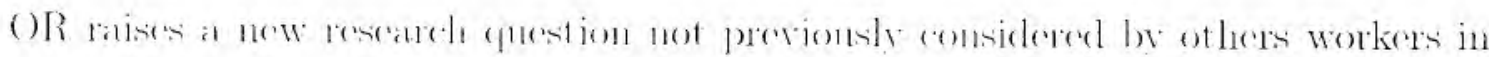
ron! tickl.

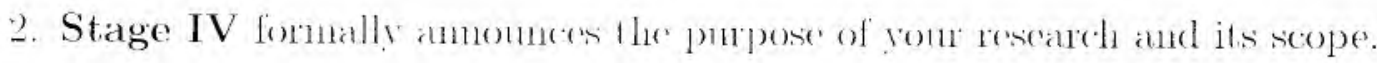

3. Stage V. the nethorklogr: dereribes the steps you followed in conducting vour sturly:

1. In Stage VI. Ile pesults. wou present the findings of vour studs and briefly comment (1) themit

5. Stage VII indicates possible bencfits on application of your work.

Finally: in Stage VIII. you present the struetume/layont of your article. indicating the sections and briefle commenting on theme

bome of these stages are optional or parts of them are optional. It may depend

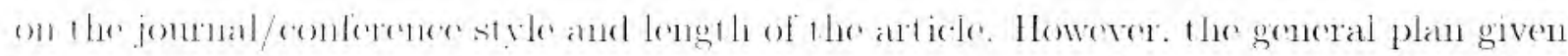
above in very (onthenen.

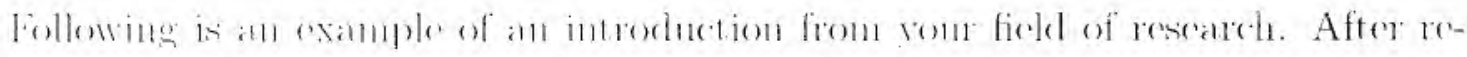

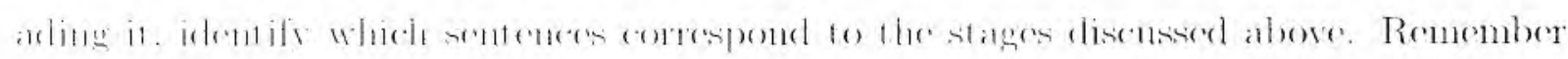

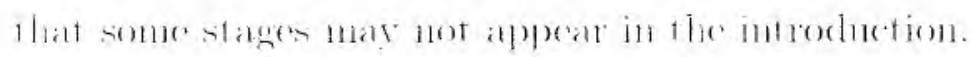

Austral. J. Statist. 39(2), 1997, 129-148

SOME SECOND ORDER ASYMPTOTICS IN EXPONENTIAL FAMILY NON-LINEAR REGRESSION MODELS (A GEOMETRIC APPRO$\mathrm{ACH})$

BO-CHENG WEI AND HONG-TU ZHU

Southeast University, Nanjing

Summary

The eliferencial geometric framersork of Amari (1982a: 1985) is applied to the

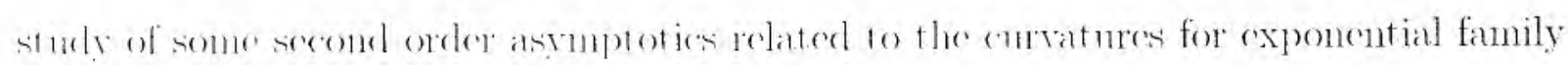

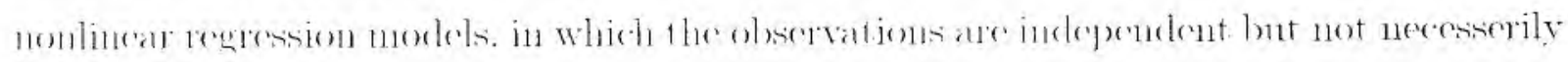




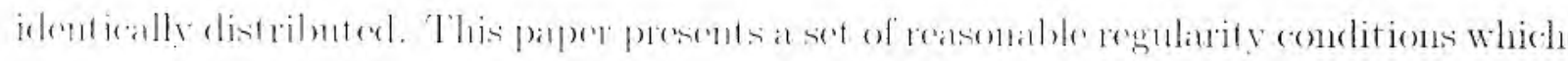

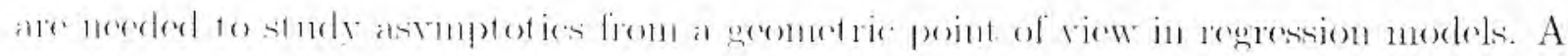

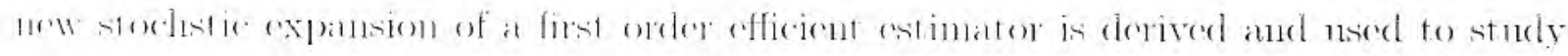
sereal astmptotic problems redated to Fishere information in temes of anvatumes. The bias and the covarianee of the first oretere efficient estimator are also calculated aceording to Hor (x) antsion.

Lify words: Cinvatme: axponential fantly noulinear regression models: first order rflicient estinanor: infornation loss: regularity conditions: stochastic expansion.

\section{Introduction}

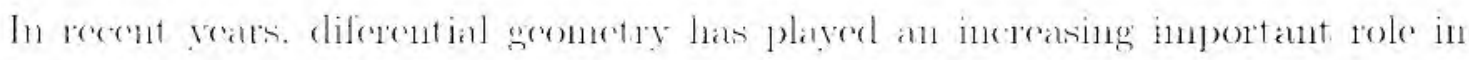

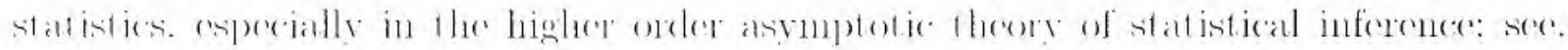

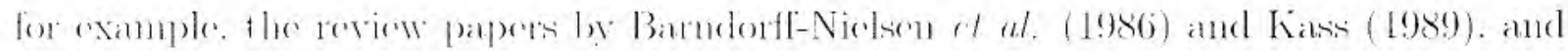
looks he Anari (1985) and Murra d Rice (19993). Elion (1975) and Anari (1982a.b)

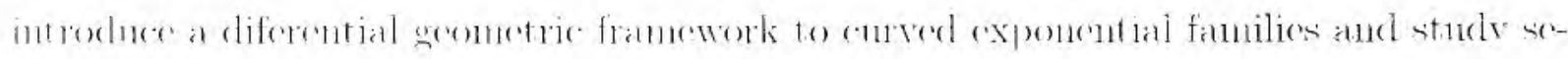

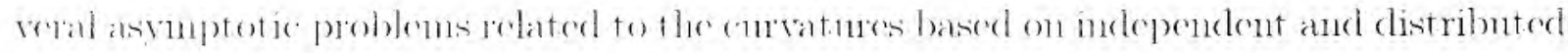

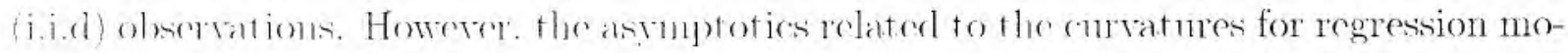

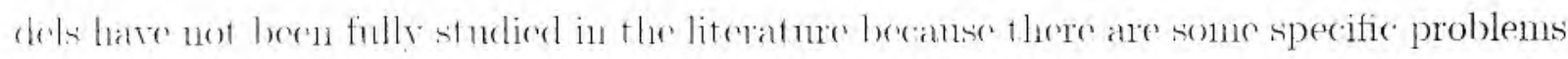

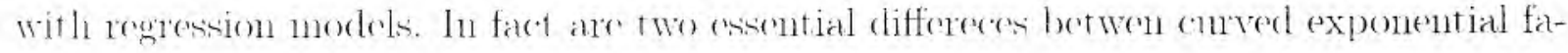
milies with i.ted observatons and regression nodels. Finst. The observations in regression

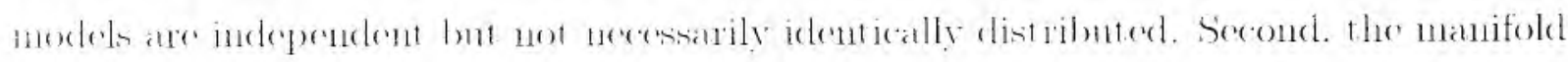

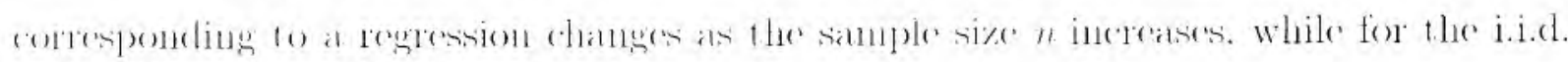
atsire studied.

1, Elron (1975) and Amari (19\$2a). the geometrie is actually based on one of the i.j.d. observations (Anari. 1985). Therefore the astmptotice in the i.i.d. case are

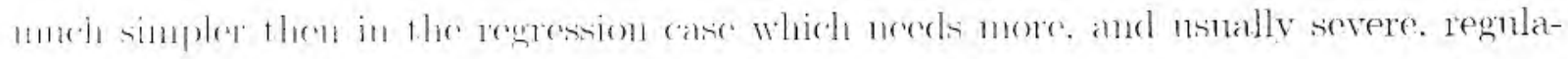
rity conclitions. In this papor. We apply the geonetrie gramework of Amari (1985) and temsor notation to study some socond oreler astmutetics for estimatord in exponential fanily nonlinear regression modeds. We present a set of reasomable and relatively simple

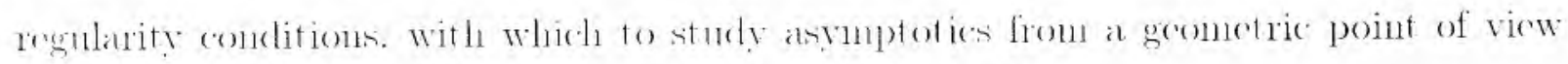




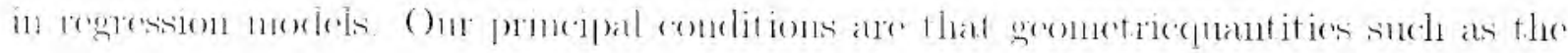

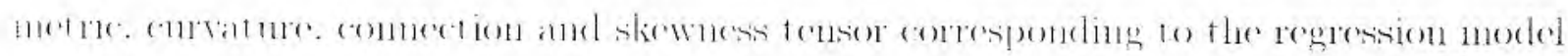

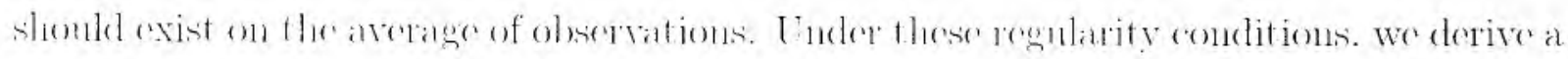

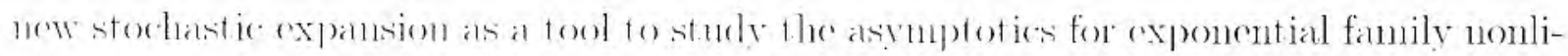

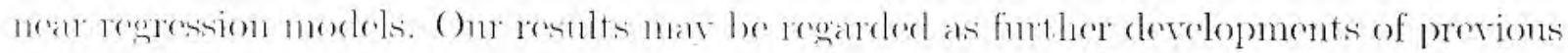
work by Efrom (1975). Efrom d Hinkler (1978). Amari (1982ah) and Woi (1991). Section 2 deseribes the geometric asperets of expouential fanily nonlinear regression models based on the geometric franework of Amari (1985). Section 3 derives a new stochastic expansion for first orelen eflecint estintators. Section 4 investigates the information loss and gives a limit theremenemested with the observed information and the expected information. In seretion 5. We calculate the bias and the covarianes of first onder efficient estimators. based

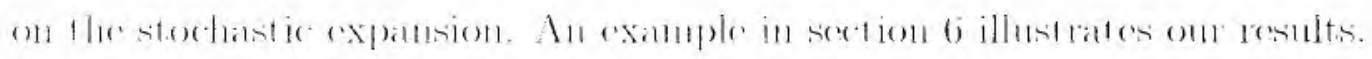

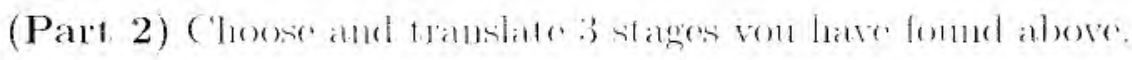

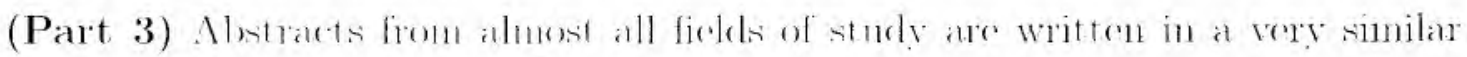
way: The types of infornation indedere and their ofder are very comsentional. The box that follows shows the typical infomation format of an alostract.

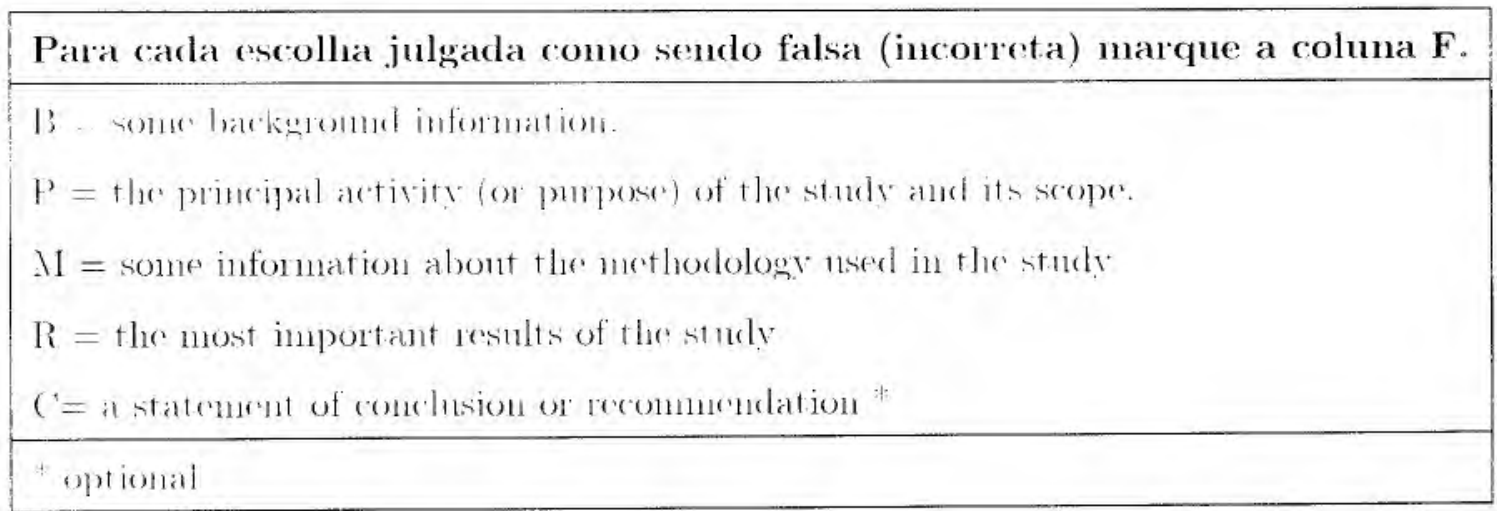

a) Write an abstrate in binglish for some work you have undertook dlumg vour mudergraduate or graduate programis.

()R

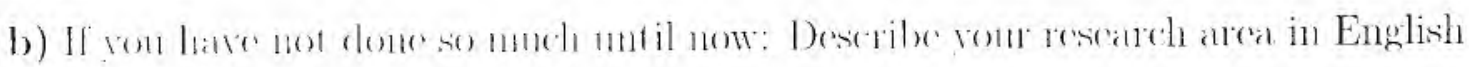
10 limes). 


\section{Apêndice B}

\section{Exame piloto realizado em Setembro de 1999}

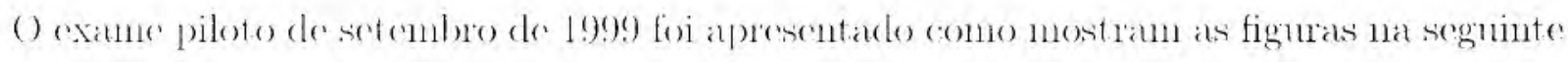

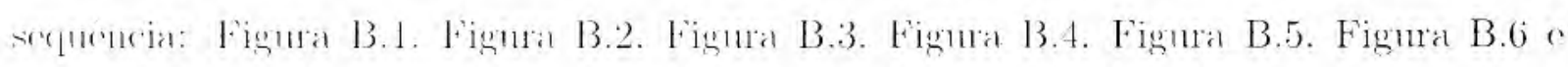
Fignad B. $\bar{i}$.

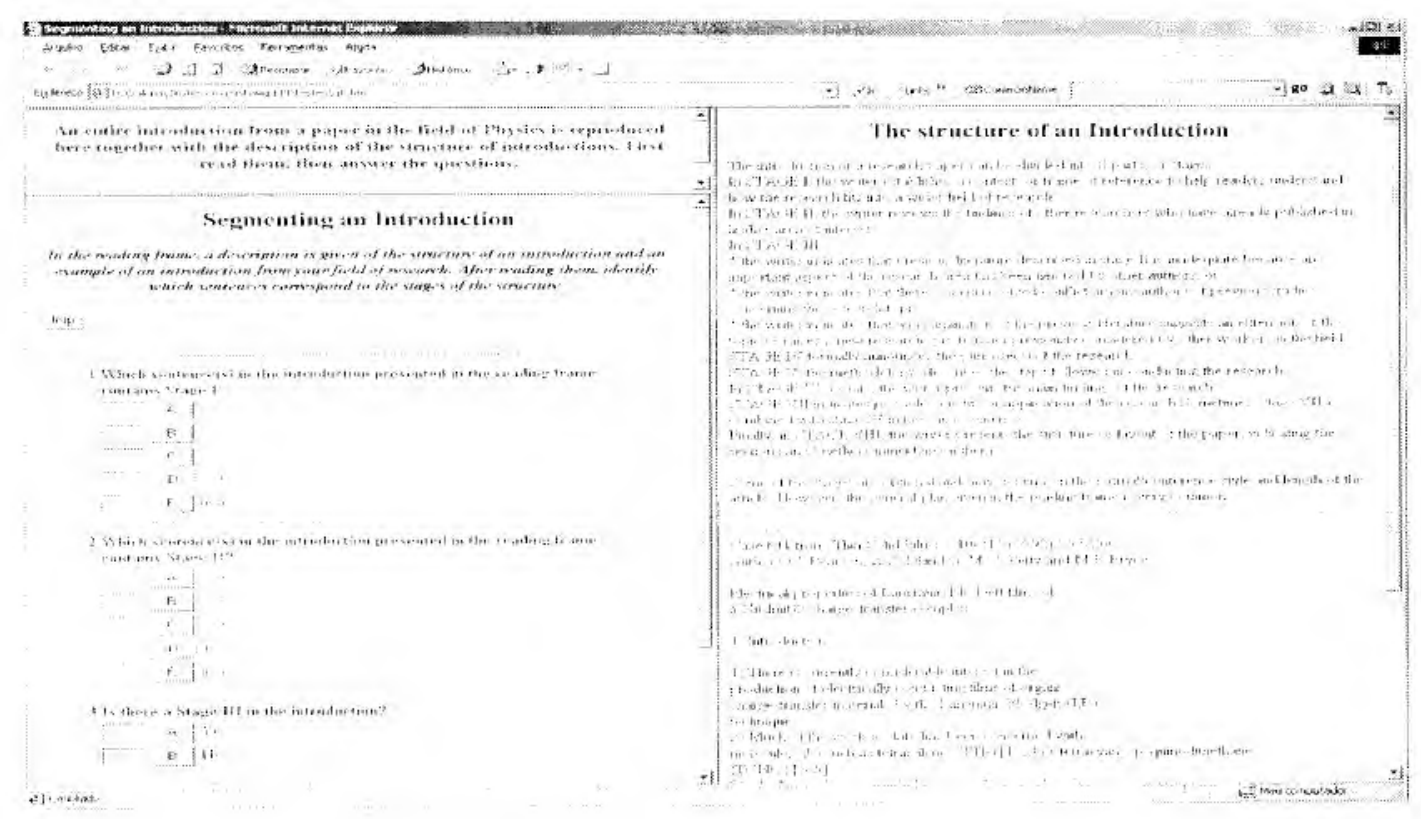

Figna B.1: Segunenting an Hetroduction 


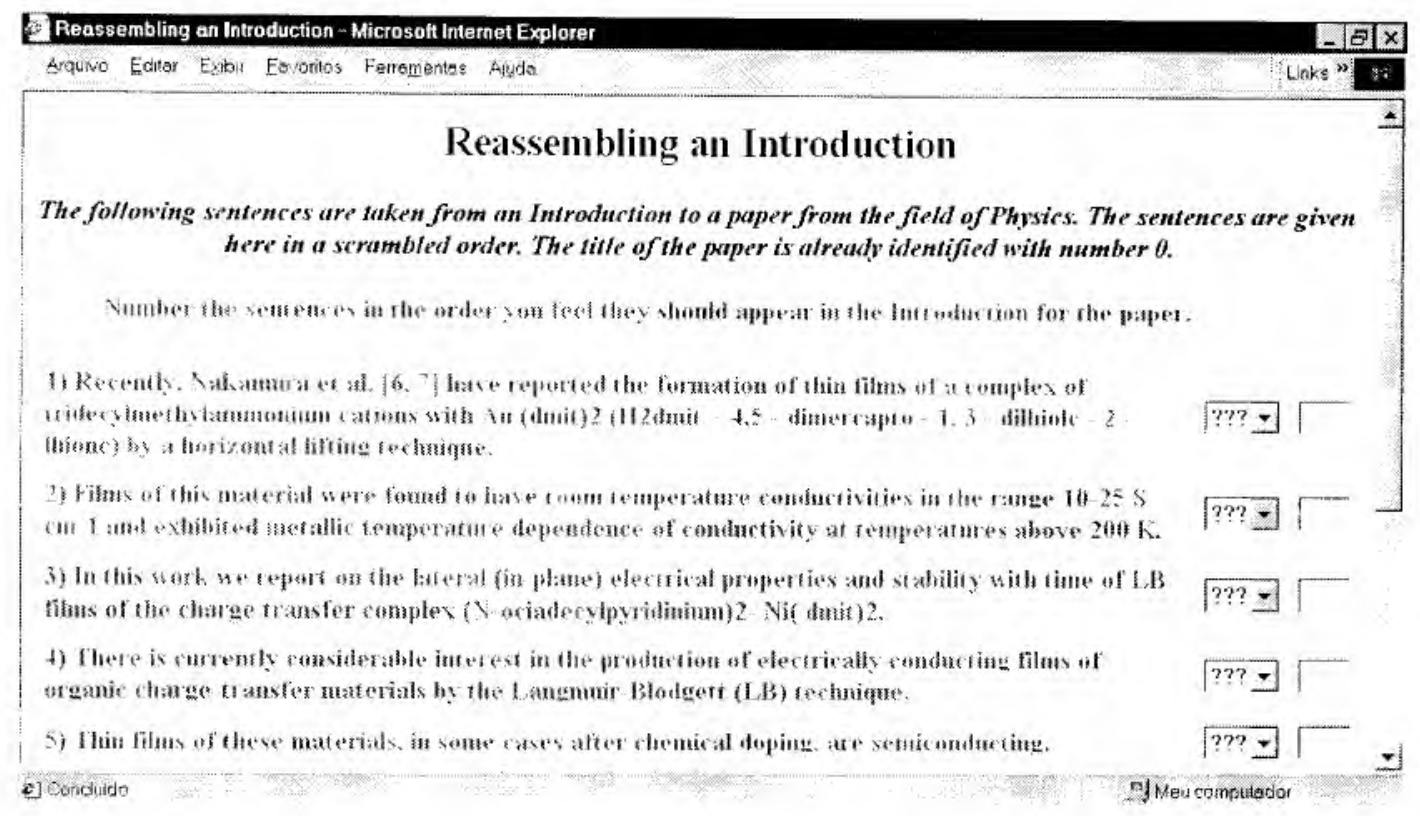

Figura B.2: Romsombling an Dutrodiction

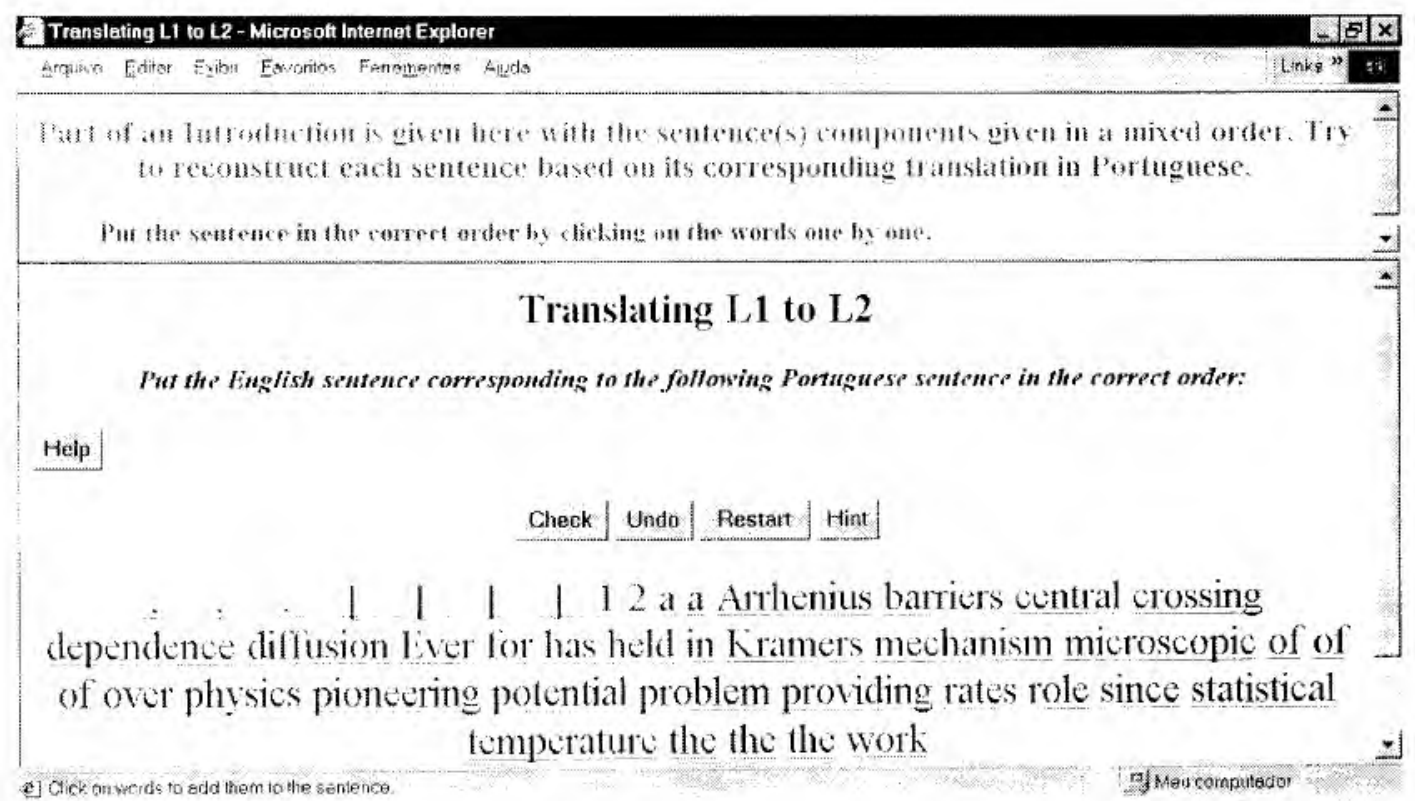

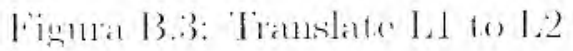




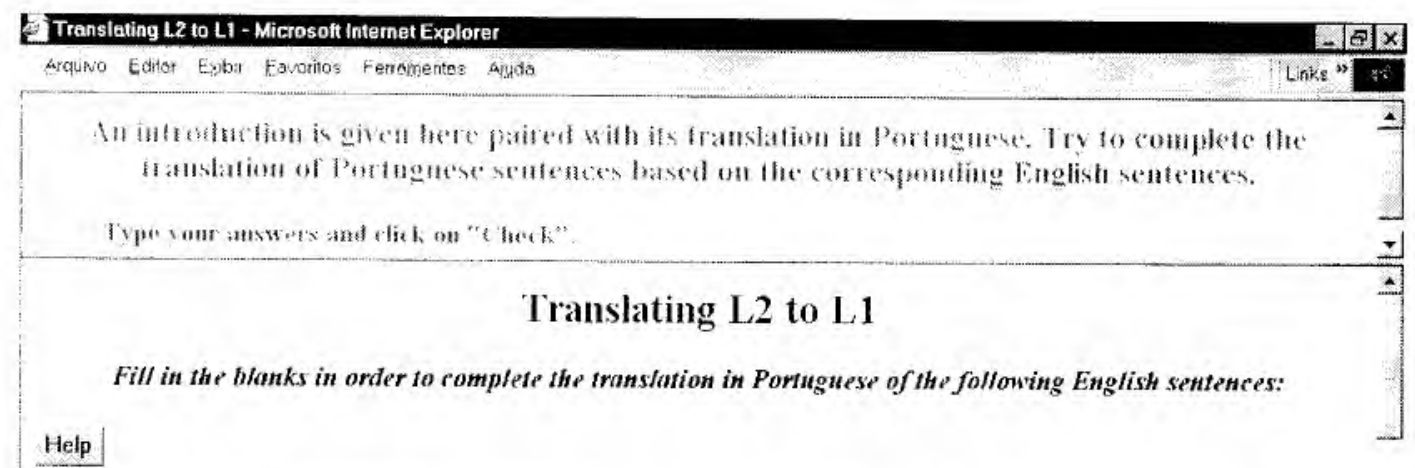

Help

There is currently considerable interest in the production of electrically conducting films of organic chargetransfer tmaterials by the Langmuir-Blodgett (LB) technique.

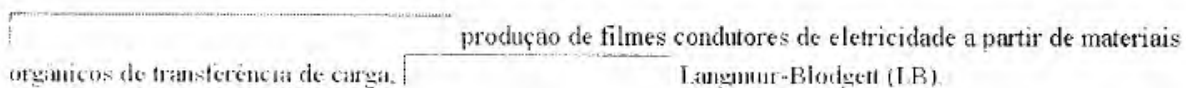

Much of the work to date has been concerned with molecules such as tetrazalene ( TTF) [1. 2] or tetracyano-p-

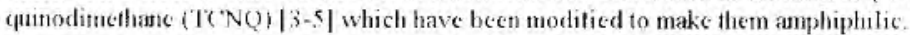




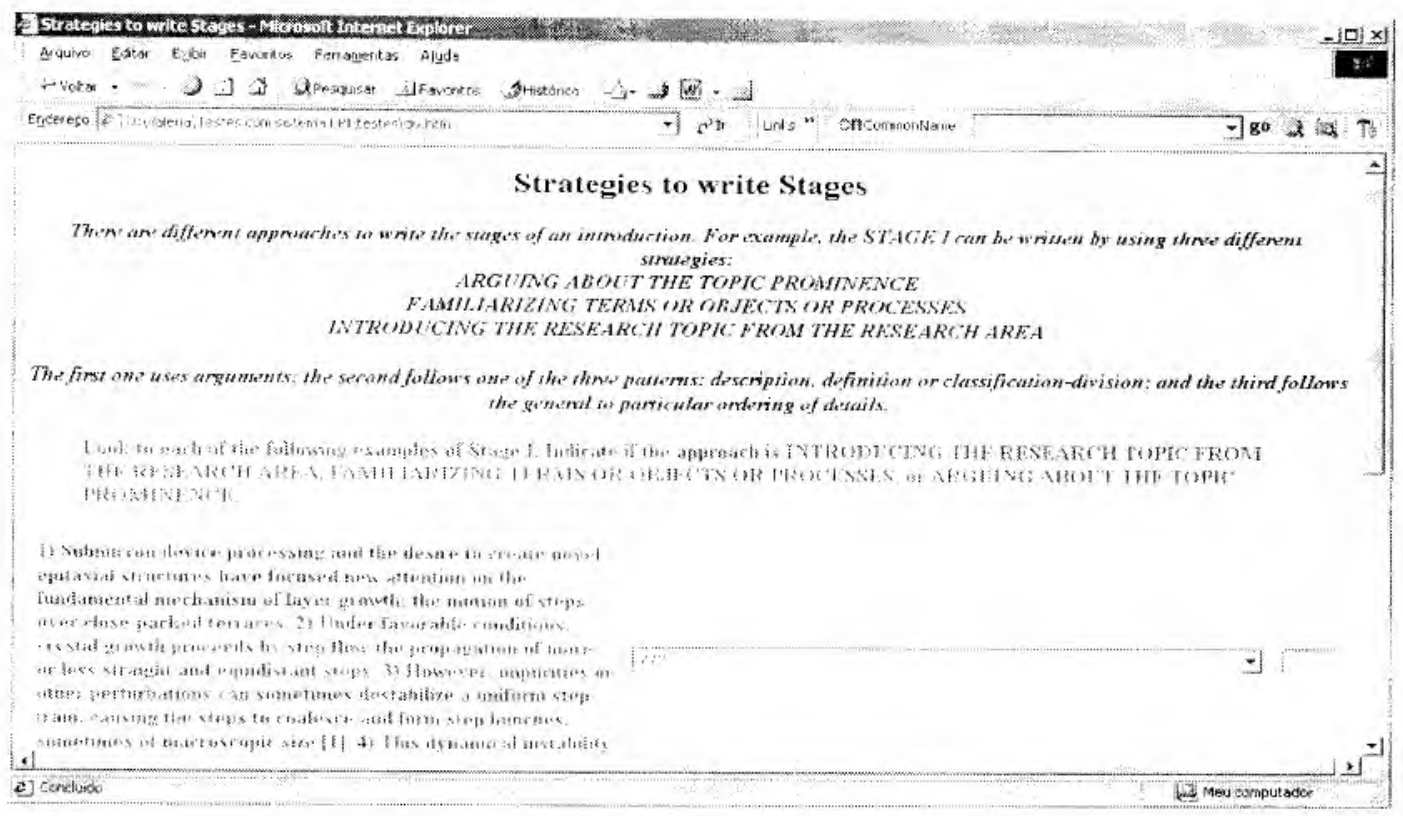

Figura Befi: Strategies to write Stagers

EReformulating text-Microsof Internet Explorer

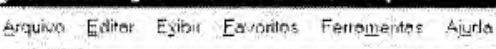

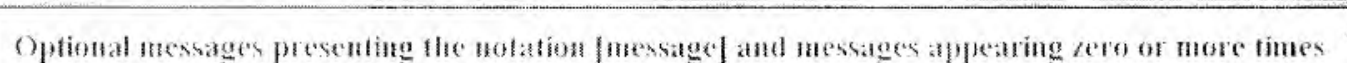

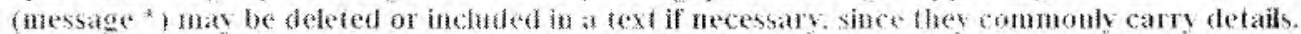

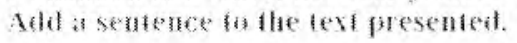

Iype your answer and click on "Cleck".

\section{Reformulating text}

Cofsider the text:

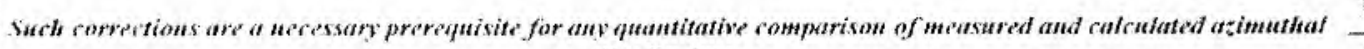
distributions:

Multifragment aimuthal cametation functians provide a potentialty poverfut probe for intermediate energe heasy ion dhamics.

Enlike a;imuthal distributions, these rorrelation funrtions do not require any knowledge of the reaction plane f12].

1 direct ronsequence of this fact is the rircumbution of the need for any went-by-prent estimation of the reaction plane and the assoriated corretions for dispersion of this plate about the true reaction plane.

Figura B.7: Reformulating Texi 


\section{Apêndice C}

\section{Exame formal realizado em setembro}

\section{de 2000}

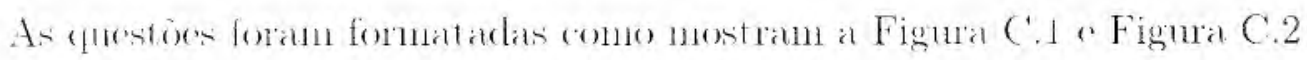

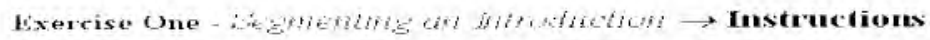

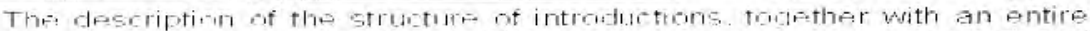

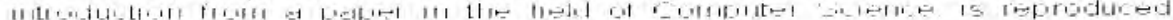
here first read thom. then arestar the questions

The introduction of a research paper can be divided into 8 parts, or stages.

In STAGE I, the writer establishes a context, or frame of reference to help readers understand how the research fits into a wider field of research. In STASE II, the writer reviews the findings of other researchers Who have already published in hisher area of interest.

In STAGE III.

* the writer indicates that previous literature described in stage II is inadequate because an important aspect of the research area has been ignored by other authars; or

* the writer indicates that there is an unresolved conflict among authors of previous studies concerning the research toplc; ar

- the writer indicates that an examination of the previous literature suggests an extension of the topic or raises a new research question not previously consldered by other workers in the field.

STAGE IV formally announces the purpose $(\$)$ of the reseanch. STACEV V, the methodology, describes the steps followed in conducting the research. In STAGEVI, results, the writer preserits the maln findings of the research. STAGE VII indicates posslble benefits or application of the research. Sometimes, stage VII is combined with stage $\mathrm{W}$ in the same sentence. FInally, in STAGE VII, the writer preserits the structure or layout of the paper, indicating the sections and briefly commenting on them.

Surne of the stages are optional arid may depend on the journalconference style and length of the article.

However, the general plan given in the reacting frame is very common.

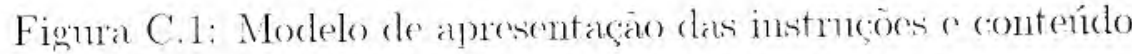

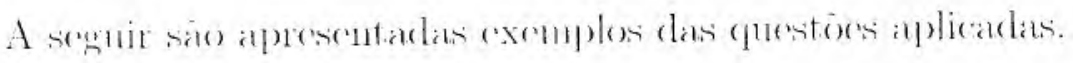

Exercise One - Segnicuting an Introduction - Instructions 


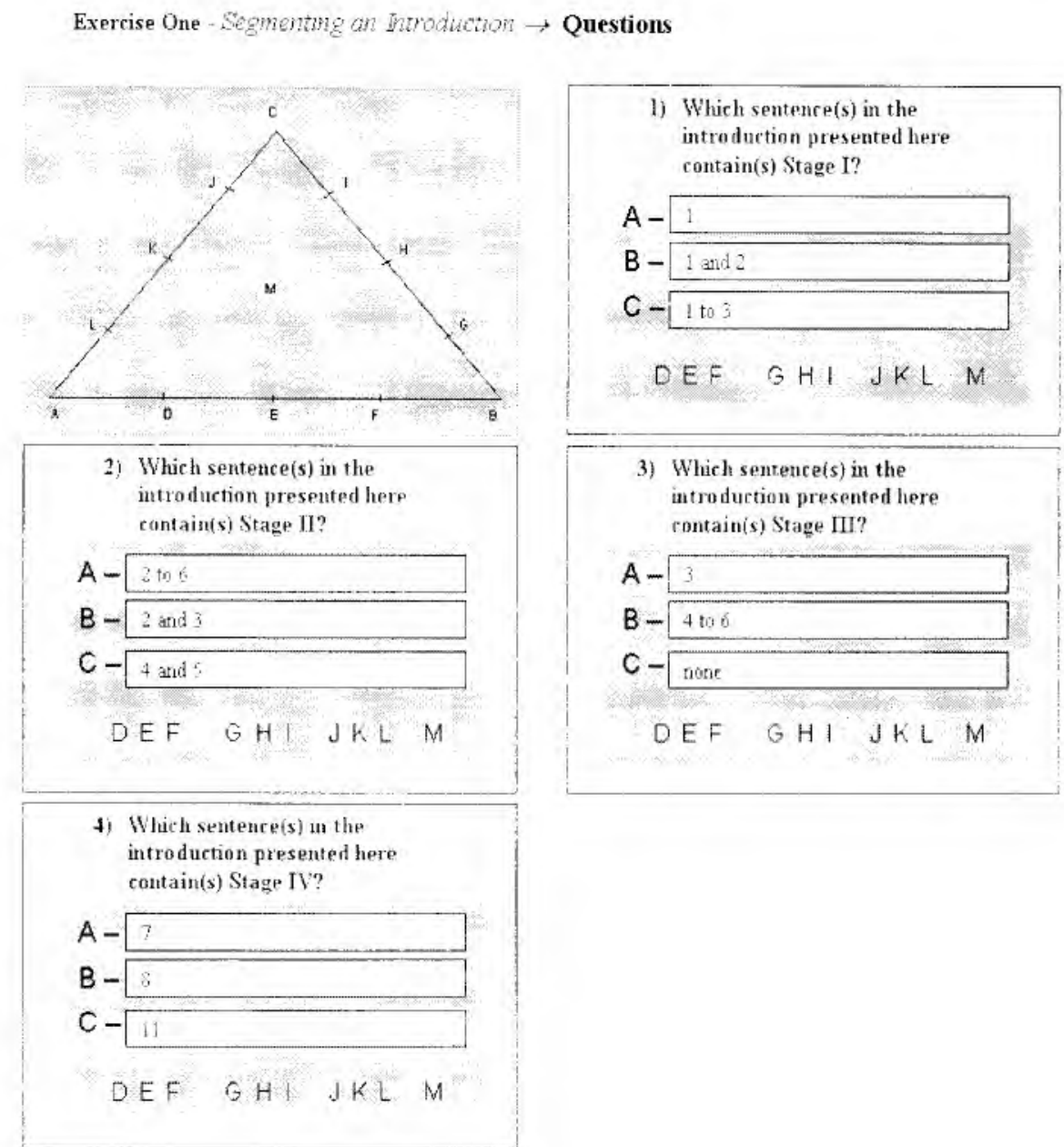

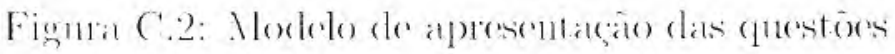

The description of the structure of introductions, together with an entire introduction from a paper in the field of Computer Science, is reproduced here. First road them. then answer the questions.

The introduction of a researed payper can be divided into \& parts. or stages. In STACE I. the writer establishes a context. or frane of referemer to help readers mudersfand how the research fits into a wider fiedel of rescated. In STAGE II. the writer reviews

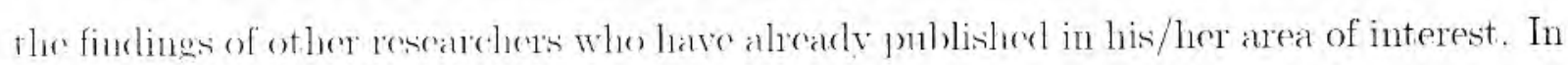
STACEF IJI: * the writer inclieates that previons literature deseribed in stage II is inadeguate becanse an inportant aspecti of the research area las been ignored by other authors; 
or * the writer inclieates that there is an muresolved conflict anomg anthors of previons

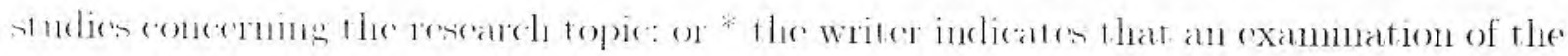

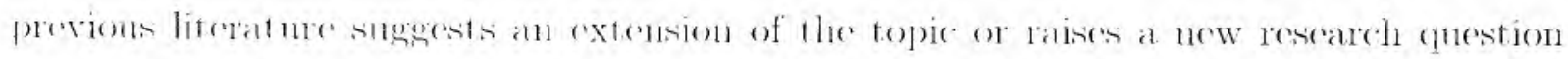
not previonsty considered be of here workers in the fiold. STAGF IV formally annomes

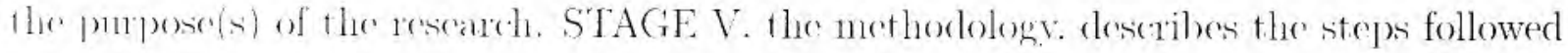
in ronducting the researeh. In STAGE VI. results. the writer presents the main findings of the rescrach. STAGE VII indicales possible benefits or application of the research. Somenimen. stager VII in combined with stage IV in the sane sentenee. Finally, in STAGE

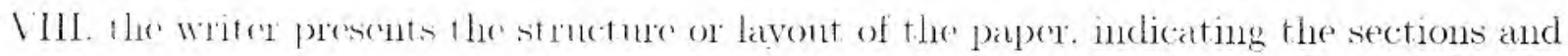
briefly commenting on thene. Some of the stages are optional and may depend on the

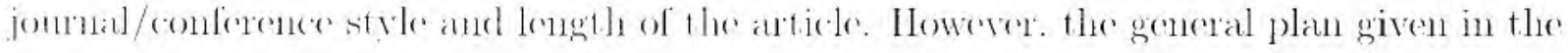

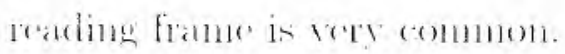

\section{Exercise One - Segmenting an Introduction - Questions}

1) Which sentenerest in the introduction presented here contamis) Stage I?

$a-1$

b -1 and 2

(1. $-1+03$

2) Which sentenes(s) in the introduction presented here contain(s) Stage II?

(1. 2106

1) -2 and :3

(c- 4 alid 5)

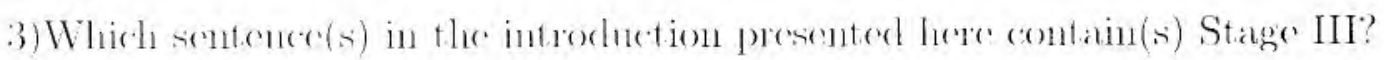

a1 -3

1) $-1+06$

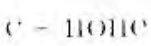

4) Which sentenes(s) in the introduction presented here contain(s) Stage IV?

$a-7$

b. -8

c. -11 
Exercise Two - Rrassembling an Introduction - Instructions

The following sentences in block were taken from an Introduction from a paper in the field of Statistics. The sentences are given here in a scrambled order. The title of the paper is also given. First read them, then answer the questions.

Some becond ()rier Asymptotic's in Exponential Family Non-Linear Regression Norkels

1. In this paper. We apply the geometric franework of Amari (1985) and tensor uotation to study some second oreler assmptoties for estimator in exponential fanily nomlinear regression models.

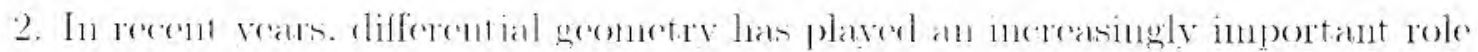

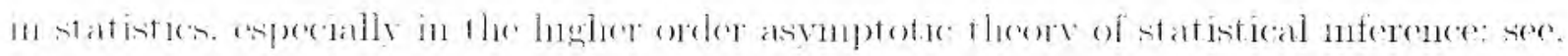

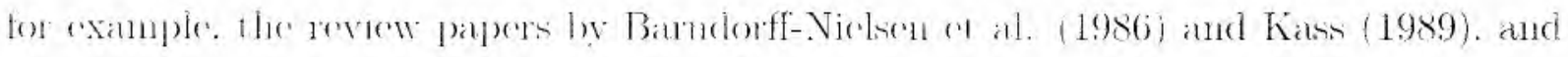
looks be Anary (1985) and Murray of Rice (19993).

3. Wr present a set of robomalse and relatively simple regularity conditions. with which to study astruptotics from a geometric point of view in regression models. ()u principal conditions are that geonertice quantities such as the metrice curvature.

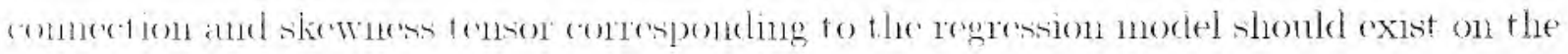
arrage of observations.

4. Howerex. the asymptotics redated to the comvatures for regression models have

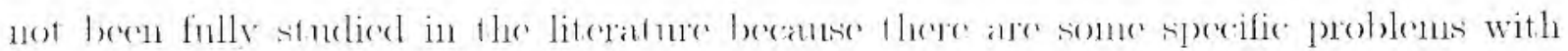

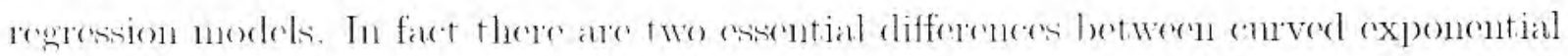

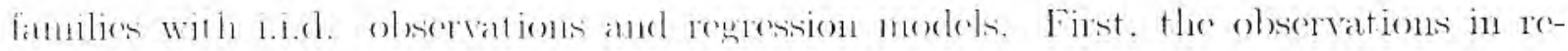

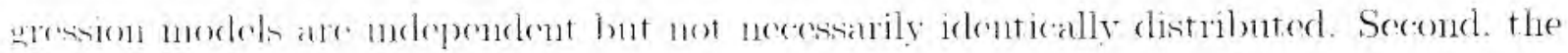
mainfold eorresponding to a regression ehamges as the sample size in 11 increases. while for the i.i.r. (ase studied by Efron (1975) and Amari (1982a) the geometric structure is an tually based on one of the i.i.l. observations (Anari. 1985). Therefore the asymptotics

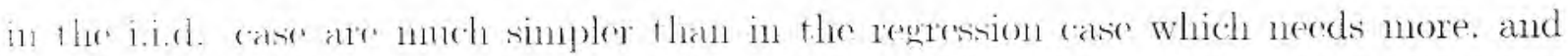
usmally severe. regularity conditions. 
5. Efron (1975) and Amari (1982a.b) introduce a differential geometric frame-

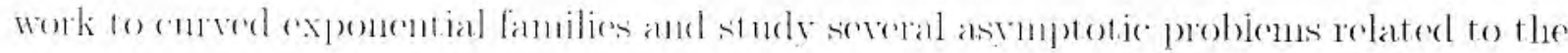

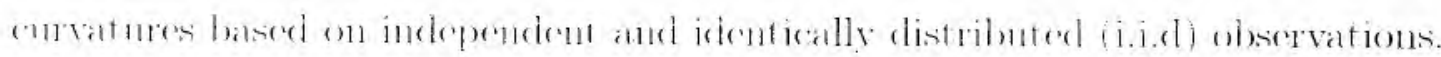

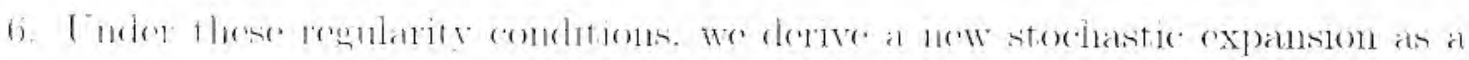
toxel to study the astumptoties for exponential family monlinear regression models. Om results may be regarded as fiuther derelopments of previoms work by Efron (1975). Efron (t Hinkley (1978). Amari (1982a.b) and Wei (1991). 7. Section 2 describes the geometrie aspects of exponemtial family nomlinear regression models based on the geometric franework of Anari (1985). Section 3 derives a new stochastie expansion for first order

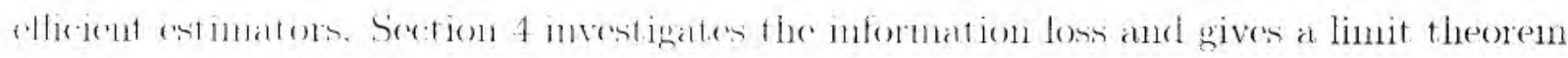
anmered with the observed information and the expected information. In Section 5 .

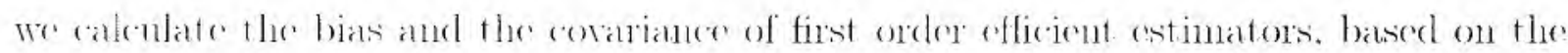

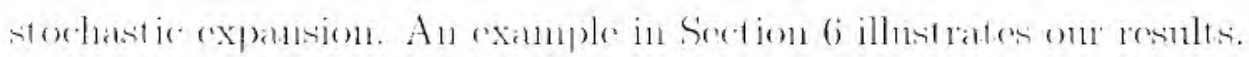

\section{Exercise Two - Reatsicmbling an lutroduction - Questions}

1) Which block in the introduction presented liere is the most appropriate to appear as the first one.

$$
\begin{aligned}
& a-4 \\
& b-2 \\
& (-5)
\end{aligned}
$$

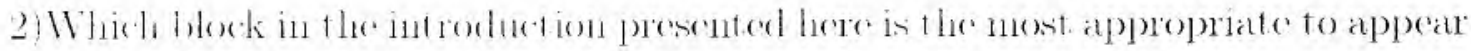
as the sereonet one.

$$
\begin{aligned}
& \text { a. }-4 \\
& 11-7 \\
& \text { (.) - 3 }
\end{aligned}
$$

3) Which block in the introduct ion presenter here is the most appropriate to appear as the thinel one.

$$
\begin{aligned}
& a-4 \\
& b-6 \\
& \text { (a) }-3
\end{aligned}
$$


4) Which bleck in the intredention presented here is the most appropriate to appear as the founth one

$$
\begin{aligned}
& a 1-1 \\
& b-1 \\
& \text { c. }-6
\end{aligned}
$$

5) Which block in the introduction presented here is the most appropriate to appear at the fifth one.

$$
\begin{aligned}
& a-3 \\
& 1)-5 \\
& (1-6
\end{aligned}
$$

Exercise Three - Nom Pluases - Language (omentions - Instructions

Several ways to write nom phrases, together with an example of a Stage I from an introduction from a paper in the field of Physics, are reproduced here. First read them. then answer the questions.

Statements in the Stage I of an introduction tend to be general in nature. Instead of referring to sperifie things. they olten refer to entire classess of things. When von write sentenees that contain nomus referring to an entire class of things. von should use generie

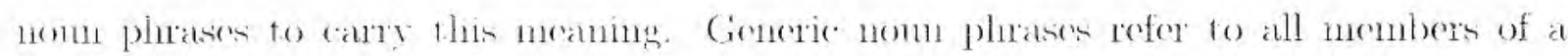
particular clatsis of hiving thingst.

In English there ane different ways to write genorice nom phases. If the nom

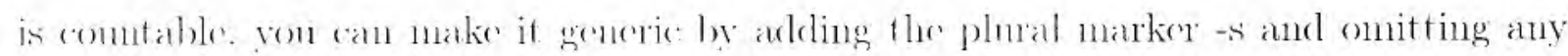
anticle. on be using it in singular form with the inclefinite anticle a or ane. When the nom

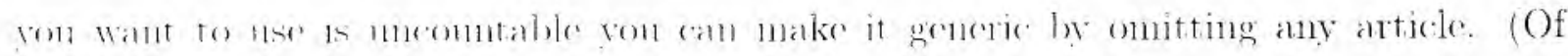

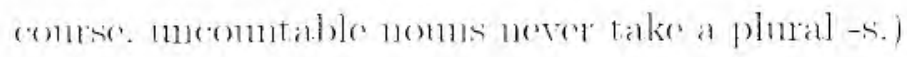

Later in the sotting. you will probably find it necestary to refer to specific items and concepts in oreler to nove the reader from the general area toward your specific topic. This requires the nse of specific nom phrases - that is. nom that refer to particular, individual members of a class rather than to the class as a whole. In English, nom with 
this moming (an be written in seremal wats:

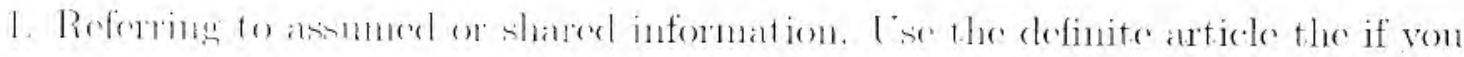

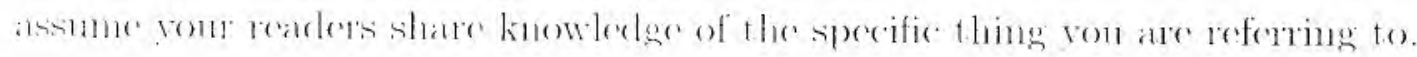

2. Pointing lack to olel infomation. Use the definite article the when referring to a specific thing which vou have aldearly mentioned (the first mention usually uses the indefinite article a/an)

3. Pointing forwarel to sperifving information. Exe the definite article the when

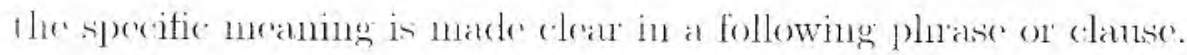

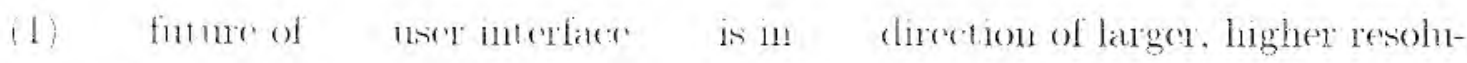

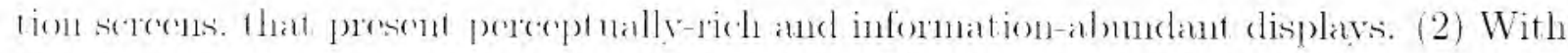
such elesigns. Worrisone flood of information can be tumed into productive river

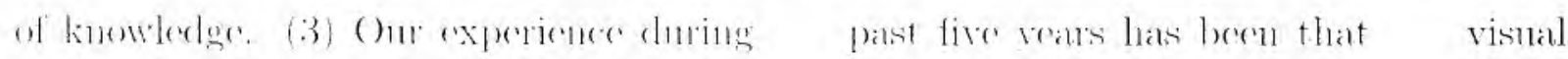
gunery formulation and visual display of results can be combined with sucessful strategios of direct manipmlations. (4) Human perecptnat skills are cunite remarkable

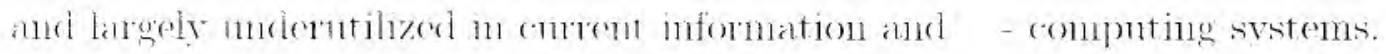

\section{Exercise Three - Nom Phrases - Language Conventions - Questions}

1) Which is the serpenes that fills in the blanks of soutenes 1 .

$a-a / a 11,+13 e . \quad . a / a n$

1)- the the

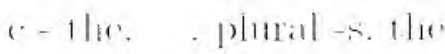

2) Which is the secpuenere that lith in the blanks of sentemere 2.

a-the. a

b) - a/ant. the

(c)

3) Which is the sepenenere that tills in the blanks of sentenee 3 .

a- . the, . the

b - the.... the

(c- theca a/an. a/ant the 
1) Which is the serpuenee that lills in the blanks of sentenes 4.

it - Huc

n. - Thw

(.) .

Exercise Four - Stagr III (Language Comroutions) - Instructions

Different ways to write the Stage III of an introduction, together with seven examples of Stages III from papers in the field of Physics, are reproduced here. First read them, then answer the questions.

Signal Worchis

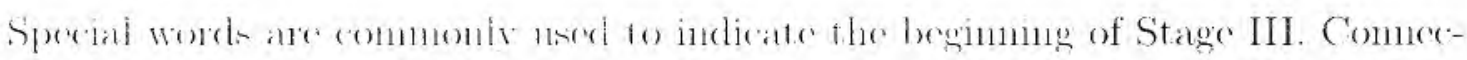

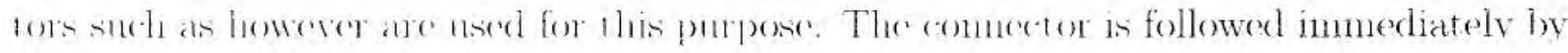

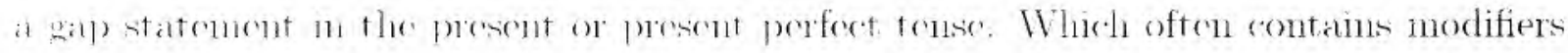
sherls as fert. liftle. or no.

Signal word + Gap (present or present perfect) + Research topic:

Suberdinating conjunetions like while cant also be used to signal Stage III. If you

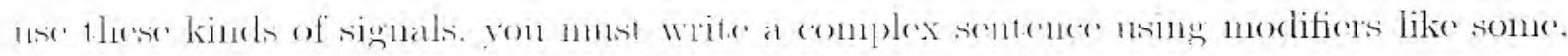

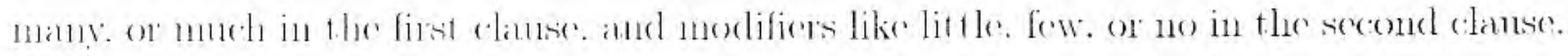

Signal worel + Previons work (prescent or present prefect) + Gayp + topic

1. (omprufers lawe proven adept at all manuer of data processing. paper is still gencrally prefered oxer PDAs and portable compunters for note-taking.

2. the increasing number of new devices entering the matket allowing the capture ore recorling of information (whether it be matrs on paper, secue, somend or moving inages). Hhere has been little stredy of when and why people want to do these 
kutelo of activities. Honovation.

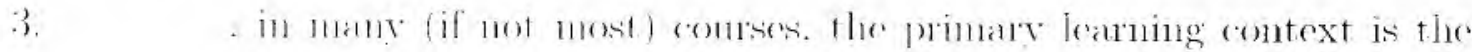
(tastionom.

4. there exist a momber of ereseated systemes that enuploy varions combimations of pen-hased devices in enjunetion with andio and/or video ([1]. [2]). the nse of digital video as a nedinun that noels in nectings can interact with and incorporate into then notes has not beren explored.

5. . one is left with a long linear strean of andio and cannot randomly aress indivichat thoughts.

6. 1here has been a lot of researeh artivity in the area of antomated

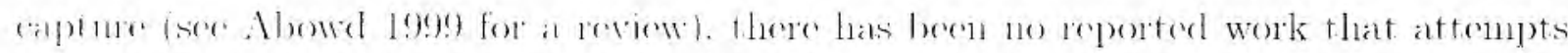

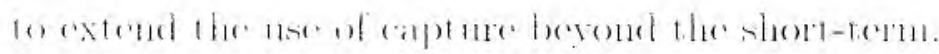

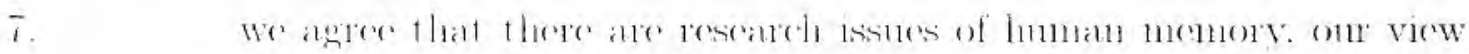
is that the capturing and salvaging of meetings needs to be mulerstood within the social and organizational context of the langer work procestist.

Exercise Four - Stagr III (Lamgnge Conventions) - Questions

1) What is the signal word appropriate to complese the blank space of example 1 ?

a- Howerer

1) - Althorght

(c- B B11

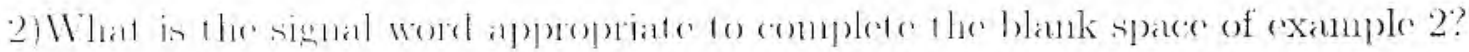

i) - Bı11

h- Despite

(.- Howerer

3) What is the signal worel applopriate to (omplete the blank space of example 3 ?

at - Bnt

1) - Althorigh

(- - Howere'

4) What is the signal word appropriate to complete the blank space of example 4 ? 
a) - While

1) - Bu1

c. - Hexrorer

5) What is the signal worel appropriate to complete the blank spare of example 5?

a- Althorgh

1, - B B11

(1.- Henerer

6) What is the signal worel appropriate to emplete the blank space of example 6?

a- But

1) - Thomgh

1. Howros

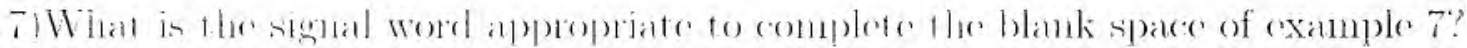

a. - While

1) - B311

1.- Howris

Exercise Five - Stage IV (Languagr Conventions) - Instructions

Different ways to write the Stage III of an introduction, together with seven examples of Stages III from papers in the field of Physics, are reproduced here. First read them, then answer the questions.

() riventation and Truse

Yon mas write the statement of pmesese (Stage IV) from one of two altexuative orientations: 1 - The orientation of the statement of pupose may be towards the report

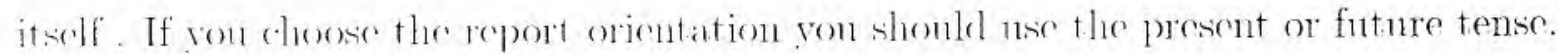

Report oriontation + Main Verh (present or future) + Rosearch question

2 - () the orientation of the statement of purpose may be towards the researeh 


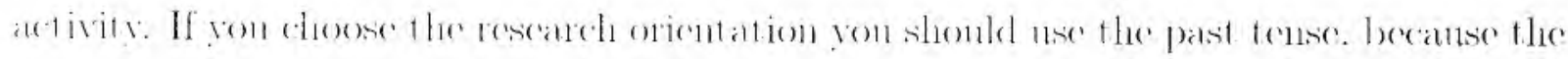
mestach activity has aldeady berom completerd.

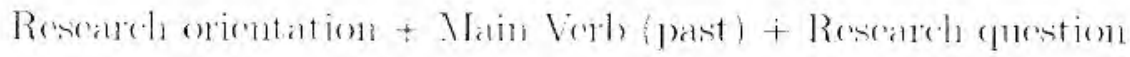

1. The thesis of this paper is that software enginerring advances are repuired to

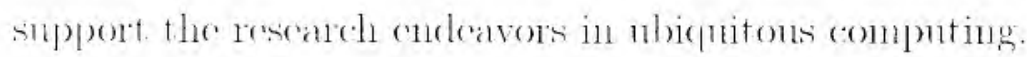

2. In this dissertation. we describe a technigne for dvnamically gromping digital

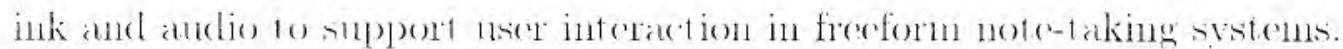

3. This report examines the pratice of anuotation an a particular sitnations the

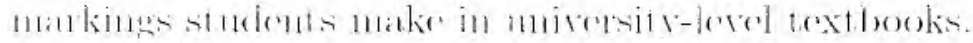

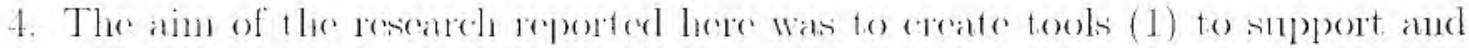
(anpture the fres-flowing artivition of meetings and (2) to utilize the captumed multimediat uesting records offectively in the largere work processes in which the neetings ane einherkterl.

5. In response to this. We designed a study to andrese people sinformation capture artivities.

6. This paper presents a spoech interface for a hand-held computer that allows

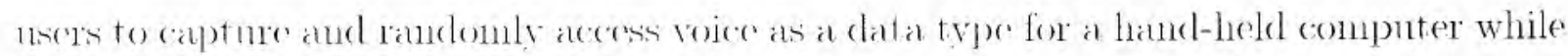

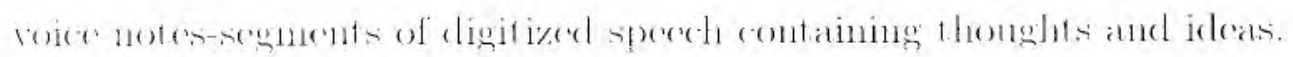

7. The purpose of the stuely reported in this paper is to begin to explore work

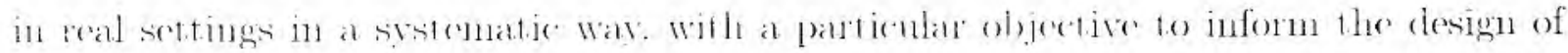
session manlagement models.

Exercise Five - Stage IV (Lamgnage Comventions)-Questions

1) Which example(s) of Stage IV is (are) written from report orientation?

a -1 to 4

1) -5

1. $-110 ; 3$ 


\section{Apêndice D}

\section{Exame piloto realizado em Fevereiro}

\section{de 2001}

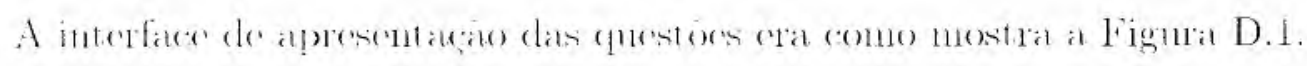

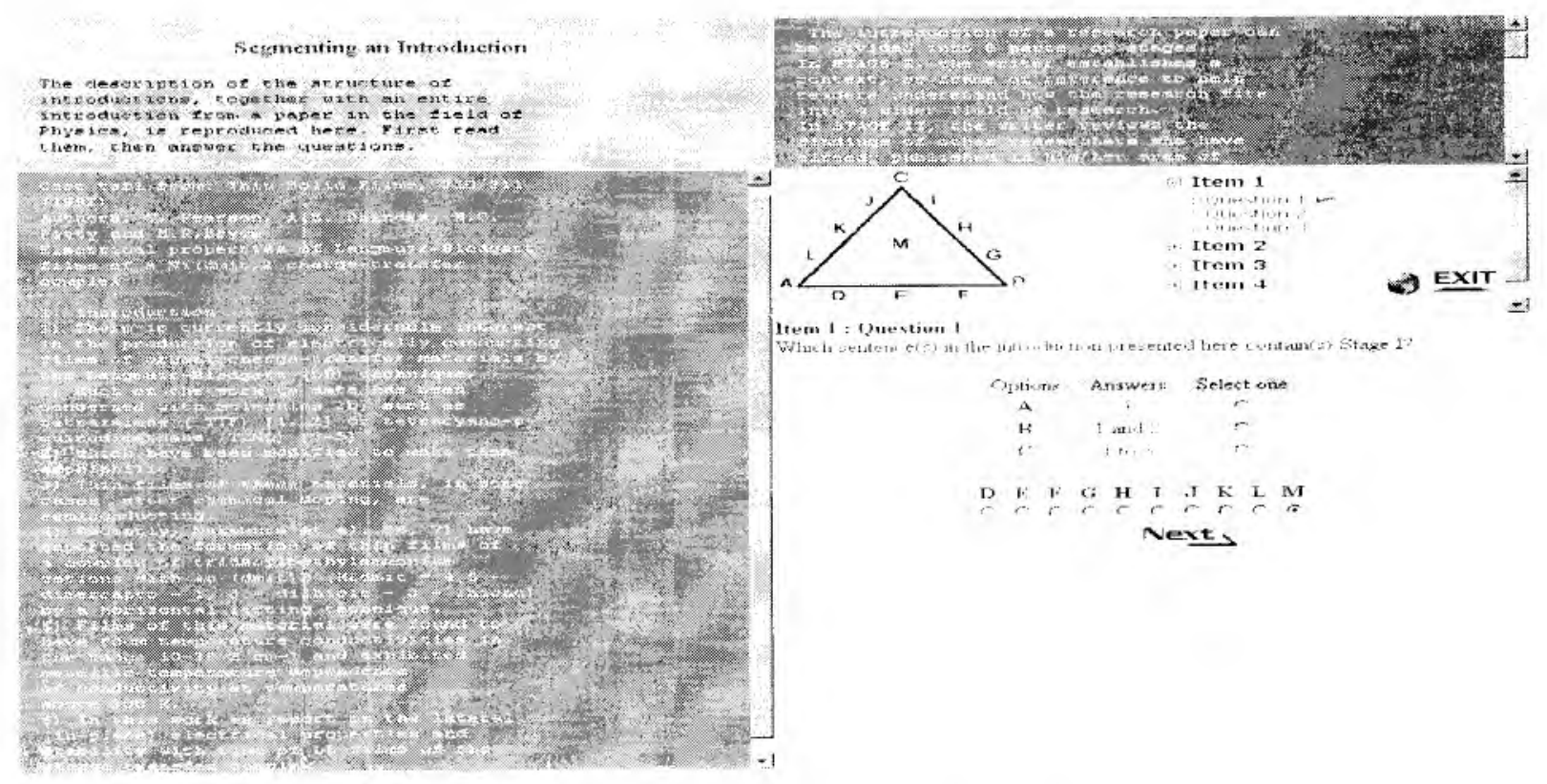

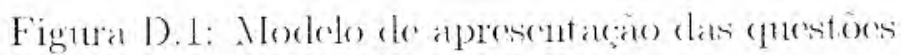

A seguix sao apresentadas exemplos clas questoes aplicadas.

1 - Divide and Classify the parts of an Introduction Instruça 
The description of the structure of introductions, together with an entire introduction to a paper in the field of Computer Science, is reproduced here. First read them, then answer the questions.

Eaplinacuro

The introduction of a research pajer sau be divided into 8 parts. or stages.

In STAGE I. the writer establishes a context. or frane of reference to help readers nuderstand how the rescareh fits into a wider fiekd of research. In STAGE II. the writer wevers the findings of oflere researeders who have alreaty published in his/her area of interest.

II STICIE III:

* the writer indicates that previons literatume deseribed 111 stage Il is madecflate

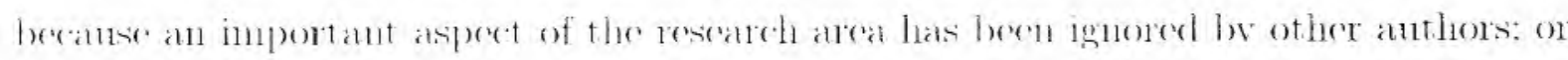

* the writer indicates that there is an muresolved eonfliet anomg anthors of previous stmelies concerning the rescanch topie: or

* the writer indicates that an examination of the previons literature suggests an extension of the topic or raises a new research question not previonsly considered by other workers in the fichd. STAGE IV formally amomess the pmpose(s) of the research. STAGE V: the nethodology: describes the steps followed in conducting the research. In STAGE VI. restuts. He writer presents the main findings of the research. STAGE Vll indicates possible benefits or applieation of the researelt. Sometimes. stage VII is combines with stage IV in the sane sentence. Finally. in STAGE VIIt the writer presents the structure or layout of the paper. indieating the sections and briefly commenting on them.

Some of the stages ane optional and may depend on the jommal/conference style and length of the article. Howeres. the general plan given in the reading frame is very (อmกnoti.

Teito 
Lectures Notes in Artificial Intelligence 1010, pp. 229-240

Learning to Improve Case Arlaptation by Introspective Reasoning and CBR

\section{David B. Leake, Andrew Kinley, and David Wilson}

Introduction

1) Cascobased reasoning (CIBR) systens solve new problems by retrieving prior solutions of smilar previons problems and perfoming (aks adaptation (also called case morlifications to fit the retrieved adses to the new situation. 2) Althongh much progress has been made in methods for case retrieval. both the Anerican and European CBR, communition lave identified casse adaptation as a part ienland challenging open problem for

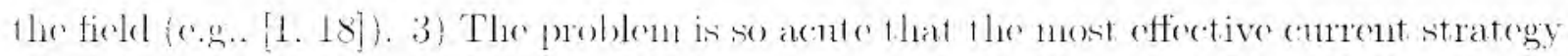

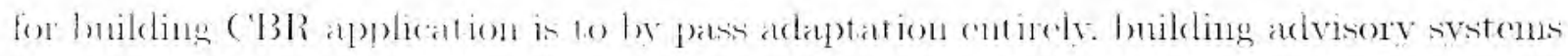

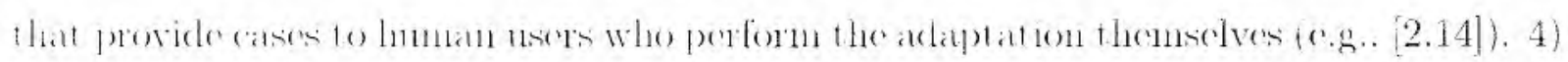

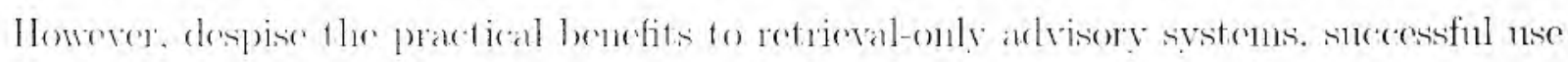

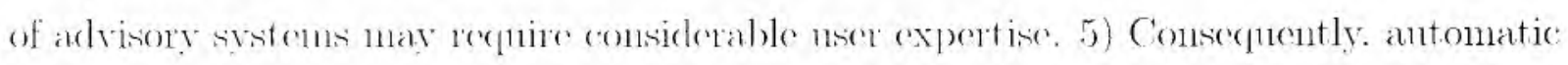
asc arlaptation is inportant from a practical perspective. nof only to enable CBR svstems to antonomonsly but to enable thene to aid naijve nsers. 6) Likewise. as we disconss in [19]. increased nuderstanding of the case adaptation process and the knowledge required is also important from a cognitive modeling perspertive. as a step towards nuderstanding how humans adapt cases when they reasons from prior (pisodes. 7) This paper describe rescarch based on chamaterizing case adaptation kuowleche by decomposing it into two parts: (1) a sumall sot of alstract strucetural transformation (c.g. [5.9]). and (2) memory seared strategies for finding the information needed to apply these transformation. This

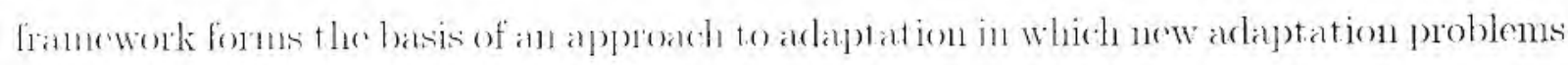
are solved by first selecting a transformation indexed under the type of problem motivating adaptation, and then performing int respective reasoning aluont how to strategically search menory for the information uereded to apply the transformation [17]. Not only does this approact provide increased flexibility in finding needed information, but serves as a fom dation for learning to improve adaptation performane for experience: A case of this process can be stored as and adaptation ease and used in fut une casc-based reasoning about 


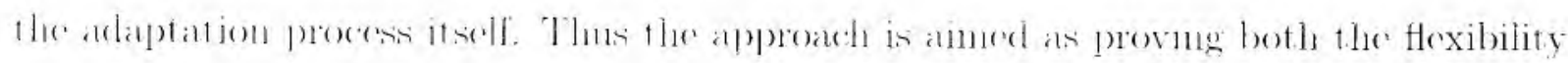
fo deal with nowed rase adaptation problems and aclaptation abilities that improwe with experiones. We begin be discossing the significance of the case ataptation problem for CBR and the tomets of one approath. We them smmmarize an initial implementation that

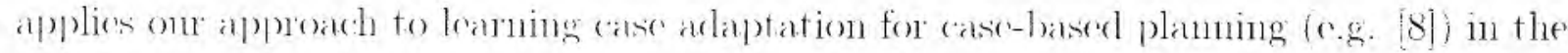
dinaster response plamning domain. Wo rose by highlighting lessoms learned and related rescarch on casce alaptation and memery seareds.

Questiofs

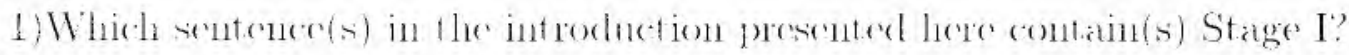

(1) 1

b) 1 and 2

(.) 1107

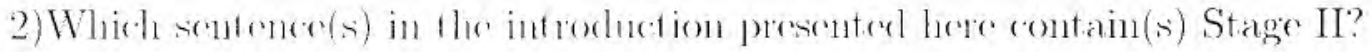

a) $2+06$

1.) 2 and! :3

(.) 4 and 5

3) Which sentence(s) in the introduction presented here contain(s) Stage III?

a) 3

1) 4 to 6

(c) 110110

4) Which senteners) in Here introched ion presented here contain(s) Stage IV?
1) 7
b) 8
(.) 11

2 - Classify and Rearrange the parts of Introduction Itistrucian

The following sentences in block were taken from an Introduction to a paper in the field of Statistics. The sentences are given here in a scrambled 
order. The title of the paper is also given. First read them, then answer the questions.

Trite

Some Second (Oeder Asymptoticts in Exponeutial Family Non-Linear Regression Models

1. In this patpere we apply the geometric franework of Anari (1985) and tensor metation to study sonne second oreter ansmptotice for estintator in exponential family molinear regressou mortels.

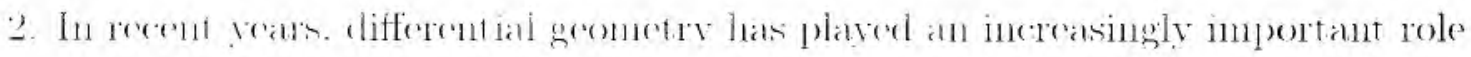
in statistics. especially in the higher oreler asymptotice theory of stat ist ieal inferenee: see,

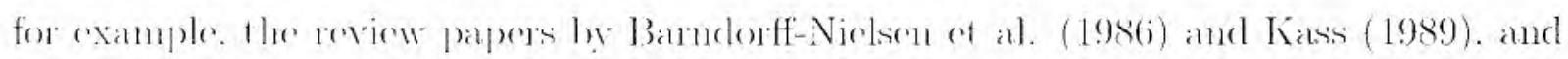
lowks by Amary (1985) and Murray (d Rice (1999:3).

3. We present a sol of reasomable and relatively sinnple regularity conditions, witl wheld to stucly asimptotice from a geometric point of view in regression models.

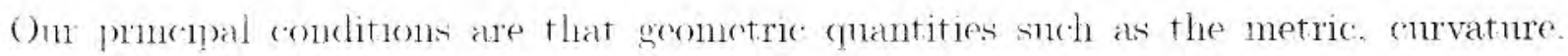
comection and skewness tensor corresponding to the regression model shond exist on the arerage of observations.

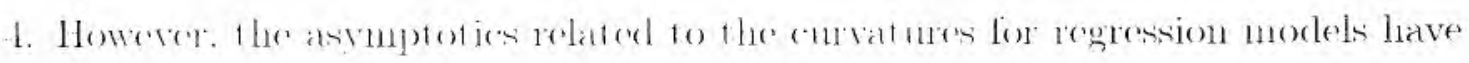

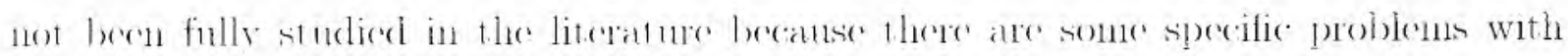

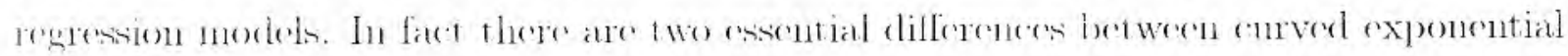
fantiles with i.ide observations and regression moleds. Fitst. the olservations in re-

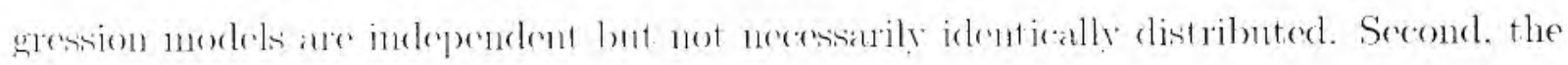
mainfold corresponeling to a rogression changes as the samplo size in $n$ inereases, while for the i.i.d. case studied hy Ffrom (1975) and Amari (1982a) the geometrie structure is an thally hated on one of the i.i.cl. observations (Anmi. 1985). Therefore the asymptotics in the i.i.d. case are much simpler than in the regression case which noeds more. and

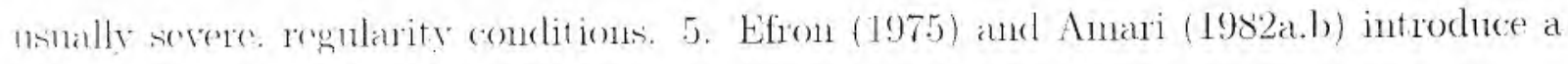
(litferential geonetric framework to (nuved exponential fanilies and study several asymp- 


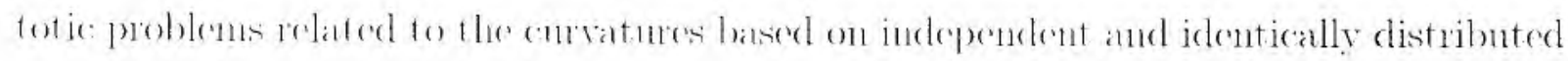
(i.i.d) olservations.

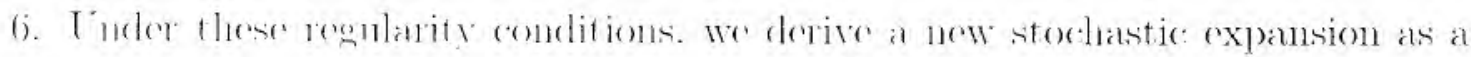

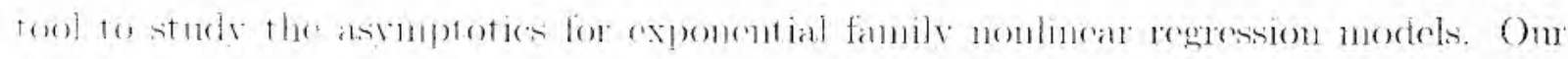
lesult may be regarded as funther developments of perions work by Efron (1975). Efron d. Hinkley (1978). Anlari (1982a.b) and Woi (1991).

7. Section 2 describes the geometric aspects of exponential family nonlinear regression models based on the geonetrie franework of Antari (1985). Section 3 derives a new stochatistic expatusion for first arder efficient estinnators. Section 4 investigates the

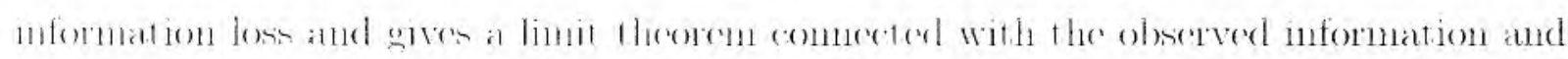

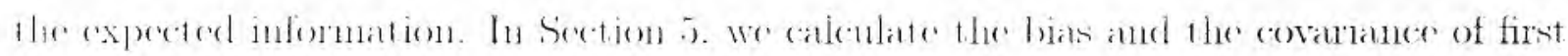

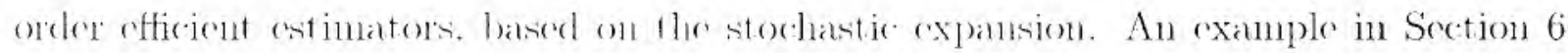
illustrates our results.

Questoes

1) Which block in the introduction presented here is the nost appropriated to appear as the first one?
a) 4
(1) 2
(c) 5

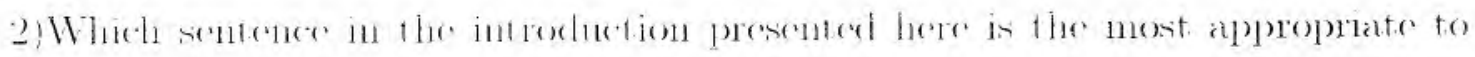
apreate as the second one.
a) 1
1) 5
(.) 3

3) Which sentenee in the intreduction presented here is the mest appropriate to apperay as the thincl one.
a) 4
b) 6 
(.) 3

1) Which sentenes in the interhetion presented lewe is the most appropriate to apperar as the fomptlo ome.
a) 1
b) 4
(.) 6

5) Which sentence in the introduction presented here is the most appropriate to

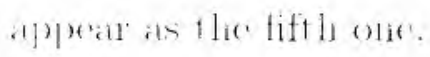
a) 3
b) 5
(.) 6
3 - Language Conventions - Signal Words
Instruscios

Different ways to write the Stage III of an introduction, together with seven examples of Stages III to papers in the field of Computer Science, is reproduced here. First read them, then answer the questions.

\author{
Explinacias \\ Signal Workts
}

Spereal werds are commonly used to indicate the beginning of Stage III. Commes-

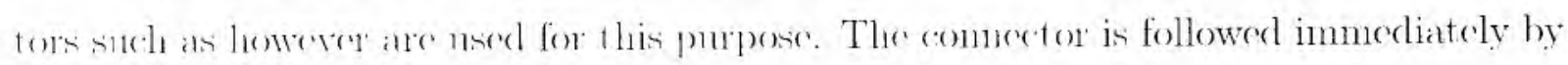

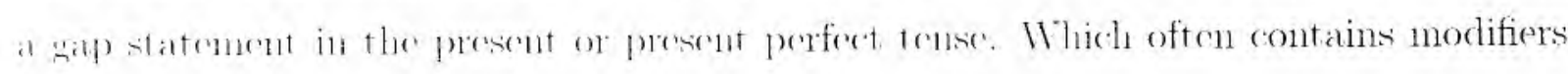

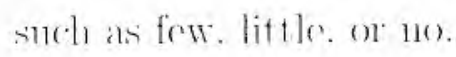

Sighal word + Galp (p)resent or present perfect) + Research topic:

Suborelinating conjunctions like while can also he nised to signal Stage III. If you 


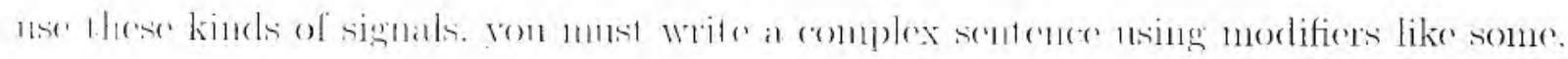

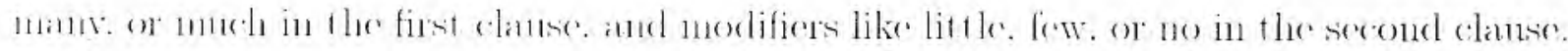

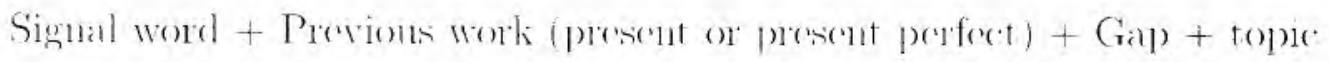

Texto

1. Gomputers have proven adept at all mianner of data processing. paper Is still getuerally preferred oxer P'DAs and portable connputers for note-taking.

2. The increasing number of new deviens entering the market allowing

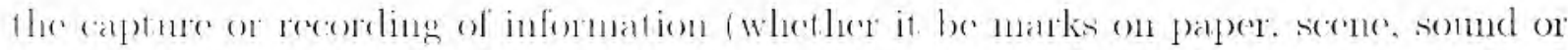

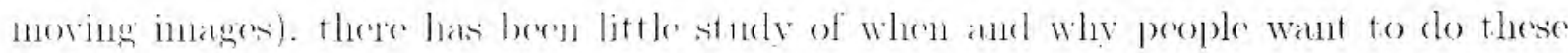
kinds of antivition. inmoxations.

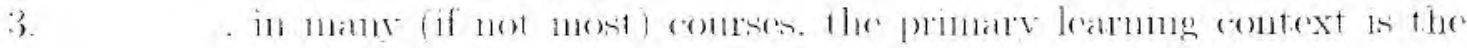
(Mistorin.

4. there exist a number of rescarch systems that employ varions combinations of pen-basod derices in coujunction with andio and/or video ([1]. [2]). the use of

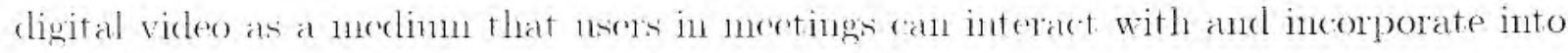
their notes lato not been explored.

5. . one is lefi with a long lonear strean of andio and annot randomly areses individual thonghts.

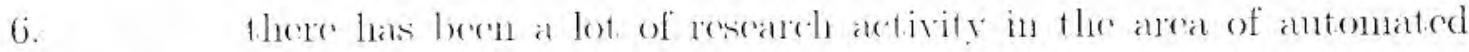

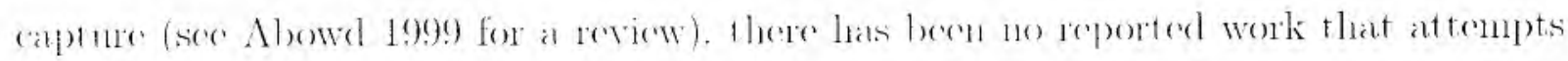

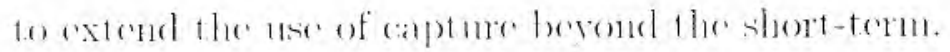

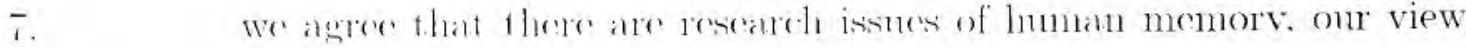
is that the cayturing and salvaging of mereings needs to be nuderstood within the social and organizational eontext of the langer work processes.

Questrifs 
1) Which is the signal worel that fill in the blank of example l?

a) Howerer

hi) Alluomgh

(c) P 3111

2) Which is the signal word that fill in the hlank of (xample 2?

a) Bu1t

b) Despite

(c) Howrow

3) Wheth is the signad word that till in the blatek of example 3 ?

i) But

b) Althomgh

(.) Hoнеген

4) Whede is the signal word that fill in the blank of example 4"

(1) While

b) But

(c) However

5) Which is the signal worel that fill in the blank of example 5?

a) Althomgh

b) Bui

(-) How

6) Which is the signal worel that fill in the blank of example 6 ?

a) Bu1

1) Thomgh

(.) Howere

7) Which is the sigual word that fill in the blank of example 7 ?

a) White

b) But

(c) Howerers

4 - Strategies to write the Stages of an Introduction Instrulusion 
The different strategies to write the stages of an introduction together with examples of Stage I of an introduction to a paper in the field of Computer Science, is reproduced here. First read them, then answer the questions.

Explinarios

There are different appreaches te write the stages of an introduction. For example,

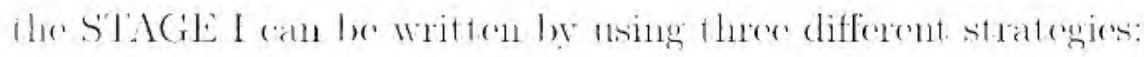

- argtting abot't the topic: proninence

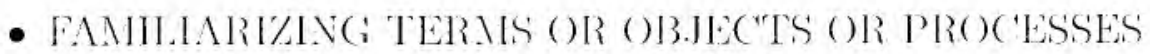

- INTRODUC CING THE RESEARC'H TOPIC FROM THE RESEARCH AREA

The first one uses alemententis: the serend follows one of the thee patterns: descrip1ion. clefinition or chasifiention-division: and the thimel follows the general to particular orclering of details.

Tirito

Lectures Nots in Artificial Inteligence 1010, pp. 279-240

Exitimple 1

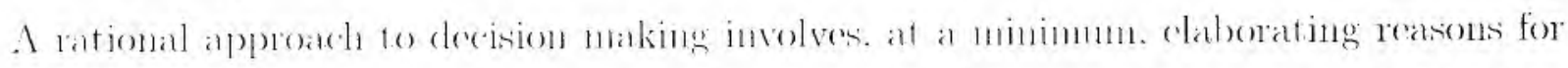
and against a proposed and ion. Since leatsons both for and against an action usmally exist.

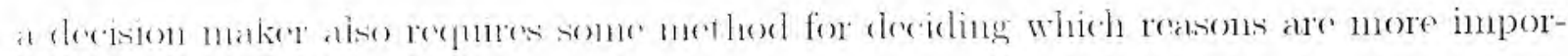
fant and shomld be able to explain the decision in terms of the reasons for it and a for the preforence.

\section{Examplo 2}

Rerently. there has been growing interest in the leanening franeworks using the knowledge

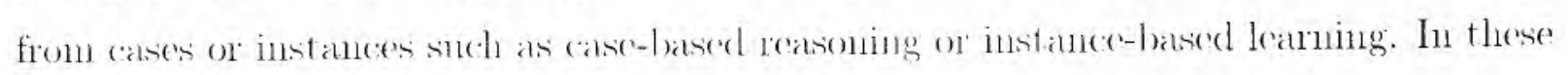




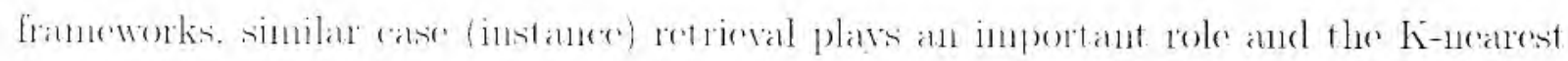

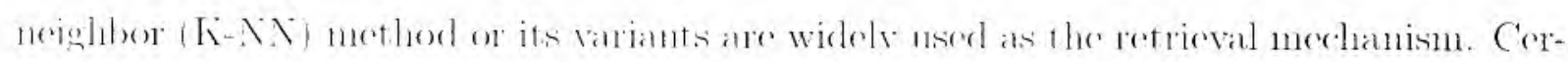

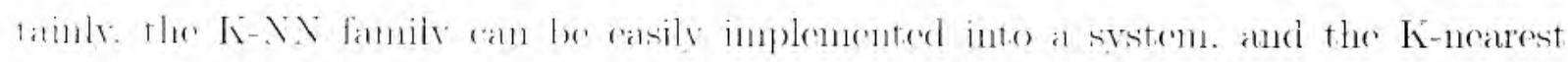

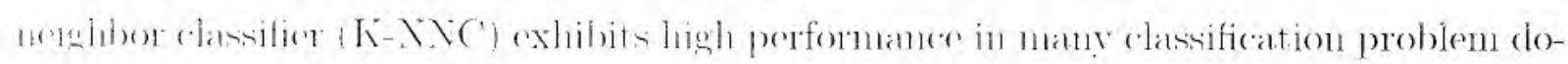
mails.

Examplo 3

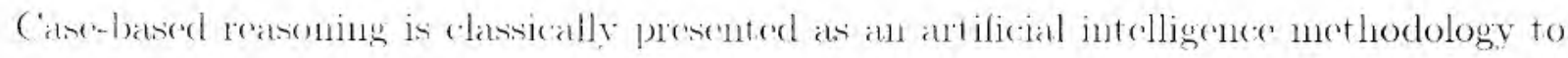
procens empirical knowlextge [1]. In oreler to achiere this goal, it stuclies how to design

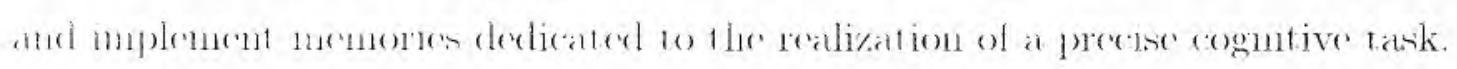

Qurestores

1) Which approach follows the example l?

a) ARCATNG ABOTT THE TOPIC PROMINE.TCF

b) FAMILIARIZING TIRAS ()R OB.JECTS OR PROCESSES

c) INTRODUCING THE RESEARCH TOPIC FROM THE RESEARCH AREA

2) Which approach follows the example 2 ?

a) ARGLING ABOUT THE TOPIC PROAINENC'E

b) EAMHLJARIZING TERMIS ()R ()B.JE(TS OR PROCESSES

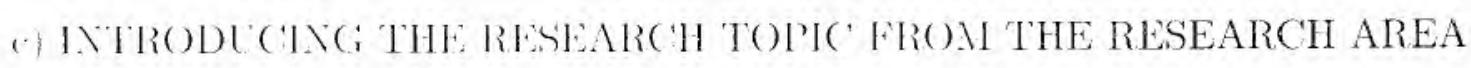

3) Whicte approate follows the rexample :3'?

a) AR(IINC: ABO)T THE TOPTC PROMINENCE

1) FAMIIIARIZING TERMS OR (OBJECTS ()R PROCESSES

(.) INTRODICING THE RESEARCH TOPIC FRON THE RESEARCH AREA

\section{5 - Summarization Deletion Operation}

Instruciou 
The description of a summarization operation together with an entire introduction to a paper in the field of Physics is reproduced here. First read them. then answer the questions.

\section{Extplicascin}

Jommal articles exlitors often establish a word limit for the text heing submitted. In order to satisfy such linitations we an perform several operations on texts. One of them is

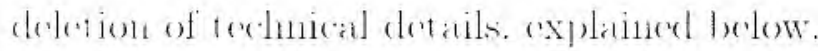

Somedines we can detete sontenees experessing definitions. exemplifications. partirolarizations. and ofleres. in oreder to obtain a shorter text. For example:

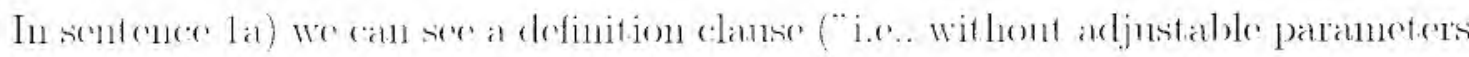
on empirical interaction potentials") that can be deleted to shorten the text. resulting the sintence 11$)$.

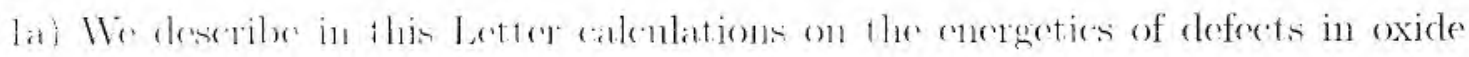
manrials performed entirely from first principles, i.e. withont adjustable parameters or ompirical interation potentials.

1h) Wo describe in this Letter calculations on the ruergetics of defects in oxide materiak performed entirely fiom first principles.

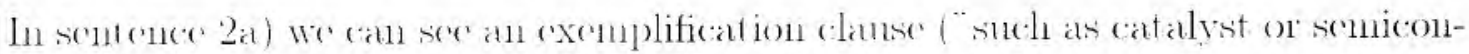

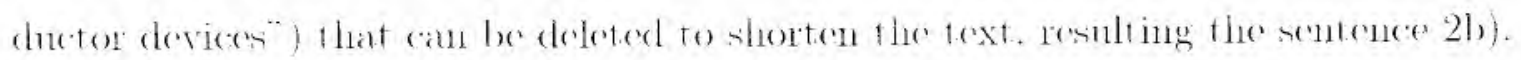

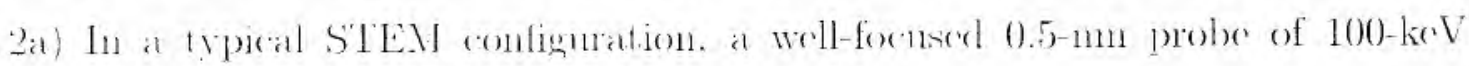

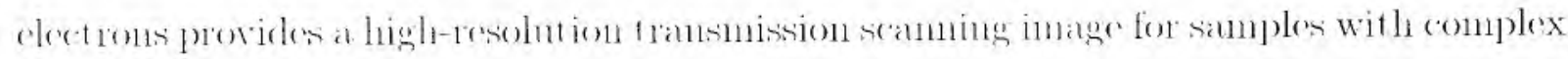

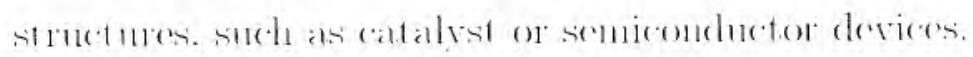

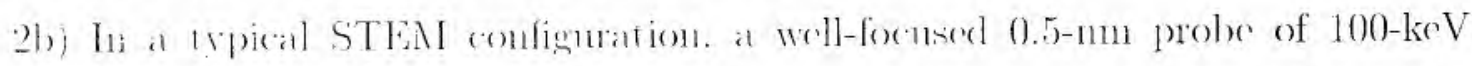
wert rons provides a high-1esolution transmission scanning inage for samples with complex strinctures.

In sentenes 3a) wo (an ser a particnlarization clanse ("specially for the fabrication and application of LD filus") that (an be deleted to shorten the text. resulting the sentence $31)$.

3a) However. homogeneons monolayers of polviners ane required for many purposes. 


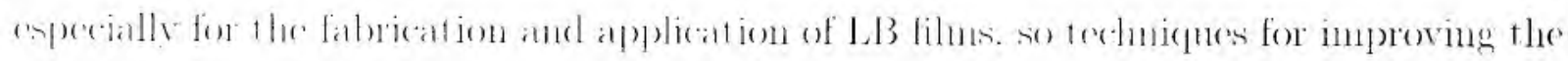
hemegeneits of polvmer momolavers are of interest.

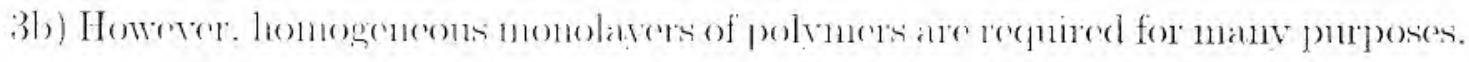

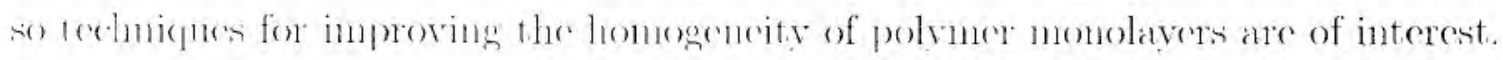

Alsos. we (an a dpply the deletion operation whene writing for knowledgrable andien-

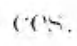

T+2:10

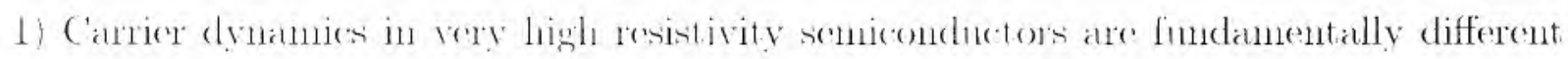

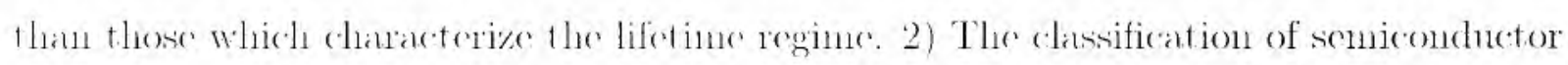

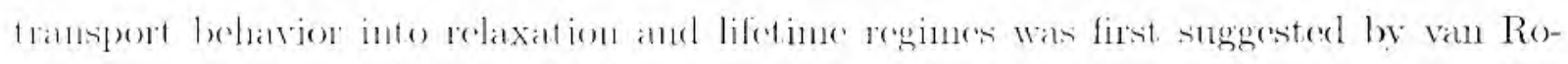
ostrock oxer thirty vears ago [1]. 3) The lifetine regine is defined as the regine where

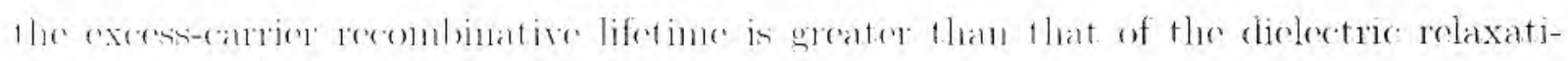

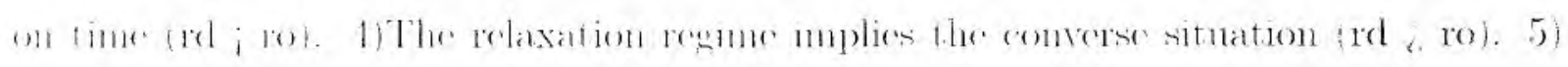

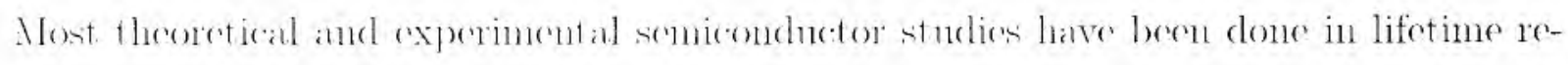
gine materials. 6) In contrast, relaxation semiconductors have received relatively little attention. 7) Many fundanental cuestions remain concerning nonecuilibrium transport in large-band-gap seniconductors. amorphoms and trap-elominated semiconductors. and

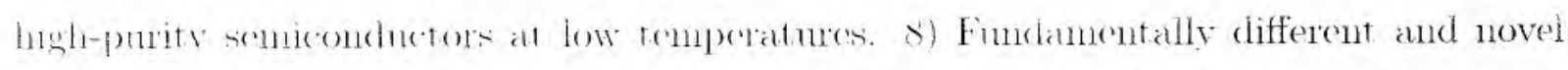
transport behavion can be observed in this high resistivity solich which are capable of

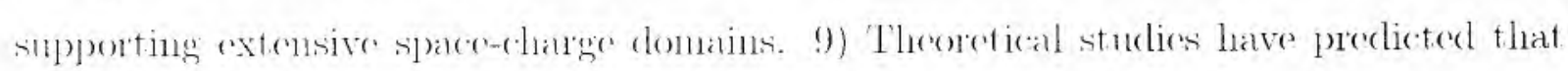
minority-andier injection into a relaxation semiconductor would result in majority-arrier depletion and the formation of sparededatege regions [2]. 10) Models solving the full set

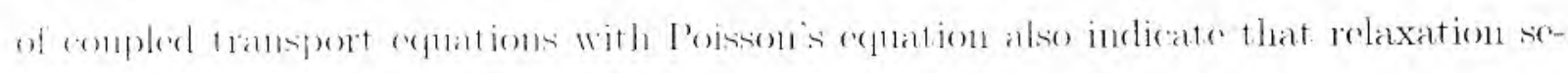

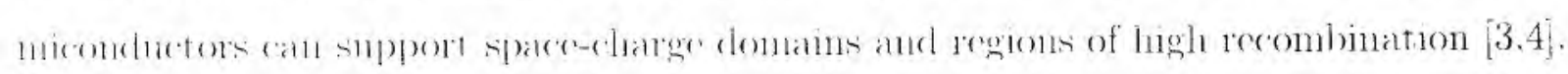
11) Essential predietions of redaxation therery inchude the formation of a space-charge dipole near an injecting contact and the existence of a recombination front where the excess carrier concentration (11p) profluct goes through a maximum. 12) The dipole is created by the combination of majority-aurier depletion near the injecting contact and a compesponding excess of majority carriers fouthere from injection which is recpured to 


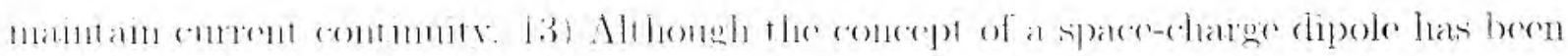

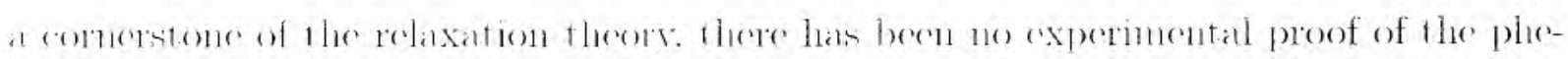
nomenom. 14) Previons experinental stmelies in redaxation semiconductors were limited by high deferet densitios which obsemerd much of the nuique physical phenomenta when

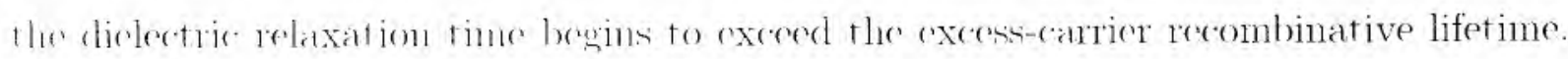
15) In this work. (aparitane measurements of a high-pmity silicon diode provide direct eridenes of a space-charge dipole in the relaxation regine phote reactive monolayer [3].

$$
\text { Questuen }
$$

1) Which sentener(s) or chanse(s) conkd be eliminated from the Introduction presented in the rearling frano withont losing critical information about the study?
a) 1 and 2
b) 1 (1) 4
(.) 5

6 - Language Conventions - Tense

Instrusan

Different ways to write the Stage IV of an introduction, together with four examples of Stages IV to papers in the field of Computer Science, is reproduced here. First read them, choose the best tense for each verb given in parentheses, then answer the questions.

\author{
E.tplicasaco \\ Orenetation and Telse
}

You may write the statenent of pupose (Stage IV) from one of two alternative orientations: I - The orientation of the statement of puppose nat be fowads the report itself. If you choose the report orientation you should use the present or finture tense. 


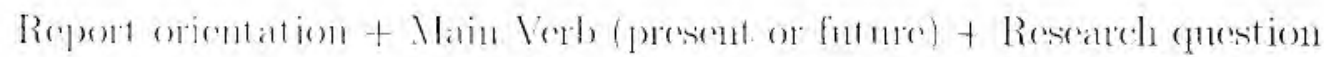

2- () the orientation of the statement of pmpose may be towards the researeh

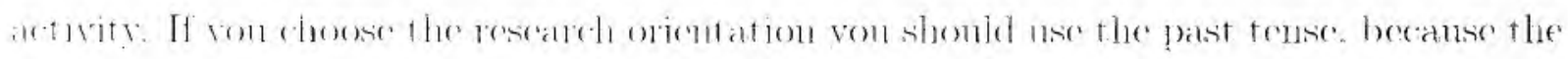
rescarde activity lats alleady beren completed.

Research orientation + Main Verb (past) + Rosearch (puestion

Trito

1. The thesis of this paper is that softwate enginerering advanees are recpured to

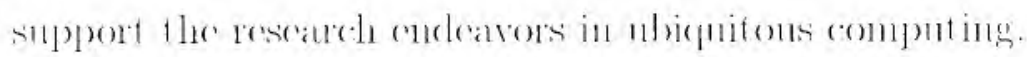

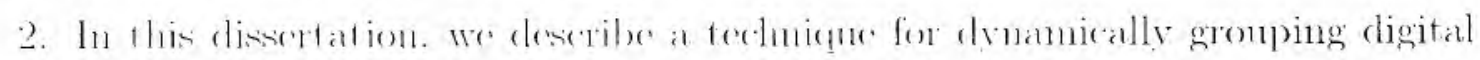

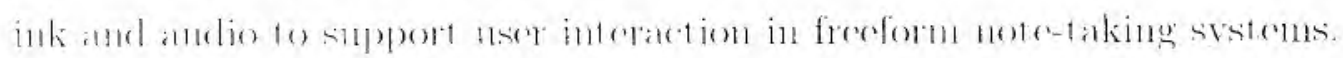

3. Thes report exammes the paretier of annotation in a particular sitnation: the markings students make in murersity-jesel texthooks.

4. The aim of the researeh reported here was to create tools (1) to support and (applure the froc-flowing activitios of mectings and (2) to utilize the captured multimedia meeting records effectively in the larger work processese in which the meetings are anterkiced.

5. In lesponse to this. Wo designed a study to andyatye people sinformation capture achivitics.

6. This paper presents a speede interface for a hand-hedel computer that allows

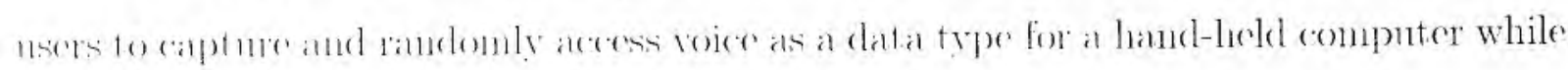

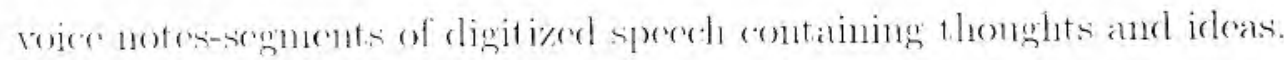

7. The purpose of the stucly reported in this paper is to begin to explore work in real settings in a systematie way. with a particular objective to inform the design of schision managenent models.

Questiors 
1) Which is the secpuenee that fill in the blanks of examples 1 to 3 ?
a) is drectilue. exannines
b) Wats. descrilsed. exilnitue
c) was deseriles. exammones

2) Which is the sepuenee that fill in the blanks of (xamples 4 to 6 ?

a) is. dosigned. present

b) Was designed. presents

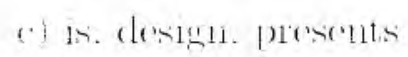




\section{Apêndice E}

\section{Exame formal realizado em Abril de}

\section{1}

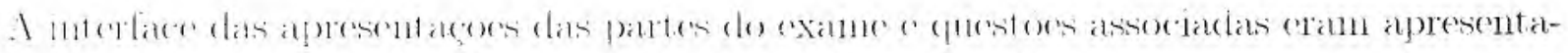

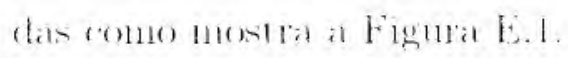

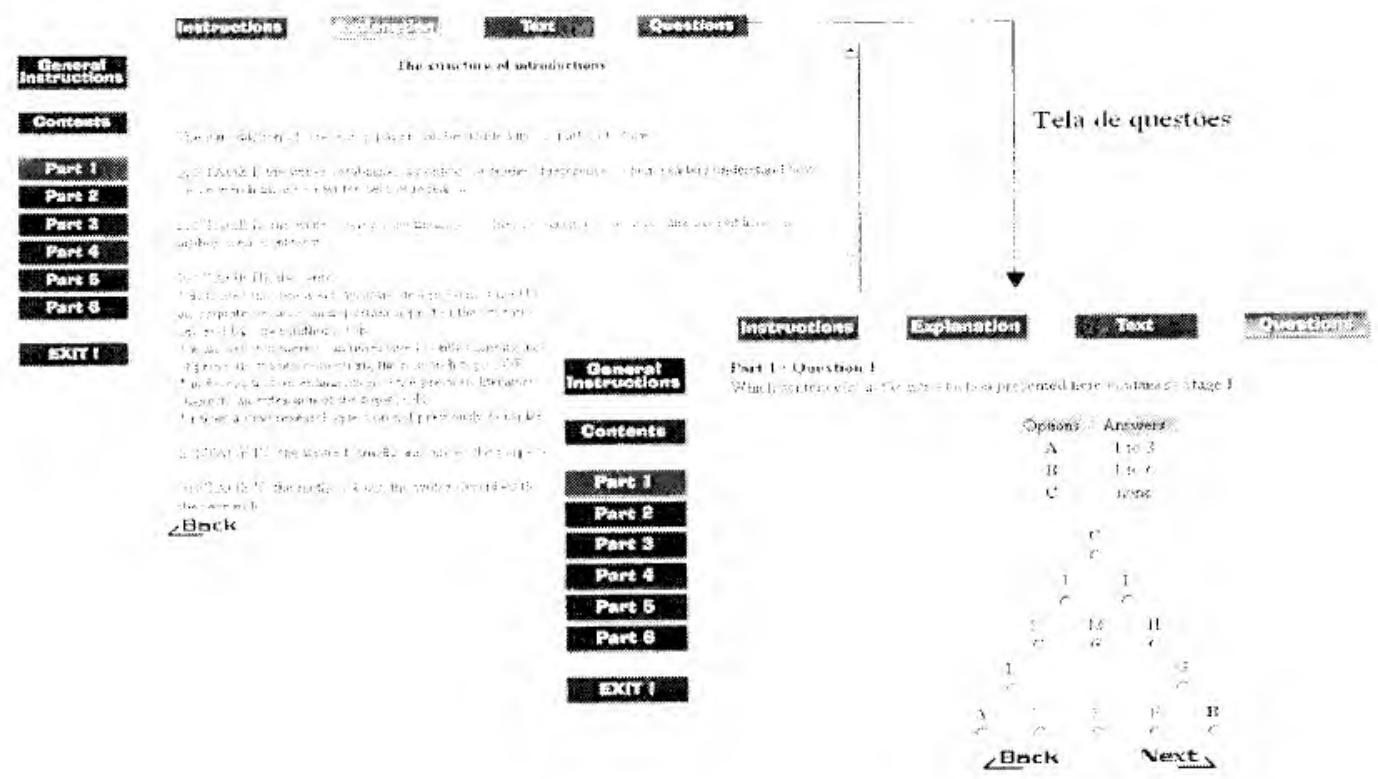

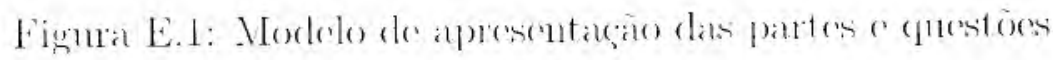

A sergnir săn apresentaulas exemplos das questón aplicadas para a área de Estatísticat. 


\section{1 - Divide and Classify the parts of an Introduction}

Insting(tic)

The description of the structure of introductions, together with an introduction to a paper in the field of Statistics, is reproduced here. First read them, then answer the questions.

\section{E.rplicuracios}

The introduction of a reseated paper (and be divided nuto s parts, or stages.

In STACiE I. the writer establishes a contexi. or frane of referenee to help readers mederstand low the rescared fits into a wirler field of researeh. In STAGE Il, the writer reviews the findings of oflere researchers who have alreaty published in his/her area of interest.

I1 STAGF. III:

* the writer indicates that previons literatume described in stage II is madequate becanse an inportant aspect of the rescanch area has been ignored by other anthors; or

* the writer indicates that there is an muresolved confliet annong anthors of previons studies concerning the research topic: or

* the writer indieates that an examination of the previons literature suggests an

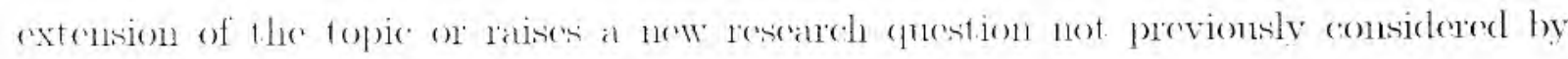

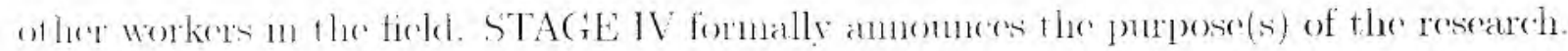

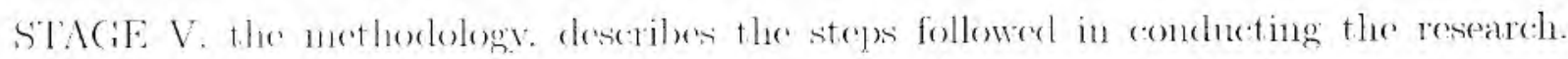
In STAGE VI. results. the writer presents the mann findings of the research. STAGE VII inclicates possible benefits or applieation of the rescareh. Sometimes. stage VII is combined with stage IV in the sime sentenee. Finally: in STAGE VIII, the writer presents the structure or layent of the paper. inclicating the sections and briefly commenting on them.

Some of the stages are optional and may depend on the jommal/conference style anel length of the article. Howeres. the general plan given in the reading frame is very 
(4) $111114(3) 1$

Trito

\section{How Many Clusters? Which Clustering Method?}

Anwers Via Model-Based Cluster Analysis

Technical Report No. 329

Department of Statistics

University of Washington

Introciuction

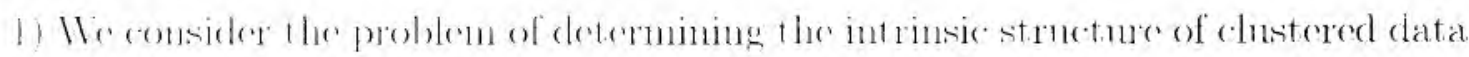

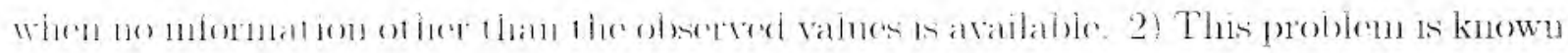

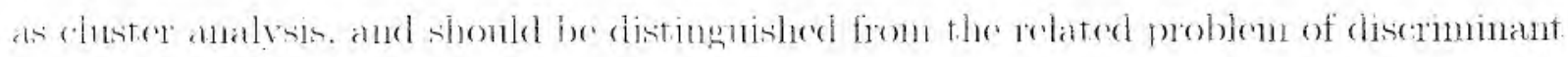
antalysis. in which known grempings of sonne observations are nesel to categorize others and infer the structure of the data as a whole. 3) Probability models have been proposed for (quite some time as a basis for (dusteremalysis. 4) In this approach. the clata are viewed as coming from a mixtme of probability distributions. each representing a different chuster. i) liecently. nestlests of this trpe have shown promise in a mumber of practical applica-

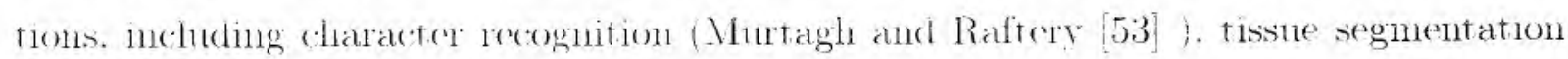
(Banfield and Raftery [7]). minefield and scismic fande detection (Dasgupta and Raftery [27]). iefontificatton of textile flaws from images (Camplodl of al. [21]). and clatsification

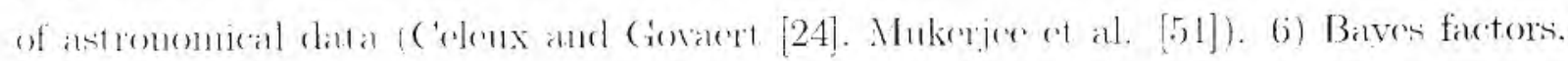
approxinated by the Bayesian Infomation Criterion (BJC). have bern applied successfulle to the problem of deternuming the munber of components in a model [27], [51] and for deciding which anomg two or more partitions most edosely matches the data for a

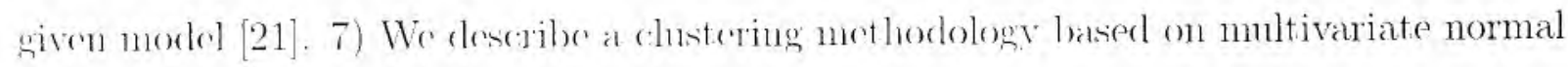
mixtmes in which the BIC is nosel for direct comparison of models that may differ not only in the number of components in the mixtnee. but also in the molerlying densities of the varions components. S) Partitions are determined (as in [27]) by a combination of hierarchical destreing and the EM (expectation-1naxinization) algorithm (Dempster. 
Land and Rubin [28]) for maximum likelihood. 9) This approach can give muth better performater than the rexisting mothexh. 10) Moreover. the E.M result also provides a measure of merertainty about the resilting chassification. 11) Figure 1 shows an example in which moded-hased elassification is able to mateh the clinieal elassification of a biomedical

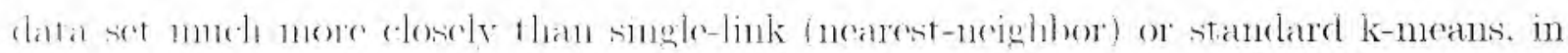
the absener of any traning data. 12) This paper is organized as follows. 13) In section 2 , we gire the necessary backgromed in multivariate chuster andysis. inchuding discissions of probability models. Ihe EM algorithu for chustering and approximate Bayes factors. 14) The basic model-based strategy and modifications for handling noise are described in Sertions 2.5 and 2.6. Iesperedivels: 15) A detailed analysis of the multivariate datia set

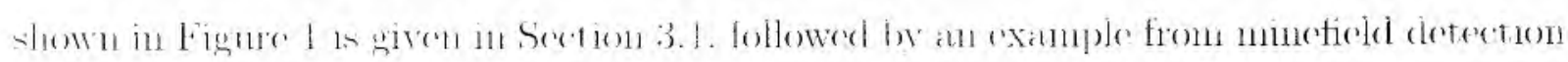

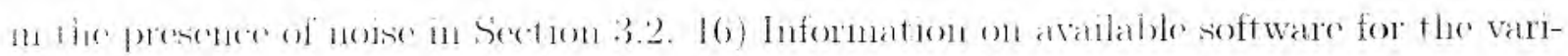
ons procedures nesel in this approand is given in Soction 1. 17) A fintal section smmmarizes and indicates exterisions to the med heol.

(Qurestions

1) Which sentenerfst in the introduction presented here containfsis Stage I?

a) 1

b) 1 and 2

(c) $1+05$

2) Which sentenceds) in the introduction presented here contain(s) Stage II?

(1) $2 i 01$

1) 3106

(.) 6

3) Which sentenee(s) in the intrednction presented here contain(s) Stage IV?
(1) 7
1) 7 and 8
c) 7 to 10 


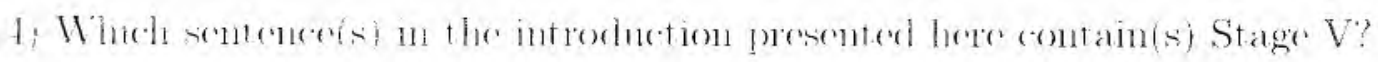

a) 8

b) 8 and ! 9

(.) 81010

2 - Classify and Rearrange the parts of an Introduction

Instruriolo

The following sentences in block were taken from an Introduction to a paper in the field of Statistics. The blocks are given here in scrambled ordering. The title of the paper is also given. First read them, then answer the questions.

Terte

Some Second Order Asymptotics in Exponential Family Non-Linear Regression Models (A Geometric Approach) Australian Journal of Statistics, $39(2), 1997,129-148$

1. Introduction

1. Efrom (1975) and Anari (1982a.1) introdues a differential geometric framework

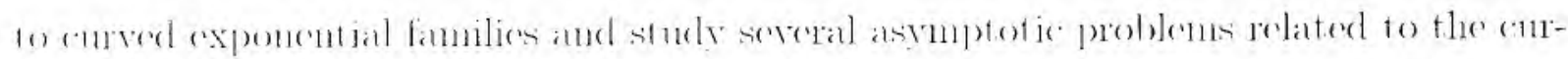

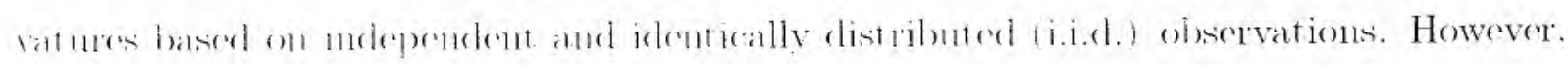

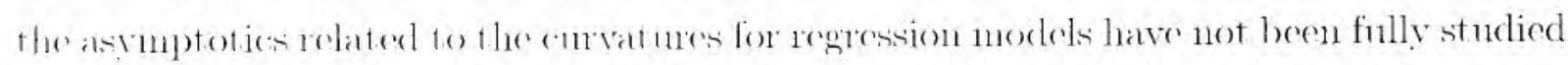
in the literature becantse there are some specific problems with regression models.

2. In this paper. we apply the geometric framework of Amari (1985) and tensor notation to stude some second order astmptotics for estimator in exponential family nondinear regression moders.

3. In recent vars. differential goometry has played an increasingly important role 


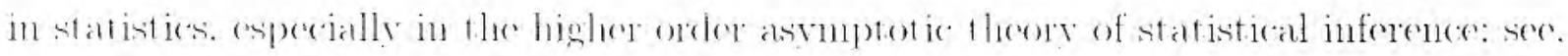

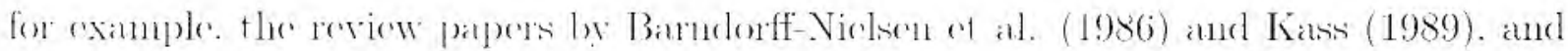
books be Anary (1985) and Murray of Rice (19993).

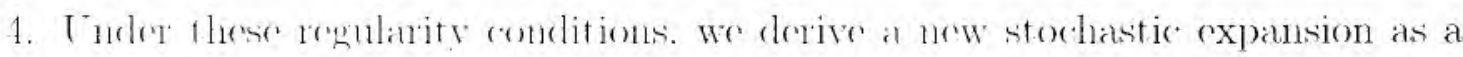
toof to study the asvmptotics for exponential fanily nonlinear regression models. Our result nav be regarded as further developments of previons work be Efron (1975). Efron (d Hinkley (1978). Amari (1982i,1) and Wei (1991).

5. In face there are two essential diflerences between curved exponential families with i.i.c. observations and regression modeds. First. the observations in regression models ane inderenelent but not neressarily identically distributed. Serond. the mainfold

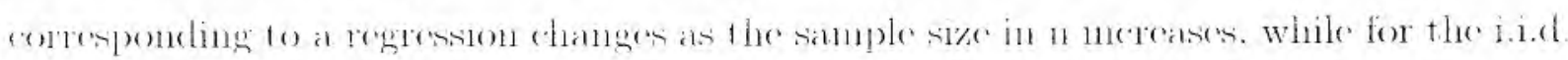

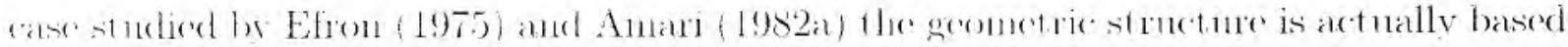
on one of the i.i.d. observations (Anari. 1985). Therefore the asymptotices in the i.i.d.

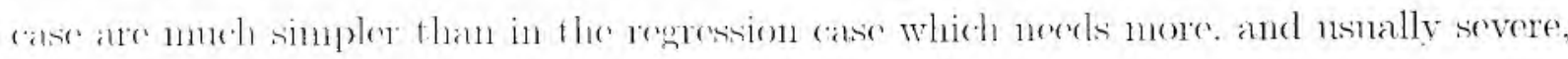
regularity conclitions.

6. Section 2 deseribes the gennetric asperts of exponential family nonlinear regresion modols based on the enometre framework of Amari (1985). Section 3 derives a new stochastic expansiom for first order efticient estimators. Section 4 investigates the information loss and gives a linit theorem comected with the observed information and the expected information. In Section 5. we calculate the hiats and the rovarianee of first

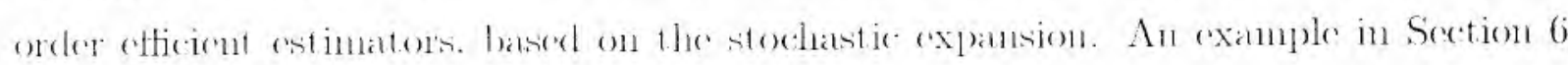
illustrates ont mentes.

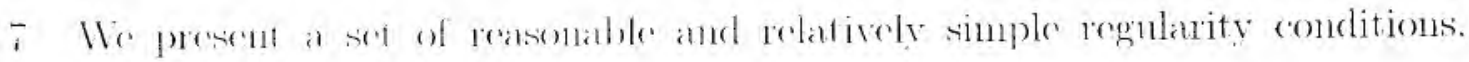
witle which to stucly astrmptotice from a geometric pont of vicw in regression models. (Our principal conditions are that gemetrie (puantitios such as the metrice curvature. comnertion and skewness tensor eorresponding to the regression model should exist on the arerage of observations.

\section{Questor's}

1) Which block in the introchetion presented leere is the most appropriated to 
al)
ii) 2
b) :)
(.) 5

2) Which block in the introduction presened lese is the most appropriated to apporar as the serenet one?
a) 1
b) 4
(.) 6

3) Which block in the introchution presented hare in the most appropriated to appear as the thirel ons?
i) 5
1) 4
(c) 7

4) Which hock in the introdnction presented here is the most appropriated to appear as the foruth one?
a) 6
1.) 7
(1) 2

5) Which block in the introduction presented here is the most appropriated to ap) (atr as the fifth onc?
i1) 2
(1) 1
(c) 7

3 - Strategies to write the Stages of an Introduction 
lirstruction

Different strategies to write the stages of an introduction, together with examples of Stages I of introductions to papers in the fields of Computer Science and Statistics, are reproduced here. First read them, then answer the questions.

Emplinacau

There are different alpproades to write the stages of an introduction. For example. the STAGE I an be written be wing there different strategies:

- argling abot't the topic prominenc'

- FAMIIATRI\%ING TERMS ()R ()B.JECTS OR PROCLSSES

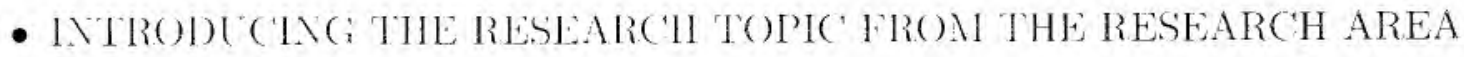

The first one nos arguments: the socond follows one of the three pattens: description. definition or classification-clivision: and the third follows the general to particular metering of terails

Irito

Eximulo 1:

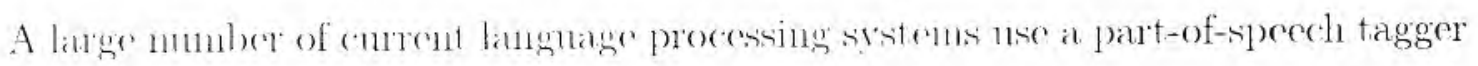
for pre-processing. The tagger assigns a (nuigur or ambignoms) part-of-speech tag to each token in the input and passes its ontput to the next processing level. nsually a parser. Furthermore, there is a large interest in part-of-speech tagging for corpus annotation projects. who create valuable linguistic resoneces by a combluation of antomatic processing 


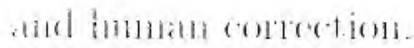

Example 2:

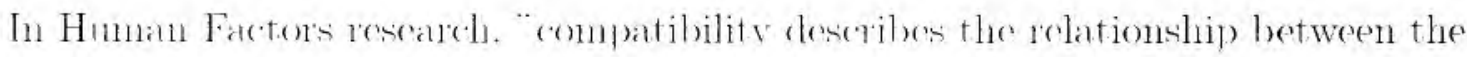
operation of an arteface and a persons expectations as to how that artefact should be

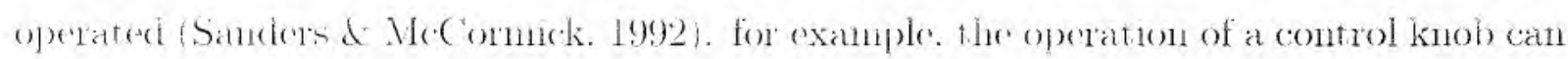
be influeneed by the "dockwise-to-increase" stereotype such that one expects a clockwise retation of the control knoh to leard to an increase in a controlled variable,e.g. volume on

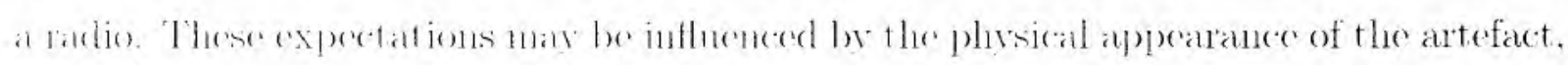

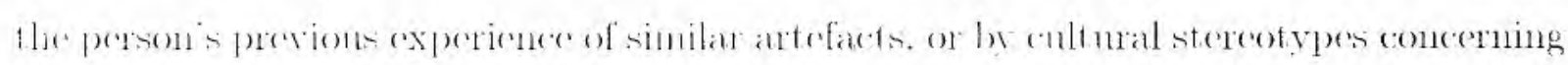

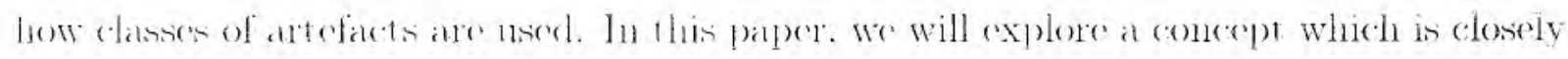

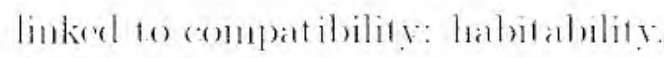

Examplo :3:

A financial market is a colloction of assets. which are dofned by their prices chan-

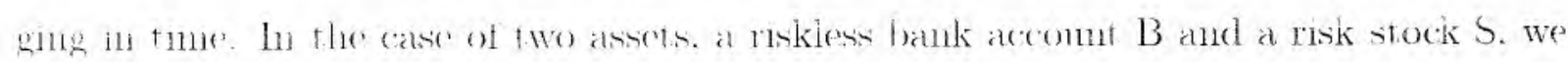
sperak of a (B. S)-market (sere [1]). A patticipant in such a manket contracting over some time interval (to buy or sell atsocts) adopts a contingent daim. which is defined by the whole prieresolution. The actions on the market redues to forming a portfolio (strategy)

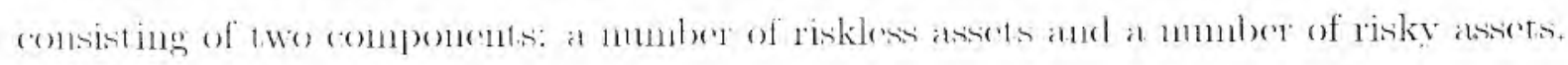

Questote

1) Which strategr follows the example 1?

a) Arguing alount the topic prominenee

b) Faniliarizing terms or objects or processones

(.) Introducing the rescench topice firom the rescarch area 
2) Which strategre follows 1 lie example 2 ?

a) Arguing about the topice prominemer

b) Fomiliarizing tertus or objects or processeses

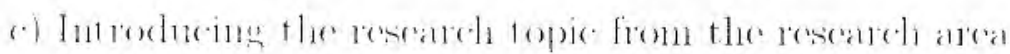

3) Which strategr follow the example 3"?

a) Arguing about the topic promincice

b) Finniliarizing termes or objects or processes

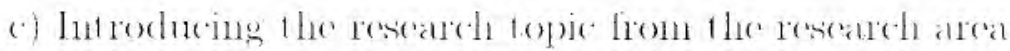

\section{4 - Summarization Deletion Operation}

Instrus,ain

The description of a summarisation operation, together with an introduction to a paper in the field of Computer Science, is reproduced here. First read them. then answer the questions.

\section{E.roplicacicion}

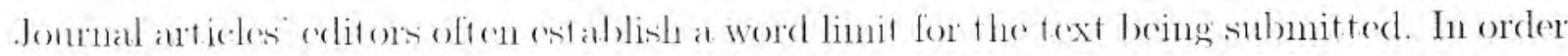

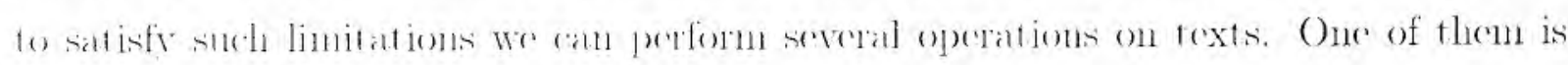

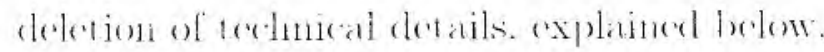

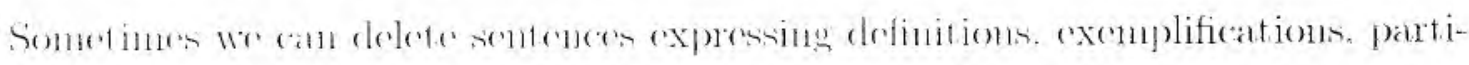

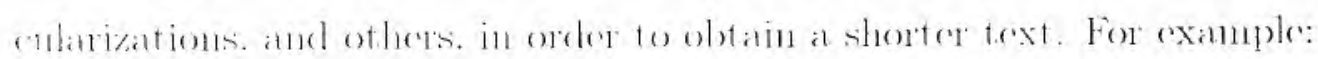

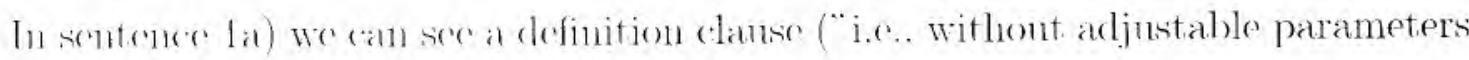
or empirical interaction potentials") that can be deleted to shorten the text. resulting the sentenese 11$)$.

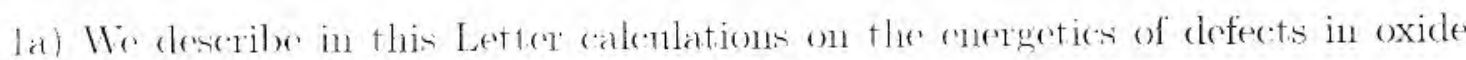

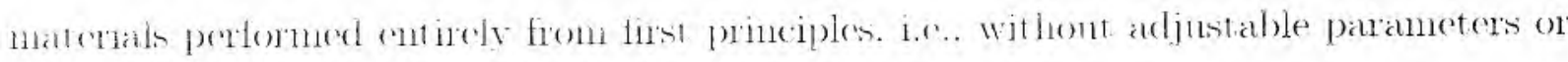
(moppirical interaction potentials. 


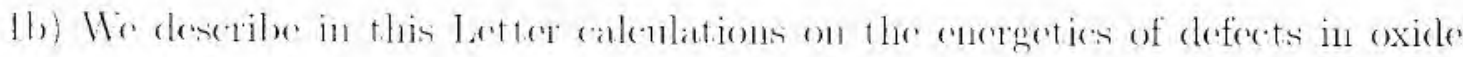
materials performed entirely from first prineiples.

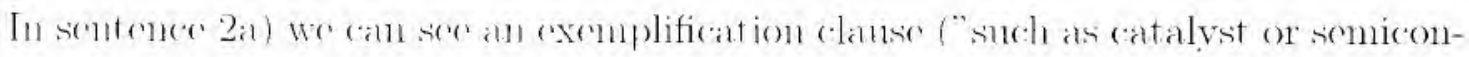

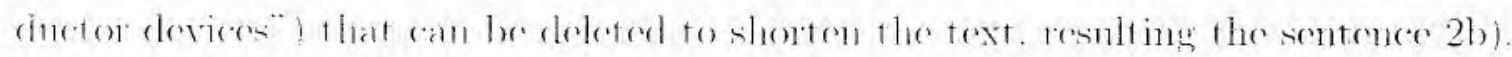

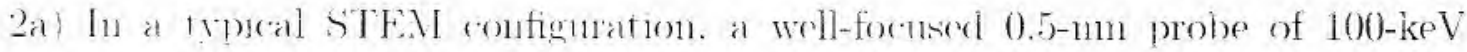
olectrons provides a high-pesolution transmission sauning indage for samples with complex

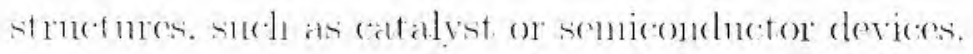

2b) In a trpical S'TEM configuation. a well-focosed (0.5-11m probe of $100-\mathrm{keV}$ electrons provides a high-1esolution transmission scanning indage for samples with complex -

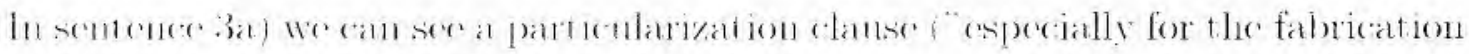

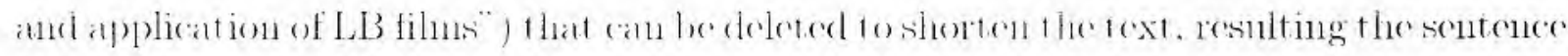
311).

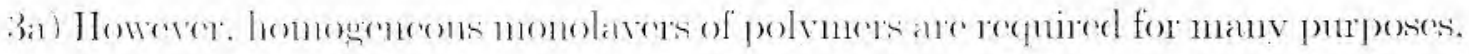
siperally for the fabrication and application of LB filus se techucpues for improving the lomogenelts of polyner momolavers are of moterest.

3h) However. homogeneons urousdavers of polvmers are repuired for many purposes. s) technignes for improving the homogeneity of polymer monolayers ale of interest.

Also. we (an apply the deletion operation when writing for knowleclgeable andienins.

Trate

\section{Effective View Navigation CHI 97 Electronic: Publications: Papers}

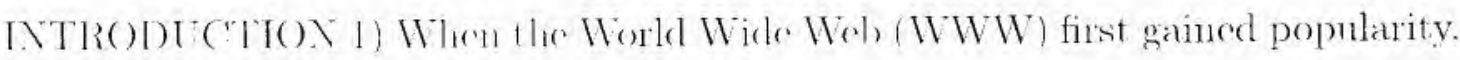
these who nsed it were impressed by the riehness of the content aceossible simply by wandering anound and (licking llings sern on the screen. 2) Soon after. struck by the near mupessibility of finding anctling specifice global navigation was largely abandoned in plate of search engines. 3) Whal went weong with pure navigation? 4) This work presented here serks theoretical insight into. in part. the problems with pure navigational aceess on the web, 5) More generally: it explores some basice issines in moring aromd 


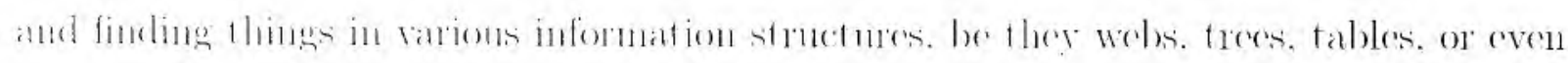

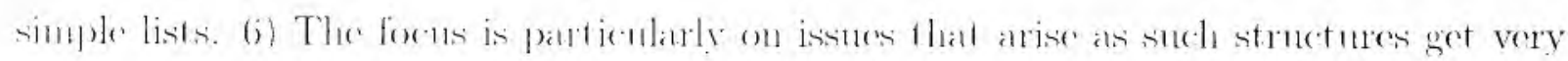

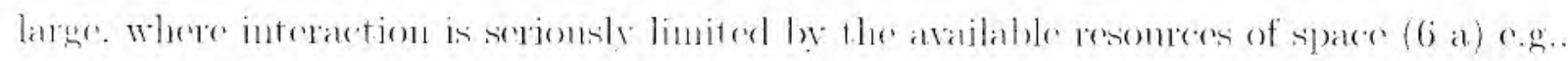

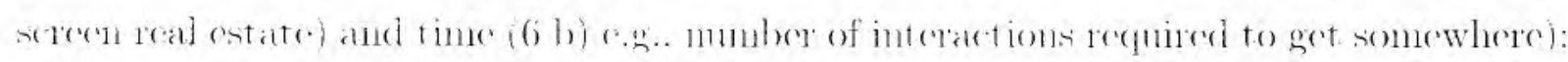

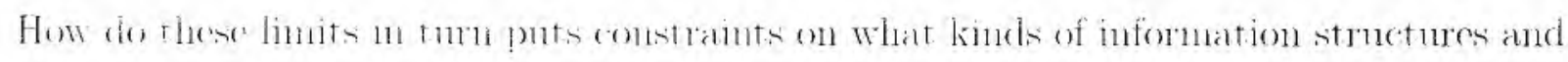
display strategies lead to effection navigation? 7) How lawe these constraints affected practice, and how might we live with them in future design? 8) We will be considering sustemo with statie structure over which users numst navigate to find things. e.g. lists.

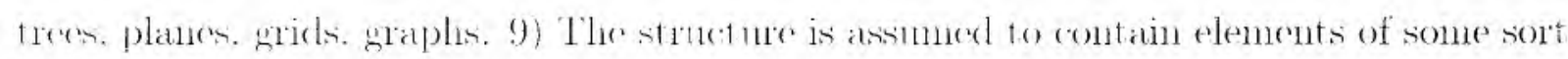

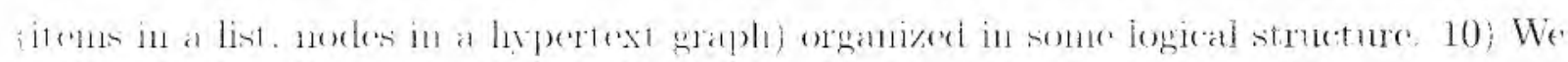

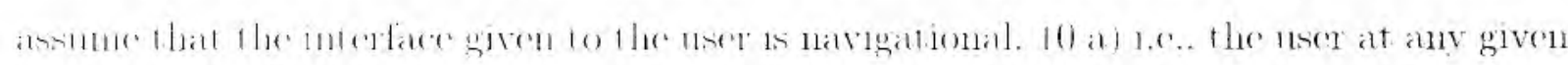

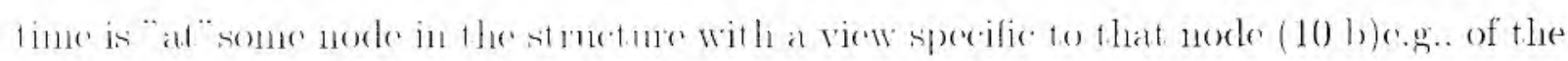
local neighborlood), and has the ability to move next to anything in that current view.

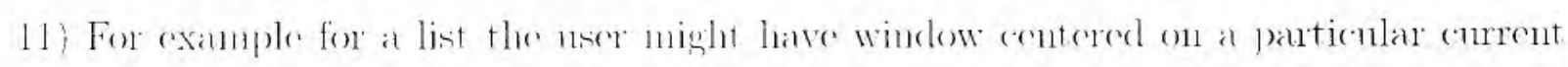

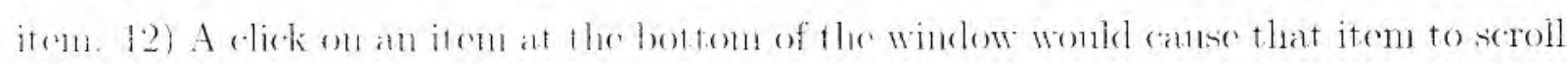

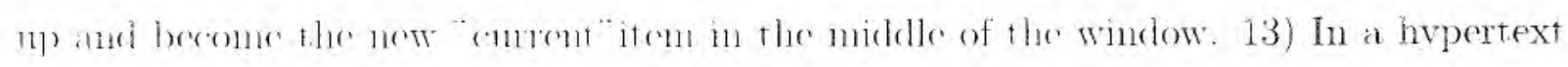
werb. a nore conld follow one of the visible links in the current hypertext page. 14) In this paper wo will first exantine viow traversal. the mule rlying iterative process of viewing. selecting something secen. and moring to it. to forme a path throngh the strueture. 15) Then we will leok at lhe nere complex riew navigat ion where in adedition the selections try

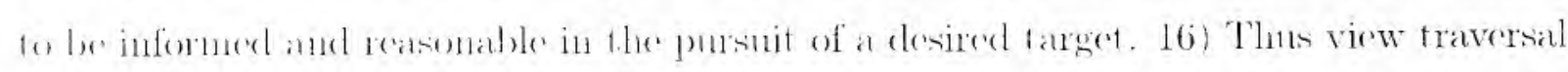

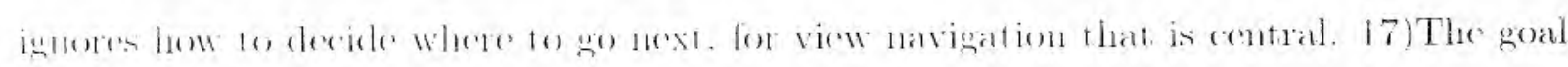

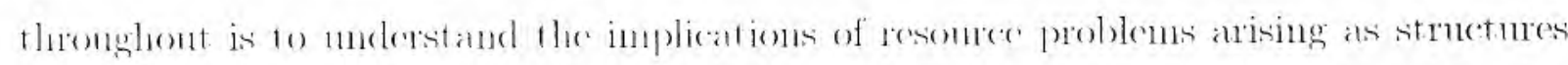
get very latges.

\section{Questors}

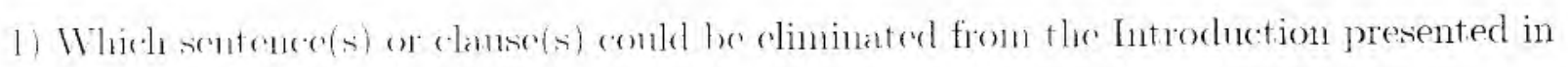
the reading frane without lowing critical information about the study?
a) 6 a. 6 b. 10 a. 10 b
b) 11
(.) 6 a. 6 b. 10 a. 10 b. 11 


\title{
5 - Language Conventions - Signal Words
}

Instimisio

Different ways to write the Stage III of an introduction, together with seven examples of Stages III to papers in the field of Computer Science, are reproduced here. First read them, then answer the questions.

\author{
Eirplicacus
}

Sighal Wirerks

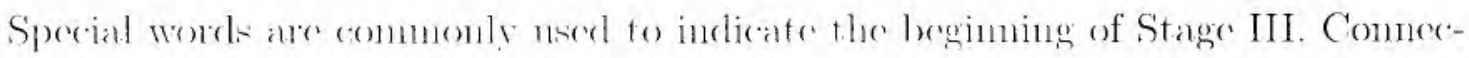

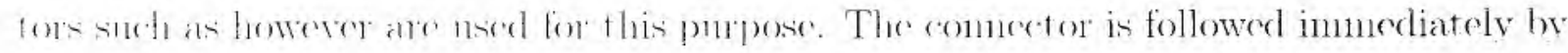
a gatp statement in the present or present perfort temse. Which often contans modifiers sucil an few lirtle. or no.

Signal word + Giap (present or present perfect) + Rescarch topic

Suborelinatheg entjunetions like while can also be used to signal Stage III. If you

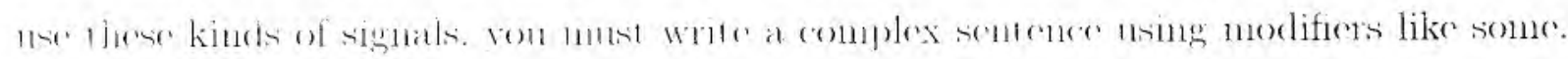

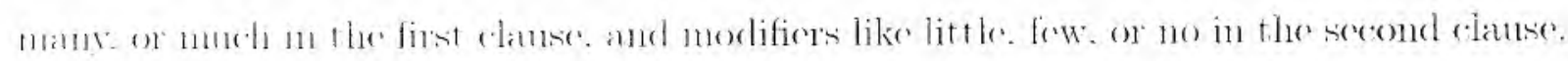

Signal word + Previons work (present or present perfect) + Gay) + topic

Texto

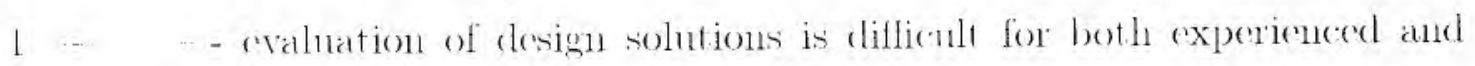
inexperienced designers. 


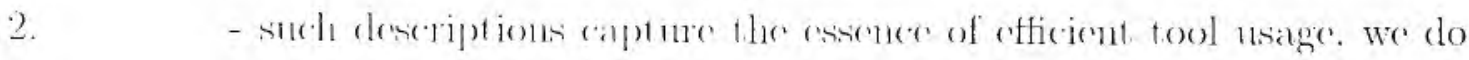
not ret understand hew to ged nsers to make efficient use of computer applications.

3. - the ker application area considered in this paper is that of enter-

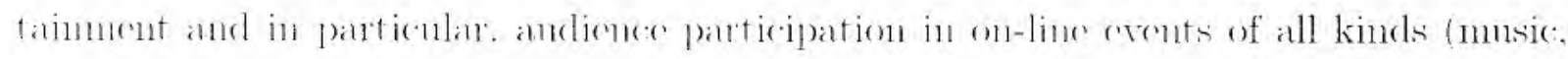

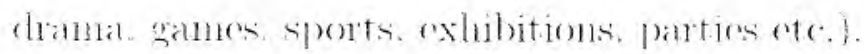

4. $\quad$ others have argued the benefits of learning progranming for its own sake $[6.9]$. om prinary interest is in progranming as a velucde to support conceptnal development in other areas.

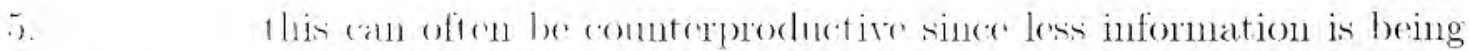
clisplaver. repuiring nore natigat ion.

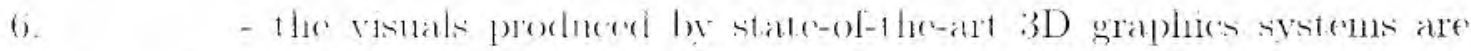

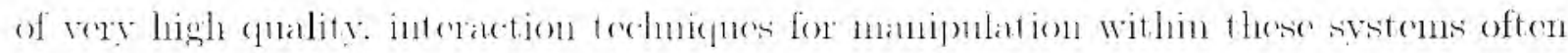

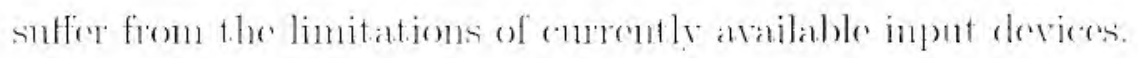

Questors 1)Which is the woth that fills in the hlank of the example 1 ?
a) Howeror
b) Whilo
1.) Althorigh

2) Which is the word that fills in the blank of the example 2?

a) But

1) While

(.) Howerey

3) Whied os the wored that fills in the blank of the example ?3?

a) Thongh

1) White

(.) Howerer

4) Which is the word that fills in the blank of the example 4?

a) Althongh

b) Bn11

(.) Howerल

$5)$ Which is the worel that fills in the blank of the example 5? 

a) Whilo
h) Theorght
(-) Howners
6) Which is the word that filk in the blank of the exannete 6 :
a) But
b) While
(c) Howerer
6 - Language Conventions - Tense
linsliue alu

Different ways to write the Stage IV of an introduction, together with four examples of Stages IV to papers in the field of Computer Science, are reproduced here. First read them. choose the best teuse for each verb given in parentheses, then answer the questions.

Explixaçio

Orientation and Tonse

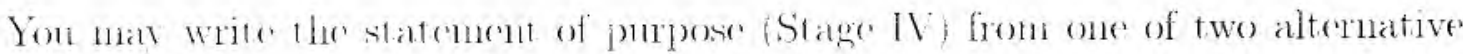
orentations: 1 - The orientation of the statement of purpose nat be towards the report

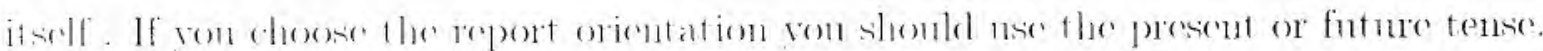

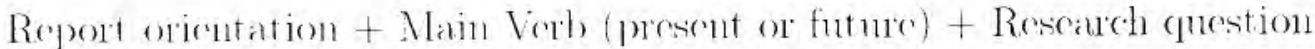

2- () the orientation of the statenent of pmonose may be towards the researeb activity. If you choose the researed orientation you shonld use the past tense. becanse the research activity has already beren eompleted. 
and finding things in varions information struetures. Io they wolds. trees, tables, or oven simple lists. (6) The forem is patt ienlarty on issues that arise as such structures get very

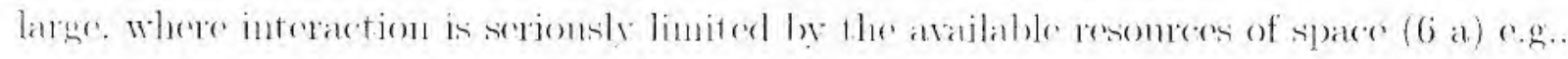

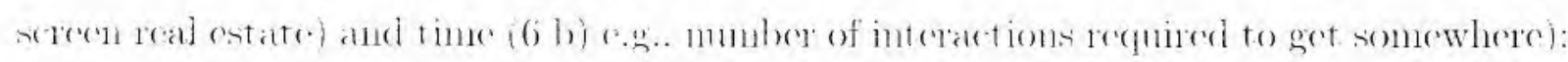
How do these linits 110 thm puts constrante on what kinds of information structures and display strategies lead to effective navigation? 7) How hase these constraints affected practice, and how might we live with them in future design? 8) We will be considering sustems with statie structure over which users must navigate to find things. e.g. lists. trees, planes griels. graphis. 9) The strueture is assmed to contain elements of some sort [iteme in a list. nextes in a hypertext graph) organized in some logical structure 10) We

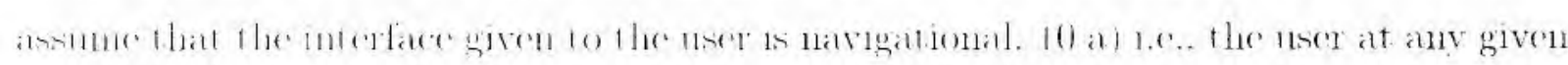

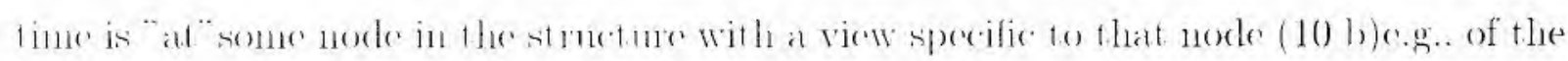

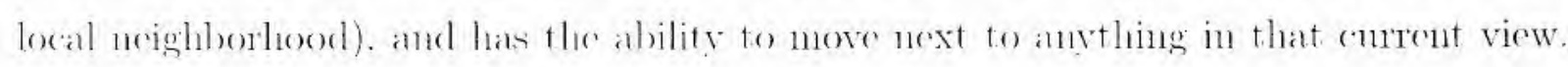
11) For exanuple for a list the noser night have window entered on a particular courrent

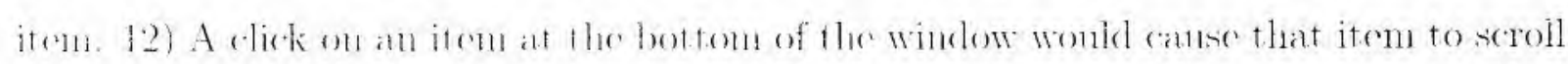
11] and berome the new " ancent" it ant in the midelle of the window. 13) In a hepertext werb. a nser conld follow one of the visible links in the current hypertext page. 14) In this papes wo will first exannine viow traversal. the melerlying iterative process of viewing. sclecting something secen. and moving to it. to forme a path thengh the structure. 15) Then we will look at the nere complex view navigat ion where in adedition the selections try

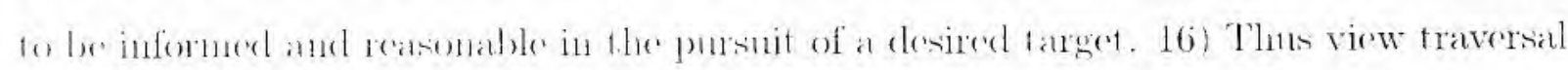

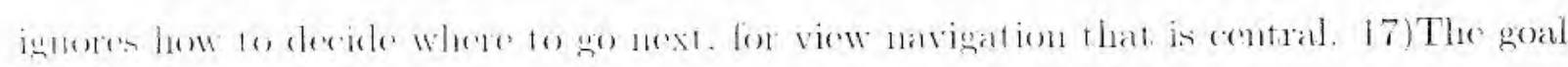

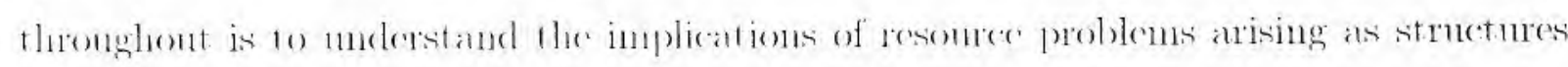
get very latges.

\section{Quistores}

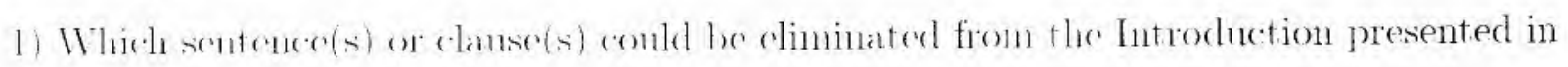
the reading frane withont losing eritical information about the study?
a) 6 a. 6 b. 10 a. 10 b
b) 11
(.) 6 a. $6 \mathrm{~b}$ b. 10 a. 10 b. 11 